Florida International University FIU Digital Commons

$11-5-2007$

\title{
Phylogenetic and Ecological Significance in the Evolution of Cetacean Tonal Sounds
}

Laura Johanna May-Collado

Florida International University, lmayc002@fiu.edu

DOI: $10.25148 /$ etd.FI08081532

Follow this and additional works at: https://digitalcommons.fiu.edu/etd

\section{Recommended Citation}

May-Collado, Laura Johanna, "Phylogenetic and Ecological Significance in the Evolution of Cetacean Tonal Sounds" (2007). FIU Electronic Theses and Dissertations. 44.

https://digitalcommons.fiu.edu/etd/44 


\section{FLORIDA INTERNATIONAL UNIVERSITY}

Miami, Florida

\section{PHYLOGENETIC AND ECOLOGICAL SIGNIFICANCE IN THE EVOLUTION OF CETACEAN TONAL SOUNDS}

A dissertation submitted in partial fulfillment of the

requirements for the degree of

DOCTOR OF PHILOSOPHY

in

BIOLOGY

by

Laura Johanna May-Collado

2007 
To: Interim Dean Mark Szuchman

College of Arts and Sciences

This dissertation, written by Laura Johanna May-Collado, and entitled Phylogenetic and Ecological Significance in the Evolution of Cetacean Tonal Sounds, having been approved in respect to style and intellectual content, is referred to you for judgment.

We have read this dissertation and recommend that it be approved.

Maureen A. Donnelly

Michael Heithaus

\begin{tabular}{cc}
\hline Tim Collins \\
\hline Zhenim Chen
\end{tabular}

Douglas Wartzok, Major Professor

Date of Defense: November 5, 2007

The dissertation of Laura Johanna May-Collado is approved

Interim Dean Mark Szuchman

College of Arts and Science

Dean George Walker

University Graduate School

Florida International University, 2007 
(C) Copyright 2007 by Laura Johanna May-Collado

All rights reserved. 


\section{DEDICATION}

To my husband thank for your endless support, guidance and love. To Douglas Wartzok and Maureen Donnelly for believing in me, thanks for giving me the opportunity to do what I like. A mi papá y mamá, los amo 


\section{ACKNOWLEDGMENTS}

This work was accomplished thanks to the support of many people and institutions. I would like to start with my committee members. Thanks to my mentor, Dr. Wartzok for accepting me as his student. The marine mammal field is a very competitive one, particularly for international students, but you gave an opportunity, and for that I will be always grateful. Thank you for being a wise and comprehensive supervisor, I just hope in the future I can be as good supervisor to my students as you have been to me. Thank you for your endless support and disposition. To 'Mo' Donnelly after many years of not seeing each other you still remembered me and believed in me enough to convince me to apply to FIU. Thanks for your power of convincing, guidance and support during all these years. Mike thanks for your enthusiasm, advice, and for reading my endless manuscripts. Thanks a lot for always have the time of to talk and reply my emails with. To Tim and Dr. Chen thank you for providing me with new tools in my career: phylogeny and statistics. Your classes inspired me and played a major role in this dissertation. Tim, thanks for always helping me and replying to my desperate emails after reviewers can back with revisions. Dr. Chen, you are the best stats professor. Thank you both of you for your advice, reading my work, and always replying to questions.

I am in debt to my dear friends, the 'Chicas Club' and the honoree member Brian Sidoti. To my lab mate Jennifer Lewis, thank you for making every day enjoyable no matter how hard things were. Your positivism is contagious friend. Thanks for reading my drafts, for fixing my internet connections, for picking up my mail, for providing me with food and water and many other things. Thank you Josette LaHee and Zayda Halun for making your visits to the lab so funny and refreshing. Thank you for providing me 
with food and a roof when I most needed it. Brian thanks so much for always being there your help and suggestions during our phylogeny class were of great help, we had a great time didn't we?

Thanks to my parents who ventured with me to the field to help me collect data and to take care of my little one, Amélie. Thanks to my parents in law who have always being there with their support. To Ingi Agnarsson, Monica Gamboa Poveda, Jose David Palacios, Diane, Chanya, Jorge May Barquero, Jose Damian Martinez, Jennifer Lewis, Sue and Doug Wartzok, and Evi Taubitz for helping in the field under tough sunny conditions. I would like to especially acknowledge Monica Gamboa and Jose David Palacios who became my field partners in Costa Rica, Panama and Ecuador, thanks for your support. To Sue Wartzok thank you very much for always lending me and my family a place to stay, thanks for your wonderful cooking, and for always being so great with us. I would like to thank all the staff at the Smithsonian Bocas del Toro Station, particularly Rachel Collins and Gabriel Jacome for always giving us a hand and support the project. I would like to thank greatly to Jon Coddingtong for making our family life possible. Thanks to Dr. Alvaro Morales and the section of Ecology from Universidad de Costa Rica, and Tia Janet for helping me to convince the Consul to give me a student visa. To my KETO fellows for creating an institution I can believe in. Finally, but not least to my dear husband, who became my star photographer, my advisor and my collaborator. Thank you for always being there for me. 
Funding for this dissertation came from many sources: Cetacean Behavior and Conservation Award by the Animal Behavior Society, Tinker Research Opportunities Award, LAAC-FIU, Lener-Gray Research Award by The American Natural History Museum, a Pre-doctoral Travel Fellowship by STRI-Bocas del Toro Marine Station, Judith Parker Travel Grant, Cetacean International Society, The Latin American Student Field Research Award by the American Society of Mammalogists, WWF-IIE-Russell E. Train Scholarship, and Dissertation Year Fellowship from Florida International University. I would like to thank particularly the Russell E. Train Fellowship, WWF, IIE, and Florida International University for giving me these fellowships that allowed me to dedicate full time to accomplish this dissertation. 


\title{
ABSTRACT OF THE DISSERTATION \\ PHYLOGENETIC AND ECOLOGICAL SIGNIFICANCE IN THE EVOLUTION OF CETACEAN TONAL SOUNDS
}

\author{
by \\ Laura Johanna May-Collado \\ Florida International University, 2007 \\ Miami, Florida \\ Professor Douglas Wartzok, Major Professor
}

Cetaceans are aquatic mammals that rely primarily on sound for most daily tasks. A compendium of sounds is emitted for orientation, prey detection, and predator avoidance, and to communicate. Communicative sounds are among the most studied Cetacean signals, particularly those referred to as tonal sounds. Because tonal sounds have been studied especially well in social dolphins, it has been assumed these sounds evolved as a social adaptation. However, whistles have been reported in 'solitary' species and have been secondarily lost three times in social lineages. Clearly, therefore, it is necessary to examine closely the association, if any, between whistles and sociality instead of merely assuming it. Several hypotheses have been proposed to explain the evolutionary history of Cetacean tonal sounds. The main goal of this dissertation is to cast light on the evolutionary history of tonal sounds by testing these hypotheses by combining comparative phylogenetic and field methods. This dissertation provides the first specieslevel phylogeny of Cetacea and phylogenetic tests of evolutionary hypotheses of cetacean communicative signals. Tonal sounds evolution is complex in that has likely been shaped by a combination of factors that may influence different aspects of their acoustical 
structure. At the inter-specific level, these results suggest that only tonal sound minimum frequency is constrained by body size. Group size also influences tonal sound minimum frequency. Species that live in large groups tend to produce higher frequency tonal sounds. The evolutionary history of tonal sounds and sociality may be intertwined, but in a complex manner rejecting simplistic views such as the hypothesis that tonal sounds evolved 'for' social communication in dolphins. Levels of social and tonal sound complexity nevertheless correlate indicating the importance of tonal sounds in social communication. At the intraspecific level, tonal sound variation in frequency and temporal parameters may be product of genetic isolation and local levels of underwater noise. This dissertation provides one of the first insights into the evolution of Cetacean tonal sounds in a phylogenetic context, and points out key species where future studies would be valuable to enrich our understanding of other factors also playing a role in tonal sound evolution. 


\section{TABLE OF CONTENTS}

\section{CHAPTER}

CHAPTER I: INTRODUCTION .1

CHAPTER II: CYTOCHROME B AND BAYESIAN INFERENCE OF WHALE PHYLOGENY

Abstract

CHAPTER III: THE FRESHWATER DOLPHIN INIA GEOFFRENSIS

GEOFFRENSIS PRODUCES HIGH FREQUENCY WHISTLES

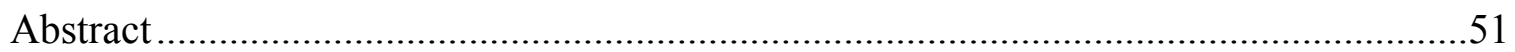

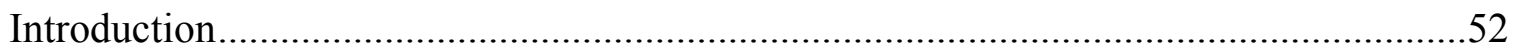

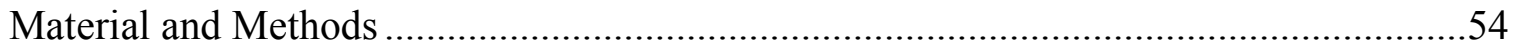

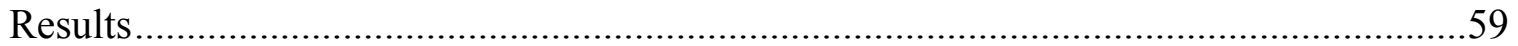

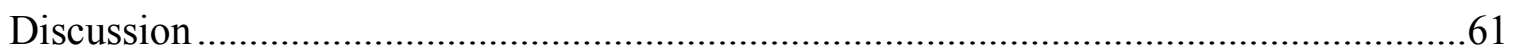

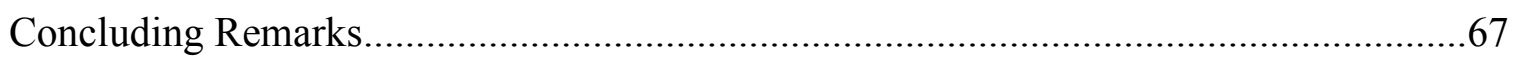

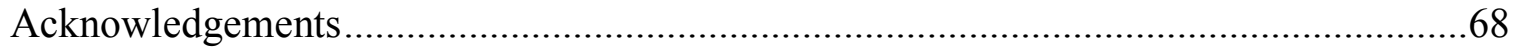

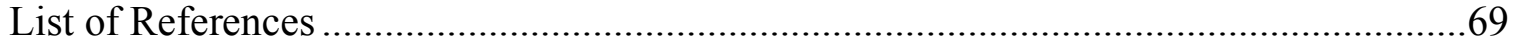

CHAPTER IV: REEXAMINING THE RELATIONSHIP BETWEEN BODY SIZE AND TONAL SIGNALS FREQUENCY IN WHALES: A COMPARATIVE APPROACH USING A NOVEL PHYLOGENY

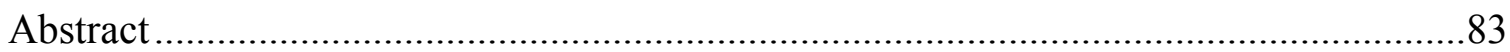

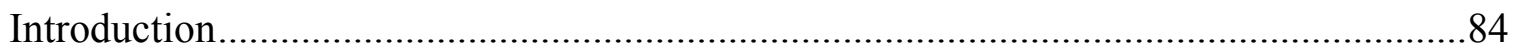

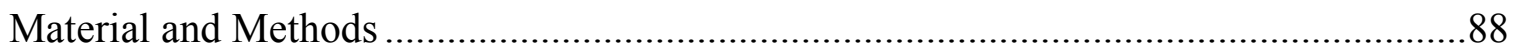

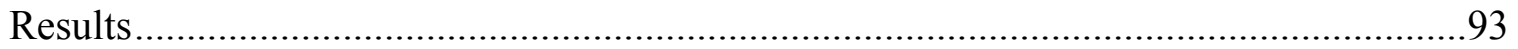

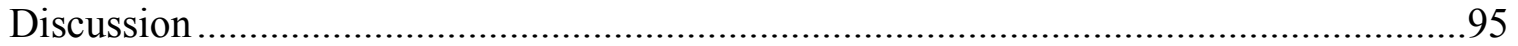

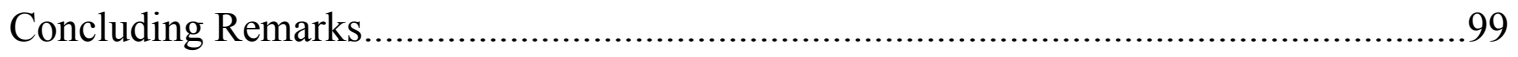

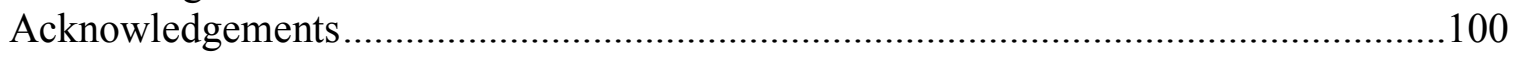

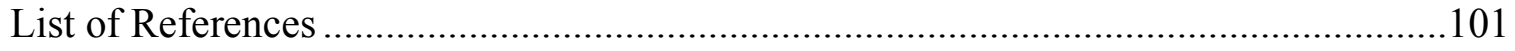




\section{CHAPTER V: PHYLOGENETIC REVIEW OF TONAL SOUND PRODUCTION IN WHALES IN RELATION TO SOCIALITY}

Abstract

CHAPTER VI: GEOGRAPHICAL WHISTLE VARIATION BETWEEN ADJACENT BOTTLENOSE DOLPHIN POPULATIONS

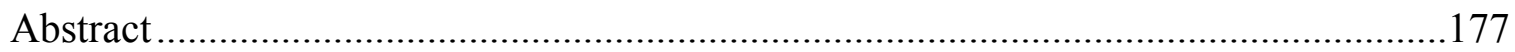

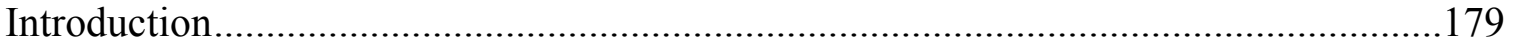

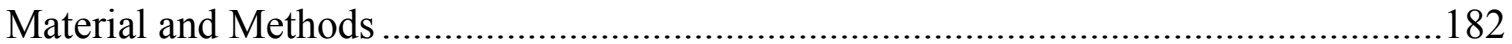

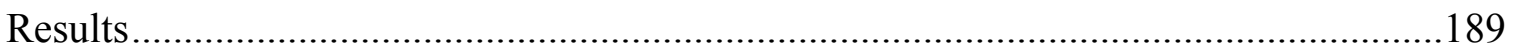

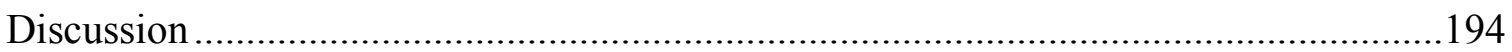

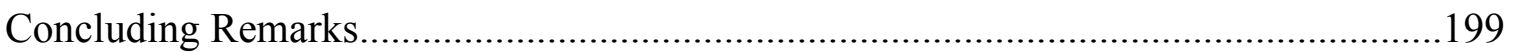

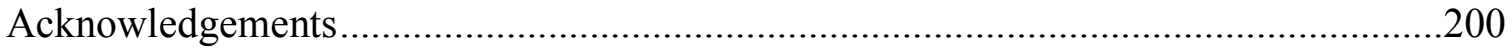

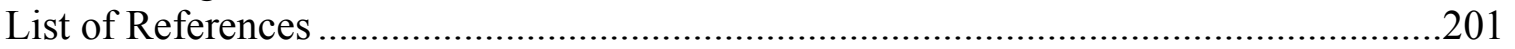

CHAPTER VII: CONCLUDING REMARKS .....................................................217

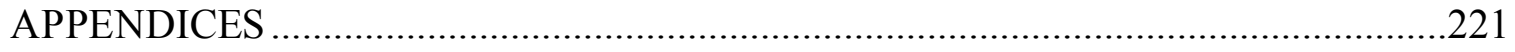

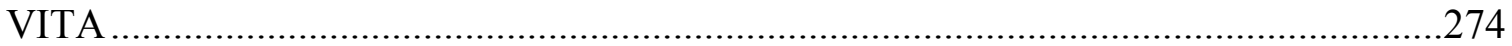




\section{LIST OF TABLES}

TABLE

PAGE

1. Species used in the analyses with respective GenBank accession numbers of cytochrome sequences.

2. Posterior probabilities and bootstrap values for all benchmark clade

3. Descriptive statistics of boto whistles with comparison to previously published data (in bold values for all 121 whistles and in parenthesis values for 70 whistles with frequency values below $25 \mathrm{kHz}$ for comparison purposes)

4. Descriptive statistics for each behavioral class for a total of 121 whistles recorded in relation to their behavioral context (there were not significant differences for any of these parameters across behaviors)

5. Whistle acoustic structure described for each recorded single animal and Group .

6. Review of published data on Cetacean body size and standard tonal sound frequency variables

7. Previous and present regression analyses between body size and standard tonal sound frequency variables. This study uses independent contrast both on the entire (values in parenthesis) and pruned (values in bold) data sets (see Methods)

8. Definitions of sociality and tonal sound characters and respective states

9. Probabilities of association between sociality (selecting the highest social state for polymorphic species) and tonal sound complexity. Significant positive associations at $\mathrm{p}$-values $>0.972$ and $0.973 * *$ for two and four state complexity characters, respectively and significant negative associations at p-values $<0.028$ and $0.027 *$ for two and four state complexity characters, respectively

10. Descriptive statistics of whistle acoustic parameters for both dolphin populations (see Fig. 16).

11. Bottlenose dolphin standard whistle parameters from this study and with other studied populations in the Atlantic 
12. Pairwise comparison of whistle standard parameters between populations, significant values a the $p$-value level of $p<0.05$ 


\section{LIST OF FIGURES}

FIGURE

PAGE

1. The preferred phylogenetic hypothesis based on the Bayesian analysis of cetacean species and 24 outgroups. Major groups are labeled according to recent literature, Cetacea is indicated by bold branches on the cladogram. For posterior probability support values, see Fig.2.

2. The preferred hypothesis showing posterior probabilities (numbers above branches) from the Bayesian analysis. Stars below branches indicate benchmark clades

3. Map showing the location of the Yasuni River and groups of botos (white dots) recorded during this study.

4. Examples of whistles ( $\mathrm{y}$ axis $=$ frequency in $\mathrm{kHz}, \mathrm{x}$ axis=time in seconds) recorded from Inia geoffrensis geoffrensis in the Yasuni River, Ecuador (a)-(f). Examples of whistles recorded from Sotalia fluviatilis in the Napo and Aguarico River are given for comparison purposes (h)-(i)

5. Distribution of whistle acoustic parameters as a function of dolphin species where $*$ and ${ }^{\circ}$ represent outside and far outside values of the $50 \%$ central tendency (Inia=121 whistles, Sotalia=33 whistles)

6. Plot of maximum versus minimum frequency of all recorded whistles for each species (Inia $=121$ and Sotalia $=33$ whistles)

7. The preferred phylogenetic hypothesis based on the Bayesian analysis of 64 cetaceans and 24 outgroups (gray branches). Numbers at nodes represent the posterior probabilities values

8. Optimization of overall body length (m) on natural log scale. Species that are known not to produce tonal sounds are denoted with '*' while '?' indicates poorly known species.

9. Regression analysis between Cetacean body size and tonal sound absolute minimum (a-b) and maximum (c-d) frequencies after correcting for phylogenetic relationships

10. Optimization of Cetacean tonal sounds standard frequency parameters (in natural $\log$ scale). 
11. Optimizations of tonal sounds (a) and whistles (b) versus sociality using the broad concept approach (see additional file 1). A brief glance at the black branches (indicating presence of tonal sounds/whistles and 'complex' sociality) on each side does not suggest detailed correspondence of acoustic structure with sociality. In other words whistles have a different phylogenetic distribution than does complex sociality etc, indicating that their co-evolutionary history (if any) may be more complicated than previously thought

12. Optimization of group size in Cetacea (using natural log). Dark purple and blue colored branches indicate small groups and demark most of the 'basal' whales. More brightly colored (green, yellow and red) indicate larger groups. The phylogeny suggests gradual increase in group size in the lineage leading to Delphinidae, with independent evolution of huge groups (red) in several lineages and some reversals to smaller groups (e.g. Cephalorynchus hectori).

13. Optimizations of social structure as a four state character (a) leaving polymorphic species as such, (b) lowest social state, (c) highest social state. All analyses were done using the highest social state optimizations (see Methods).

14. Optimization of Cetacean tonal sound standard acoustic parameters (using natural log). Dark colors (purple and blue) indicate low values, while brighter colors (green, yellow, red) indicate higher values

15. Regression analysis between independent contrasts of mean group size and mean number of inflection points. One conspicuous outlier (arrow) represents a contrast including the killer whale (Orcinus orca) which forms relatively small social groups but produces highly modulated whistles. It has been proposed that the killer whale uses whistles in a manner different from any other delphinid to indicate motivational state. That multiple factors are at work shaping tonal sounds in cetaceans may obscure and make difficult to discover true co-evolutinary histories of characters. Accordingly when $O$. orca is removed from the analysis the regression between the two characters becomes stronger

16. Map showing the location of Wildlife Refuge of Gandoca-Manzanillo and Bocas del Toro, dots represent ambient noise recording stations

17. Distribution of whistles acoustic parameters as a function of population where ${ }^{*}$ and ${ }^{\circ}$ represent outside and far outside values of the $50 \%$ central tendency

18. Relationship between coefficient of frequency of modulation (COFM) and duration (s). 
19. Distribution of whistles acoustic parameters as a function of population across the Atlantic.

20. Differences between mean whistle standard parameters between sympatric sympatric and non-sympatric bottlenose dolphins and Sotalia

21. Distribution of whistles acoustic parameters as a function of population and behavior where * and ${ }^{\circ}$ represent outside and far outside values of the $50 \%$ central tendency

22. Distribution of whistles acoustic parameters as a function of 'site fideliy' for Bocas dolphins where ${ }^{*}$ and ${ }^{\circ}$ represent outside and far outside values of the $50 \%$ central tendency.

23. Median ambient noise levels as a function of study site and recording station for each study site. 


\section{CHAPTER I}

\section{INTRODUCTION}

Mammals are among the most morphologically and ecologically diverse vertebrate groups (Perrin 1991). They have colonized greatly contrasting habitats and therefore evolved various ways to communicate and monitor these environments by using visual, olfactory, tactile, and acoustic senses. Most mammals to some extent use all these senses, but aquatic mammals like cetaceans rely predominantly on sound. This dependence on sound stems from light limitations in aquatic environments (Richardson et al.1995). Light attenuates rapidly with depth, limiting visual interactions between sender and receiver. Olfactory senses are also less developed in cetaceans than in land mammals, limiting their use for communication purposes (Wartzok and Ketten 1999). Sound, however, has the advantage of having low attenuation, allowing for effective communication and monitoring of aquatic environments over relatively long distances. For instance, baleen whales produce low frequency sounds $(<100 \mathrm{~Hz})$, with very small absorption losses; some whales are able to communicate over hundreds of kilometers (Richardson et $a l .1995)$.

The sophistication of acoustic communication reaches its pinnacle in cetaceans. These animals emit a compendium of communicative sounds that include broadband frequency modulated tonal sounds and narrowband frequency pulsed sounds (i.e., bursts, razors, barks, etc.). Tonal sounds are perhaps the most studied of these sounds, and are produced by both baleen whales (Mysticeti) and toothed whales (Odontoceti) — sister clades containing all extant whales. Baleen whales produce tonal sounds called 'moans' and 'tones' that have fundamental frequencies generally below $5 \mathrm{kHz}$ (Richardson $e t$ 
al.1995, Clark 1990). In contrast, in toothed whales these tonal sounds are called whistles and have frequencies that typically range from $5-20 \mathrm{kHz}$ (Richardson et al.1995). In some species fundamental frequencies can go up to $48 \mathrm{kHz}$ (May-Collado and Wartzok 2007). Although similar in acoustic structure across all whales, tonal sounds are possibly produced by two different mechanisms; the larynx in baleen whales and a complex air sac system in toothed whales (Cranford 2000, Cranford et al.1999).

Tonal sounds are produced in a variety of contexts. Baleen whales are believed to use these sounds for long distance communication (e.g., blue whales Sirovic et al.2004) and in sexual contexts (e.g., right whales Clark and Johnson 1984, humpback whales Tyack 1983, Tyack and Whitehead 1983). In toothed whales they are used for group cohesion, recruitment during feeding activities, and overall communication (e.g., Dreher and Evans 1964, Caldwell and Caldwell 1965, Herman and Tavolga 1980, Janik 2000, Herzing 2000, Acevedo-Gutierrez and Stienessen 2004). In 'true' dolphins (Delphinidae), signature tonal sounds (referred to as 'signature whistles' by Caldwell and Caldwell 1965) allow for mother-calf recognition and formation of male-alliances (e.g., Caldwell and Caldwell 1965; Caldwell et al.1990, Fripp et al.2005, Herzing 2000, Janik 2000, Tyack 1997, 2000, Watwood et al.2004).

Comparative studies have shown that some acoustic parameters of tonal sounds such as duration and modulation tend to vary within species (e.g., Barzúa-Durán and Au 2002, 2004, Morisaka et al.2005a, Wang et al.1995a) whereas frequency components typically vary across species (e.g., Matthews et al.1999, Oswald et al.2003, Rendell et al.1999, Steiner 1981, Wang et al.1995b). Several hypotheses have been proposed to explain this variability. Intraspecific variation has been proposed to be the result of local 
adaptation to ecological conditions or geographical isolation and genetic divergence between groups or populations (e.g., Azevedo and Van Sluys 2005, Barzúa-Durán 2004, Barzúa-Durán and Au 2004, Morisaka et al.2005b, Rossi-Santos and Podos 2006, Wang et al.1995b, Camargo et al.2007, Ansmann et al.2007). Interspecific variation in frequency components may be product of zoogeographic relationships (Steiner 1981), habitat (Wang et al.1995a), morphological constraints (Matthews et al.1999, Podos et al.2002, Wang et al.1995a,), phylogenetic relationships (e.g., Steiner 1981, Matthews et al.1999, Wang et al.1995a), and sociality (Podos et al.2002, Herman and Tavolga 1985).

Despite recent technological advances in cetacean bioacoustics, fundamental evolutionary questions remain unanswered. For example, in what context did tonal sounds evolve? What are the selective forces driving intra- and interspecies acoustic signal differentiation? Previous studies addressing such questions have been made with little or no reference to phylogenetic relationships and correlation analyses have relied upon standard statistics, which assume species as independent data points. Interspecific comparative studies, however face problems of non-independence. Failing to account for known phylogenetic dependencies among related species and failing to recognize that similarity in size or tonal sounds may be due to common ancestry artificially inflates the number of observations (and degrees of freedom) and correlations or regressions based on such observations are suspect. Therefore, the goal of my dissertation is to cast light on the evolutionary history of tonal sound signals by (1) establishing a species level phylogeny to test some of the hypotheses of tonal sound evolution at the inter-specific level using phylogenetic comparative methods, (2) confirming the emission of tonal 
sounds on the river dolphin Inia geoffrensis which due to its phylogenetic position is particularly important in this study, and (3) evaluating the role of the environment, sympatry between species, and other species-intrinsic factors on the whistle structure at the intra-specific level.

The dissertation consists of five chapters. Chapters II-V are formatted according to the journals in which they have been published and chapter VI to the format of the journal to which it will be submitted.

In order to test the above hypotheses it was necessary to have a well resolved Cetacean species phylogeny, which was not available at the time. Chapter II thus presents the first species-level cetacean phylogeny, which is based on the mitochondrial gene, Cytochrome-b (cyt-b) and utilizes Bayesian phylogenetic methods. This chapter discusses the utility of cyt-b and the importance of exhaustive taxon sampling (including outgroups) and Bayesian methods in Cetacean phylogenetics. Although based on only a single gene, the phylogeny is deemed reliable - and thus appropriate for hypothesis testing - based on its concordance with well established benchmark clades previously supported by morphological and mitochondrial and nuclear DNA (May-Collado and Agnarsson 2006).

Another important issue to be addressed before testing the above hypotheses was the long controversy of tonal sound ('whistle') production by river dolphins. The controversy stems from the assumption that whistles coevolved as a social adaptation within delphinids, and therefore sounds produced by river dolphins, regardless of the similarities in acoustic structure with whistles, were not considered as such, because river dolphins are largely solitary. Because of the phylogenetic position of Inia geoffrensis it 
was key to 'solve' the controversy in order to understand the evolutionary history of Cetacean tonal sounds. Chapter III describes high frequency tonal sounds (i.e. whistles) produced by Inia geoffrensis in Ecuadorian waters, and proposes the hypothesis that whistles in this river dolphin may be emitted under a social context different from that of dolphins (May-Collado and Wartzok 2007).

Having produced a species level phylogeny and addressed the issue of whistle production in river dolphins the stage is set to test hypotheses explaining tonal sound acoustic structure variation across species. One hypothesis is that body size constrains frequency in Cetacean tonal sounds. Body size is one of the most important morphological factors believed to influence animal signal frequency (Marquet and Taper 1998). The general idea is that body size and the size of sound producing organs correlate (Fletcher 1992) and the size of vocal tract places physiological constraints on signal production. In cetaceans body size has been suggested as a major factor influencing both the maximum and minimum frequency of tonal signals (e.g., Matthews et al.1999, Podos et al.2002, Wang et al.1995a). Chapter IV reexamines this hypothesis in a phylogenetic context. The findings corroborate the relationship between body size and minimum frequency but suggest that to explain the variation observed in other tonal sound frequency parameters, alternative hypotheses are required. This chapter emphasizes the importance of taking into account phylogenetic relations in comparative studies and considers the other potential factors playing a role in tonal sound variation i.e., sociality and the environment (May-Collado et al.2007a).

Chapter V considers the role of sociality as a potential factor affecting tonal sound acoustic variation across species by using several new comparative phylogenetic 
methods. It addresses problems with evolutionary analyses of broad concepts like 'whistles' or 'tonal sounds' rather than their component characters. This chapter finds new correlations between social structure and some tonal sound parameters. It proposes novel hypotheses about the role of social structure in tonal sounds and criticizes the oversimplistic hypothesis that whistles evolved 'for' communication in Delphinidae (MayCollado et al.2007b).

Chapter VI discusses several potential sources of whistle acoustic structure variation at the intra-specific level, including habitat acoustics (ambient noise levels and boat-dolphin interactions), sympatry with other dolphin species, isolation, and behavior in two adjacent bottlenose dolphin populations in the Caribbean of Costa Rica and Panama.

Finally, Chapter VII presents the conclusions and questions that emerge from this dissertation suggesting future directions of study that would continue to increase our knowledge on the evolution of Cetacean acoustic signals. 


\section{CITED LITERATURE}

Ansmann, I. C., Goold, J. C., Evans, P. G. H., Simmonds, M., \& G. K. Simon. 2007. Variation in the whistle characteristics of short-beaked common dolphins, Delphinus delphis, at two locations around the British Isles. Journal of the Marine Biological Association of the United Kingdom, 87, 19-26.

Acevedo-Guiterrez, A., and S.C. Stienessen. 2004. Bottlenose dolphins (Tursiops truncatus) increase number of whistles when feeding. Aquatic Mammals, 30, 357-362.

Azevedo, A.F., \& M. Van Sluys. 2005. Whistles of tucuxi dolphins (Sotalia fluviatilis) in Brazil: comparisons among populations. Journal of the Acoustical Society of America, $117,1456-1464$.

Barzúa-Durán, M. C., \& Au, W. W. L. 2002. Whistles of Hawaiian spinner dolphins. Journal of the Acoustical Society of America, 112, 3064-3072.

Barzúa-Durán, M. C. 2004. Differences in the whistle characteristics and repertoire of bottlenose and spinner dolphins. Anais da Academia Brasileira de Ciencias, 76, 386-392.

Caldwell, M.C., and Caldwell, D.K. 1965. Individual whistle contours in bottlenose dolphins (Tursiops truncatus). Nature (Lond.), 207, 434-435.

Caldwell, M. C., Caldwell, D. K., \& P. L.Tyack. 1990. Review of the signature-whistle hypothesis for the Atlantic bottlenose dolphin. In The bottlenose dolphin (eds. S. Leatherwood and R. R. Reeves), pp. 199-234. San Diego: Academic Press.

Camargo, F. S., Rollo, M. M. Jr., Glampaoli, V., \& C. Bellini. 2007. Whistle variability in South Atlantic spinner dolphins from the Fernando de Noronha Archipelago off Brazil. Journal of the Acoustical Society of America, 120, 4071-4079.

Clark. C.W. 1990. Acoustic behavior of mysticete whales. In Sensory abilities of cetaceans (eds. J. Thomas and R. A. Kastelein), pp. 571-583. New York: Plenum Press.

Clark, C.W., \& J. H. Johnson. 1984. The sounds of the bowhead whale, Balaena mysticetus, during the spring migrations of 1979 and 1980. Canadian Journal of Zoology ,62,1436-1441.

Cranford, W. 2000. In search of impulse sound sources in Odontocetes. In Hearing by Whales and Dolphins (eds. W.W.L. Au, A.N. Popper, and R.R. Fay), pp. 156-224. London: Springer Press.

Cranford W., Amundin M., \& K. S. Norris. 1999. Functional morphology and homology in the odontocete nasal complex: implications for sound generation. Journal of Morphology, 228, 223-285. 
Dreher, J.J., \& W.E. Evans. 1964. Cetacean communication. In (ed. W.N. Tavolga), pp. 373-399. Marine Bioacoustics. Oxford: Pergamon Press.

Fletcher, N. H. 1992. Acoustic Systems in Biology. Oxford University Press, Oxford, England. 352 pp.

Fripp D., Owen C., Quintana-Rizzo E., Shapiro A., Buckstaff K., Jankowski K., Wells R., \& P. Tyack. 2005. Bottlenose dolphin (Tursiops truncatus) calves appear to model their signature whistles on the signature whistles of community members. Animal Cognition, 8, 17-26.

Herman L. M. \& W. N. Tavolga. 1980. The communication systems of cetaceans. In Cetacean Behavior: Mechanisms and Functions (ed. Herman L. M.), pp. 149-209. New York: John Wiley Son Publisher.

Herzing, D. L. 2000. Acoustics and social behavior of wild dolphins: implications for a sound society. In Hearing by whales and dolphins (eds. W.W. L., Au Popper, A.N., and R.E. Fay), pp. 225-272. London: Springer Press.

Janik, V. M. 2000. Whistle matching in wild bottlenose dolphins (Tursiops truncatus). Science 289,1355-1357.

Marquet, P.O., \& M.L. Taper. 1998. On size and area: patterns of mammalian body size extreme across landmasses. Evolutionary Biology, 12,127-139.

May-Collado, L. J. \& D. Wartzok. 2007. The freshwater dolphin Inia geoffrensis geoffrensis produces high frequency whistles. Journal of the Acoustical Society of America, 121, 1203-1212.

May-Collado, L. J., Agnarsson, I., \& D.Wartzok. 2007a. Reexamining the relationship between body size and tonal signals frequency in whales: a comparative phylogenetic approach. Marine Mammal Science. 23, 524-552.

May-Collado, L. J., Agnarsson, I., \& D. Wartzok 2007b. Phylogenetic review of tonal sound production in whales in relation to sociality. BMC Evolutionary Biology. In press.

Matthews, J.N., Rendell, L.E., Gordon, J.C.D., \& D. W. MacDonald. 1999. A review of frequency and time parameters of cetacean tonal calls. Bioacoustics, 10, 47-71.21

Morisaka, T., Shinohara, M., Nakahara, F., \& T. Akamatsu. 2005a. Geographic variations in the whistles among three Indo-Pacific bottlenose dolphin Tursiops aduncus populations in Japan. Fisheries Science, 71, 568-576. 
Morisaka, T., Shinohara, M., Nakahara, F. \& T. Akamatsu. 2005b. Effects of ambient noise in the whistles of Indo-Pacific bottlenose dolphin Tursiops aduncus populations in Japan. Journal of Mammalogy, 86, 541-546.

Oswald J.N., Barlow J., \& T.F. Norris. 2003.Acoustic identification of nine delphinids species in the eastern tropical Pacific Ocean. Marine Mammal Science, 19, 20-37.

Perrin, W. 1991. Why are there so many kinds of whales and dolphins? Bioscience, 41, 460-461.

Podos, J., da Silva, V.M.F., \& M. R. Rossi-Santos. 2002. Vocalizations of Amazon river dolphins, Inia geoffrensis: insights into the evolutionary origins of delphinid whistles. Ethology,108, 601-612.

Rendell, L. E., Matthews, J. N., Gill, A., Gordon, J. C. D., \& D. W. MacDonald. 1999. Quantitative analysis of tonal calls from five odontocete species, examining interspecific and intraspecific variation. Journal of Zoology, 249, 403-410.

Richardson, W. J., Greene, C. R. Jr; Malme, C. I. \& D. H. Thomson. 1995. Marine Mammals and Noise. Academic Press. 576 p.

Rossi-Santos, M. R. \& J. Podos. 2006. Latitudinal variation in whistle structure of the estuarine dolphin Sotalia guianensis. Behaviour, 143, 347-364.

Sirovic, A., Hildebrand, J. A., Wiggins, S. M., McDonald, M. A., Moore, S. E., \& D. Thiele. 2004. Seasonality of blue and fin whales calls and the influence of sea ice in the Western Antarctic Peninsula. Deep-Sea Research II, 51, 2327-2344.

Steiner, W. W. 1981. Species-specific differences in pure tonal whistle vocalizations of five western North Atlantic dolphin species. Behavioral Ecology Sociobiology, 9, 241246.

Tyack, P. L. 1983. Differential response of humpback whales, Megaptera novaeangliae to playback of song or social sounds. Behavioral Ecology and Sociobiology, 13, 49-55.

Tyack, P.L. 2000. Functional aspects of cetacean communication. In Cetacean Societies: Field studies of dolphins and whales (eds. J. Mann, R. Connor, P. L., Tyack and H. Whitehead), pp. 270-307. Chicago: The University of Chicago Press.

Tyack, P. \& Whitehead, H. 1983. Male competition in large groups of wintering humpback whales. Behaviour 83:132-154.

Wartzok, D. \& D. Ketten. 1999. Marine Mammal Sensory Systems. In Biology of Marine Mammals (eds. Reynolds J. E. III and S. A. Rommel), pp. 117-175.Washington DC: Smithsonian Institution Press. 
Wang, D., Wursig, B., \& Evans, W. E. 1995a. Whistles of bottlenose dolphins: comparisons among populations. Aquatic Mammals, 21, 65-77.

Wang, D. W., B. \& W. Evans. 1995b. Comparisons of whistles among seven odontocetes species. In Kastelein R. A., Supin A. (eds). Marine Mammal Sensory Systems of Aquatic Mammals. New York: Plenum Press. Pp.213-221.

Watwood S.L., Tyack P.L., \& R.S. Wells. 2004. Whistle sharing in paired male bottlenose dolphins, Tursiops truncatus. Behavioral Ecology and Sociobiology, 55, 531543. 


\title{
CHAPTER II
}

\section{CYTOCHROME B AND BAYESIAN INFERENCE OF WHALE}

\section{PHYLOGENY}

\author{
LAURA MAY-COLLADO ${ }^{1,2}$, INGI AgNARSSON ${ }^{3}$ \\ ${ }^{1}$ Department of Biological Sciences, Florida International University, 11200 SW $8^{\text {th }}$ \\ Street, Miami, FL 33199, USA. \\ ${ }^{2}$ Escuela de Biologia, Univeridad de Costa Rica, Apto. 2060, San Jose, Costa Rica \\ ${ }^{3}$ The University of British Columbia, Departments of Zoology and Botany, 3529-6270 \\ University Blvd., Vancouver, B.C. V6T 1Z4, Canada.
}

May-Collado, L. J. and I. Agnarsson. 2006. Cytochrome b and Bayesian inference of whale phylogeny.

Molecular Phylogenetics and Evolution. 38: 344-354. 


\begin{abstract}
In the mid 1990's cytochrome b and other mitochondrial DNA data reinvigorated cetacean phylogenetics by proposing many novel and provocative hypotheses of cetacean relationships. These results sparked a revision and reanalysis of morphological datasets, and the collection of new nuclear DNA data from numerous loci. Some of the most controversial mitochondrial hypotheses have now become benchmark clades, corroborated with nuclear DNA and morphological data; others have been resolved in favor of more traditional views. That major conflicts in cetacean phylogeny are disappearing is encouraging. However, most recent papers aim specifically to resolve higher-level conflicts by adding characters, at the cost of densely sampling taxa to resolve lower-level relationships. No molecular study to date has included more than 33 cetaceans. More detailed molecular phylogenies will provide better tools for evolutionary studies. Until more genes are available for a high number of taxa, can we rely on readily available single gene mitochondrial data? Here we estimate the phylogeny of 66 cetacean taxa and 24 outgroups based on Cytb sequences. We judge the reliability of our phylogeny based on the recovery of several deep-level benchmark clades. A Bayesian phylogenetic analysis recovered all benchmark clades and for the first time supported Odontoceti monophyly based exclusively on analysis of a single mitochondrial gene. The results recover the monophyly of all but one family level taxa within Cetacea, and most recently proposed super- and subfamilies. In contrast, parsimony never recovered all benchmark clades and was sensitive to a priori weighting decisions. These results provide
\end{abstract}


the most detailed phylogeny of Cetacea to date and highlight the utility of both Bayesian methodology in general, and of Cytb in cetacean phylogenetics. They furthermore suggest that dense taxon sampling, like dense character sampling, can overcome problems in phylogenetic reconstruction.

Keywords: Balaneidae; Cetancodonta; Cetartiodactyla; Delphinidae; Delphinoidea; Euungulata, Iniidae; missing data; mitochondrial DNA; Monodentidae; Mysticeti; Odontoceti; monophyly; Perissodactyla; Phocenidae; Platanistidae; phylogeny; taxon sampling; Ziphiidae. 


\section{INTRODUCTION}

Several issues of Cetacean phylogenetics have been intensely debated, as a result of independent datasets (morphology, nuclear DNA, and mitochondrial DNA) suggesting conflicting hypotheses. These debates include the phylogenetic placement of Cetacea as sister to Artiodactyla (e.g., O'Leary and Geisler, 1999; Luckett and Hong, 1998; see also Gingerich et al., 1990) or embedded within Artiodactyla, a clade called Cetartiodactyla (e.g., Graur and Higgins, 1994; Gatesy et al., 1996, 1999; Gatesy 1997; Hasegawa and Adachi, 1996; Montgelard et al., 1997; Thewissen and Madar, 1999; Thewissen et al., 2001; Shimamura et al., 1999; Nikaido et al., 1999; Lum et al., 2000; Matthee et al., 2001; Arnason et al., 2004; Murphy et al., 2001; Reyes et al., 2004), the relationship between toothed whales and baleen whales (e.g., Luckett and Hong, 1998; Cerchio and Tucker, 1998; Douzery 1993, Messenger and McGuire, 1998; Nikaido et al., 2001; Nishida et al., 2003; Geisler and Sanders, 2003; Milinkovitch et al., 1993, 1994, 1995,1996; Milinkovitch, 1995, 1997), the relationships among delphinoids (e.g., Milinkovitch et al., 1993; Waddel et al., 2000; Nishida et al., 2003), dolphins (e.g., Mead, 1975; Kasuya, 1973; Barnes et al., 1985; deMuizon 1988; Perrin, 1989; Fordyce et al., 1994; LeDuc et al., 1999; Pichler et al., 2001), river dolphins (e.g., Flower, 1867; Winge, 1921; Slijper, 1936; Simpson, 1945; Cozzuol, 1985; Hamilton et al., 2001;

Cassens et al., 2000; Nikaido et al., 2001: Yan et al., 2005), and porpoises (Rosel et al., 1995).

Since the mid 1990's mitochondrial DNA data have been at the forefront of 
advancing understanding of cetacean phylogenetics (e.g., Arnason et al., 1992, 1993, 2004; Arnason and Gullberg, 1993, 1994, 1996; Milinkovitch et al., 1993, 1994; Irwin and Arnason 1994; Milinkovitch, 1995, 1997; Graur and Higgins, 1994; Gatesy et al., 1996, Montgelard et al., 1997; Sasaki et al., 2005), for several reasons. Mitochondrial DNA is relatively easy to amplify and sequence, it is mostly free of problems with paralogy, and it has a relatively high substitution rate and thus offers information at various phylogenetic levels (Irwin et al., 1991; Milinkovitch, 1997). Results based on mitochondrial DNA offered novel, often controversial hypotheses (e.g., Milinkovitch et al., 1993, 1994; Milinkovitch, 1995; Irwin and Arnason, 1994; Arnason and Gullberg, 1994) and sparked renewed interest in the reconstruction of the evolutionary history of whales. Some of these hypothesis such as the placement of Cetacea within Artiodactyla (Cetartiodactyla sensu Montgelard et al., 1997) (e.g., Graur and Higgins, 1994; Irwin and Arnason, 1994), and the unexpected hypothesis of the sister relationship of Cetacea and Hippopotamidae (Cetancodonta sensu Arnason et al., 2000) (see Irwin and Arnason, 1994; Gatesy 1997; Montgelard et al., 1997) have now received support from studies based on new independent datasets. Another unexpected mitochondrial hypothesis (based on Cytb, 12S, and 16S), the placement of baleen whales within toothed whales, however, was recently resolved in a different direction. Using the entire mitochondrial genome reversed the earlier mitochondrial hypothesis and recovered the monophyly of Odontoceti (Arnason et al., 2004). These previously controversial clades can now be labeled as 'benchmark' clades, i.e. to be likely true: 
Odontoceti: Arnason et al. (2004) (mitogenomic data); Messenger and McGuire (1998) (morphology); Nishida et al. (2003) (nuclear DNA); Nikaido et al. (2001) (retroposon SINE data); O'Leary et al. (2004) (combined morphology, nuclear DNA, mitochondrial DNA, and amino acids).

Cetartiodactyla: Thewissen et al. (2001), and Boisserie et al. (2005) (morphology including fossil taxa); Arnason et al. (2004) (mitogenomic data); Matthee et al. (2001) and Murphy et al. (2001) (nuclear and mitochondrial data); Shimamura et al. (1997, 1999) (retroposon SINE data); O'Leary et al. (2004) (combined morphology, nuclear DNA, mitochondrial DNA, and amino acids).

Cetancodonta (Cetacea + Hippopotamidae): Geisler and Sanders (2003) and Boisserie et al. (2005) (morphology including fossils); Gatesy et al. (1999) (nuclear and mitochondrial data); Lum et al. (2000) (retroposon SINE data); Arnason et al. (2000, 2004) (mitogenomic data); O’Leary et al. (2004) (combined morphology, nuclear DNA, mitochondrial DNA, and amino acids).

Some long standing debates are thus all but resolved: our understanding of deeper level cetacean phylogeny has grown strong. However, the strong focus of most recent studies, aiming specifically to resolve these higher level conflicts by adding mostly characters rather than taxa, has left our understanding of lower level relationships among whale species lagging behind. Mitogenomic data, for example, is available only for 16 cetacean species, and no molecular study to date has included more than 33 cetaceans. It seems timely to focus on more detailed (genus, and species level) molecular phylogenies. 
These will provide better tools for detailed evolutionary studies, and are necessary to test existing morphological phylogenetic hypotheses, and current cetacean classification.

Furthermore, adding taxa, as adding characters, can be an efficient way of overcoming phylogenetic uncertainty (Hillis, 1996, 1998; Graybeal, 1998; Pollock et al., 2002; Zwickl and Hillis, 2002; Hillis et al., 2003; but see Rosenberg and Kumar, 2001, 2003; Miller and Hormiga, 2004; Rokas and Caroll 2005). Obviously, combining multiple lines of evidence is beneficial to any phylogenetic problem. The study of O'Leary et al. (2004) is an excellent example of how seemingly incongruent data subsets can, when combined, yield a globally robust (and credible) result. However, until more genes are available for a high number of cetacean taxa, can we rely on readily available single gene mitochondrial data? Here we estimate the phylogeny of 66 cetaceans taxa representing 63 species, and 24 outgroups based on Cytb sequences from GenBank. This data matrix approximately doubles the taxon sampling of the most complete previous molecular study on cetacean phylogenetic relationships. We chose Cytb as it is available for more species than any other gene, and as it is a protein coding gene where alignment is trivial; in contrast many portions of the mitochondrial genome are notoriously difficult to align (e.g., Cerchio and Tucker, 1998; Messenger and MacGuire, 1998).

We judge the reliability of our phylogeny based on the recovery of the previously mentioned benchmark clades, in addition to the less controversial clades Perissodactyla, Euungulata (sensu Waddell et al. 2001, Perissodactyla + Cetartiodactyla), Cetacea, and Mysticeti. Because Cytb is thought to be most reliable at lower taxonomic levels (due to 
high substitution rates), recovering 'known' deeper clades gives credibility to these new findings which have not been addressed by studies using few taxa. We compare the performance of Bayesian analyses versus parsimony under four different models, and briefly examine the sensitivity of the results to taxon sampling. We use our results to discuss agreement and remaining conflict in cetacean phylogenetics, and provide comments on current classification. 


\section{MATERIALS AND METHODS}

Cytochrome data was compiled from GenBank for 66 cetaceans representing 63 species (see table 1 for accession numbers). Most previous mitochondrial DNA studies have included relatively few outgroups. For a stronger test of Cetartiodactyla monophyly and deeper level relationships we sampled 24 outgroup taxa using the recent mammalian phylogeny of Murphy et al. (2001) as a guide to outgroup choice. Murphy's et al. (2001) phylogeny, based on 18 gene segments, suggested the following relationships: (Carnivora (Perissodactyla + Cetartiodactyla)). Outgroups therefore include non-cetacean cetartiodactylans (16 species), Perissodactyla (six species), and two carnivores chosen as primary outgroups on which the preferred tree is rooted (Table 1). To minimize potential missing data problems in an already difficult phylogenetic problem, we chose to exclude cetacean taxa when the following two conditions applied: 1) only small partial Cytb sequences were available (less than $50 \%$ of the entire sequence), and 2) congeners with longer sequences were already present in the matrix.

The molecular matrices were matched and aligned using the Needleman-Wunsch algorithm (gap cost=10, mismatch=1) in MacClade 4.07 (Maddison and Maddison, 2003). As Cytb is a protein coding gene, the alignment of the Cytb sequences was unambiguous without any gaps.

The data were analyzed using Bayesian, and parsimony methods. The appropriate model for the Bayesian analyses was selected with Modeltest (Posada and Crandall, 1998), using the AIC criterion (Posada and Buckley, 2004) with a parsimony tree chosen 
as the basis for Modeltest. The best model was GTR $+\beta \S+\mathrm{I}$ (Rodríguez et al., 1990;

Yang et al., 1994). Estimates for the model parameters (-LnL=23900.7090, $\mathrm{K}=10$, base frequency $\mathrm{A}=0.368, \mathrm{C}=0.400, \mathrm{G}=0.0518, \mathrm{~T}=0.1802$ ).

Bayesian analysis was performed using MrBayes V3.0 (Huelsenbeck and Ronquist, 2001) with the following settings. The maximum likelihood model employed 6 substitution types ("nst=6"), with base frequencies estimated from the data. As substitution frequencies differ starkly between first, second and third positions in Cytb (Irwin et al., 1991), each codon position was treated separately (substitution rate partitioning) (charset 1st_pos $=1-1140 \backslash 3$; charset 2nd_pos $=2-1140 \backslash 3$; charset 3rd_pos =3-1140\3; partition bycodon = 3: 1st_pos; 2nd_pos; 3rd_pos; set partition = bycodon). Rate variation across sites was modeled using a gamma distribution (rates="invgamma"). The Markov chain Monte Carlo search was run with 4 chains for 5,000,000 generations(repeated three times), sampling the Markov chain every 1000 generations, and the sample points of the first 70,000 generations were discarded as "burn-in", after which the chain reached stationarity.

Parsimony analyses were done in PAUP* (Swofford, 1999) and NONA (Goloboff, 1993) through the WINCLADA shell (Nixon, 2002). In each of the analyses, heuristic searches were done with 1000 random stepwise additions, and subtree-pruning and regrafting branch swapping algorithm (chosen arbitrarily). As transitions (Ti) are much more common than transversions (Tv) and different codon positions show different levels of Ti saturations (third position showing the highest), we used some of the many 
weightings schemes suggested by previous authors. In addition to equal weights $(\mathrm{Tv}=\mathrm{Ti}$ $=$ all positions $=1)$, down weighting transitions $(\mathrm{Ti}=0, \mathrm{Tv}=1$, as suggested by Milinkovitch et al., 1996), ( $\mathrm{Ti}=1, \mathrm{Tv}=3$ as suggested by Milinkovitch, 1994, see Messenger and McGuire, 1998), unequal codon weighting (4:17:1 as suggested by Arnason and Gullberg, 1994).

Node support for the parsimony analyses was estimated using Bootstrapping (Felsenstein, 1985). Each analysis was run for 200 Bootstrap replicates, with 10 random addition sequences, and holding a maximum of 100 trees, per replicate.

To examine the effect of sparse taxon sampling on the Bayesian analysis (numerous previous studies have analyzed smaller Cytb datasets using parsimony) we analyzed two, rather arbitrarily chosen subset of the data. First, we pruned the dataset to contain a comparable taxon sampling to that of Messenger and McGuire (1998) subsample in Table 1; second, we used the pruned ingroup dataset, but added all the outgroups from the main data matrix (subsample, plus outgroups in Table). 


\section{RESULTS}

\section{Bayesian analysis}

The Bayesian analysis recovered all seven benchmark clades (Table 2). Support for five of the benchmark clades is high (100 posterior probabilities) but rather low for Cetancodonta (79) and marginal for the monophyly of Odontoceti (67) (Figure 1, Table 2). The analysis also recovered all but one family level, and most sub- and super-family level cetacean taxa (Figure 1, for posterior probability values for each clade, see Figure 2). The results thus broadly corroborate current cetacean classification, while also pointing to some lower-level groups that may need redefinition.

\section{Pruned Bayesian analyses}

The Bayesian analysis of pruned matrix I (see Table 1) was broadly congruent with the parsimony analysis of Messenger and McGuire (1998) based on a similar taxon sampling, rejecting Odontoceti monophyly. When all outgroups of the main matrix were added (subsample matrix II, see Table 1), however all the benchmark clades were again recovered (Table 2).

\section{Parsimony analyses}

The parsimony analyses all recovered Perissodactyla, Cetancodonta, Cetacea, and Mysticeti, with variable support (Table 2). Euungulata was recovered with high support by three out of the four analyses, but not under the $4: 17: 1$ weighting scheme. None of the 
parsimony analyses unambiguously recovered Cetartiodactyla or Odontoceti. Under equal weights, the majority of the most parsimonous trees supported Odontoceti monophyly while the strict consensus collapses Mysticeti, Kogia, Physeter, and Ziphidae+Platanista, into a pentachotomy with the remaining cetaceans. Cetartiodactyla is not recovered under equal weights, due to the placement of Camelus+Lama basal to Perissodactyla. Ignoring transitions altogether (see Milinkovitch et al., 1996) was similar to the equal weights analysis, although the strict consensus is less resolved, with the same pentachotomy formed at the base of Cetacea. Weighting transversions three times transitions (see Milinkovitch et al., 1994; McGuire and Messenger, 1998) placed Mysticeti sister to Ziphidae, in turn sister to sperm whales (Table 2).

Apart from benchmark clades, most analyses broadly agreed on the monophyly of superfamily, family, and subfamily level taxa within Cetacea. All cetacean families are supported with the exception of Balaenopteridae which consistently contained Eschrichtiidae. Subfamilies within Phocoenidae and Ziphidae were furthermore contradicted by all analyses. Our results support the transfer of Lagenorynchus acutus to Leucopleurus (as suggested by LeDuc et al., 1999, 2002), and in our preferred phylogeny (Figures 1-2) this taxon is place sister to Delphinae plus Stenoninae. The following genera are not monophyletic according to our results: Lagenorynchus (even after excluding L. acutus), Stenella, Phocoena, and Balaenoptera. 


\section{DISCUSSION}

\section{Recovery of benchmark clades}

Many recent cetacean phylogenetic studies include relatively few taxa (exceptions include Arnason and Gullberg, 1996; Messenger and McGuire, 1998; LeDuc, 1999, 2002; Hamilton et al., 2001), in part due to a focus on generating more characters to resolve higher level phylogenetics (see e.g., Lum et al., 2000; Nikaido et al., 2001; Nishida et al., 2003; Arnason et al., 2004). While addressing crucial questions and providing the backbone for lower level phylogenies, such studies have limited utility for classification, and for comparative evolutionary studies. In some cases sparse taxon sampling may also confound the results (Hillis et al., 2003). Of course, taxon sampling is usually simply constrained by the availability of character data, but for some reason many studies have opted to include only one, or a few outgroup taxa, even if many are available. Outgroup choice may have marked impact on any phylogenetic analysis (see e.g., Adachi and Hasegawa, 1995; Milinkovitch and Lyons-Weiler, 1997).

Here we have extensively sampled cetacean taxa, and outgroups, to provide a more detailed phylogenetic hypothesis than previous studies. We analyzed the data using Bayesian methods, increasingly popular in molecular phylogenetics, but hitherto little used in cetacean studies (but see e.g. Yan et al., 2005), in addition to parsimony under various previously proposed weighting schemes.

Given the relatively few characters we certainly acknowledge the limitations of our study, and we did not expect robust clade support, especially for deeper level clades 
that have been consistently contradicted by previous Cytb analyses. However, we set up to test the reliability and sensitivity of our extended Cytb phylogeny based on the recovery of deep level benchmark clades (Euungulata, Perissodactyla, Cetartiodactyla, Cetancodonta, Cetacea, Mysticeti, and Odontoceti). Our study finds: 1) Bayesian phylogenetic methods outperformed parsimony under various models; 2) increased taxon sampling, in particular outgroup sampling (Table 2) increased congruence with other datasets, e.g. for the first time some of our analyses support Odontoceti monophyly based on Cytb data alone.

We find that as long as outgroup taxon sampling was extensive, Bayesian analyses of Cytb recovered all the a priori identified benchmark clades. When only a few outgroups were chosen, however, the Bayesian analysis negated Odontoceti monophyly (Table 2), as have many previous parsimony analyses of mitochondrial DNA. Furthermore, in almost every detailed comparison possible our results mirror the findings O’Leary et al. (2004), the most 'character-complete' (but including relatively few cetacean taxa) analysis to date (37,000 characters from morphology, SINE, and 51 gene fragments). This result gives credibility to our findings, including previously untested lower level clades.

The low support for Odontoceti is unsurprising given previous analysis of Cytb, and the finding of Arnason et al. (2004) that explosive radiation took place early in the evolutionary history of whales, with little time to accumulate synapomorphies for major lineages such as Odontoceti. The parsimony analyses likewise recover the benchmark 
clades Perissodactyla, Cetancodonta, Cetacea, and Mysticeti, but support for Cetartiodactyla and Odontoceti was highly sensitive to a priori character weighting schemes. Using the Arnason and Gullberg (1994) codon weighting scheme (4:17:1), a relatively strong support is found for Odontoceti monophyly. This is an interesting example of how dense taxon sampling can impact the phylogenetic signal. Arnason and Gullberg, (1994) used this weighting scheme in an Cytb analysis of 14 cetacean species and one outgroup (cow) suggested the placement of Mysticeti within Odontoceti. Because Bayesian analyses allows for an objective way of weighting characters (Felsenstein, 1981) and because it recovers all the benchmark clades supported by other independent data (e.g., Messenger and McGuire, 1998; Nikaido et al., 2001; Arnason et al., 2000, 2004; O'Leary et al., 2004) we favor the Bayesian hypothesis. As for other clades, most of the analyses showed remarkable congruence with previous phylogenies based on nuDNA, morphology, and mtDNA data (e.g., Rosel et al., 1995; Messenger and McGuire, 1998; LeDuc et al., 1999; Waddel et al., 2000; Cassens et al., 2001; Hamilton et al., 2001; Rychel et al., 2004; Sasaki et al., 2005). Below we briefly review the implications of our results to lower level cetacean phylogenetics and classification.

\section{Monophyly and placement of Mysticeti (baleen whales)}

The monophyly of baleen whales is virtually uncontroversial (see e.g. Sasaki et al., 2005). However, their placement has been debated. Based on mitochondrial data Milinkovitch et al. $(1993,1995,1994,1996)$ suggested that baleen whales were sister to 
sperm whales (Physeteroidea), Verma et al. (2004) placed them sister to Platanistidae, while Arnason and Gullberg, (1994) based on Cytb placed baleen whales sister to dolphins (however, with very few taxa presented). These hypotheses have remained contradicted by both morphological and nuclear data, which agree on the sister relationship of monophyletic Odontoceti and Mysticeti. Our phylogenetic results agree with morphological and nuclear DNA data (e.g. O'Leary et al., 2004), echoing a new mitogenomic study by Arnason et al. (2004).

Within Mysticeti, we found support for the monophyly of Balaenidae, and the placement of Neobalaenidae sister to (extended) Balaenopteridae. However, Eschrichtius robustus consistently nested within Balaenopteridae, rendering the latter paraphyletic as found by Rychel et al. (2004), O'Leary et al. (2004), and Sasaki et al. (2005).

\section{Monophyly of Odontoceti (toothed whales)}

Odontoceti is one of our benchmark clades, and was supported by the Bayesian analysis and one of the parsimony analyses. The recovery of this clade shows that with sufficient taxa mitochondrial phylogenies can be reliable. Within Odontoceti the superfamilies Delphinoidea, Physeteroidea, and Inoidea were monophyletic, and also all family level taxa (Fig. 1). 


\section{Delphinoids}

All analyses agree on the monophyly of Delphinoidea and monophyly of each of the delphinoid families Monodontidae, Phocoenidae, and Delphinidae, and all subfamilies within Delphinidae. Our results strongly support the relationship Delphinidae (Monodonotidae + Phocoenidae). Waddel et al. (2000) found the same relationships with nuclear genes and Nishida et al. (2003) with SRY (sex determining region of the $\mathrm{Y}$ chromosome) gene. Our findings contradict the division of Phocoenidae into two subfamilies Phocoeninae and Phocoenoidinae. The porpoises Australophoceona dioptrica and Phoecoenoides dalli, rather nested within Phocoeninae, and Neophocaena phocaenoides is basal to all the porpoises. As Rosel et al. (1995) suggested, Australophocoena should be returned to Phocoena where it was originally placed by Lahille (1912), and Phocoenoies dalli classification needs further analysis.

LeDuc et al. (1999) and LeDuc (2002) proposed a new classification for Delphinidae based on Cytb data. Unsurprisingly, our results largely agree. Stenella and Lagenorhynchus are paraphyletic and both need revision. Grampus griseus nested within the subfamily Globicephalinae in all our analyses (see also Kasuya, 1973), rather than within Delphininae as previously suggested (Mead, 1975; Barnes et al., 1985; De Muizon, 1988; Perrin, 1989). Orcininae (Orcinus orca + Orcaella brevirostris), separate from Globicephalinae is supported. Sousa chinensis groups within the subfamily Delphininae and not with Stenoninae. Furthermore, our results show a monophyletic Lissodelphininae including Cephalorhynchus spp., Lissodelphis spp., and 
Lagenorhynchus australis, L. cruciger, L. obliquidens and L. obscurus. As suggested by previous studies Lagenorhynchus is not monophyletic. Our results support LeDuc et al. (1999) in transferring Lagenorhynchus acutus to Leucopleurus, but its phylogenetic position also requires the creation of a new subfamily, likely also including L. albirostris. LeDuc et al. (1999) and LeDuc (2002) suggested returning these four species to the genus Sagmatias (type species L. cruciger), however, in our analyses $L$. cruciger and $L$. australis are nested within Cephalorhynchus. Thus it may be simplest to transfer the $L$. cruciger and $L$. australis to Cephalorhynchus, while retaining $L$. obliquidens and $L$. obscurus in Lagenorynchus. Interestingly, the placement of L. australis within Cephalorhynchus is supported by acoustic data. Uniquely among dolphins, L. australis, and the four Cephalorhynchus species do not whistle (Schevill and Watkins, 1971). There is not published data on the acoustic behavior of L. cruciger.

River Dolphins

Our results agree with most molecular and recent morphological studies that river dolphins are polyphyletic, and do not offer unambiguous support for the infraorder Delphinida (containing Delphinoidea, Lipotidae, Iniidae, Pontoporidae, Platanistidae). As suggested by most studies Platanistidae does not group with other river dolphins, but is here the most basal family of Delphinida (e.g., Messenger and McGuire, 1998; Cassen et al., 2000; Hamilton et al., 2001; Yan et al., 2005). Note that a recent study based on nuDNA and Cytb placed Platanistidae sister to Mysticeti, although with little support 
(Verma et al., 2004). Platanista is the only surviving genus of the superfamily

Platanistoidea which contains the extinct marine families Prosqualodontidae,

Dalpiazinidae, Waipatiidae, Squalondontidae, Squalodelphinidae, in addition to

Platanistidae (deMuizon, 2002). Although, paleontologists agree that Platanista is a close relative of the family Squalodelphinidae (Heyning, 2002) new paleontological data points to Lipotes vexillifer and Inia geoffrensis as its closest relatives (Geisler and Sanders, 2003).

Geisler and Sanders, (2003) suggest a single ecological shift to riverine habitats in the 'river dolphins', instead of two as argued by other authors (e.g., Cassens et al., 2000; Nikaido et al., 2001; Hamilton et al., 2001). Our results indicate two to three shifts in the 'river dolphins'. An unambiguous one in Platanista, and either one in Inia and another in Lipotes, or a single origin in the node leading to Inoidea plus Lipotoidea with a reversal in Pontoporia. In addition, populations of Sotalia fluviatilis, Orcaella brevirostris (LeDuc, 1999), and the porpoise Neophocaena phocaenoides, independently shifted to a riverine habitat.

Previously the three 'river dolphin' genera were placed in a single family Iniidae (Heyning, 1989) or two families Pontoporidae (Pontoporia and Lipotes) and Innidae (Inia) (Fordyce et al., 1994). Our phylogenetic results agree with the classification of the three genera into three families as suggested by Fordyce and deMuizon (2001) with the following relationship ((Pontoporidae + Iniidae $)+$ Lipotidae $))$. This arrangement is supported by both morphology and molecular data (e.g., Messenger and McGuire, 1998; 
Yang and Zhou 1999; Cassen et al., 2000; Hamilton et al., 2001; Nikaido et al., 2001; Yan et al., 2005). Furthermore, the relationship of Inia subspecies is unsurprisingly identical to that found by Hamilton et al., (2001) (I. g. humboldtiana + I. g. geoffrensis) + I. g. boliviensis)).

\section{Beaked and sperm whales}

Our results support the superfamily Physeteroidea which includes the families Kogiidae and Physeteridae, whereas ziphiids interrelationships were largely unresolved. The molecular work of Dalebout et al. (2004) calls for a revision of Ziphiidae and Mead (2002) proposed the subfamilies Ziphininae (Berardius spp., Tasmacetus shepherdi, and Ziphius cavirostris) and Hyperoodontinae (Mesoplodon spp. and Hyperoodon spp). Our analyses all indicate Tasmacetus shepherdi sister to all other ziphiids. To date, most cetacean phylogenies have not aimed at solving ziphiid species relationships, and thus their relationships are largely unknown. Since low level taxonomic relationships were fairly well supported in other groups of toothed whale, Cytb seems promising in providing future insights in the evolutionary relationships of ziphiids. Physeteroids and ziphiids are the most basal toothed whales. Both groups show a clear reduction in dentition, in physeteroids teeth are only present in the lower jaw, and in most ziphiid species, teeth are reduced or absent in both jaws, with the exception of males that have two prominent teeth in the lower jaw (Mead 2002). 
It is interesting to notice that $T$. sheperdi is basal in our ziphiid phylogeny and it is the only beaked whale with full dentition in both jaws. Although this particular relationship is weakly supported, it hints that the loss of teeth may be convergent in Physeteroidea, and within Ziphiidae. 


\section{CONCLUDING REMARKS}

Substitution saturation imposes limitation on Cytb (and other mitochondrial data) for deeper level phylogenetics, and may lead to misleading results (Irwin et al. 1991; Springer et al. 2001). Furthermore, many studies have shown that single gene analyses rarely agree with global optima (e.g., O’Leary et al., 2004). However, our results show that by densely sampling taxa, especially outgroup taxa, and using appropriate methods of analysis with realistic models of evolution, this problem may be reduced, and in this particular example, mostly overcome. Low-level phylogenies are essential for classification and as a tool for comparative evolutionary (and ecological) studies. In this context 'single gene' phylogenies may be of great value (as long as they are 'reality checked') as relatively many species can be included, offering more detailed phylogenies than currently possible with phylogenies based on multiple genes and morphology. Ultimately, of course, a major goal of phylogenetics is a phylogeny of life (i.e. many taxa), based on multiple lines of evidence (many characters of many types). However, when phylogenies based on relatively few characters can be judged reliable based on external evidence (taxonomic congruence with other phylogenies using many characters, but few taxa), they seem like very promising and useful 'first guess' hypotheses. The evolution of sexual dimorphism, echolocation, social behavior, and whistles and other communicative signals, and major ecological shifts (e.g. transition to fresh water) are among the numerous interesting questions in cetacean biology that this phylogeny can help answer. 


\section{ACKNOWLEDGEMENTS}

We thank Douglas Wartzok, Timothy Collins, Agnar Ingólfsson, Jim McGuire, and an anonymous reviewer for helpful comments on a version of the manuscript. Wayne P. Maddison helped with data analyses and provided computational support. Support for this study came to Laura May-Collado from Tinker Research Opportunities Award, American Natural History Museum (Lener-Gray Award), Animal Behavior Society (Cetacean Behavior and Conservation Award), STRI, and Project Aware and a Killam Postdoctoral Fellowship to Ingi Agnarsson. 


\section{LIST OF REFERENCES}

Arnason, U., Gretarsdottir, S., Widegren, B., 1992. Mysticete (baleen whale) relationships based upon the sequence of the common cetacean DNA satellite. Mol. Biol. Evol. 9, 1018-1028.

Arnason, U., Gullberg, A., Widegren, B., 1993. Cetacean mitochondrial DNA control region: sequences of all extant baleen whales and two sperm whale species. Mol. Biol. Evol. 10, 960-970.

Arnason, U., Gullberg, A., 1993. Comparison between the complete mtDNA sequences of the blue and the fin whale, two species that canhybridize in nature. J. Mol. Evol. 37, $312-322$.

Arnason, U., Gullberg, A., 1994. Relationship of baleen whales established by cytochrome b gene comparison. Nature 367, 726- 728.

Arnason, U., Gullberg, A., 1996. Cytochrome b nucleotide sequences and the identification of five primary lineages of extant cetaceans. Mol. Biol. Evol. 13, 407- 417.

Arnason, U., Gullberg, A., Gretarsdottir, S., Ursing, B., Janke, A., 2000. The complete mitochondrial genome of the sperm whale and the establishment of a new molecular reference for estimating eutherian divergence dates. J. Mol. Evol. 50, 569- 578.

Arnason, U., Gullberg, A., Janke, A., 2004. Mitogenomic analyses provide new insights into cetacean origin and evolution. Gene 333, 27-34.

Adachi, J., Hasegawa, M., 1995. Phylogeny of whales: dependence of the inference on species sampling. Mol. Biol. Evol. 12, 177-179.

Boisserie J. R., Lihoreau, F, Brunet, M., 2005. The position of Hippopotamidae within Cetartiodactyla. Proc. Natl. Acad. Sci. 102, 1537-1531.

Barnes, L.G., Domning, D.P., Ray, C.E., 1985. Status of studies on fossil marine mammals. Mar. Mamm. Sci. 1, 15-53.

Cassens, I., Vicario, S., Waddell, V.G., Balchowsky, H., Van Belle, D., Ding, W., Fan, C., Lal Mohan, R.S., Simões-Lopes, P.C., Bastida, R., Meyer, A., Stanhope, M.J., Milinkovitch, M.C., 2000. Independent adaptation to riverine habitats allowed survival of ancient cetacean lineages. Proc. Natl. Acad. Sci. 97, 11343-11347.

Cerchio, S., Tucker, P., 1998. Influence of aligment on the mtDNA phylogeny of 
Cetacea: questionable support for a Mysticeti/Physeteroidea clade. Syst. Biol. 47, 336344.

Cozzuol, M.A., 1985. The Odontoceti of the Mesopotamiense of he Parana River ravines. Invest. Cetacea 7, 39-54.

Dalebout, M.L., Baker, C.S., Mead, J.G., Cockcroft, V.G., Yamada, T.K., 2004. A comprehensive and validated molecular taxonomy of beaked whales, Family Ziphiidae. J. Hered. 95, 159-173.

deMuizon, C., 1988. Les relations phylogogenetiques des Delphinida (Cetacea, Mammalia). Ann. Paleontol. 74, 159-227.

deMuizon, C., 2002. River dolphins, Evolutionary History. Encyclopedia of Marine Mammals. Perrin, W.F., Würsig, B., Thewissen, J.G.M. (Eds). Academic Press, pp. 1043-1049.

Douzery, E., 1993. Evolutionary relationships among Cetacea based on the sequences of the mitochondrial 12S rRNA gene: possible paraphyly of toothed-whales (odontocetes) and long separate evolution of sperm whales (Physeteridae). C. R. Acad. Sci. Paris, Life Sciences, Evolution. 316, 1515-1518.

Felsenstein, J., 1981. Evolutionary trees from DNA-sequences - a maximum-likelihood approach. J. Mol. Evol. 17, 368-376.

Felseinstein, J., 1985. Confidence limits on phylogenies with a molecular clock. Syst. Zool. 34, 152-161.

Flower, W.F., 1867. Description of the skeleton of Inia geoffrensis. Trans. Zool. Soc. London 6, 87-116.

Fordyce, R.E., Barnes, L.G., Miyazaki, N., 1994. General aspects of the evolutionary history of whales and dolphins. Island Arct. 3, 373-391.

Fordyce, R.E., deMuizon, C., 2001. Evolutionary history of Cetaceans: A review. In Secondary adaptation to life in the water. deBuffrenil, V., Mazin, J.M. (Eds). Pfeil Verlag, Munich, pp. 163-233.

Gatesy, J., 1997. More DNA support for a Cetacea/Hippopotamidae clade: the bloodclotting protein gene $\sqrt{ }$-fibrinogen. Mol. Biol. Evol. 14, 537-543. 
Gatesy, J., Hayashi, C., Cronin, M.A., Arctander, P., 1996. Evidence from milk casein genes that cetaceans are close relatives of hippopotamid artiodacyls. Mol. Biol. Evol. 13, 954-963.

Gatesy, J., Milinkovitch, M., Waddell, V., Stanhope, M., 1999. Stability of cladistic relationships between Cetacea and higher-level Artiodactyl taxa. Syst. Biol. 48, 6-20.

Geisler, J.H., Sanders, A.E., 2003. Morphological evidence for the phylogeny of Cetacea. J. Mammal. Evol. 10, 23-129

Gingerich, P.D., Smith, B.H., Simons, E.L., 1990. Hind limbs of Eocene Basilosaurus: evidence of feet in whales. Science 249, 154-157.

Goloboff, P., 1993. NONA Version 1.9. Computer program and documentation http://www.cladistics.com.

Graur, D., Higgins, D.G., 1994. Molecular evidence for the inclusion of Cetaceans within the Order Artiodactyla. Mol. Biol. Evol. 11, 357-364.

Graybeal, A., 1998. Is it better to add taxa or characters to a difficult phylogenetic problem. Syst. Biol. 47, 9-17.

Hamilton, H., Caballero, S., Collins, A.G., Brownell, R.L., 2001. Evolution or river dolphins. Proc. R. Soc. Lond. B. 268, 549-558.

Hasegawa, M., Adachi, J., 1996. Phylogenetic position of Cetaceans relative to Artiodactyls: reanalysis of mitochondrial and nuclear sequences. Mol. Biol. Evol. 13, 710-717.

Huelsenbeck, J.P., Ronchist, F., 2001. MRBAYES: Bayesian inference of phylogenetic trees. Bioinformatics. 17, 754-755.

Hillis, D.M., 1996 Inferring complex phylogenies. Nature 383, 130-131.

Hillis, D.M., 1998. Taxonomic sampling, phylogenetic accuracy, and investigator bias. Syst. Biol. 47, 3-8.

Hillis, D.M., Pollock, D.D., McGuire, J.A., Zwick, D.J., 2003. Is Sparse Taxon Sampling a Problem for Phylogenetic Inference? Syst. Biol. 52, 124-126.

Heyning, J., 2002. River dolphins, relationships. Encyclopedia of Marine Mammals. 
Perrin, W.F., Würsig, B., Thewissen, J.G.M. (Eds). Academic Press, pp. 1050-1051. Irwin, D.M., Arnason, U., 1994. Cytochrome b gene of marine mammals: phylogeny and evolution. J. Mamm. Evol. 2, 37-55.

Irwin, D.M., Kocher, T.D., Wilson, A.C., 1991. Evolution of the Cytochrome b of Mammals. J. Mol. Evol. 32, 128-144.

Kasuya, T., 1973. Systematic consideration of recent toothed whales based on the morphology of tymapano-periotic bone. Sci. Rep. Whales Res. Inst. 25, 1-103.

LeDuc, R.G., Perrin, W.F., Dizon, A.E.,1999. Phylogenetic relationships among the delphinid cetaceans based on full cytochrome b sequences. Mar. Mamm. Sci. 15, 619648.

LeDuc, R., 2002. Delphinids overview. Encyclopedia of Marine Mammals. Perrin, W.F., Würsig, B., Thewissen, J.G.M. (Eds). Academic Press, pp. 310-314.

28

Luckett, W.P., Hong, N., 1998. Phylogenetic relationships between the orders Artiodactyla and Cetacea: a combined assessment of morphological and molecular evidence. J. Mammal. Evol. 5, 127-182.

Lum, J. K., Nikaido, M., Shimamura, M., Shimodaira, H., Shedlock, A.M., Okada, N., Hasegawa, M., 2000. Consistency of SINE insertion topology and flanking sequence tree: quantifying relationships among Cetartiodactyls. Mol. Biol. Evol. 17, 1417-1424.

Maddison, W.P., Maddison, D.R. 2003. MacClade: Analysis of Phylogeny and Character Evolution. Version 4.07. Sunderland, Massachusetts: Sinauer Associates.

Matthee, C.A., Burzlaff, J.D., Taylor, J.F., Davis, S.K., 2001. Mining the Mammalian Genome for Artiodactyl Systematics. Syst. Biol. 50, 367-390.

Mead, J.G., 1975. Anatomy of the external nasal passages and facial complex in the Delphinidae (Mammalia:Cetacea). Smithson. Contr. Zool. 207, 1-72.

Mead, J. G., 2002. Beaked whales overview. Encyclopedia of Marine Mammals. Perrin, W.F., Würsig, B., Thewissen, J.G.M. (Eds). Academic Press, pp. 81-84.

Messenger, S.L., McGuire, J.A., 1998. Morphology, Molecules, and the Phylogenetics of Cetaceans. Syst. Biol. 47, 90-124. 
Milinkovitch, M.C., 1995. Molecular phylogeny of cetaceans prompts revision of morphological transformations. Trends Ecol. Evol. 10, 328-334.

Milinkovitch, M.C., 1997. The phylogeny of whales: a molecular approach. In Molecular genetics of marine mammals. Dizon, A.E., Chivers, S.J., Perrin, W.F. (Eds). The Society for Marine Mammalogy. Special Publication 3, 317-338

Milinkovitch, M.C, Orti, G., Meyer, A., 1993. Revised phylogeny of whales suggested by mitochondrial ribosomal DNA sequences. Nature 361, 346-348.

Milinkovitch, M.C., Lyons-Weiler, J., 1997. Finding Optimal Ingroup Topologies and Convexities When the Choice of Outgroups Is Not Obvious. Mol. Phylogenet. Evol. 9, 348-357.

Milinkovitch, M.C, Meyer, A., Powell, J.R., 1994. Phylogeny of all major groups of cetaceans based on DNA sequences from three mitochondrial genes. Mol. Biol. Evol. 11, 939-948.

Milinkovitch, M.C, Orti, G., Meyer, A., 1995. Novel phylogeny of whales revisited but not revised. Mol. Biol. Evol. 12, 518-520.

Milinkovitch, M.C., LeDuc, R.G., Adachi, J., Farnir, F., Georges, M., Hasegawa, M., 1996. Effects of character weighting and species sampling on phylogeny reconstructuion: a case study based on DNA sequence data in Cetaceans. Genetics 144, 1817-1833.

Miller, J.A., G. Hormiga., 2004. Clade stability and the addition of data: A case study from erigonine spiders (Araneae: Linyphiidae, Erigoninae). Cladistics, 20, 385-442.

Montgelard, C., Catzeflis, F.M., Douzery, E., 1997. Phylogenetic relationships of Artiodactyls and Cetaceans as deduced from the comparison of cytochrome $b$ and $12 \mathrm{~S}$ rRNA mitochondrial sequences. Mol. Biol. Evol. 14, 550-559.

Murphy, W.J., Elzirik, E., Johnson, W.E., Zhang, Y.P., Ryder, O.A., O’Brien, S.J., 2001. Molecular phylogenetics and the origins of placental mammals. Nature 409, 614-618.

Nikaido, M., Matsuno, F., Hamilton, H., Brownell, R.L., Cao, Y., Ding, W., Zuoyan, Z., Shedlock, A.M., Fordyce, R.E., Hasegawa, M., Okada, N., 2001. Retroposon analysis of major cetacean lineages: The monophyly of toothed whales and the paraphyly of river dolphins. Proc. Natl. Acad. Sci. 98, 7384-7389.

Nikaido, M., Rooney, A.P., Okada, N., 1999. Phylogenetic relationships among cetartiodactyls based on insertions of short and long interspaced elements: 
Hippopotamuses are the closest extant relative of whales. Proc. Natl. Acad. Sci. 96, 10261-10266.

Nishida, S., Pastene, L.A., Goto, M., Koike, H., 2003. SRY gene structure and phylogeny in the cetacean species. Mammal Study 28, 57-66.

Nixon, K.C., 2002. WinClada, ver. 1.00.08. K.C. Nixon, Ithaca, NY. Available: http://www.cladistics.com.

O’Leary, M.A, Geisler, J.H., 1999. The position of Cetacea within Mammalia: phylogenetic analysis of morphological data from extinct and extant taxa. Syst. Biol. 48, 455-490.

O’Leary, M.A., M., Allard, M.J., Novacek, M.J., Meng, J., Gatesy, J., 2004. Building the mammalian sector of the tree of life: combining different data and a discussion of divergence times of placental mammals. In Assembling the Tree of Life. Chapter 28. Cracraft, J., Donoghue, M.J. (eds), Oxford University Press, pp. 490-516.

Perrin, W.F., 1989. Dolphins, porpoises, and whales. An action plan for the conservation of biological diversity: 1989-1992. Second edition. IUCN, Gland, Switzerland. 27 Pp.

Pichler, F.B., Robineau, D., Goodall, R.N.P., Meyer, M.A., Olivarria, C., 2001. Origin and radiation of southern hemisphere coastal dolphins (genus Cephalorhynchus). Mol. Ecol. 10, 2215-2223.

Pollock, D.D., Zwichkl, D.J., McGuire, J.A., Hillis, D.M., 2002. Increased taxon sampling is advantageous for phylogenetic inference. Syst. Biol. 51, 664-671.

Posada, D., Crandall, K.A., 1998. Modeltest: testing the model of DNA substitution. Bioinformatics 14, 817-818.

Posada, D., Crandall, K.A., 2001. Selecting the Best-Fit Model of Nucleotide Substitution. Syst. Biol. 50, 580 - 601

Posada, D., Buckley, T.R., 2004. Model selection and model averaging in phylogenetics: advantages of akaike information criterion and bayesian approaches over likelihood ratio tests. Syst Biol . 53, 793-808

Reyes, A., Giss, C., Catzeflis, F., Nevo, E., Pesole, G., Saccone, C., 2004. Congruent mammalian trees from mitochondrial and nuclear genes using Bayesian methods. Mol. Biol. Evol. 21, 397-403. 
Rodríguez, F., Oliver, J.F., MarÍn, A., Medina, J.R., 1990. The general stochastic model of nucleotide substitution. J. Theor. Biol. 142, 485-501.

Rosel, P.F., Haygood, M.G., Perrin, W.F., 1995. Phylogenetic relationships among the true porpoises (Cetacea:Phocoenidae). Mol. Phylogenet. Evol. 4, 463-474.

Rosenberg, M.S., Kumar., S., 2001. Incomplete taxon sampling is not a problem for phylogenetic inference. Proc. Natl. Acad. Sci. 98, 10751-10756.

Rosenberg, M.S., Kumar, S., 2003. Taxon sampling, bioinformatics, and phylogenomics. Syst. Biol. 52, 119-124.

Rokas, A., Caroll, S.B., 2005. More Genes or more taxa? The relative contribution of gene number and taxon number to phylogenetic accuracy. Mol. Biol. Evol. Advanced access online.

Rychel, A.L., Reeder, T.W., Berta, A., 2004. Phylogeny of mysticete whales based on mitochondrial and nuclear data. Mol. Phylogenet. Evol. 32, 893-901.

Sasaki, T., Nikaido, M., Hamilton, H., Goto, M., Kato, H., Kanda, N., Pastene, L.A., Cao, Y., Fordyce, E., Hasegawa, M., Okada, N., 2005. Mitochondrial Phylogenetics and Evolution of Mysticete Whales. Sys. Biol. 54, 77 - 90

Schevill, W.E., Watkins, W.A., 1971. Pulsed sounds of the porpoise Lagenorhynchus australis. Breviora 366, 1-10.

Shimura, A.M., Yasue, H., Ohshima, K., Abe, H., Kato, H., Kishiro, T., Goto, M., Munechika, I., Okada, N., 1997. Molecular evidence from retroposons that whales form a clade within even-toed ungulates. Nature 388, 666-670.

Shimamura, M., Hideaki, A., Nikaido, M., Ohshima, K., Okada, N., 1999. Mol. Biol. Evol. 16, 1046-1060.

Simpson, G.G., 1945. The principles of classification and a classification of mammals. Bull. Am. Mus. Nat. Hist. 85, 1-350.

Slijper, E., 1936. Die Cetacea vergleichend-anatomish und systematisch. Capita Zool. 7, $1-590$.

Springer, M.S., DeBry, R.W., Douady, C., Amrine, H.M., Madsen, O., de Jong, W.W., Stanhope, M.J., 2001. Mitochondrial versus nuclear gene sequences in deep-level mammalian phylogeny reconstruction. Mol. Biol. Evol. 18, 132-143. 
Thewissen, J.G.M., Madar, S.I., 1999. Ankle morphology of the earliest Cetaceans and its implications for phylogenetic relationships among ungulates. Syst. Biol. 48, 21-30.

Thewissen, J.G.M., Williams, E.M., Roe, L.J., and Hussain, S.T., 2001. Skeletons of terrestrial cetaceans and the relationship of whales to artiodactyls. Nature 413, 277-281.

Verma, S.N., Sinha, R.K., Singh, L., 2004. Phylogetic position of Platanista gangetica: insights from the mitochondrial cytochrome $b$ and nuclear interphotoreceptor retinoidbinding protein gene sequences. Mol. Phylogenet. Evol. 33, 280-288.

Waddell, V.G., Milinkovitch, M.C., Bérubé, M., Stanhope, M.J., 2000. Molecular phylogenetic examination of the Delphinoidea trichotomy: congruent evidence from three nuclear loci indicates that porpoises (Phocoenidae) share a more recent common ancestry with white whales (Monodontidae) than they do with true dolphins (Delphinidae). Mol. Phylogenet. Evol. 15, 314-318.

Waddell P.J., Kishino H., Ota R., 2001. A phylogenetic foundation for comparative mammalian genomics. Genome Informatics 12, 141-154.

Winge, H., 1921. A review of the interratationships of the Cetacea. Smithsonian Misc. Collect. 72, 1-97.

Yan, J, Zhou, K, Yang, G. 2005. Molecular phylogenetics of 'river dolphins' and the baiji mitochondrial genome. Mol. Phylogenet. Evol. In Press (online).

Yang, Z., 1994. Maximum likelihood phylogenetic estimation from DNA sequences with variable rates over sites: approximate methods. J. Mol. Evol. 39, 306-314.

Yang, G., Zhou, K., 1999. A study on the molecular phylogeny of river dolphins. Acta Theriol. Sinica, 19, 1-9.

Zwickl, D.J., Hillis, D.M., 2002. Increased taxon sampling greatly reduces phylogenetic error. Syst. Biol. 51, 588-598. 
Table 1. Species used in the analyses with respective GenBank accession numbers of cytochrome b sequences.

\begin{tabular}{|c|c|c|c|}
\hline \multicolumn{2}{|c|}{ All species (Bayesian + Parsimony) } & \multirow[t]{2}{*}{ Subsample $^{1}$} & \multirow[t]{2}{*}{ Subsample } \\
\hline Species & Accession \# & & \\
\hline \multicolumn{4}{|l|}{ Carnivora } \\
\hline Canis familiaris & AY729880 & & $\mathrm{X}$ \\
\hline Panthera leo & AF053052 & & $\mathrm{X}$ \\
\hline \multicolumn{4}{|l|}{ EUUNGULATA } \\
\hline \multicolumn{4}{|l|}{ Perissodactyla } \\
\hline $\begin{array}{l}\text { Dicerorhinus } \\
\text { sumatrensis }\end{array}$ & AJ245723 & & $\mathrm{X}$ \\
\hline D. bicornis & X56283 & & $\mathrm{X}$ \\
\hline Equus caballus & AY515162 & & $\mathrm{X}$ \\
\hline Equus grevyi & X56282 & & $\mathrm{X}$ \\
\hline Tapirus indicus & AF145734 & & $\mathrm{X}$ \\
\hline T. terrestris & AF056030 & & $\mathrm{X}$ \\
\hline \multicolumn{4}{|l|}{ Cetartiodactyla } \\
\hline Alces alces & M98484 & & $\mathrm{X}$ \\
\hline Antilocapra americana & AF091629 & $\mathrm{X}$ & $\mathrm{X}$ \\
\hline Antilope cervicapra & AF022058 & & $\mathrm{X}$ \\
\hline Babyrousa babyrussa & Z50106 & & $\mathrm{X}$ \\
\hline Bos Taurus & AB090987 & $\mathrm{X}$ & $\mathrm{X}$ \\
\hline Camelus dromedarius & X56281 & $\mathrm{X}$ & $\mathrm{X}$ \\
\hline Cephalophus zebra & AF153903 & & $\mathrm{X}$ \\
\hline $\begin{array}{l}\text { Giraffa } \\
\text { camelopardalis }\end{array}$ & X56287 & & $\mathrm{X}$ \\
\hline Lama lama & U06429 & & $\mathrm{X}$ \\
\hline Moschus leucogaster & AF026889 & & $\mathrm{X}$ \\
\hline Odocoileus hemionus & X56291 & & $\mathrm{X}$ \\
\hline Oryx gazelle & AF249973 & & $\mathrm{X}$ \\
\hline Ovis aries & AB006800 & & $\mathrm{X}$ \\
\hline Pecari tajacu & X56296 & $\mathrm{X}$ & $\mathrm{X}$ \\
\hline Sus barbatus & Z50107 & & $\mathrm{X}$ \\
\hline \multicolumn{4}{|l|}{ CETANCODONTA } \\
\hline $\begin{array}{l}\text { Hippopotamus } \\
\text { amphibius }\end{array}$ & Y08813 & & \\
\hline \multicolumn{4}{|l|}{ Cetacea } \\
\hline \multicolumn{4}{|l|}{ Mysticeti } \\
\hline Balaena glacialis & $\mathrm{X} 75587$ & & \\
\hline Balaena mysticetus & U13125 & $\mathrm{X}$ & $\mathrm{X}$ \\
\hline $\begin{array}{l}\text { Balaenoptera } \\
\text { bonaerensis }\end{array}$ & $\mathrm{X} 75581$ & $\mathrm{X}$ & \\
\hline
\end{tabular}




\begin{tabular}{|c|c|c|c|}
\hline B. borealis & $\mathrm{X} 75582$ & & \\
\hline B. edeni & $\mathrm{X} 75583$ & & \\
\hline Capera marginata & $\mathrm{X} 75586$ & & \\
\hline Eschrichtius robustus & $\mathrm{X} 75585$ & $\mathrm{X}$ & $\mathrm{X}$ \\
\hline $\begin{array}{l}\text { Megaptera } \\
\text { novaeangliae }\end{array}$ & X75584 & $\mathrm{X}$ & $\mathrm{X}$ \\
\hline \multicolumn{4}{|l|}{ Odontoceti } \\
\hline \multicolumn{4}{|l|}{ Physeteroidea } \\
\hline \multicolumn{4}{|l|}{ Physeteridae } \\
\hline $\begin{array}{l}\text { Physeter } \\
\text { macrocephalus } \\
\text { (catodon) }\end{array}$ & X75589 & $\mathrm{X}$ & $\mathrm{X}$ \\
\hline \multicolumn{4}{|l|}{ Kogidae } \\
\hline Kogia breviceps & U72040 & $\mathrm{X}$ & $\mathrm{X}$ \\
\hline Kogia simus & AF304072-U13135 & $\mathrm{X}$ & $\mathrm{X}$ \\
\hline \multicolumn{4}{|l|}{ Ziphoidea } \\
\hline \multicolumn{4}{|l|}{ Ziphiidae } \\
\hline Berardius bairdii & $\mathrm{X} 92541$ & & \\
\hline Hyperoodon planifrons & AY579560 & & \\
\hline H. ampullatus & AY579558 & & \\
\hline Indopacetus pacificus & AY162441 & & \\
\hline $\begin{array}{l}\text { Mesoplodon } \\
\text { densirostris }\end{array}$ & X92536 & $\mathrm{X}$ & \\
\hline M. bidens & X92538 & $\mathrm{X}$ & \\
\hline Tasmacetus shepherdi & AF334484 & & \\
\hline Ziphius cavirostris & AF304075-U13146 & $\mathrm{X}$ & $\mathrm{X}$ \\
\hline \multicolumn{4}{|l|}{ Platanistoidea } \\
\hline \multicolumn{4}{|l|}{ Platanistidae } \\
\hline Platanista gangetica & AF304070 & & \\
\hline Platanista minor & X92543 & & \\
\hline \multicolumn{4}{|l|}{ Inoidea } \\
\hline \multicolumn{4}{|l|}{ Pontoporidae } \\
\hline Pontoporia blainvelli & AF334488 & & \\
\hline Iniidae & AF334487 & & \\
\hline $\begin{array}{l}\text { Inia geoffrensis } \\
\text { boliviensis }\end{array}$ & AF334485 & $\mathrm{X}$ & $\mathrm{X}$ \\
\hline $\begin{array}{l}\text { Inia geoffrensis } \\
\text { geoffrensis }\end{array}$ & AF521110 & & \\
\hline \multicolumn{4}{|l|}{$\begin{array}{l}\text { Inia geoffrensis } \\
\text { humboldtiana }\end{array}$} \\
\hline Lipotoidea & & & \\
\hline
\end{tabular}




\begin{tabular}{|c|c|c|c|}
\hline Lipotidae & & & \\
\hline Lipotes vexillifer & AF304071 & & \\
\hline Delphinoidea & & & \\
\hline Monodontidae & & & \\
\hline Delphinapterus leucas & U72037 & $\mathrm{X}$ & $\mathrm{X}$ \\
\hline Monodon monocerus & U72038 & & \\
\hline Phocoenidae & & & \\
\hline $\begin{array}{l}\text { Australophocoena } \\
\text { dioptrica }\end{array}$ & U09681 & & \\
\hline $\begin{array}{l}\text { Neophocaena } \\
\text { phocaenoides }\end{array}$ & AF334489 & & \\
\hline Phocoena phocoena & U72039 & $\mathrm{X}$ & $\mathrm{X}$ \\
\hline P. sinus & AF084051 & & \\
\hline P. spinipinnis & U09676 & $\mathrm{X}$ & $\mathrm{X}$ \\
\hline Phocoenoides dalli & U09679 & & \\
\hline Delphinidae & & & \\
\hline $\begin{array}{l}\text { Cephalorhynchus } \\
\text { commersonii }\end{array}$ & AF084073 & & \\
\hline C. eutropia & AF084072 & $\mathrm{X}$ & $\mathrm{X}$ \\
\hline C. hectori & AF084071 & & \\
\hline C. heavisidii & AF084070 & & \\
\hline Delphinus delphis & AF084085 & $\mathrm{X}$ & $\mathrm{X}$ \\
\hline D. capensis & AF084087 & & \\
\hline D. tropicalis & AF084088 & & \\
\hline Feresa attenuata & AF084052 & & \\
\hline $\begin{array}{l}\text { Globicephala } \\
\text { macrorhynchus }\end{array}$ & AF084055 & & \\
\hline G. melas & AF084056 & $\mathrm{X}$ & $\mathrm{X}$ \\
\hline Grampus griseus & AF084059 & & \\
\hline $\begin{array}{l}\text { Lagenorhynchus } \\
\text { acutus }\end{array}$ & AF084075 & & \\
\hline L. australis & AF084069 & & \\
\hline L. cruciger & AF084068 & & \\
\hline L. obliquidens & AF084067 & $\mathrm{X}$ & \\
\hline L. obscurus & AY257161 & $\mathrm{X}$ & $\mathrm{X}$ \\
\hline Lagenodelphis hosei & AF084099 & & \\
\hline Lissodelphis borealis & AF084064 & & \\
\hline L. peronii & AF084065 & $\mathrm{X}$ & $\mathrm{X}$ \\
\hline Orcinus orca & AF084061 & & \\
\hline Orcaella brevirostris & AF084063 & & \\
\hline Pseudorca crassidens & AF084057 & & \\
\hline Stenella attenuata & AF084096 & & \\
\hline S. clymene & AF084083 & & \\
\hline S. coeruleoalba & AF084082 & & \\
\hline S. frontalis & AF084090 & & \\
\hline
\end{tabular}




\begin{tabular}{|l|l|l|l|}
\hline S. longirostris & AF084103 & & \\
\hline Sousa chinensis & AF084080 & & \\
\hline Sotalia fluviatilis & AF304067 & & \\
\hline Steno bredanensis & AF084077 & & \\
\hline Tursiops truncatus & AF084095 & X & X \\
\hline
\end{tabular}

Note: In subsample 1 species were included to mirror taxon selection in Messenger and McGuire (1998) molecular analyses except for the outgroup species Tragulus napu.

Furthermore, the following cetacean species were replaced by close relatives: Mesoplodon europaeus and M. peruvianus by M. bidens and M. densirostris, Lagenorhynchus albirostris by L. obliquidens, and Balaenoptera physalus by $B$.

bonaerensis. In subsample 2 the same species were included as in subsample 1 plus all outgroups from this study. 
Table 2. Posterior probabilities and bootstrap values for all benchmark clades

\begin{tabular}{|c|c|c|c|c|c|c|c|}
\hline \multirow[b]{2}{*}{ Model } & \multicolumn{7}{|c|}{ Benchmark clades } \\
\hline & Euungulata & Perissodactyla & Cetartiodactyla & Cecantodonta & Cetacea & Mysticeti & Odontoceti \\
\hline $\begin{array}{l}\text { Bayesian } \\
\text { GTR+ }+\Gamma+I\end{array}$ & 100 & 100 & 100 & 79 & 100 & 100 & 67 \\
\hline $\begin{array}{l}\text { Subsample } \\
\text { Bayesian }\end{array}$ & $\mathrm{n} / \mathrm{a}$ & $\mathrm{n} / \mathrm{a}$ & $\mathrm{n} / \mathrm{a}$ & $\mathrm{n} / \mathrm{a}$ & 100 & 98 & $\mathrm{NO}$ \\
\hline $\begin{array}{l}\text { Subsample } \\
\text { w/outgr. }\end{array}$ & 100 & 100 & 100 & 71 & 100 & 99 & 78 \\
\hline $\begin{array}{l}\text { Equally } \\
\text { Weigthed } \\
\text { MP }\end{array}$ & 100 & 100 & $\mathrm{NO}$ & $<50$ & 100 & 100 & NO \\
\hline MP (3:1) & 100 & 100 & $\mathrm{NO}$ & 100 & 100 & 100 & $\mathrm{NO}$ \\
\hline $\begin{array}{l}\text { MP } \\
(17: 4: 1)\end{array}$ & $\mathrm{NO}$ & 100 & $\mathrm{NO}$ & $<50$ & 100 & 100 & $<50$ \\
\hline MP (1:0) & 88 & 91 & $\mathrm{NO}$ & $<50$ & 97 & 100 & $\mathrm{NO}$ \\
\hline
\end{tabular}


FIG.1

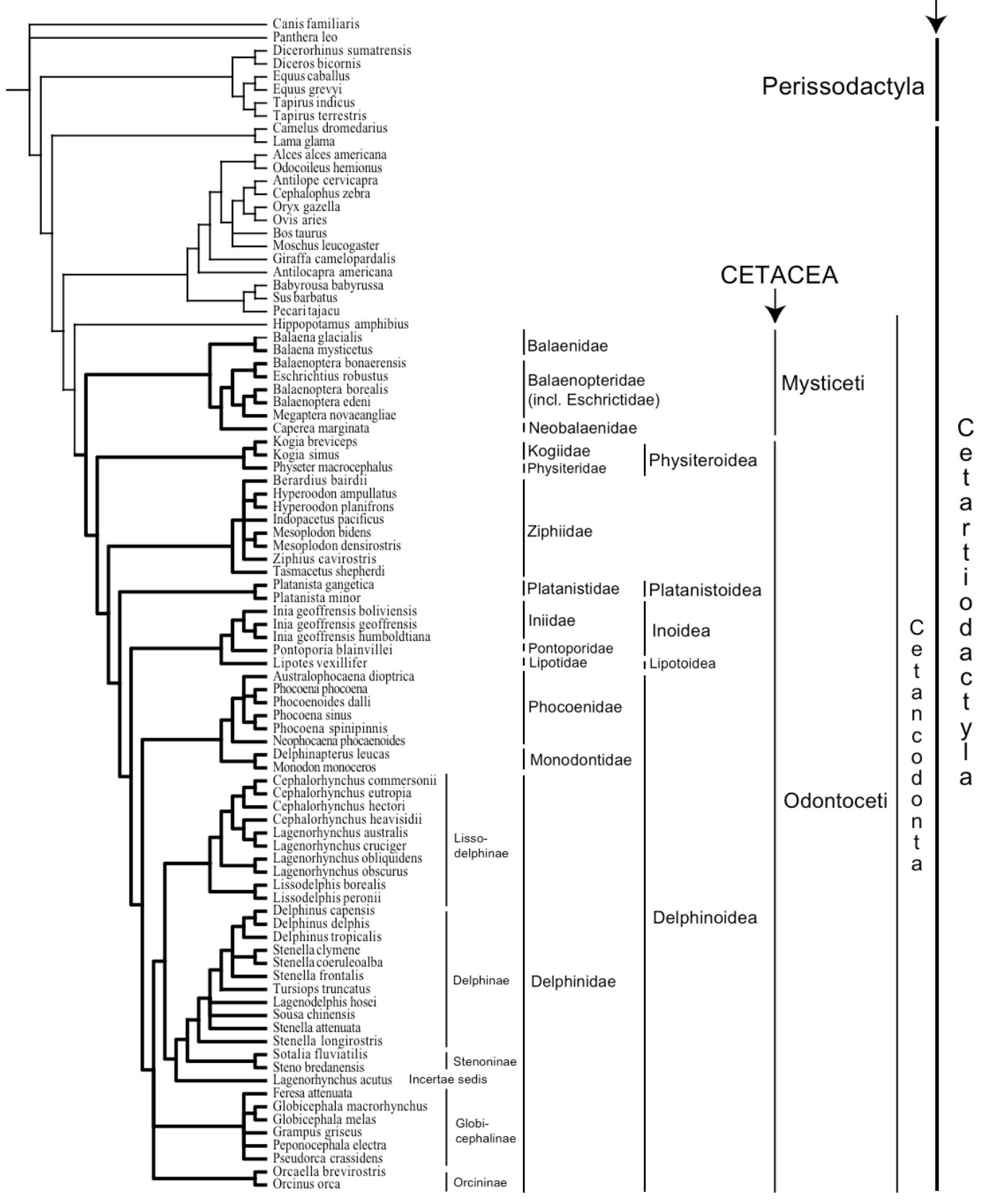


FIG. 2

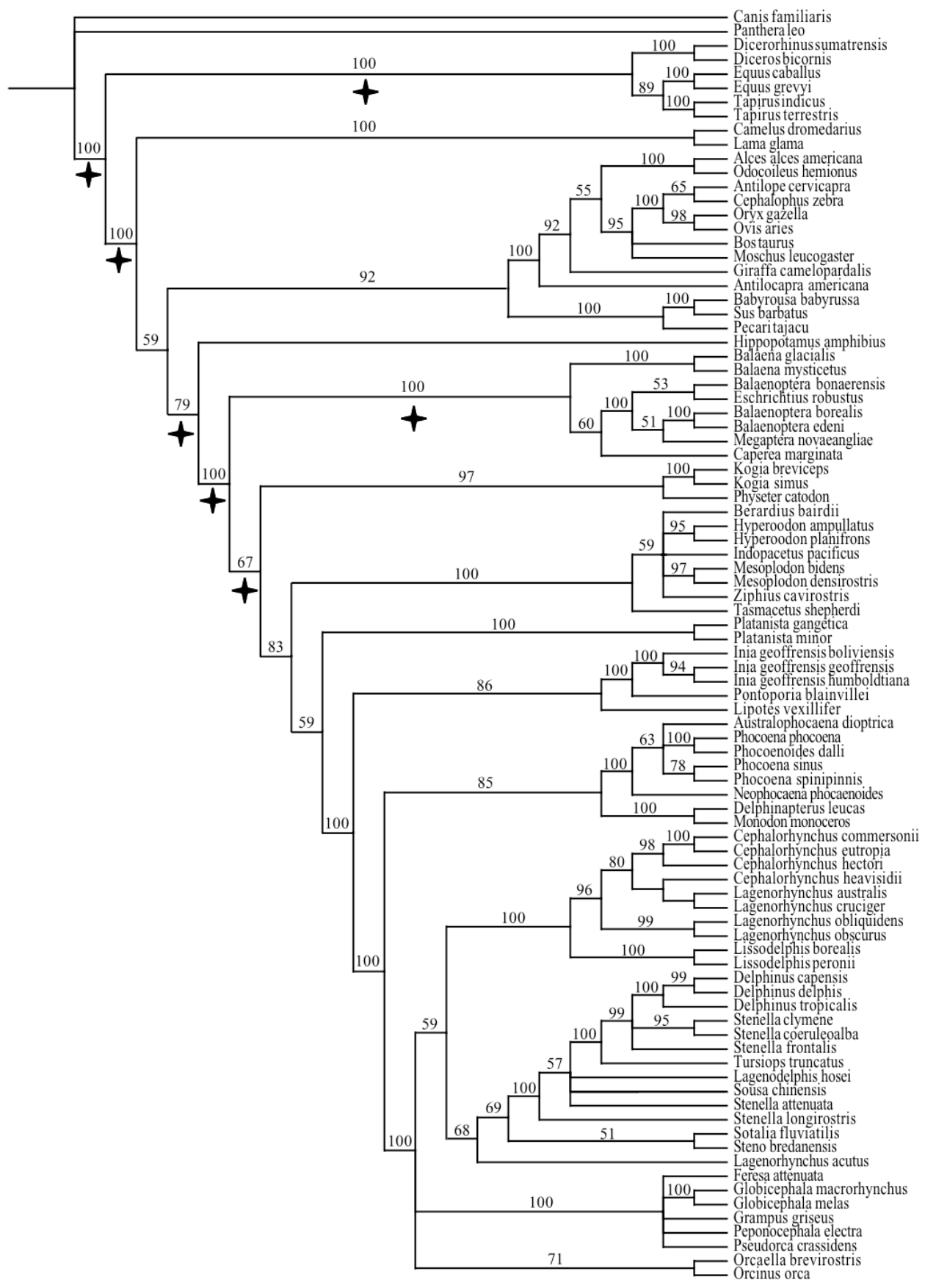




\title{
CHAPTER III
}

\section{THE FRESHWATER DOLPHIN INIA GEOFFRENSIS GEOFFRENSIS PRODUCES HIGH FREQUENCY WHISTLES}

\author{
LAURA J. MAY-COLLADO ${ }^{1,2}$, DOUGLAS WARTZOK ${ }^{1}$ \\ ${ }^{1}$ Florida International University, Department of Biological Sciences, $11200 \mathrm{SW} 8^{\text {th }}$ \\ Street, Miami Florida 33199 \\ ${ }^{2}$ Universidad de Costa Rica, Escuela de Biología, Apto. 2060 San Pedro, Costa Rica
}

May-Collado, L. J. and D. Wartzok. 2007. The freshwater dolphin Inia geoffrensis geoffrensis produces high frequency whistles. Journal of the Acoustical Society of America. 121 (2): 1203-1212. 


\begin{abstract}
Because whistles are most commonly associated with social delphinids, they have been largely overlooked, ignored, or presumed absent, in solitary freshwater dolphin species. Whistle production in the freshwater dolphin, the boto (Inia geoffrensis geoffrensis), has been controversial. Because of its sympatry with tucuxi dolphins (Sotalia fluviatilis), a whistling species, some presume tucuxi whistles might have been erroneously assigned to the boto. Using a broadband recording system we recorded over 100 whistles from boto dolphins in the Yasuní River, Ecuador where the tucuxi dolphins are absent. Our results therefore provide conclusive evidence for whistle production in Inia geoffrensis geoffrensis. Furthermore, boto whistles are significantly different from tucuxi whistles recorded in nearby rivers. The Ecuadorian boto whistle with a significantly greater frequency range (5.30 to $48.10 \mathrm{kHz}$ ) than previously reported in other populations (Peru and Colombia) that were recorded with more bandwidth limited equipment. In addition the top frequency and the range are greater than in any other toothed whale species recorded to date. Whistles production was higher during resting activities alone or in the presence of other animals. The confirmation of whistles in the boto has important implications for the evolution of whistles in Cetacea and their association with sociality.
\end{abstract}




\section{INTRODUCTION}

The Amazon River dolphin, or boto, is known to produce a variety of sounds e.g., echolocation clicks, single intense clicks, jaw snaps, and burst-pulsed sounds (Caldwell and Caldwell 1967, Caldwell et al. 1966). Whistles were first reported by Nakasai and Takemura (1975) in Peru and later documented in more detail by Wang et al. (1995a, 2001). Whistles have been also reported in the boto from the Orinoco River (Diazgranados and Trujillo 2002). Despite these reports, whistle production in this riverine dolphin has been questioned on the basis that boto distribution overlaps largely with tucuxi dolphins (Sotalia fluviatilis), a well documented whistling species. Hence, tucuxi whistles may have been erroneously assigned to the boto (e.g., Podos et al. 2002). In fact, it has been suggested that whistles are unique to social delphinids (Herman and Tavolga 1980, Podos et al. 2002). Certainly, whistles are best studied in social delphinids, where they are used for various communication purposes such as individual identifiers, coordination of group behavior, and maintenance of group cohesion (e.g., Caldwell and Caldwell 1965; Caldwell et al. 1990, Fripp et al. 2005, Herzing 2000, Janik 2000, Tyack 1997, 2000, Watwood et al. 2004). However, non-delphinid toothed whale species like the Chinese river dolphin Lipotes vexillifer (Jing et al. 1981, Xianying et al. 1981,Wang et al. 1989, Wang et al. 2006), the beaked whales of the genus Berardius spp (Dawson et al. 1998, Rogers and Brown 1999), the narwhal Monodon monocerus, and the beluga Delphinapterus leucas (e.g., Belikov and Bel'kovich 2001, 2003, Ford and Fisher 1978, Karlsen et al. 2002, Shapiro 2006, Sjare and Smith 1986, Watkins et al. 1970) are known to produce whistles as well in a variety of contexts. 
Here we document whistles and their behavioral context in the boto dolphins of the Yasuní River, Ecuador and discuss the potential of these signals as communicative signals in this solitary freshwater dolphin. 


\section{MATERIALS AND METHODS}

Study Site

The boto is one of the most widely distributed freshwater dolphins. In parts of its distribution it is sympatric with Sotalia fluviatilis (da Silva 2002). The boto inhabits principal tributaries of the Amazon River as well as small rivers and lakes across its distribution (da Silva 2002). Since one of the main criticisms of previous work on boto whistles is the presence of $S$. fluviatilis in the area of recordings, it was important for our study to be conducted in areas where only botos were found. We selected the Yasuní River a tributary of the Napo River, a narrow river that inundates the adjacent forest and lagoons, during the high-water season (Fig.5). During the low-water season the river becomes narrower and the lagoons dry out (Galacatos et al. 2004), not the type of habitat in which S. fluviatilis is known to occur (da Silva 2002).

Overall $S$. fluviatilis is believed to have low population densities in Ecuador (Dekinger 2001, Zapata-Rios and Utreras 2004). While relatively common in the Putumayo and Morona Rivers, local biologists (Zapata-Rios and Utreras 2004, Victor Utreras pers. comn. 2006), park rangers, and inhabitants of the area confirm that $S$. fluviatilis is rare in the Yasuní, Napo, and Aguarico rivers, and absent in theYasuní during the low-water season. Our study took place in the Yasuní River during the lowwater season when boto were confined to deeper areas of this narrow river.

We recorded boto dolphins from 14 to 19 August 2005 between 6 a.m. to 6 p.m. In order to decrease chances of encountering (and accidentally recording) S. fluviatilis we recorded botos at least $1.5 \mathrm{~km}$ away from the point of intersection with the Napo River 
where the chances to see $S$. fluviatilis may be higher (Zapata-Rios and Utreras 2004). As expected we observed only botos in the Yasuní River and are thus confident that all the whistles presented in this study correspond exclusively to the boto. To verify this, we compare and contrast boto whistles with 20 whistles recorded from six individuals of $S$. fluviatilis and 13 whistles from one group that contained both species at the intersection between the Napo and the Aguarico rivers, about $14 \mathrm{~km}$ downstream from the intersection between Yasuní and the Napo rivers. The 20 whistles recorded from single animals, were very similar in contour, time, and frequency parameters to other $S$. fluviatilis populations (e.g., Podos et al. 2002, Azevedo and Van Sluys 2005, Wang et al. 1995a, 2001). These single animals were recorded near the boat and botos were not present in the area. The other 13 whistles were identical to these in all acoustic parameters therefore they were assigned to S. fluviatilis.

\section{Whistle Definition}

To understand the evolutionary history of whistles and the factors that may have influenced their evolution we must first understand their distribution among cetaceans. To do so it is important not to a priori bias whistle definition e.g. by defining them in the context of a behavior that may have much more limited distribution than the sounds themselves. Rather, it seems less presumptuous to define sounds in terms of acoustic parameters. Whistles are tonal sounds produced by toothed whales. These sounds have a specific acoustic structure that consists of narrowband and frequency modulated sounds (Richardson et al. 1995). Whistles may be complex in contour (e.g., sine, convex, 
concave, upsweep, downsweep) or simply constant in frequency e.g., Lagenorhynchus albirostris (Rasmussen and Miller 2002), Sotalia guianensis (Azevedo and Van Sluys 2005) and Stenella longirostris (Barzúa-Durán and Au 2002). Often whistle fundamental frequency is below $20 \mathrm{kHz}$ (Richardson et al. 1995), but not limited to this range e.g., Oswald et al. (2004) found that Delphinus delphis, Stenella attenuata, S. coeruleoalba, and $S$. longirostris produced whistles with frequencies up to $24 \mathrm{kHz}$, in Lagenorhynchus albirostris whistle fundamental frequency can go up to $35 \mathrm{kHz}$ (Rasmussen and Miller 2002) and up to $41 \mathrm{kHz}$ in Tursiops truncatus (Boisseau 2005). Whistles may be continuous or consist of series of breaks and segments (Richardson et al. 1995) and contain or not harmonics (Au 2000). Some dolphin species like S. longirostris (Lammers and $\mathrm{Au}$ 2003) and L. albirostris (Rasmussen et al. 2006) produce whistles with high order-harmonics. Finally whistles vary greatly in duration. For instance, Sousa chinensis whistles can range from 0.01 to 1.3 seconds (Van Parijs and Corkeron 2001) and in Tursiops truncatus from 0.05 to 3.2 seconds (Wang et al. 1995b).

\section{Recordings and Behavioral observations}

Dolphin signals were recorded using a broadband system consisting of a RESON hydrophone (-203 $\mathrm{dB}$ re $1 \mathrm{~V} / \mu \mathrm{Pa}, 1 \mathrm{~Hz}$ to $140 \mathrm{kHz})$ connected to AVISOFT recorder and Ultra Sound Gate 116 (sampling rate $400-500 \mathrm{kHz} 16$ bit) that sent the signals to a laptop. Recordings were made continuously. For accompanying behavioral observations, recording sessions were segmented into three-minute intervals. Behavioral observations were made in every other three-minute interval and the predominant behavior during that 
interval was recorded. Because the river was narrow and relatively shallow, animals were in sight for the observers most of the time. When animals were not in sight during a 3-min scanning period, the behavior was noted as unknown. Only 3-min periods with acoustic and behavioral information were used for the analyses. Five behavioral categories were defined: (1) Feeding/Foraging, animals that were actively searching, pursuing, and/or consuming prey were assigned to this category, (2) Social activities, when dolphins interacted among themselves e.g., body contact, tail slapping, and animals following the boat or other animals, (3) Traveling, when dolphins were swimming either slowly or fast while maintaining a defined direction, (4) Resting was defined as in Dekinger (2001) were animals showed non directional swimming and surfaced regularly at a slow speed or when surfacing occurred in the same area without any abrupt or fast movement, (5) Unknown behavior, was assigned when the animals were not in sight and thus the behavior activity could not be determined.

Group size, group composition, photo-ID, and geographical position data were also collected. Recordings were obtained from 14 to 19 August 2005, giving a total of 214 files recorded ( $\sim 9$ hours and 45 minutes of recorded time). We analyzed all good quality whistles using the program Raven 1.1 (Cornell Laboratory of Ornithology, New York) with a FFT size of 1024 points, an overlap of 50\%, and using a 512-522 sample Hann window.

Eight parameters were measured for each whistle: starting frequency (SF), ending frequency (EF), minimum frequency (MinF), maximum frequency (MaxF), delta frequency $(\mathrm{DF}=\mathrm{MaxF}-\mathrm{MinF})$, peak frequency $(\mathrm{PF}$, measured in the whistle contour 
were intensity was the highest), duration (s), number of inflection points and contour type. Contour type was categorized as by Azevedo and Simão (2002).

We used SYSTAT ${ }^{\circledR}$ statistical software for descriptive and non-parametric statistics. After testing for normality using the K-S Lilliefors, Skewness, and Kurtosis Tests, non-parametric tests were selected to analyze the data. The Kruskal-Wallis test was used to determine if whistle acoustic parameters varied across behavioral states and groups [sightings] and Chi-square one sample test for Goodness of Fit to determine if whistle production rate (\#whistles/min/individual) varied across behaviors. The Kolmogorov-Smirnov test for two independent samples was used to determine if the medians of the acoustical parameters differed between the two species. A multivariate Discriminant Function Analysis was used to classify whistles within and between species. The Jackknife method was used to calculate the percent of correct classification for each species. 


\section{RESULTS}

A total of 121 high quality tonal sounds fitted the definition of 'whistles' (see above) until now only described in delphinids and a few other toothed whales. However, these whistles were not produced in bouts as in many delphinids species. They were produced singly and spaced in time. The overall whistle production was 0.015 whistles per minute per individual.

Whistle fundamental frequency ranged from 5.30 up to $48.10 \mathrm{kHz}$ and were short in duration (0.002-0.080 sec) (Table 3, Fig.6). About half $(48 \%$; $=58)$ of the whistles had maximum frequency values above $24 \mathrm{kHz}$. This demarcation point was chosen because most dolphin whistles reportedly do not go beyond $24 \mathrm{kHz}$ (with the exceptions mentioned earlier) and this is often the upper limit of recording equipment used in many earlier studies. Similarly $42.1 \%(n=51)$ of beginning frequency, $13.2 \%(n=16)$ of peak frequency, $5.78 \%(n=7)$ of end frequency, and $1.65 \%(n=2)$ of minimum frequency measurements were above $24 \mathrm{kHz}$.

In terms of whistles contours $95.8 \%(n=116)$ of the whistles were descending in frequency. Examples of whistle contours produced by botos are shown in Figure 2 in conjunction with tucuxi whistles for comparison purposes. Only five of all selected whistles had harmonics. The highest frequency harmonic reached $43.5 \mathrm{kHz}$.

The whistles were recorded during three behavioral categories: slow traveling, feeding, and resting. Although more whistles were produced during travel activities when accounting for time and number of individuals, whistle production was significantly higher during resting activities with 0.24 whistles per minute per individual $\left(\chi^{2}=0.50\right.$, $\mathrm{df}=1, \mathrm{p}<0.05)$ compared to traveling $(0.03)$ and feeding $(0.03)$. There were no significant 
differences in the acoustic parameters of whistles across behaviors at p-value 0.05 level (Table 4).

Whistles did vary significantly in their acoustic structure across sighted groups (only groups with more than five whistles were compared) for all whistle parameters except delta frequency (Kruskal-Wallis test, $\mathrm{df}=8, \mathrm{n}=121: \operatorname{MinF} \chi^{2}=20.31, \mathrm{p}=0.026$; $\operatorname{MaxF} \chi^{2}=25.46, p=0.005 ;$ SF $\chi^{2}=23.31, p=0.010 ;$ EF $\chi^{2}=23.86, p=0.008 ;$ PF $\chi^{2}=23.28$, $\mathrm{p}=0.010$; Duration $\chi^{2}=25.46, \mathrm{p}=0.005, \mathrm{DF} \mathrm{p}>0.05$, Table 5). Whistle acoustic parameters did not vary significantly when comparing whistles of groups consisting of adults with groups of adults with calves ( $\mathrm{p}>0.05)$.

When comparing boto and tucuxi whistles we found significant differences in all whistle parameters medians $(\operatorname{MinF}$ Kolmogorov Smirnov $(\mathrm{KS})=0.543, \mathrm{p}<0.001$; MaxF $\mathrm{KS}=0.537, \mathrm{p}<0.001 ; \mathrm{DF} \mathrm{KS}=0.190, \mathrm{p}<0.001, \mathrm{SF} \mathrm{KS}=0.683, \mathrm{p}<0.001 ; \mathrm{EF} \mathrm{KS}=0.298$, $\mathrm{p}<0.001 ; \mathrm{PF} K S=0.336, \mathrm{p}<0.001$; Duration $\mathrm{KS}=0.901, \mathrm{p}<0.001$ ). The boto whistles were higher in frequency for all frequency parameters and much shorter in duration than the whistles produced by tucuxis recorded from the Napo and Aguarico rivers (Fig. 3). Tucuxis produced two classes of whistles that can be described as (1) whistles with maximum frequencies below $20 \mathrm{kHz}$ and minimum frequencies below $10 \mathrm{kHz}$, and (2) whistles with maximum frequencies below $25 \mathrm{kHz}$ and minimum frequencies above 10 kHz (see Fig. 4). Both categories of whistles overlap with boto whistles. Despite of this overlap in frequency, the discriminant function analysis correctly classified (based on all acoustical parameters) all boto whistles (100\%). Only $15 \%$ of the tucuxi whistles were incorrectly classified. 


\section{DISCUSSION}

Our results confirm previous findings that botos indeed whistle (Wang et al. 1995, 2001, Diazgranados and Trujillo 2002) and suggest that boto whistles are frequency modulated with one of the widest frequency ranges ever reported in a toothed-whale species.

Interestingly, the acoustic structure of these whistles is not only distinct from those of the sympatric $S$. fluviatilis but also appears quite distinct from other acoustically known boto populations in Colombia (Inia geoffrensis humboldtiana) (Diazgranados and Trujillo 2002) and Perú (Inia geoffrensis geoffrensis) (Wang et al. 1995a, 2001). However, the comparison between these studies is difficult due to differences in recording equipment as discussed below.

\section{Between and within species variation}

Boto whistles differ from tucuxi whistles in all their acoustical parameters. As shown in Figure 4, botos produced higher frequency whistles. These whistles are more limited in their contour diversity (95.8\% of the whistles were downsweep) than tucuxi whistles (and those of most other dolphins). Several factors have been proposed to explain interspecific whistle variation including: morphological constraints (Wang et al. 1995a, Matthews et al. 1999), environment (Wang et al. 1995a), sociality (Podos et al. 2002), zoogeographical relationships (Steiner 1981), and phylogenetic relationships (Steiner 1981, Wang et al. 1995a).

Body size is the most important morphological factor believed to influence signal frequency in animals (Marquet and Taper 1998). Overall, the larger the animal the lower frequency sounds it tends to produce. This is because body size and the size of sound 
producing organs are often correlated (Fletcher 1992). Some authors (e.g., Wang et al. 1995a, Podos et al. 2002) have proposed a similar relationship between body size and maximum frequency. However, in the case of cetaceans a recent study showed that when accounting for phylogeny the proposed relationship between body size and maximum frequency disappears (May-Collado et al. 2007a). Thus the fact that botos ( 2.6 m, 160 $\mathrm{kg})$ can produce much higher frequency whistles than the smaller tucuxis $(\sim 1.52 \mathrm{~m}, 40$ $\mathrm{kg}$ ) is not counter to any general rule.

It is unlikely that whistle variation is explained by differences in habitat acoustic structure since both species live in very similar environmental conditions. Another proposed factor to explain interspecific whistle variation is zoogeographical relations. Steiner (1981) suggested "the degree of differences in the whistle vocalizations among [five dolphin] species closely followed predictions based on classic allopatric/sympatric relations among species". This idea is congruent with the "species recognition hypothesis" (see Sætre et al. 1997) that states that animal vocal acoustic structure has evolved 'to' reduce hybridization. Unfortunately, there is very little quantitative information of the extent to which botos and tucuxis are allopatric and sympatric at both spatial and temporal scales to test this idea.

Botos and tucuxis are not closely related (e.g., Hamilton et al. 2001, May-Collado and Agnarsson 2006) which could largely explain their differences in whistle structure. However, there is recent evidence that social structure (or at least some components of sociality) could also explain part of this variation. For instance, differences in whistle contour and frequency and time parameters of the distantly related spinner and bottlenose dolphins (e.g., Hamilton et al. 2001, LeDuc et al. 1999, May-Collado and Agnarsson 
2006) have been largely explained in terms of "group fluidity" [a component of sociality] (Barzúa-Durán 2004). Botos and tucuxis are not only more distantly related but also differ even more radically in their social structure. While botos appear to be solitary (Best and da Silva 1993) or at least live in small non-structured groups (where the strongest social bond appear to be limited to mother and calf) (e.g., Aliaga-Rossel 2002, McGuire and Winemiller 1998), Sotalia spp. lives in structured social groups (MonteiroFilho 2000). Additionally, May-Collado et al. 2007b found that whistle complexity-a concept based on whistle mean number of inflection points - may be influenced by group size and social structure [two components of sociality]. More specifically, they found that simple whistles (mean number of inflection points equal or below one) were particularly concentrated in 'solitary' species while the phylogenetic distribution of complex whistles and social species largely overlap.

Although boto dolphins from the Yasuní River produced whistles with frequency parameters that appear to be far above (5.30 to $48.10 \mathrm{kHz})$ the values reported by Wang et al. (1995a, 2001) in botos from the Marañon and Tigre Rivers in Peru (0.22 to 5.16 $\mathrm{kHz}$ ) and by Diazgranados and Trujillo (2002) in the Orinoco River (3 to $13 \mathrm{kHz}$ ) (Table 3) comparisons can not be done at this point. This is due in part to differences in the recording systems maximum frequency limitations (up to $25 \mathrm{kHz}$ ), to the lack of information on several standard acoustic parameters (Diazgranados and Trujillo (2002), and the uncertainty regarding the assignment of the low frequency whistles to boto dolphins (Wang et al. 1995a, 2001). Therefore, until we have full frequency range recordings from other botos populations, comparisons are difficult and speculative. 
We limit this part of the discussion to the observed differences among 'groups' groups [note that several of the 'groups' consisted of only one individual] where with the exception for delta frequency, whistle acoustic parameters vary significantly across groups (Table 5). In most delphinids within species variation is primarily in duration and modulation (e.g., Wang et al. 1995b, Morisaka et al. 2005a-b, Barzúa-Dúran and Au 2002,2004 ) rather than in frequency as observed in this boto population (also see Azevedo and Van Sluys 2005, Rossi-Santos and Podos 2006 for Sotalia guianensis). In part, this variation has been explained as product of adaptation to local ecological conditions (e.g., ambient noise, see Morisaka et al. 2005b). It is unlikely that the among group whistle differences observed in this boto population are explained by contrasting habitat acoustic characteristics alone, since recorded single animals and groups were in the same environment. Furthermore, age composition and behavioral states appear not to influence whistle variation either. However, our sample size is too small and geographically restricted to conclude age and behavior does not have some influence on boto dolphin whistles acoustic structure. In addition to these two factors, others such as genes, gender, and overall inter-individual variation, merit further study.

\section{Behavior and Communication}

Diazgranados and Trujillo (2002) reported that boto whistles were produced within groups that engaged in social and feeding activities. Half of the whistles produced by the botos in this study occurred during traveling activities, but when accounting for time of the encounter and number of individuals present, whistles production was higher during 
resting activities. In addition, whistles were produced by both solitary and grouped animals.

The closest neighbor maximum distance was found at approximately 0.15 to 1.5 $\mathrm{km}$, suggesting these animals might be still in acoustic contact. Because of the behavioral context at which most whistles were emitted and the low whistle production, it is possible that whistles in boto dolphins may be used to keep distance between animals, rather than promote social interactions or cohesion among individuals as it occurs in delphinids (e.g., Jones and Sayigh 2002). Assuming cylindrical spreading loss and freshwater absorption at the mean maximum frequency, the boto whistles could propagate as far as $3.3 \mathrm{~km}$ before falling below the ambient noise although the actual range will be less depending on channel depth, bottom type, and vegetation (Quitana-Rizzo et al. 2006). Wang et al. (2006) assuming spherical spreading losses estimated that Lipotes vexillifer low frequency whistles (with dominant frequency $5.7 \mathrm{kHz}$ ) could propagate in a very calm environment up to $6.6 \mathrm{~km}$, but possibly only $22-220 \mathrm{~m}$ considering the noisy conditions of the Yangtzee River. The Yasuní River is a very calm environment, unlike the Yangtzee River, it is protected and boat traffic is limited to park rangers and scientists.

During the period of this study botos produced whistles randomly and not in bouts as many delphinids species. Based on our data it appears that boto dolphins in Yasuní whistle rarely. Our sample size is too small not only to clearly associate these sounds with the same social contexts as in delphinids but also to determine how frequently botos and other riverine dolphin species generally whistle. Nevertheless, confirming the presence of whistles in botos and other freshwater toothed whales helps illuminate the 
evolutionary history of whistles, and their relation to sociality - a factor proposed to have shaped the complexity of toothed whale whistles (May-Collado et al. 2007b). 


\section{CONCLUDING REMARKS}

This study confirms whistles in the solitary freshwater dolphin Inia geoffrensis geoffrensis. The frequency range of boto whistles we document is among the greatest ever reported in a cetacean species. The acoustic structure of these whistles in Ecuadorian botos differs from that of the sympatric Sotalia fluviatilis and apparently from conspecific populations from Perú and Colombia. Differences in the acoustic structure of boto and tucuxi whistles may be in large a product of their distant phylogenetic relationships and their different social structure. Although, our study suggests remarkable whistle variation between populations this may be largely a product of limited recording systems used by previous studies. Finally, although the use of whistles for communication purposes has been largely attributed to social dolphin species, it is possible that botos (even solitary animals) use them to communicate but in the context of keeping distances among animals rather than to promote group cohesion. We propose this based on two observations (1) whistles were produced when the animals were engaged in resting activities, and (2) based on the fact that solitary animals were potentially within acoustic range of each other (estimated maximum of $3.3 \mathrm{~km}$ ). Finally, better understanding of whistle production in solitary freshwater species will further understanding of the evolutionary history of whistles and their proposed association with sociality. 


\section{ACKNOWLEDGEMENTS}

We thank Ingi Agnarsson, Universtiy of British Columbia, Tim Collins, Mike Heithaus, Zhenim Chen, Florida International University and two anonymous reviewers for comments and suggestions to this manuscript. Thanks to Maureen Donnelly for her support. Thanks to Alonso Jaramillo Encargado del Parque Nacional Yasuní, Ecuador, Dr. Luis Albuja del Departamento de Ciencias Biologicas de la Escuela Politécnica Nacional, the boat captains Walter Prado and Daniel Contreras, Biologists Víctor Utreras and Judith Denkinger, field assistants Mónica Gamboa-Poveda and Angélica Caiza. Locals Don Segundo, Estuardo Hidalgo, and all the park rangers at Yasuní National Park. Recordings were carried out with permission of the Ministerio del Ambiente de la República de Ecuador and the National Park Yasuní (Permit No. 019-IC-FL-PNY-RSO) and authorization from the IACUC committee at Florida International University. This study was fund by Judith Parker Travel Grant, Cetacean International Society, and the Russell E. Train Education Program-WWF to Laura May-Collado. 


\section{LIST OF REFERENCES}

Aliaga-Rossel, E. (2002). Distribution and abundance of the river dolphin (Inia geoffrensis) in the Tijamuchi River, Beni, Bolivia. Aq. Mamm. 28, 312-323.

Azevedo, A. F., and Van Sluys, M. (2005). "Whistles of tucuxi dolphins (Sotalia fluviatilis) in Brazil: comparisons among populations". J. Acoust. Soc. Am. 117, 14561464.

Azevedo, A. F., and Simão, S. M. (2002). "Whistles produced by marine tucuxi dolphins Sotalia fluviatilis in Guanabara Bay, southeastern Brazil". Aquat. Mamm. 28, 261-266.

Au, W. W. L. (2000). "Hearing in whales and dolphins: An overview" in Hearing by Whales and Dolphins edited by Au W. W. L., Popper, A. N., and R. R., Fay (SpringerVerlag Press, New York). Pp.1-42.

Barzúa-Durán, M. C., and Au, W. W. L. (2002). "Whistles of Hawaiian spinner dolphins". J. Acoust. Soc. Am. 112, 3064-3072.

Barzúa-Durán, M. C. (2004). Differences in the whistle characteristics and repertoire of bottlenose and spinner dolphins. An. Acad. Bras. Cienc. 76, 386-392.

Barzúa-Durán, M. C. and Au, W. W. L. (2004). "Geographic variations in the whistles of spinner dolphins (Stenella longirostris) of the Main Hawaiian Islands". J. Acoust. Soc.

Am. 116, 3757-3769.

Belikov, R. A., and Bel'kovich, V. M. (2001). "Characteristics of white sea beluga whale (Delphinapterus leucas) whistle-like signals". XI Session of the Russian Acoustical Society, Moscow, November 19-23.

Belikov, R. A., and Bel'kovich, V. M. (2003). "Underwater vocalization of the beluga whales (Delphinapterus leucas) in a reproductive gathering in various behavioral situations". Oecologia. 43, 112-120.

Best, R. C., and da Silva, V. M. F. (1993). "Inia geoffrensis". Mammalian Species. 426, $1-8$.

Boisseau, O. (2005). "Quantifying the acoustic repertoire of a population: the vocalizations of free-ranging bottlenose dolphins in Fiordland, New Zealand". J. Acoust. Soc. Am. 117, 2318-2329.

Caldwell, M.C., and Caldwell, D.K. (1965). "Individual whistle contours in bottlenose dolphins (Tursiops truncatus)". Nature (Lond.). 207, 434-435. 
Caldwell, M. C., and Caldwell, D. K. (1967). "Intra-specific transfer of information via the pulsed sound in captive odontocete cetaceans,' in Animal Sonar Systems, edited by R.-G. Busnel (Jouy-en-Josas, France). 2, 879-936.

Caldwell, M. C., Caldwell, D. K., and Evans, W. E. (1966). 'Sounds and behavior of captive Amazon freshwater dolphin, Inia geoffrensis,' Los Angeles County Mus. Cont. Sci. 108, 1-24.

Caldwell, M. C., Caldwell, D. K., and Tyack, P. L. (1990). "Review of the signaturewhistle hypothesis for the Atlantic bottlenose dolphin" in The bottlenose dolphin edited by Leatherwood, S. and R. R. Reeves (Academic Press, San Diego). Pp. 199-234.

da Silva, V. M. F. (2002). "Amazon River Dolphin (Inia geoffrensis)" in Encyclopedia of Marine Mammals, edited by W. F. Perrin, B. Wursig, and J. G. M. Thewissen (Academic Press, San Diego), pp. 18-20.

Dawson, S., Barlow, J., and Ljungblad D. (1998). "Sounds recorded from Baird's beaked whale, Berardius bairdii”. Mar. Mamm. Sci. 14, 335-344.

Dekinger, J. (2001). "Demographische Untersuchunge am Amazonasdelphin (Inia geoffrensis) in Cuyabeno Reseervat, in Ecuador". Ph. D. Dissertation. Universitat Bielefeld, Bielefeld Germanay.

Diazgranados, M. C., and Trujillo, F. (2002). "Vocal repertoire of the freshwater dolphins Inia geoffrensis and Sotalia fluviatilis in Colombia, South America". J. Acoust. Soc. Am. 112, 2400.

Fletcher, N. H. (1992). “Acoustic Systems in Biology”. Oxford University Press, Oxford, England. 352 pp.

Ford, J. K. B., and Fisher, H. D. (1978). "Underwater acoustic signals of the narwhal (Monodon monocerus)”. Can. J. Zool. 56, 552-560.

Fripp D., Owen C., Quintana-Rizzo E., Shapiro A., Buckstaff K., Jankowski K., Wells R., and Tyack, P. (2005). "Bottlenose dolphin (Tursiops truncatus) calves appear to model their signature whistles on the signature whistles of community members". Anim. Cogn. 8, 17-26.

Galacatos, K., Barriga-Salazar, R., and Steward, D. J. (2004). "Seasonal and habitat influences on fish communities within the lower Yasuní River Basin of the Ecuatorian Amazon". Environ. Biol. Fishes. 71, 33-51.

Hamilton, H., Caballeron, S., Collins, A. G., and Brownell Jr. R. L. (2001). "Evolution of river dolphins". Proc. Royal Soc. Lond. B. 268, 549-558. 
Herman, L. M., and Tavolga, W. N. (1980). "The communication systems of cetaceans" in Cetacean Behavior: Mechanisms and Functions, edited by Herman-L. M. (John Wiley \& Sons, New York).

Herzing, D. L. (2000). "Acoustics and social behavior of wild dolphins: implications for a sound society" in Hearing by whales and dolphins edited by Au W. W. L., Popper, A. N. and R. E. Fay (Springer Press, New York), pp. 225-272.

Janik, V. M. (2000). "Whistle matching in wild bottlenose dolphins (Tursiops truncatus)”. Science. 289, 1355-1357.

Jing, X., Xiao, Y., and Jing, R. (1981). "Acoustic signals and acoustic behavior of Chinese river dolphin (Lipotes vexillifer).” Scient. Sinica. 2, 233-239.

Jones, G. J., and Sayigh, L. S. (2002). "Geographic variation in rates of vocal production of free-ranging bottlenose dolphins”. Mar. Mamm. Sci. 18, 384-393.

Karlsen, J.D., Bisther A, Lydersen, C., Haug, T., and Kovacs, K. M. (2002). "Summer vocalisations of adult male white whales (Delphinapterus leucas) in Svalbard, Norway". Polar Biol. 25, 808-817.

Lammers, M. O., and Au, W. W. L. (2003). "Directionality in the whistles of Hawaiian spinner dolphins (Stenella longirostris): a signal feature to cue direction of movement?" Mar. Mamm. Sci. 19, 249-264.

LeDuc, R.G., Perrin, W.F., and Dizon, A.E. (1999). "Phylogenetic relationships among the delphinid cetaceans based on full cytochrome $b$ sequences". Mar. Mamm. Sci. 15, 619-648.

Marquet, P.O., and M.L. Taper. (1998). "On size and area: patterns of mammalian body size extreme across landmasses”. Evol. Bio. 12,127-139.

Matthews, J.N., Rendell, L.E., Gordon, J.C.D., and MacDonald, D.W. (1999). “A review of frequency and time parameters of cetacean tonal calls". Bioacoustics 10, 47-71. 21

May-Collado, L. J. and I. Agnarsson. (2006). "Cytochrome b and Bayesian inference of whale phylogeny”. Molec. Phylogen. and Evol. 38, 344-354.

May-Collado, L. J., Agnarsson, I., and Wartzok, D. (2007a) Reexamining the relationship between body size and tonal signals frequency in whales: a comparative phylogenetic approach. Mar. Mamm Sci. 23(3): 524-552.

May-Collado, L. J., Agnarsson, I., and D. Wartzok. (2007b). "Phylogenetic review of tonal sound production in whales in relation to sociality". BMC Evol. Bio. 7(136). 
McGuire, T. L., and Winemiller, K. O. (1998). "Ocurrence patterns, habitat associations, and potential prey of the river dolphin, Inia geoffrensis, in the Cinaruco River, Venezuela". Biotropica. 30, 625-638.

Monteiro-Filho, E. L. A. (2000). "Group organization of the dolphin Sotalia fluviatilis guianensis in an estuary of southeastern Brazil". Ciencia e Cultura J. Braz. Ass. Adv. Sci. $\mathbf{5 2}, 97-101$.

Morisaka, T., Shinohara, M., Nakahara, F., and Akamatsu, T. (2005a). “Geographic variations in the whistles among three Indo-Pacific bottlenose dolphin Tursiops aduncus populations in Japan”. Fish. Sci. 71, 568-576.

Morisaka, T., Shinohara, M., Nakahara, F. and Akamatsu, T. (2005b). "Effects of ambient noise in the whistles of Indo-Pacific bottlenose dolphin Tursiops aduncus populations in Japan”. J. Mamm. 86, 541-546.

Nakasai, K., and Takemura, A. (1975). "Studies on the underwater sound. VI. On the underwater calls of freshwater dolphins in South America,', Bull. Fac. Fish. Nagasaki Univ. 40, 7-13.

Oswald J. N., Rankin, S., and Barlow, J. (2004). "The effect of recording and analysis bandwidth on acoustic identification of delphinid species". J. Acoust. Soc. Am. 116, 3178-3185.

Podos, J., da Silva, V. M. F., and Rossi-Santos, M. R. (2002). "Vocalizations of Amazon river dolphins, Inia geoffrensis: insights into the Evolutionary origins of delphinid whistles”. Ethology 108, 601-612.

Quintana-Rizzo, E., Mann, D.A. and Wells, R.S. (2006). "Estimated communication range of social sounds used by bottlenose dolphins (Tursiops truncatus)". J. Acoust. Soc. Am. 120, 1671-1683.

Rasmussen, M. H., and Miller, L. A. (2002). "Whistles and clicks from white-beaked dolphins, (Lagenorhynchus albirostris Gray 1846) recorded in Faxafloi Bay, Iceland".Aquatic Mamm. 28, 78-89.

Rasmussen, M. H., Lammers, M., Beedholm, K., and Miller, L. A. (2006). "Source levels and harmonic content of whistles in white-beaked dolphins (Lagenorhynchus albirostris)". J. Acoust. Soc. Am. 120, 511-517.

Richardson, W. J., Greene, C. R. J., Malme, C. I., and Thomsom, D. H. (1995). "Marine Mammals and Noise" (Academic, New York). 547 pp.

Rogers, T. L., and Brown, S. M. (1999). "Acoustic observations of Arnoux's beaked whale (Berardius arnuxii) off Kemp Land, Antarctica”. Mar. Mamm. Sci. 15, 192-198 
Rossi-Santos, M. R., and Podos, J. (2006). "Latitudinal variation in whistle structure of the estuarine dolphin Sotalia guianensis". Behaviour. 143, 347-364.

Sætre, G. P., T. Moum, S. Bures, M. Kral, M. Adamjan, and Moreno, J. (1997). “A sexually selected character displacement in flycatchers reinforces premating isolation". Nature. 387, 589-591.

Shapiro, A. (2006). Preliminary evidence for signature vocalizations among free-ranging narwhals (Monodon monocerus). J. Acoust. Soc. Am. 120, 1695-1705.

Sjare, B. L., and Smith, T. G. (1986). "The vocal repertoire of white whales, Delphinapterus leucas, summering in Cunningham Inlet, Northwest Territories". Can J. Zool. 64, 407-415.

Steiner, W. W. (1981). "Species-specific differences in pure tonal whistle vocalizations of five western North Atlantic dolphin species". Behav. Ecol. Soc. 9, 241-246.

Tyack, P. L. (1997) "Development and social functions of signature whistles in bottlenose dolphins Tursiops truncatus”. Bioacoustics 8, 21-46

Tyack, P. L. (2000). "Functional aspects of cetacean communication" in Cetacean Societies: Field studies of dolphins and whales edited by Mann, J., Connor, R. C., Tyack, P. L., and H. Whitehead (The University of Chicago Press, Chicago), pp. 270-307.

Van Parijs, S. M. and Corkeron, P. J. (2001). "Vocalizations and behaviour of Pacific Humbpaback dolphins, Sousa chinensis”. Ethology. 107, 701-716.

Wang, D., Lu, W., and Wang, K. (1989). “A preliminary study of the acoustic behavior of the baiji, Lipotes vexillifer,', Occasional Papers of the IUCN Species Survival Commission (SSC) No. 3, pp. 137-140.

Wang, D., Würsig, B., and Evans, W. E. (1995a). "Comparisons of whistles among seven odontocete species" in Sensory Systems of Aquatic Mammals edited by. R. A. Kastelien, J. A. Thomas, and P. E. Nachtigal. De Spill Publishers, Woerden, The Netherlands, Pp. 299-323.

Wang, D., Wursig, B., and Evans, W. E. (1995b). "Whistles of bottlenose dolphins: comparisons among populations". Aquatic Mamm. 21, 65-77.

Wang, D., Würsig, B., and Leatherwwod, S. (2001). "Whistles of boto, Inia geoffrensis, and tucuxi, Sotalia fluviatilis”. J. Acoust. Soc. Am. 109, 407-414.

Wang, X., Wang, D., Akamatsu, T., Fujita, K. and Shiraki, R. (2006). Estimated detection distance of a baiji's (Chinese river dolphin, Lipotes vexillifer) whistles using a passive acoustic survey method. J. Acoust. Soc. Am. 120, 1361-1365. 
Watkins, W. A., Schevill, W. E., and Ray, C. (1970). "Underwater sounds of Monodon (Narwhal)". J. Acoust. Soc. Am. 49, 595-599.

Watwood S. L., Tyack P. L., and Wells, R. S. (2004). "Whistle sharing in paired male bottlenose dolphins, Tursiops truncatus". Behav. Ecol. Sociobiol. 55, 531-543.

Xianying, J., Youfo, X. and Rongcai, J. (1981). "Acoustic signals and acoustic behavior of the Chinese River Dolphin (Lipotes vexillifer)”. Scient. Sinica. 24:407-415.

Zapata-Ríos, G., and Utreras, V. B. (2004). "Notes on the distribution of tucuxi, Sotalia fluviatilis (Cetacea:Delphinidae), in Ecuadorian Amazonia". Lat. Am. J. Aquatic Mamm. 3, 85-87. 
Table 3. Descriptive statistics of boto whistles with comparison to previously published data (in bold values for all 121 whistles and in parenthesis values for 70 whistles with frequency values below $25 \mathrm{kHz}$ for comparison purposes).

\begin{tabular}{|c|c|c|c|c|c|c|c|}
\hline & $\operatorname{Min} F(k H z)$ & $\operatorname{Max} F(\mathrm{kHz})$ & Start $F(\mathrm{kHz})$ & End $F(\mathrm{kHz})$ & Delta $F(\mathrm{kHz})$ & Peak F $(\mathrm{kHz})$ & Duration (s) \\
\hline \multicolumn{8}{|c|}{ This Study } \\
\hline Mean & $14.54(12.59)$ & $24.71(19.26)$ & $23.30(18.49)$ & $15.48(13.10)$ & $10.18(6.71)$ & $18.62(15.36)$ & $\mathbf{0 . 0 0 9}(0.010)$ \\
\hline$S D$ & $4.32(3.96)$ & $8.37(4.0)$ & $\mathbf{8 . 5 3}(4.10)$ & $\mathbf{5 . 7 0}(4.35)$ & $7.02(2.47)$ & $\mathbf{6 . 6 1}(4.52)$ & $0.011(0.014)$ \\
\hline Range & $5.30-26.44$ & $10.88-48.10$ & $9.77-48.10$ & $5.30-42.99$ & 2.94-34.39 & $3.22-48.83$ & $0.002-0.080$ \\
\hline & $(5.30-21.37)$ & $(10.88-24.89)$ & $(9.77-24.89)$ & $(5.30-24.62)$ & $(2.99-16.52)$ & $(6.35-22.95)$ & $(0.002-0.080)$ \\
\hline $\mathrm{CV} \%$ & $29.7(31.5)$ & $33.9(20.8)$ & $36.1(22.1)$ & $38.60(33.3)$ & $68.9(36.9)$ & $\mathbf{3 5 . 5 0}(29.5)$ & $128.2(134.2)$ \\
\hline \multicolumn{8}{|c|}{ Wang et al. $(1995,2001)^{*}$} \\
\hline Mean & 2.54 & 2.97 & 2.61 & 2.86 & ------------ & ------------- & 1.14 \\
\hline$S D$ & 0.76 & 0.84 & 0.75 & 0.77 & ------------ & ------------ & 1.01 \\
\hline Range & $0.220-4.22$ & $0.5-5.16$ & $0.220-4.22$ & $0.360-4.86$ & ------------- & ------------- & $0.16-4.42$ \\
\hline$C V \%$ & 29.88 & 28.11 & 28.55 & 27.01 & ------------ & ------------- & 91.10 \\
\hline \multicolumn{8}{|c|}{ Diazgranados and Trujillo (2002) } \\
\hline Mean & ------------ & ------------ & ------------ & ------------ & ------------- & ------------- & ------------ \\
\hline$S D$ & ------------ & ------------- & ------------ & ------------ & ------------- & ------------ & ------------- \\
\hline Range & 3 & 13 & ------------ & ------------ & ------------ & ------------ & ------------ \\
\hline$C V \%$ & ------------- & ------------ & ------------- & ------------- & ------------- & ------------- & ------------- \\
\hline
\end{tabular}

*Recording system with maximum frequency limited to $25 \mathrm{kHz}$ 
Table 4. Descriptive statistics for each behavioral class for a total of 121 whistles recorded in relation to their behavioral context (there were not significant differences for any of these parameters across behaviors).

\begin{tabular}{|c|c|c|c|}
\hline Parameters & Feeding $(n=32)$ & Resting $(n=21)$ & Slow Traveling $(n=68)$ \\
\hline $\begin{array}{c}\text { Min F (kHz) } \\
\text { Range } \\
C V \%\end{array}$ & $\begin{array}{l}14.48 \pm 4.95 \\
6.56-21.22 \\
28.0\end{array}$ & $\begin{array}{c}14.59 \pm 4.66 \\
6.26-23.10 \\
32.0\end{array}$ & $\begin{array}{c}14.56 \pm 4.39 \\
5.30-26.44 \\
30.2\end{array}$ \\
\hline $\begin{array}{c}\text { Max F } \mathbf{( k H z )} \\
(\text { mean } \pm S D) \\
\text { Range } \\
C V \% \\
\end{array}$ & $\begin{array}{c}25.25 \pm 9.28 \\
11.83-43.68 \\
36.8 \\
\end{array}$ & $\begin{array}{c}22.84 \pm 6.97 \\
12.76-41.72 \\
30.5 \\
\end{array}$ & $\begin{array}{c}25.03 \pm 8.36 \\
10.88-48.06 \\
33.4 \\
\end{array}$ \\
\hline $\begin{array}{c}\text { Delta F (kHz) } \\
\text { (mean } \pm S D) \\
\text { Range } \\
C V \% \\
\end{array}$ & $\begin{array}{c}10.84 \pm 7.84 \\
3.82-28.24 \\
72.3 \\
\end{array}$ & $\begin{array}{c}8.25 \pm 6.48 \\
2.99-30.35 \\
78.6 \\
\end{array}$ & $\begin{array}{c}10.47 \pm 6.77 \\
2.94-34.40 \\
64.7 \\
\end{array}$ \\
\hline $\begin{array}{c}\text { Start F } \quad(\mathbf{k H z}) \\
(\text { mean } \pm S D) \\
\text { Range } \\
C V \% \\
\end{array}$ & $\begin{array}{c}22.79 \pm 9.06 \\
11.83-43.68 \\
39.7 \\
\end{array}$ & $\begin{array}{c}22.15 \pm 7.14 \\
12.76-41.72 \\
32.2 \\
\end{array}$ & $\begin{array}{c}24.43 \pm 8.68 \\
9.77-48.06 \\
35.5 \\
\end{array}$ \\
\hline $\begin{array}{c}\text { End F } \mathbf{( k H z )} \\
(\text { mean } \pm S D) \\
\text { Range } \\
C V \% \\
\end{array}$ & $\begin{array}{c}16.85 \pm 7.85 \\
7.06-42.98 \\
46.6 \\
\end{array}$ & $\begin{array}{c}15.62 \pm 5 . .21 \\
6.26-26.85 \\
33.3 \\
\end{array}$ & $\begin{array}{c}14.80 \pm 4.50 \\
5.30-27.34 \\
30.4 \\
\end{array}$ \\
\hline $\begin{array}{c}\text { Peak F } \mathbf{( k H z )} \\
(\text { mean } \pm S D) \\
\text { Range } \\
C V \% \\
\end{array}$ & $\begin{array}{c}17.95 \pm 4.95 \\
7.81-27.34 \\
27.6 \\
\end{array}$ & $\begin{array}{c}16.15 \pm 5.80 \\
3.22-26.86 \\
35.9 \\
\end{array}$ & $\begin{array}{c}18.49 \pm 5.75 \\
6.35-33.69 \\
31.1 \\
\end{array}$ \\
\hline $\begin{array}{c}\text { Duration (s) } \\
\text { Range } \\
C V \%\end{array}$ & $\begin{array}{c}0.006 \pm 0.007 \\
0.002-0.039 \\
111.5 \\
\end{array}$ & $\begin{array}{c}0.008 \pm 0.008 \\
0.002-0.039 \\
103.4 \\
\end{array}$ & $\begin{array}{c}0.010 \pm 0.014 \\
0.002-0.080 \\
129.4 \\
\end{array}$ \\
\hline
\end{tabular}


Table 5. Whistle acoustic structure described for each recorded single animal and group.

\begin{tabular}{|c|c|c|c|c|c|c|c|c|c|c|}
\hline Groups & $\begin{array}{r}\operatorname{Min} \mathbf{F} \\
(\mathrm{kHz})\end{array}$ & $\begin{array}{c}\operatorname{Max} F \\
(\mathbf{k H z})\end{array}$ & $\begin{array}{c}\text { Delta F } \\
(\mathbf{k H z})\end{array}$ & $\begin{array}{c}\text { Start F } \\
(\mathbf{k H z})\end{array}$ & $\begin{array}{l}\text { End F } \\
(\mathrm{kHz})\end{array}$ & $\begin{array}{c}\text { Peak F } \\
(\text { kHz) }\end{array}$ & $\begin{array}{l}\text { Duration } \\
\text { (s) }\end{array}$ & $\begin{array}{c}\# \\
\text { whistles }\end{array}$ & $\begin{array}{c}\text { \#Individuals } \\
\text { present }\end{array}$ & $\begin{array}{c}\text { Group } \\
\text { Composition }\end{array}$ \\
\hline G1 Mean $\pm S D$ & $17.20 \pm 2.48$ & $30.23 \pm 8.28$ & $13.04 \pm 7.62$ & $29.63 \pm 9.27$ & $17.80 \pm 2.60$ & $23.43 \pm 4.56$ & $0.006 \pm 0.007$ & 6 & 1 & Adult* \\
\hline Range & $13.90-20.04$ & $19.71-38.60$ & $3.62-23.27$ & $16.10-38.60$ & $13.9-20.04$ & $17.58-27.34$ & $0.002-0.019$ & & & \\
\hline C.V.\% & 14.4 & 27.4 & 58.4 & 31.3 & 14.6 & 20.0 & 111.8 & & & \\
\hline G2 Mean $\pm S D$ & $14.44 \pm 4.18$ & $23.68 \pm 8.93$ & $9.24 \pm 7.56$ & $23.44 \pm 9.13$ & $14.53 \pm 4.01$ & $17.32 \pm 4.69$ & $0.005 \pm 0.003$ & 23 & 3 & Adults \\
\hline Range & $7.18-24.90$ & $11.83-43.68$ & $3.82-28.02$ & $11.83-43.68$ & $7.44-21.22$ & $7.81-24.90$ & $0.002-0.017$ & & & \\
\hline C.V.\% & 27.1 & 37.7 & 81.8 & 38.9 & 27.6 & 27.1 & 76.0 & & & \\
\hline G3 Mean $\pm S D$ & $16.08 \pm 5.03$ & $26.95 \pm 7.82$ & $10.87 \pm 7.72$ & $26.37 \pm 8.22$ & $16.19 \pm 5.11$ & $19.03 \pm 6.6 .30$ & $0.009 \pm 0.015$ & 33 & 4 & 3 Adults \\
\hline Range & $5.30-26.44$ & $12.58-48.10$ & $2.94-34.39$ & $9.77-48.07$ & $5.30-27.34$ & $3.22-27.83$ & $0.002-0.080$ & & & 1 calf** \\
\hline C.V.\% & 31.1 & 29 & 71.0 & 31.2 & 31.6 & 33.1 & 156.9 & & & \\
\hline G4 Mean $\pm S D$ & $14.60 \pm 3.22$ & $24.36 \pm 6.40$ & $9.77 \pm 4.47$ & $24.36 \pm 6.40$ & $14.60 \pm 3.22$ & $18.80 \pm 4.85$ & $0.004 \pm 0.002$ & 8 & 3 & 2 Adults \\
\hline Range & $7.43-17.68$ & $12.07-33.14$ & $4.64-18.14$ & $12.07-33.14$ & $7.43-17.68$ & $9.76-24.41$ & $0.002-0.007$ & & & 1 Juvenile*** \\
\hline C.V.\% & 22.1 & 26.3 & 45.7 & 26.3 & 22.1 & 25.8 & 43.1 & & & \\
\hline G5 Mean $\pm S D$ & $14.53 \pm 3.72$ & $24.39 \pm 9.84$ & $9.84 \pm 7.89$ & $23.80 \pm 10.17$ & $14.66 \pm 3.76$ & $17.94 \pm 6.35$ & $0.008 \pm 0.009$ & 12 & 3 & 2 Adults \\
\hline Range & $7.85-18.31$ & $10.88-41.44$ & $2.99-25.02$ & $10.84-41.44$ & $7.85-18.31$ & $8.79-33.69$ & $0.002-0.030$ & & & 1 Juvenile \\
\hline C.V.\% & 25.6 & 40.4 & 80.2 & 42.7 & 25.7 & 35.4 & 116.2 & & & \\
\hline G6 Mean $\pm S D$ & $8.92 \pm 3.48$ & $16.20 \pm 4.07$ & $7.28 \pm 1.07$ & $16.03 \pm 4.15$ & $8.92 \pm 3.48$ & $11.28 \pm 3.52$ & $0.026 \pm 0.014$ & 9 & 1 & Adult \\
\hline Range & $6.53-17.80$ & $14.13-27.01$ & $6.02-9.21$ & $13.67-27.01$ & $6.53-17.80$ & $7.81-19.53$ & $0.002-0.046$ & & & \\
\hline C.V.\% & 39.0 & 25.1 & 14.8 & 25.9 & 39.0 & 31.2 & 54.4 & & & \\
\hline G7 Mean $\pm S D$ & $13.84 \pm 4.10$ & $27.89 \pm 9.81$ & $14.05 \pm 8.09$ & $19.17 \pm 6.48$ & $22.56 \pm 13.40$ & $18.30 \pm 5.35$ & $0.012 \pm 0.012$ & 7 & 2 & 1Adult \\
\hline Range & $7.06-18.87$ & $13.98-42.99$ & $6.93-28.24$ & $13.98-29.92$ & $7.06-42.99$ & $10.90-21.41$ & $0.002-0.039$ & & & 1 calf \\
\hline C.V.\% & 29.6 & 35.2 & 57.6 & 33.8 & 59.4 & 29.3 & 105.3 & & & \\
\hline G8 Mean $\pm S D$ & $13.83 \pm 2.11$ & $21.83 \pm 2.36$ & $8.0 \pm 2.44$ & $20.51 \pm 2.87$ & $14.93 \pm 2.22$ & $18.42 \pm 2.58$ & $0.010 \pm 0.011$ & 7 & 2 & Adults \\
\hline Range & $11.71-16.66$ & $17.11-24.39$ & $4.01-10.98$ & $16.11-23.44$ & $12.7-18.55$ & $14.65-22.95$ & $0.003-0.033$ & & & \\
\hline C.V.\% & 15.3 & 10.8 & 30.6 & 14 & 14.9 & 14 & 111.9 & & & \\
\hline G9 Mean $\pm S D$ & $13.98 \pm 3.17$ & $26.63 \pm 8.06$ & $9.65 \pm 7.76$ & $22.63 \pm 8.31$ & $15.25 \pm 4.51$ & $17.14 \pm 4.39$ & $0.007 \pm 0.003$ & 13 & 2 & 1 Adult \\
\hline Range & $9.12-19.60$ & $17.11-41.72$ & $3.45-30.35$ & $16.11-41.72$ & $9.12-26.85$ & $11.23-26.86$ & $0.002-0.012$ & & & 1 calf \\
\hline C.V.\% & 22.7 & 34.1 & 80.4 & 36.7 & 29.6 & 25.6 & 45.3 & & & \\
\hline G10 & 17.27 & 34.10 & 17.98 & 27.85 & 23.51 & 23.9320.51- & 0.006 & 2 & 2 & 1Adult \\
\hline Median $\pm S D$ & $15.53-19.01$ & $28.02-40.18$ & $14.78-21.17$ & $15.53-40.18$ & $19.01-28.02$ & 27.34 & $0.004-0.008$ & & & 1 calf \\
\hline $\begin{array}{l}\text { Range } \\
\text { C.V.\% }\end{array}$ & 14.2 & 25.2 & 25.2 & 62.6 & 27.1 & 20.2 & 47.1 & & & \\
\hline G11 Mean $\pm S D$ & 12.51 & 17.66 & 5.15 & 16.11 & 17.66 & 12.51 & 0.008 & 1 & 1 & Adult \\
\hline Range & -- & -- & -- & -- & -- & -- & -- & & & \\
\hline C.V.\% & -- & -- & -- & -- & -- & -- & -- & & & \\
\hline
\end{tabular}


FIG.3

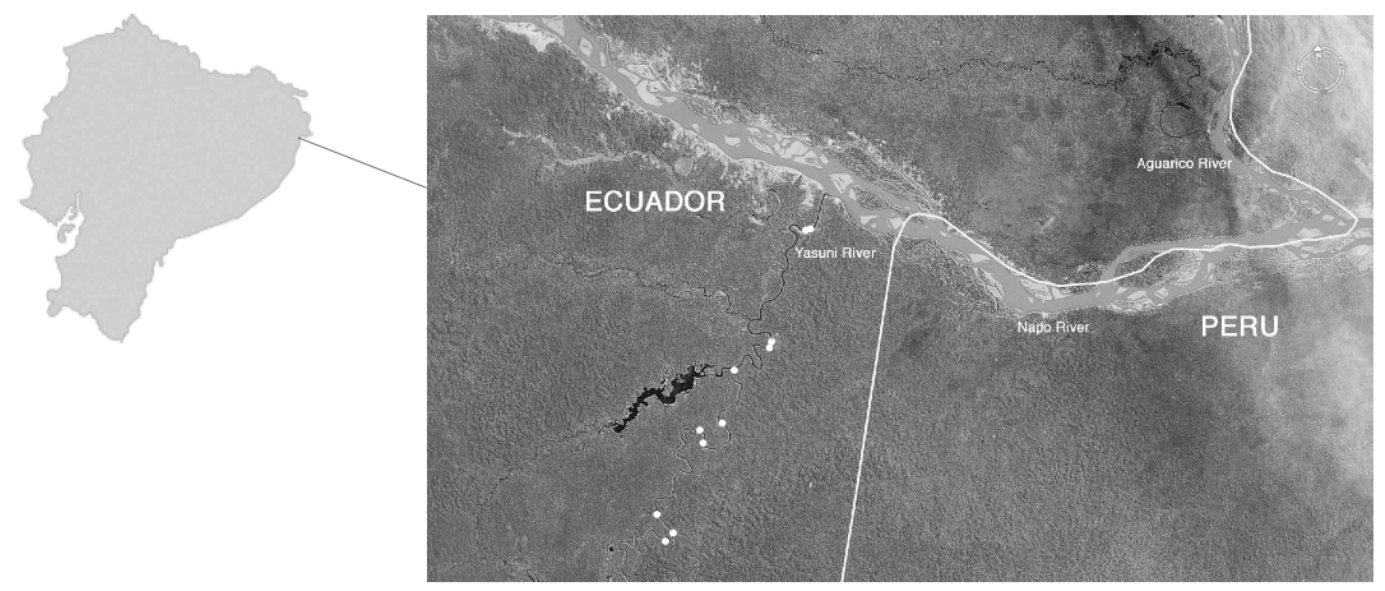


FIG. 4
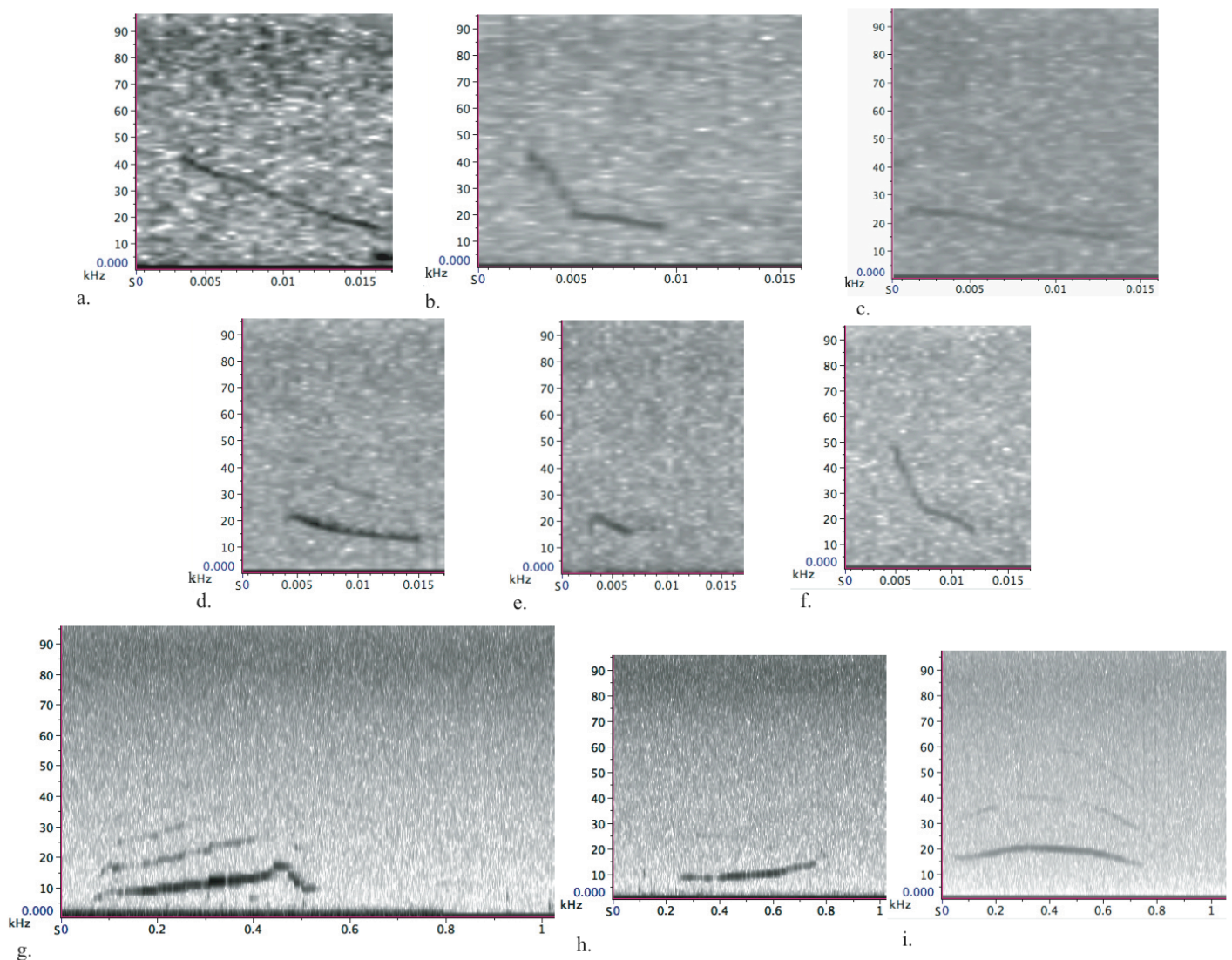
FIG.5
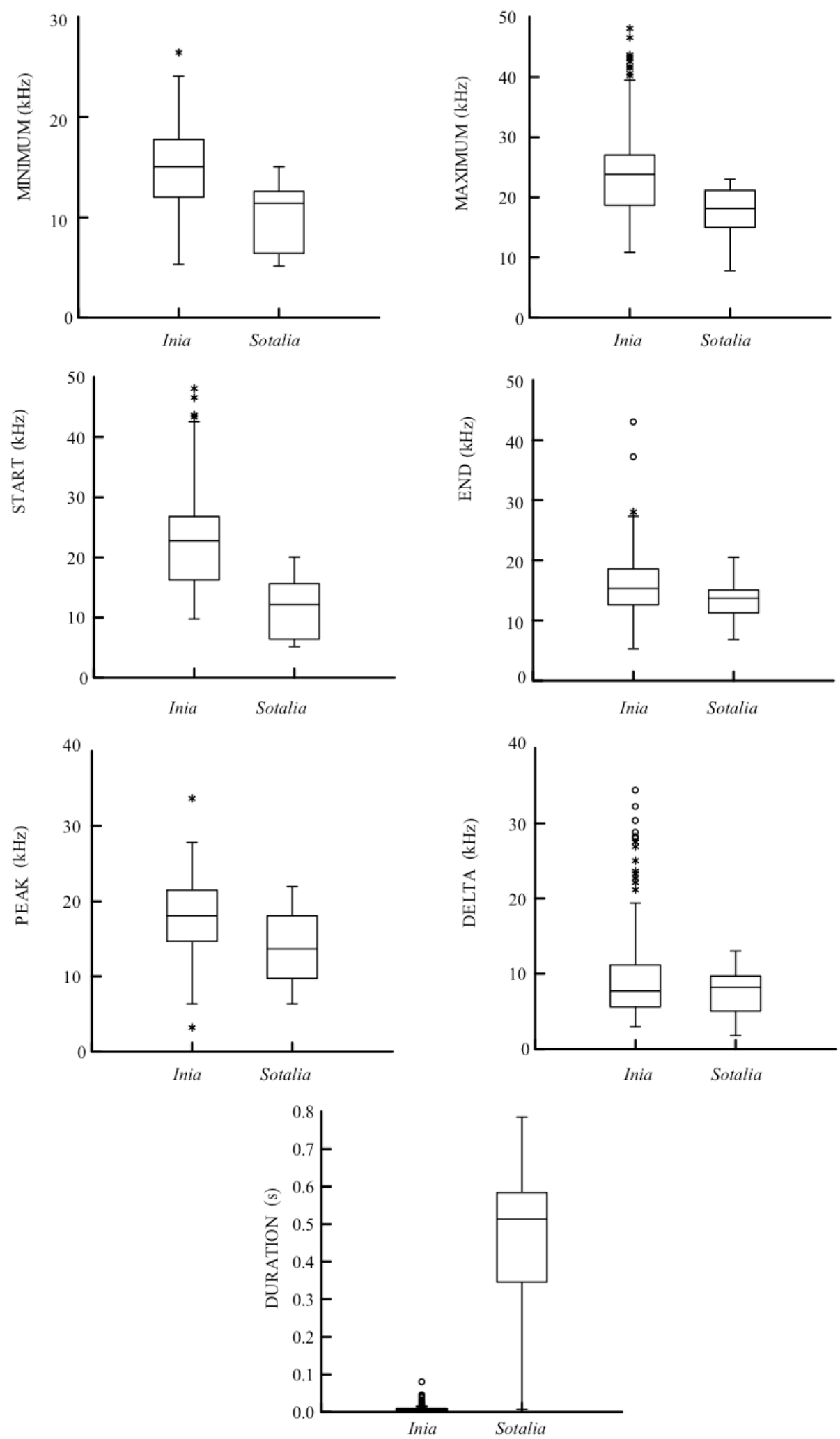


\section{FIG.6}

Inia geoffrensis

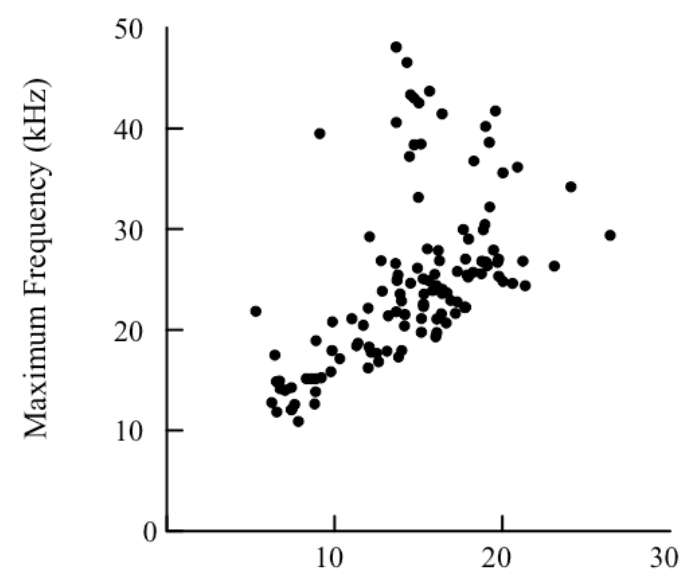

Sotalia fluviatilis

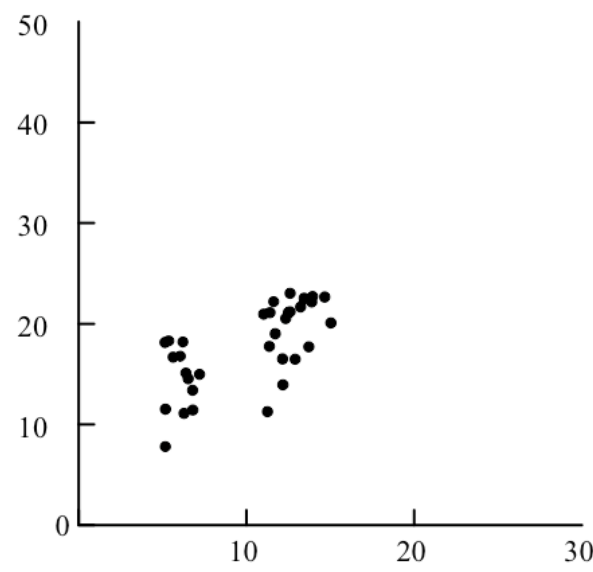

Minimum Frequency $(\mathrm{kHz})$ 


\section{CHAPTER IV}

REEXAMINING THE RELATIONSHIP BETWEEN BODY SIZE AND TONAL

SIGNALS FREQUENCY IN WHALES: A COMPARATIVE APPROACH USING A NOVEL PHYLOGENY

\section{LAURA J. MAY-COLlado ${ }^{1,2}$, Ingi Agnarsson ${ }^{3,4}$, Douglas WARTZOK ${ }^{1}$}

${ }^{1}$ Florida International University, Department of Biological Sciences, $11200 \mathrm{SW} 8^{\text {th }}$ Street, Miami Florida 33199

${ }^{2}$ Universidad de Costa Rica, Escuela de Biología, Apto. 2060 San Pedro, Costa Rica

${ }^{3}$ The University of British Columbia, Departments of Zoology and Botany, 3529-6270 University Blvd., Vancouver, BC., Canada V6T $1 Z 4$.

${ }^{4}$ Systematic Biology-Entomology, E-530, Smithsonian Institution, NHB-105, P.O. Box 37012, Washington, DC 20013-7012, USA

May-Collado, L. J., Agnarsson, I., and D. Wartzok. 2007. Reexamining the relationship between body size and tonal signals frequency in whales: a phylogenetic comparative approach. Marine Mammal Science. Marine Mammal Science. 23 (3): 524-552. 


\begin{abstract}
A negative relationship between cetacean body size and tonal sound minimum and maximum frequencies has been demonstrated in several studies using standard statistical approaches where species are considered independent data points. Such studies, however, fail to account for known dependencies among related species — shared similarity due to common ancestry. Here we test these hypotheses by generating the most complete species level cetacean phylogeny to date, which we then use to reconstruct the evolutionary history of body size and standard tonal sounds parameters (minimum, maximum, and center frequency). Our results show that when phylogenetic relationships are considered the correlation between body size (length or mass) and minimum frequency is corroborated with approximately $27 \%$ of the variation in tonal sound frequency being explained by body size compared to 86 to $93 \%$ explained when phylogenetic relationships are not considered. Central frequency also correlates with body size in toothed whales, while for other tonal sound frequency parameters, including maximum frequency, this hypothesized correlation disappears. Therefore, constraints imposed by body size seem to have played a role in the evolution of minimum frequency while alternative hypotheses are required to explain variation in maximum frequency.
\end{abstract}

Keywords: evolution, adaptation, independent contrast, scaling, communication, phylogeny, tonal signals, toothed-whales, delphinids, Mysticeti. 


\section{INTRODUCTION}

Cetaceans produce an array of sounds that can be broadly categorized as tonal sounds, pulsed sounds, echolocation clicks, and graded sounds (combination of pulsative units and tones) (reviewed by Richardson et al. 1995). Apart from echolocation clicks, tonal signals are among the most studied cetacean sounds. Although similar in their acoustic structure, tonal sounds may be produced by two different mechanisms, possibly laryngeal in baleen whales (Frankel 2002) while in toothed whales sounds are thought to be produced by a complex nasal system (e.g., Cranford et al. 1999, Cranford 2000). In baleen whales tonal signals are narrowband, low in frequency $(<5 \mathrm{kHz})$, and often produced in a stereotypic fashion (Clark 1990, Richardson et al. 1995). These signals are associated with a variety of behavioral contexts such as feeding (in Eubalaena australis, D'Vincent et al. 1985), courtship and group competition on breeding grounds (e.g., in Megaptera novaeangliae, Helweg et al. 1992, Tyack and Whitehead 1983), and other social behaviors (e.g., Eubalaena glacialis, Parks and Tyack 2005). In toothed whales tonal sounds (commonly referred to as 'whistles') have been documented in monodontids (e.g., Karlsen et al. 2002, Shapiro 2006, Sjare \& Smith 1986, Watkins et al. 1970), most delphinids (e.g., Oswald et al. 2003, Rendell et al. 1999, Steiner 1981, Wang et al. 1995a), some ziphiids (e.g., Dawson and Barlow 1998, Manghi et al. 1999, Rogers and Brown 1999), and river dolphin species (Jing et al. 1981, May-Collado and Wartzok 2007, Wang et al. 1995a, 1999, 2001, 2006). Whistles are primarily used in social contexts such as group cohesion, group coordination during feeding, and individual identifiers (e.g., Acevedo-Gutiérrez and Stienessen 2004, Caldwell and Caldwell 1965, Caldwell et al. 1973, Dreher and Evans 1964, Fripp et al. 2005, Herzing 2000, Janik 
2000, Janik et al.1994, Pivari and Rosso 2005, Tyack 1999, 2000, Watwood et al. 2004). Delphinid whistles vary across populations and species: acoustic parameters such as duration and modulation tend to vary intraspecifically (e.g., Barzúa-Durán and Au 2002, 2004, Morisaka et al. 2005a, Wang et al. 1995b) whereas frequency components vary across species (e.g., Matthews et al. 1999, Oswald et al. 2003, Rendell et al. 1999, Steiner 1981, Wang et al. 1995a). Intraspecific variation may result from adaptation to local ecological conditions or geographical isolation and genetic divergence between groups or populations (e.g., Azevedo and Van Sluys 2005, Barzúa-Durán 2004, BarzúaDurán and Au 2004, Morisaka et al. 2005b, Rossi-Santos and Podos 2006, Wang et al. 1995b). In addition there may be variation at a finer scale, such as within-individual, between sexes, groups etc. Interspecific variation in frequency components may additionally be the product of zoogeographic relationships (Steiner 1981), habitat (Wang et al. 1995a), morphological constraints (Matthews et al. 1999, Podos et al. 2002, Wang et al. 1995a,), and phylogenetic relationships (e.g., Steiner 1981, Matthews et al. 1999, Wang et al. 1995a).

Body size is one of the most important morphological factors believed to influence animal signal frequency (Marquet and Taper 1998). Broadly, body size and the size of sound producing organs correlate (Fletcher 1992) and size of vocal tract places physiological constraints on signal production. For example small body-sizes (small sound producing organs) limit animals to the production of relatively high-frequency signals which are more subject to sound attenuation and degradation, limiting the range at which animals can communicate (Gerhardt 1994, Gerhardt and Huber 2002). Some insects and anurans have solved this problem either by using alternative strategies (e.g., 
using plants as acoustic baffles, calling from elevated positions, emitting signals from burrows) or by having structural modifications that allow them to produce lower frequencies (e.g., some grasshoppers, cicadas) (Gerhardt and Huber 2002, Lardner and bin Lakim 2002).

In cetaceans body size has been suggested as a major factor influencing both the maximum and minimum frequency of tonal signals (e.g., Matthews et al. 1999, Podos et al. 2002, Wang et al. 1995a). Using standard statistical methods these studies found a strong negative relationship between body size and maximum frequency (Matthews et al. 1999, Podos et al. 2002, Wang et al. 1995a) and minimum frequency (Matthews et al. 1999 ) with up to $97 \%$ of variation in frequency being explained by body size. However, these methods assume species as independent data points. Felsenstein (1985) emphasized that interspecific comparative studies face the problem of non-independence. Failing to account for known dependencies among related species and recognizing that similarity in size or whistles may be due to common ancestry artificially inflates the number of observations (and degrees of freedom) and correlations or regressions based on such observations are suspect. Correlations imply that a change in the independent trait will result in a change in dependent trait. A single, uniform, large clade of small species with high frequency whistles offers little evidence of correlation as no change is observed in either trait. Of course, these data do not directly contradict the correlation hypothesis, they are just insufficient to strongly test it. That is, when the phylogeny is consulted it becomes clear that the number of valid independent comparisons of values for the two traits is far less than the number of species in the clade. However, if these small species were scattered in the phylogeny among larger lower-pitched species they would provide 
multiple observations of changes in body size accompanied with a change in pitch thus offer stronger support to the hypothesis of correlation. A series of methods have been developed to account for known dependencies among related species using phylogenies (reviewed by Harvey and Pagel 1991, Martins et al. 2002).

The goal of this study is to reevaluate the hypotheses that variation in maximum and/or minimum tonal sound frequency across whales is correlated with body size, and then test more specifically this correlation in toothed-whales with reference to 'whistles', We explore the relationship between several cetacean tonal signal frequency characters and body size using a comparative phylogenetic approach. Our results also cast light on the evolution of body size and the evolutionary history of tonal sounds. 


\section{MATERIALS AND METHODS}

\section{Data and Definitions}

Published data on body size for length (m) and mass (kg), and standard frequency variables of tonal sound $(\mathrm{kHz})$ were obtained from various sources (published literature, personal communications, and L. May-Collado unpublished data) (see Table 1).

Tonal sounds are produced by both baleen whales (Mysticeti) and toothed whales (Odontoceti) and were defined as narrowband sounds that can be relatively constant in frequency (e.g., Lipotes vexillifer, Wang et al. 2006, Sotalia fluviatilis, Azevedo and Van Sluys 2005, Stenella longirostris Barzúa-Dúran and Au 2002, 2004, baleen whales e.g., McDonald et al. 2005, Mellinger and Clark 2003, Watkins et al. 2004) but also greatly

modulated (e.g., Tursiops truncatus, Wang et al. 1995b, Delphinapterus leucas, Karlsen et al. 2002; Lagenorhynchus albirostris, Rasmussen and Miller 2002), show variable duration (e.g., 0.01 to $1.3 \mathrm{sec}$ in Sousa chinensis, Van Parijs and Corkeron 2001), consist of a single or several units, (Richardson et al. 1995) and may or may not contain harmonics (e.g., Lammers and Au 2003, Rasmussen et al. 2006). Throughout we assume authors reported the fundamental frequency and that is what we discuss, since not all state if measurements included harmonics or not. Toothed whale tonal sounds (whistles) have been characterized as generally with fundamental frequencies below $20 \mathrm{kHz}$ (Richardson et al. 1995). However, this upper limit of around $20 \mathrm{kHz}$ in many cases reflects limitations of recording equipment, rather than those of whistle frequency production (e.g., the following studies in dolphins and river dolphins - Wang et al. 1995a,b used a system response up to $24 \mathrm{kHz}$, Corkeron and Van Parijs 2001 up to 22 kHz, Morisaka et al. 2005a,b and Van Parijs et al. 2000 up to $20 \mathrm{kHz}$; in ziphiids - 
Dawson and Barlow 1998 up to $20 \mathrm{kHz}$, and Rogers and Brown 1999 up to $16.5 \mathrm{kHz}$; in belugas - Belikov and Bel'kovich 2001, 2003 up to $20 \mathrm{kHz}$, etc.). Therefore we do not exclude higher frequency whistles such as those produced by some delphinid e.g., Lagenorhynchus albirostris whistles go up to $35 \mathrm{kHz}$ (Rasmussen and Miller 2001) and up to $41 \mathrm{kHz}$ in Tursiops truncatus (Boisseau 2005) or even higher e.g., Inia geoffrensis up to $48 \mathrm{kHz}$ (May-Collado and Wartzok 2007). All tonal sounds considered for baleen whales in this study were those referred to as exclusively tonal. We did not consider sounds that consisted of a combination of pulsative units and tones for either baleen whales (see Heimlich et al. 2005, McDonald et al. 2005, Parks et al. 2005) or toothed whales (see "graded-vocalizations" in Murray et al. 1998).

While focus has traditionally been on toothed whales whistles, we also more broadly examine the optimization of body size and frequency parameters of tonal sounds across cetaceans. It is important to note that the two types of sounds may be produced by different mechanisms (e.g., Cranford et al. 1999, Frankel 2002, Reidenberg and Laitman 2004) and sound production of tonal sounds may well be convergent in baleen whales and toothed whales. However, to rule out their homology, data external to this study would be required. Regardless of homology, body size could similarly constrain frequency in the two types of sounds. Therefore in addition to analyzing them separately, exploring them together as potentially homologous, or as potentially subject to similar constraints, seems worthwhile. 


\section{Phylogenetic analysis and ancestral character reconstruction}

The history of character evolution on the phylogeny (character optimization) was estimated using Mesquite 1.12 (Maddison and Maddison 2006). For this purpose we here produce the most complete species level phylogeny of Cetacea to date by adding two species - the blue whale (Accession number AY235202) and the fin whale (Accession number U13126) - to the phylogeny of May-Collado and Agnarsson (2006). Cytochrome B sequences from Genbank were analyzed in a Bayesian framework using MrBayes 3.1.2 (Ronquist and Huelsenbeck 2003) with model parameters and search strategies as described in May-Collado and Agnarsson (2006). On this phylogeny we optimize body size and whistle frequency using weighted squared-change parsimony (Maddison 1991). Weighted squared-change parsimony minimizes the sum of squared change along all branches of the tree, weighting branches by their length (Maddison and Maddison 2006). Since polytomies (unresolved relationships among lineages) can compromise character optimization and tests of character correlations, characters were optimized on a fully resolved tree, which is the majority rule tree resulting from the MrBayes analysis without collapsing nodes with less than $50 \%$ frequency (using the contype=allcompat option). We mapped the distribution of body length and mass and each of the following standard whistle parameters: maximum, mean maximum, minimum, and mean minimum frequency. We also mapped the distribution of center frequency, although, it is important to note that this parameter is not a direct measurement from the signal itself, but a estimation of central tendency calculated and defined by Matthews et al. (1999) as the mean of $f$ frequency measurements per call. To normalize the data all parameters were natural $\log$ transformed (Sokal and Rohlf 1981). 
Ancestral character reconstruction for each frequency parameter and body size were run separately. This was performed for all species with available tonal frequency parameters and we also ran a separate optimization including all taxa. In species for which we have more than one frequency value in Table 1, we selected the highest for maximum and mean maximum frequency and the lowest for minimum and mean minimum frequency (selected values shown in bold). The maximum reported value for both body length and mass was used for all optimizations. Assuming a normal distribution a mid-point value for all variables optimized in this study would be preferable, but sufficiently detailed data are available only for very few species.

\section{Phylogenetic Comparative Approach: Independent Contrast Method}

To account for dependencies among of species, independent contrasts were calculated for each character. The method makes use of the phylogeny, and a model of evolution (Brownian motion), to estimate the number of independent comparisons between species, or groups of species, that can be used in a regression analysis. For example, a clade of 10 species that are invariable for the characters under study does not constitute 10 independent observations of these characters, instead phylogenetic relationships may explain the character covariation. Independent contrasts were calculated using the PDAP: PDTREE module (Midford et al. 2005) in Mesquite 1.06 (build h47). This module analyzes data using the method of phylogenetically independent contrasts (PIC) developed by Felsenstein (1985). To estimate Felseinstein's independent contrast, branch lengths were used as estimated by MrBayes; branch length transformations were not necessary (Lack of fit test $p>0.05$ for all parameters). The current version of PDAP is 
known to have some error (see Midford et al. 2005) when calculating regressions if some taxa have missing values (unknowns, “?”), although it is unclear how seriously it impacts the analyses. Therefore, in addition to using the full dataset (where some of the taxa lack acoustic data) we also ran analyses on pruned datasets where all species lacking the acoustic character under study were removed prior to the regression analysis. These calculations are known to be correct, however, pruning species from the cladogram affects both estimates of branch lengths and optimization of body size (as available information has been thrown out). While we prefer the pruned analyses, it seems appropriate to report the values based on both types of analyses; the best estimates may lay somewhere in between. 


\section{RESULTS}

In the novel phylogeny (Fig. 1) the newly added blue (Balaenoptera musculus) and fin (B. physalus) whales, as expected are placed within a clade containing other Balaenoptera, as well as Megaptera (humpback whale) and Eschrichtius (grey whale). The fin whale is sister to the humpback whale as also found by Hatch et al. (2006) and Sasaki et al. (2006) while the placement of the blue whale is less well resolved (Fig. 1). As the two are not each other sister taxon, these largest of whales provide independent evidence of change in body size. In other respects this phylogeny is identical, or nearly so, to the phylogeny of May-Collado and Agnarsson (2006).

Of the many changes in body size implied by the phylogeny the most conspicuous are the differences between baleen and toothed whales (Table 1, Fig. 2). Correlated with these changes in body size is change in tonal sound minimum frequency, whether measured as mean minimum or absolute minimum. Body length explains up to $26 \%$ of the variation in minimum frequency across Cetacea and $28 \%$ within toothed whales and up to $66 \%$ of the mean minimum frequency in baleen whales, although this should be interpreted with care as only 4 independent contrasts were regressed (Table 2, Fig.3). When considering body mass, the more commonly used allometric scaling parameter but one more difficult to estimate in cetaceans, minimum frequency (both mean and absolute) across all Cetacea is significantly correlated with biomass $\left(\mathrm{R}^{2}=.135\right.$ for absolute, $\mathrm{R}^{2}=$ .101 for mean). The correlation with mean minimum frequency was also significant within baleen whales, but insignificant within toothed whales, while absolute minimum was not significantly correlated with body mass within each group (marginally insignificant in toothed whales $\mathrm{p}=.052$ ). In contrast, body size explains virtually none 
( $1 \%$ or less) of the variation in maximum frequency across Cetacea and there is no correlation between body size and maximum frequency in any comparisons (Table 2, results are the same using the raw data without log-transformations). The calculated center frequency is significant only within the toothed whales $\left(\mathrm{R}^{2}=.182\right.$ with length, $\mathrm{R}^{2}$ $=.161$ with mass).

The distribution of tonal sounds and optimization of body size across Odontoceti is summarized in Figure 2. The phylogeny broadly implies that cetaceans were primitively large and that there has been a gradual reduction in size in the lineage leading to dolphins and relatives. However, this optimization should be interpreted with care, including fossil data and information from outgroups will be necessary for a detailed account of body size evolution in Cetacea. In addition, this broad pattern addresses only a portion of the variation; there is much variation in body size at the level of families and genera (Fig. 2). Finally there is considerable intraspecific variation in body size, the exploration of which is beyond the scope of this paper. In general the greatest variation in body size is among baleen whales, nevertheless, size variation among the toothed whales is in the range of an order of magnitude in length, and over two orders of magnitude in body mass (Table 1). Size variation in toothed whales significantly correlates with absolute minimum frequency, and central frequency, of their tonal sounds (Table 2). Hence, even if toothed whales whistles are fundamentally different (produced by different mechanisms) from tonal sounds in baleen whales size nevertheless constrains minimum frequency in both sound systems. Independently of body size, high frequency whistles (both in terms of maximum and minimum frequencies) appear to be derived (Fig. 4). 


\section{DISCUSSION}

The new phylogeny is the most detailed phylogenetic hypothesis of whales currently available. It agrees well with most recent studies in cetacean phylogenetics (e.g. Hatch et al. 2006; May-Collado and Agnarsson 2006; Nikaido et al. 2007; Sasaki et al. 2006) and therefore provides an appropriate phylogenetic hypothesis with which to test the correlation of body size and tonal sound frequency in whales.

Even after accounting for phylogenetic relationships the hypothesis that the minimum frequency of whistle, or tonal sounds in general, is negatively correlated with body length (Matthews et al.'s 1999) is corroborated. Body mass is more typically used in these regressions because it is thought to be a more accurate proxy for physiological constraints. However, body mass is more difficult to estimate than body length in cetaceans. When body mass is considered instead of length, only the correlation with absolute minimum frequency is still significant within toothed whales albeit with reduced explanatory power. Our results are congruent with Matthews et al.'s (1999) hypothesis of a significant relationship between central frequency and body length (but not mass) in toothed whales. However, a much smaller percent of frequency variation is explained by body size after accounting for phylogenetic relationships (for minimum frequency about $28 \%$ for toothed whales in our study versus $86-93 \%$ in the study of Matthews et al. 1999).

In contrast, the hypothesis that tonal sound (or whistle) maximum frequency is negatively correlated with body size (Matthews et al. 1999, Podos et al. 2002, Wang et al. 1995a) must be rejected. Even though the phylogeny implies broadly that a major decrease in body size and increase in maximum whistle frequency occurred in the 
common ancestor of pandelphinids (Delphinida sensu Muizon 1998 + Platanista, see May-Collado et al. submitted), that single observation does not imply correlation. In general, throughout the phylogeny, body size and maximum whistle frequency vary independently with only a tiny portion of the variation in maximum frequency being potentially explained by body size (Figs 2-3, Tables 1-2). We should note here that due to limitations of recording systems in some studies (see Methods) the maximum frequency of some species may be underestimated. Hence we cannot rule out that when better information is available results of regression analyses will change. However, we do not expect the effect to be dramatic as we see no correlation of body size and maximum frequency in baleen whales where limitations of equipment is not an issue.

Body size is known to be related to a variety of physiological, ecological, and behavioral processes (Marquet and Taper 1998). In acoustic communication, body size has been acknowledged as a major factor determining signal frequency components. In insects, anurans, birds, and mammals negative relationships between signal frequency and body size (particularly body mass) has been largely supported (e.g., Bennet-Clark 1998, Gerhardt 1994, Hauser 1993, Matthews et al.1999, Palacios and Tubaro 2000, Seddon 2005, Tubaro and Mahler 1998, Wang et al. 1995a, Wiley 1991). However, as more comparative studies consider phylogenetic hypotheses, this relationship in some cases no longer holds (e.g., Farnsworth and Lovette 2005, Laiolo and Rolando 2003). We do find evidence in cetaceans that body size has constrained the evolution of tonal sounds minimum frequency, although size can only explain a portion of the variation. This suggests (1) that in the evolutionary history of whales there has been a selection for low frequency sounds, which e.g. enable communication over long distances, and (2) that the 
degree to which whales have been able to respond to this selection through evolutionary history has been, at least in some cases, constrained by body size. There is no evidence, however, that body size has constrained the evolution of maximum frequency. This certainly does not imply such constraints do not exist, no doubt body size constrains the maximum possible frequencies. What it does imply is that, for maximum frequency, the range of tonal sound frequencies used by cetaceans seems to lie outside the area where physiological constraints would have an impact.

It is right to point out here that, ideally, recordings and body size measurements should come from the same animal, to account for intraspecific size and frequency variation. However, such data are simply not available. Given that body size and minimum frequency correlate even when such detailed evidence are missing, the likely effect of their inclusion would be to increase the amount of variation in minimum frequency explained by body size. We point out that intraspecific variation could, at least in theory, be used as an independent test of these correlations - a study might record and measure multiple individuals within species and explore the intraspecific correlations of body size and frequency. For such a study phylogenetic corrections would not be necessary.

Environmental factors seem to be most important in driving the evolution of acoustic signals in birds, insects, and anurans (e.g., Bertelli and Tubaro 2002, Couldridge and van Staaden 2004, Farnsworth and Lovette 2005, Gerhardt 1994, Laiolo and Rolando 2003, Seddon 2005, Wiley and Richards 1998). This may also be the case in the evolution of cetacean tonal signal frequency as has been suggested by some authors (e.g., Morisaka et al. 2005b, Wang et al. 1995a). Finally, social fluidity is another factor 
suggested to influence tonal frequency within and across species (Bazúa-Durán 2004).

Studies are underway to examine tonal sound evolutionary history taking into considerations some of these factors (May-Collado et al. submitted). 


\section{CONCLUDING REMARKS}

Our results support the negative relationship in cetaceans between body size and minimum tonal sound frequency (whether general tonal sounds, or whistles) as proposed by Matthews et al. (1999). This suggests that there has been a selection for low frequency sounds (enabling e.g. communication over long distances), and that the response to this selection through evolutionary history has been constrained by body size. In contrast, our results do not support the negative relationship between maximum frequency and body size that has been proposed based on a phylogeny-free analysis of the same data (Matthews et al. 1999, and Podos et al. 2002, Wang et al. 1995a). This suggests that if there has been selection for high frequency sounds, body size has not constrained response to it. In this study we focused on tonal signals because these are the best documented sounds in cetaceans. We do not suggest generalizing our findings to other organisms, or even to other cetacean sounds such as echolocation clicks. It is not in dispute that body size imposes absolute constraints on sound production in organisms in general. The question is whether such constraints have come into play in the evolution of sound production in any given lineage. To answer such questions it is invalid to use species as independent data points, and uninformative to allude to constraints observed in other lineages; rather, the lineage of interest should be looked at in isolation using a comparative phylogenetic approach. 


\section{ACKNOWLEDGEMENTS}

We are indebted to Dr. Mike Heithaus and Volker Deecke for their suggestions that improved the manuscript. We thank Peter Midford and Wayne Maddison for their help with the analyses. Thanks to Dr. Maureen A. Donnelly, Dr. Tim Collins, and Dr. Zhenim Chen of Florida International University for their support. Funding for this project came from Judith Parker Travel Grant, Lener- Gray Fund for Marine Research of the American Museum of Natural History, Cetacean International Society, Project Aware, and the

Russell E. Train Education Program-WWF to Laura May-Collado, and NSERC grants to Leticia Aviles and Wayne Maddison, and a Killiam Postdoctoral Fellowship to Ingi Agnarsson'. 


\section{LIST OF REFERENCES}

Acevedo-Guiterrez, A., And S.C. Stienessen. 2004. Bottlenose dolphins (Tursiops truncatus) increase number of whistles when feeding. Aquatic Mammals 30:357-362.

Amano, M. 2002. Finless Porpoise (Neophocoena phocaenoides). Pages 432-35 in W.F. Perrin, B. Wursig and J.G.M. Thewissen, eds. Encyclopedia of Marine Mammals Academic Press, San Diego.

AZEVEDO, A.F., AND SIMÃO, S.M. 2002. Whistles produced by marine tucuxi dolphins

Sotalia fluviatilis in Guanabara Bay, southeastern Brazil. Aquatic Mammals 28:261-266.

AZEVEDO, A.F., AND M. VAN SLuYs. 2005. Whistles of tucuxi dolphins (Sotalia fluviatilis) in Brazil: comparisons among populations. Journal of the Acoustical Society of America 117: 1456-1464.

BAIRD, R. W. 2002. False Killer Whale (Pseudorca crassidens). Pages 411-412 in W.F. Perrin, B. Wursig and J.G.M. Thewissen, eds. Encyclopedia of Marine Mammals Academic Press, San Diego.

BANNISTER, J. L. 2002. Baleen Whales (Mysticetes). Pages 62-72 in W.F. Perrin, B. Wursig and J.G.M. Thewissen, eds. Encyclopedia of Marine Mammals Academic Press, San Diego.

BARZÚA-DURÁN, M. C. 2004. Differences in the whistle characteristics and repertoire of bottlenose and spinner dolphins. Anais da Academia Brasileira de Ciencias 76: 386-392.

BARZÚA-DuRÁN, M. C. 2004. Differences in the whistle characteristics and repertoire of bottlenose and spinner dolphins. Anais da Academia Brasileira de Ciencias 76: 386-392.

BARZÚA-DURÁN, M. C., AND AU, W. W. L. 2002. Whistles of Hawaiian spinner dolphins. Journal of the Acoustical Society of America 112:3064-3072.

BARZÚA-DuRÁN, M. C., AND AU, W. W. L. 2004. Geographic variations in the whistles of spinner dolphins (Stenella longirostris) of the Main Hawaiian Islands. Journal of the Acoustical Society of America 116:3757-3769.

Beasley, I., Robertson, K.M. AND P., ARnOlD. 2005. Description of a new dolphin, the Australian snubfin dolphin Orcaella heinsohni sp. n. (Cetacea, Delphinidae). Marine Mammal Science 21: 365-400.

BELIKov, R. A., AND V. M. BEL'KOVICH. 2001. Characteristics of white sea beluga whale (Delphinapterus leucas Pall) whistle-like signals. XI Session of the Russian Acoustical Society, Moscow, November 19-23. 
BELIKOV, R. A., AND V. M. BEL'KOVICH. 2003. Underwater vocalization of the beluga whales (Delphinapterus leucas) in a reproductive gathering in various behavioural situations. Oecologia. 43:112-120.

BenNet-Clark, H. C. 1998. Size and scale effects as constraints in insect sound communication. Philosophical Transactions: Biological Sciences. 353:407-419.

Bertelli, S., AND Tubaro, P. L. 2002. Body mass and habitat correlates of song structure in a primitive group of birds. Biological Journal of the Linnean Society 77:423430.

Bjørge, A., And K. A.Tolley. 2002. Harbor Porpoise (Phocoena phocoena). Pages 549-551 in W.-F. Perrin, B. Wursig and J.G.M. Thewissen, eds. Encyclopedia of Marine Mammals Academic Press, San Diego.

BoISSEAU, O. 2005. Quantifying the acoustic repertoire of a population: the vocalizations of free-ranging bottlenose dolphins in Fiordland, New Zealand. Journal of the Acoustical Society of America 117:2318-2329.

BusnelL, R.-G., AND A. DzIEDZIC. 1968. Caracteristiques physiques de certains signaux acoustiques du delphidide Steno bredanensis, Lesson. Comptes reduns del'Académie des Sciences Paris Series D. 262:143-146.

Caldwell, M.C., AND CAlDwell, D.K. 1965. Individual whistle contours in bottlenose dolphins (Tursiops truncatus). Nature (Lond.), 207: 434-435.

Caldwell, M. C., Caldwell, D. K. And J. F. Miller. 1973. Statistical evidence for individual signature whistles in the spotted dolphin, Stenella plagiodon. Cetology 16:121.

CiPriano, F. 2002. Atlantic White-Sided Dolphin (Lagenorhynchus acutus). Pages 49-50 in W.F. Perrin, B. Wursig and J.G.M. Thewissen, eds. Encyclopedia of Marine Mammals Academic Press, San Diego.

CLARK. C.W. 1990. Acoustic behavior of mysticete whales. Pages 571-583 in J. Thomas and R. A. Kastelein, eds. Sensory abilities of cetaceans. Plenum Press, New York,

Clark, C.W., AND J. H. Johnson. 1984. The sounds of the bowhead whale, Balaena mysticetus, during the spring migrations of 1979 and 1980. Canadian Journal of Zoology 62:1436-1441.

Corkeron, P.J., AND VAn PARIJs, S.M. 2001. Vocalizations of eastern Australian Risso's dolphins, Grampus griseus. Canadian Journal of Zoology 79: 160-164. 
COUlDRIDGE, V.C.K., AND M.J. VAN STAADEN. 2004. Habitat-dependent transmission of male advertisement calls in bladder grasshopper (Orthoptera; Pneumoridae). Journal of Experimental Biology 207: 2777-2786.

CRANFORD, W. 2000. In search of impulse sound sources in Odontocetes. Pages 156-224 in W.W.L. Au, A.N. Popper, and R.R. Fay, eds. Hearing by Whales and Dolphins, Springer Press, London.

CRANFOrd W., AmUndin M., AND NORRIS K. S. 1999. Functional morphology and homology in the odontocete nasal complex: implications for sound generation. Journal of Morphology 228, 223-285.

CRespo, E.A. 2002. Franciscana (Pontoporia blainvillei). Pages 482-484 in W.F. Perrin, B. Wursig and J.G.M. Thewissen, eds. Encyclopedia of Marine Mammals Academic Press, San Diego.

Cummings, W.C., Thompson, P.O., And R. CoOK. 1968. Underwater sounds of migrating gray whales, Eschrichtius glaucus. Journal of the Acoustical Society of America 44:1278-1281.

Cummings, W.C., Thompson, P.O., And S. J. HA. 1986. Sounds from Bryde's, Balaenoptera edeni, finback, B. physalus, whales in the Gulf of Californai. Fish Bulletin U. S. 84:359-370.

Cunha H.A., Da Silva, V.M.F, Lailson-Brito JR, J., Santos M.C.O., Flores, P.A.C., Martin, A.R., Azevedo A.F., Fragoso, A.B.L., Zanelatto, R.C. And A.M. SOLE'-CAVA. 2005. Riverine and marine ecotypes of Sotalia dolphins are different species. Marine Biology. 148: 449-457

DAHLHEIM, M.E., AND AWBREY, F. 1982. A classification and comparison of vocalizations of captive killer whales (Orcinus orca). Journal of the Acoustical Society of America 72:661-670.

DAHLheim, M.E., FiSHER, H.D., AND SCHEMPP J. D. 1984. Sound production by the gray whale and ambient noise levels in Laguna San Ignacion, Baja California Sur, Mexico. In M.L., Jones, L. Swartz and S. Leatherwood, eds. The Gray Whale. Academic Press, New York.

DA SILVA, V.M.F. 2002. Amazon River Dolphin (Inia geoffrensis). Pages 18-20. in W.F. Perrin, B. Wursig and J.G.M. Thewissen, eds. Encyclopedia of Marine Mammals Academic Press, San Diego.

DAwBIN, W.H., AND D.H. CATO. 1992. Sounds of a pygmy right whale (Caperea marginata). Marine Mammal Science 8:213:219. 
DAwson, S.M. 2002.Cephalorhynchus Dolphins (Cephalorhynchus spp.). Pages 200-203 in W.F. Perrin, B. Wursig and J.G.M. Thewissen, eds. Encyclopedia of Marine Mammals Academic Press, San Diego.

DAWSON, S., AND J. BARLOW. 1998. Sounds recorded from Baird's beaked whale, Berardius bairdii. Marine Mammal Science 14:335-344.

Diazgranados, M.C., AND F. TRUJILlO. 2002. Vocal repertoire of the freshwater dolphins Inia geoffrensis and Sotalia fluviatilis in Colombia, South America. Journal of the Acoustical Society of America 112: 2400.

Dolar, M.L.L. 2002. Fraser's Dolphin (Lagenodelphis hosei). Pages 485-486 in W.F. Perrin, B. Wursig and J.G.M. Thewissen, eds. Encyclopedia of Marine Mammals Academic Press, San Diego.

DreHER, J.J., AND W.E. Evans. 1964. Cetacean communication. Pages 373-399 in W.N. Tavolga, ed. Marine Bioacoustics. Pergamon Press, Oxford.

Driscoll, A.D. 1995. The whistles of Hawaiian spinner dolphins, Stenella longirostris, Master's thesis, University of California at Santa Cruz.

D’VinCENT, C.G., Nilson, R.M., AND HANNA, R.E. 1985. Vocalization and coordinated feeding behavior of the humpback whale in southeastern Alaska. The Scientific Reports of the Whales Research Institute Vol. 36.

EDDS, P. 1988. Characteristics of finback, Balaenoptera physalus, vocalizations in the St. Lawrence Estuary. Bioacoustics. 1:131-149.

EDDS, P. 1993 Vocalisations of a captive juvenile and free-raning adult-calf pairs of Bryde’s whales, Balaenoptera edeni. Marine Mammal Science 9: 269-284.

ERBER, C., AND SimÃo, S. M. 2004. Analysis of whistles produced by the tucuxi dolphin Sotalia fluviatilis from Sepetiba Bay, Brazil. Annals of the Brazilian Academy of Sciences 76:381-385.

FARNSWORTH, A., AND I.J. LOVETTE. 2005. Evolution of nocturnal flight calls in migrating wood-warblers: apparent lack of morphological constraints. Journal of Avian Biology 36:337-347.

Felsenstein, J. 1985. Phylogenies and the comparative method. American Naturalist 125: $1-15$.

FiSH, J.F., SuMICH, J.L., AND G.L. LinGLE. 1974. Sounds produced by the gray whale, Eschrichtius robustus. Marine Fisheries Review 36:38-45. 
Fletcher, N. H. 1992. Acoustic Systems in Biology. Oxford University Press, Oxford, England. $352 \mathrm{pp}$.

Flores, P.A.C. 2002. Tucuxi Sotalia fluviatilis. Pages 1267-1269 in W.-. Perrin, B. Wursig and J.G.M. Thewissen, eds. Encyclopedia of Marine Mammals Academic Press, San Diego.

FORD, J.K.B. 1989. Acoustic behavior of resident killer whales (Orcinus orca) off Vancouver Island, Bristish Columbia (Canada). Canadia Journal of Zoology 67:727-745.

FORD, J.K.B., AND H.D. FISHER. 1978. Underwater acoustic signals of the narwhal (Monodon monocerus). Canadian Journal of Zoology 56:552-560.

Frankel, A. S. 2002. Sound Production. Pages 1126-1137 in W.F. Perrin, B. Wursig and J.G.M. Thewissen, eds. Encyclopedia of Marine Mammals Academic Press, San Diego.

Fripp D., Owen C., Quintana-Rizzo E., Shapiro A., Buckstaff K., Jankowski K., WELLS R., AND P. TYACK. 2005. Bottlenose dolphin (Tursiops truncatus) calves appear to model their signature whistles on the signature whistles of community members. Animal Cognition 8:17-26.

GERHARDT, H. C. 1994. The evolution of vocalization in frogs and toads. Annual Review of Ecology and Systematics 25:293-324.

GERHARDT, H.C., AND F. HUBER 2002.Communication in Insects and Anurans: common problems and diverse solutions. The University of Chicago Press, Chicago. 531 pp.

Goodall, R. N. 2002a. Spectacled Porpoise (Phocoena dioptrica). Pages 1158-1161 in W.F. Perrin, B. Wursig and J.G.M. Thewissen, eds. Encyclopedia of Marine Mammals Academic Press, San Diego.

Goodall, R. N. 2002b. Peale's Dolphin (Lagenorhynchus australis). Pages 890-894 in W.F. Perrin, B. Wursig and J.G.M. Thewissen, eds. Encyclopedia of Marine Mammals Academic Press, San Diego.

GoOdAlL, R. N. 2002c. Hourglass Dolphin (Lagenorhynchus cruciger). Pages 583-585 in W.F. Perrin, B. Wursig and J.G.M. Thewissen, eds. Encyclopedia of Marine Mammals Academic Press, San Diego.

Hafner, G.W., Hamilton, C.L., Steiner, W.W., Thompson, T.J., AND H.E. WinN. 1979. Signature information in the song of the humpback whale. Journal of the Acoustical Society of America 66:1-6. 
Harvey, P. H., AND M. D. PAgel. 1991. The comparative method in evolutionary biology. Oxford Univ. Press, Oxford, U.K. 248 pp.

Hatch L.T., Dopman, E.B., AND R.G. Harrison. 2006. Phylogenetic relationships among the baleen whales based on maternally and paternally inherited characters. Molecular Phylogenetics and Evolution 41:12-27.

HAUSER, M. D. 1993. The evolution of nonhuman primate vocalizations: effects of phylogeny, body weight and social context. American Naturalists 142:528-542.

HeIDE-Jørgensen, M. P. 2002. Narwhal (Monodon monocerus). Pages 783-787 in W.F. Perrin, B. Wursig and J.G.M. Thewissen, eds. Encyclopedia of Marine Mammals Academic Press, San Diego.

Heimlich, S.L., Mellinger, D.K., Nieukirk, S.L., AND C.G. Fox. 2005. Types, distribution, and seasonal occurrence of sounds attributed to Bryde's whales (Balaenoptera edeni) recorded in the eastern tropical Pacific, 1999-2001. Journal of the Acoustical Society of America 118:1830-1837.

Helweg, D.A., Frankel, A.S., Mobley, J.R., and Herman, L.M. 1992. Humpback whale song: our current understanding. Pages 459-483 in J.A. Thomas, R.A. Kastelein, and A.Y. Supin, eds. Marine Mammal Sensory Systems. Plenum, New York.

HERZING, D. L. 2000. Acoustics and social behavior of wild dolphins: implications for a sound society. Pages 225-272 in W.W.-L., Au Popper, A.N. and R.E. Fay, eds. Hearing by whales and dolphins. Springer Press, London.

JANIK, V. M. 2000. Whistle matching in wild bottlenose dolphins (Tursiops truncatus). Science 289:1355-1357.

JANIK, V.M., DEHNHARDT, G., AND TODT, D. 1994. Signature whistle variations in bottlenosed dolphin, Tursiops truncatus. Behavioral Ecology and Sociobiology 35:243248.

Jefferson, T. A. 2002a. Dall's Porpoise (Phocoenoides dalli). Pages 308-310 in W.F. Perrin, B. Wursig and J.G.M. Thewissen, eds. Encyclopedia of Marine Mammals Academic Press, San Diego.

JefFerson, T. A. 2002b. Clymene Dolphin (Stenella clymene). Pages 234-236 in W.F. Perrin, B. Wursig and J.G.M. Thewissen, eds. Encyclopedia of Marine Mammals Academic Press, San Diego.

JEFFERSON, T. A. 2002c. Rough-Toothed Dolphin (Steno bredanensis). Pages1055-1059 in W.F. Perrin, B. Wursig and J.G.M. Thewissen, eds. Encyclopedia of Marine Mammals Academic Press, San Diego. 
JefFerson, T. A. AND B. E. Curry. 2003. Stenella clymene. Mammalian Species 726:15.

JinG, X., Youfo, X. AND J. RongCAI. 1981. Acoustic signals and acoustic behavior of the Chinese River Dolphin (Lipotes vexillifer). Scientia Sinica 24:407-415.

Jones, M. L. AND S. L. Swartz. 2002. Gray Whale. Pages 524-537 in W.F. Perrin, B. Wursig and J.G.M. Thewissen, eds. Encyclopedia of Marine Mammals Academic Press, San Diego.

KaIYA, Z. 2002. Baiji (Lipotes vexillifer). Pages 58-61in W.-F. Perrin, B. Wursig and J.G.M. Thewissen, eds. Encyclopedia of Marine Mammals Academic Press, San Diego.

Karlsen, J.D., Bisther A, Lydersen, C., Haug, T., AND K.M. Kovacs. 2002. Summer vocalisations of adult male white whales (Delphinapterus leucas) in Svalbard, Norway. Polar Biology 25:808-817.

Knowlton, A.R., Clark, C.W., And S.D. Kraus. 1991. Sounds recorded in the presence of sei whales (B. borealis). Abstract $9^{\text {th }}$ Biennial Conference on the Biology of Marine Mammals, p.40. Chicago.

LAMMERs, M. O., AND AU, W. W. L. 2003. Directionality in the whistles of Hawaiian spinner dolphins (Stenella longirostris): a signal feature to cue direction of movement?" Marine Mammal Science 19: 249-264.

LARDNER, B. AND M. BIN LAKIM, 2002. Tree-hole frogs exploit resonance effects. Nature (Lond.) 420: 475.

LAiolo, P. AND Rolando, A. 2003. Comparative analysis of the rattle calls in Corvus and Nucrifraga: the effect of body size, bill size, and phylogeny. Condor 105:139-144.

Leatherwood, S., Jefferson, T.A., Norris, J.C., Stevens, W.E., Hansen, L.J., AND K.D. MuLLIN. 1993. Occurrence and sounds of Fraser's dolphins (Lagenodelphis hosei) in the gulf of Mexico. The Texas Journal of Science. 45:349-354.

LIPSKY, J. D. 2002. RIGHT Whale dolphins (Lissodelphis borealis and Lissodelphis peronii). Pages1030-1032 in W.F. Perrin, B. Wursig and J.G.M. Thewissen, eds. Encyclopedia of Marine Mammals Academic Press, San Diego.

LJUngBlad, D.K., ThOMPson, P.O., AND MoORE S.E. 1982. Underwater sounds recorded from migrating bowhead whales, Baleana mysticetus, in 1979. Journal of the Acoustical Society of America 71:477. 
LJungblad, D.K., Stafford. K. M, And H. ShimadA. 1997. Sound attributed to blue whales recorded off the southwest coast of Australia in December 1995. Reports of the International Whaling Commission 47: 435-439.

MADDISON, W.P. 1991. Square-change parsimony reconstructions of ancestral states for continuous-valued characters on a phylogenetic tree. Systematic Zoology 40: 304-314.

MAdDISON, W.P., AND D.R. MAdDISON. 2006. Mesquite: A modular system for evolutionary analysis. Version 1.12. http://mesquiteproject.org

Manghi, M., Montesi, G., Fossati, C., Pavan, G., Priano, M., And Teloni, V. 1999. Cuvier's beaked whales in the Ionian Sea: First recordings of their sounds. European Research on Cetaceans 13:39-42.

MARQUeT, P.O., AND M.L. TAPER. 1998. On size and area: patterns of mammalian body size extreme across landmasses. Evolutionary Biology 12:127-139.

Martins, E.P., Diniz, J.A.F., AND E.A. Housworth. 2002. Adaptive constraints and the phylogenetic comparative method: a computer simulation test. Evolution 56:1-13.

Matthews, J.N., Rendell, L.E., Gordon, J.C.D., And MacDonald, D.W. 1999. A review of frequency and time parameters of cetacean tonal calls. Bioacoustics 10: 47-71. 21

May-Collado, L.J. ANd I. Agnarsson. 2006. Cytochrome b and Bayesian inference of whale phylogeny. Molecular Phylogenetics and Evolution. 38: 344-354

MAY-COLlADO, L.J., AND D. WARTZOK. 2007.The freshwater dolphin Inia geoffrensis geoffrensis produces high frequency whistles. Journal of Acoustical Society of America. 121 (2): 1203-1212.

May-Collado, L.J., Agnarsson, I., And D. WartzoK. Phylogenetic review of tonal sound production in whales in relation to sociality. Submitted to BMC Evolutionary Biology.

McDonald, M.A., Hildebrand J.A., Wiggins, S.M., Thiele, D., Glasgow, D., And S.E. MoORE. 2005. Sei whale sounds recorded in the Antarctic. Journal of the Acoustical Society of America 118:3941-3945.

MEAD, J. G. 2002. Shepherd's beaked whale (Tasmacetus shepherdi). Pages 1078-1080 in W.F. Perrin, B. Wursig and J.G.M. Thewissen, eds. Encyclopedia of Marine Mammals Academic Press, San Diego.

Mellinger, D. K. AND C. W. Clark. 2003. Blue whale (Balaenoptera musculus) sounds from the North Atlanctic. Journal of the Acoustic Society of America. 114:1108-1119. 
Midford, P.E., T. GARLAND JR., AND W.P. MAdDISON. 2005. PDAP Package of Mesquite.

MiYASAKi, N. AND W. F. PERrin.1994. Rough-toothed dolphin- Steno bredanensis (Lesson 1828). Pages 1-22, Volume 5 in S.H. Ridgway and Sir H. Harrison, eds. Hanbook of Marine Mammals. Academic Press, London.

MoORE, S.E., AND S.H. RIDGWAY. 1995. Whistles produced by common dolphins from Southern California Bight. Aquatic Mammals 21:55-63.

Morisaka, T., Shinohara, M., NAKahara, F. And T. AKamatsu. 2005a. Effects of ambient noise in the whistles of Indo-Pacific bottlenose dolphin Tursiops aduncus populations in Japan. Journal of Mammalogy 86:541-546.

Morisaka, T., Shinohara, M., Nakahara, F., And T. Akamatsu. 2005b. Geographic variations in the whistles among three Indo-Pacific bottlenose dolphin Tursiops aduncus populations in Japan. Fisheries Science 71:568-576.

Muizon, C. DE. 1988. Les relations phylogénétiques des Delphinida. Annales de Paleontologie 74:157-227.

Mullin K.D., Higgins, L.V., JefFerson, T.A., And L.J. Hansen. 1994. Sightings of the Clymene dolphin (Stenella clymene) in the Gulf of Mexico. Marine Mammal Science $10: 464-470$.

Murray, S.O., Mercado, E., AND H.L. Roitblat. 1998. Characterizing the structure of false killer whale (Pseudorca crassidens) vocalizations. Journal of the Acoustical Society of America 104:1679-1688.

NiKAidO, M., PisKuREK, O. AND N. OKADA. 2007. Toothed whale monophyly reassessed by SINE insertion analysis: the absence of lineage sorting effects suggests a small population of a common ancestral species. Molecular Phylogenetics and Evolution. 23:216-224.

O’Corry-Crowe, G. M. 2002. Beluga Whale (Delphinapterus leucas). Pages 94-99 in W.F. Perrin, B. Wursig and J.G.M. Thewissen, eds. Encyclopedia of Marine Mammals Academic Press, San Diego.

OSWALD J.N., BARLOW J., AND T.F. NORRIS. 2003.Acoustic identification of nine delphinids species in the eastern tropical Pacific Ocean. Marine Mammal Science 19:2037.

Oswald, J.N., Rankin, S., AND J. Barlow. Submitted. First description of whistles of Pacific Fraser's dolphins, Lagenodelphis hosei. Bioacoustics. 
Palacios, M.G., AND Tubaro, P.L. 2000. Does beak size affect acoustic frequencies in woodcreepers? Condor 102: 553-560.

PARKS, S.E., AND P.L. TYACK. 2005. Sound production by North Atlantic right whales (Eubalaena glacialis) in surface active groups. Journal of the Acoustical Society of America. 117: 3297-3306.

PERrin, W.F. 2002a. Common Dolphins (Delphinus delphis). Pages 245-248 W.F. Perrin, B. Wursig and J.G.M. Thewissen, eds. Encyclopedia of Marine Mammals Academic Press, San Diego.

PERRIN, W.F. 2002b. Pantropical spotted dolphins (Stenella attenuata). Pages 865-867 W.F. Perrin, B. Wursig and J.G.M. Thewissen, eds. Encyclopedia of Marine Mammals Academic Press, San Diego.

PERrIN, W.F. 2002c. Atlantic spotted dolphin (Stenella frontalis). Pages 47-49 W.F. Perrin, B. Wursig and J.G.M. Thewissen, eds. Encyclopedia of Marine Mammals Academic Press, San Diego.

PERRIN, W.F. 2002d. Spinner dolphin (Stenella longirostris). Pages 1174-1178 W.F. Perrin, B. Wursig and J.G.M. Thewissen, eds. Encyclopedia of Marine Mammals Academic Press, San Diego.

Perrin, W.F., Wursig, B., AND J.G.M. Thewissen. 2002. Encyclopedia of Marine Mammals. Academic Press, San Diego. 1414 pp.

Perryman, W.L. 2002. Melon-headed whale. Pages 733-734 W.F. Perrin, B. Wursig and J.G.M. Thewissen, eds. Encyclopedia of Marine Mammals Academic Press, San Diego.

Pitman, R.L. 2002a. Mesoplodont Whales (Mesoplodon spp). Pages 738-742 W.F. Perrin, B. Wursig and J.G.M. Thewissen, eds. Encyclopedia of Marine Mammals Academic Press, San Diego.

Pitman, R.L. 2002b. Indo-Pacific beaked whale (Indopacetus pacificus). Pages 615-624 W.F. Perrin, B. Wursig and J.G.M. Thewissen, eds. Encyclopedia of Marine Mammals Academic Press, San Diego.

PIVARI, D. AND S. Rosso. 2005. Whistles of small groups of Sotalia fluviatilis during foraging behavior in Southeastern Brazil. Journal of the Acoustical Society of America. 118: 2725-2731.

Podos, J., DA SilVA, V.M.F., AND M.R. Rossi-SANTOS. 2002. Vocalizations of Amazon river dolphins, Inia geoffrensis: insights into the Evolutionary origins of delphinid whistles. Ethology 108, 601-612. 
Rasmussen, M. H., AND Miller, L. A. 2002. Whistles and clicks from white-beaked dolphins, (Lagenorhynchus albirostris Gray 1846) recorded in Faxafloi Bay, Iceland.Aquatic Mammals 28: 78-89.

Rasmussen, M. H., Lammers, M., Beedholm, K., And Miller, L. A. 2006. Source levels and harmonic content of whistles in white-beaked dolphins (Lagenorhynchus albirostris)". Journal of the Acoustical Socitey of America 120: 511-517.

Reidenberg, J. S., And J. T. Laitman. 2002. Prenatal development in Cetaceans. Pages 998-1007 W.-F. Perrin, B. Wursig and J.G.M. Thewissen, eds. Encyclopedia of Marine Mammals Academic Press, San Diego.

REIDENBERG, J. S., AND J. T. LAITMAN. 2004. Anatomy of infrasonic communication in baleen whales: divergent mechanisms of sound generation in mysticetes and odontocetes. The Journal of the Acoustical Society of America 115:2556.

Rendell, L.E., Matthews, J.N., Gill, A., Gordon, J.C.D., And MacDonald, D.W. 1999. Quantitative analysis of tonal calls from five odontocete species, examining interspecific and intraspecific variation. Journal of Zoology 249: 403-410.

REYES, J. C. 2002. Burmeister's porpoise (Phocoena spinipinnis). Pages 549-552 in W.F. Perrin, B. Wursig and J.G.M. Thewissen, eds. Encyclopedia of Marine Mammals Academic Press, San Diego.

Richardson, W.J., Greene, C.R.J., Malme, C.I., And Thomsom, D.H.1995. Marine Mammals and Noise. Academic Press, New York. 576 pp.

RIESCH, R., FORD, J.K.B., AND F. THOMSEN. 2006. Stability and group specificity of stereotyped whistles in resident killer whales, Orcinus orca, off British Columbia. Animal Behavior 71:79-91.

Rogers, T. L. AND S. M., BROWN.1999. Acoustic observations of Arnoux's beaked whale (Berardius arnuxii) off Kemp Land, Antarctica. Marine Mammal Science 15:192198

Rojas-Brucho, L., AND A. JARAMILlO-LegorRetA. 2002. Vaquita (Phocoena sinus). Pages 1277-1279 in W.F. Perrin, B. Wursig and J.G.M. Thewissen, eds. Encyclopedia of Marine Mammals Academic Press, San Diego.

Ronquist, F., AND HuelsenBeck J. P. 2003. MRBAYES 3: Bayesian phylogenetic inference under mixed models. Bioinformatics 19:1572-1574.

Ross, G.J.B. 2002. Humpback dolphins (Sousa chinensis, S. plumbea, and S. teuszi). Pages 585-589 in W.F. Perrin, B. Wursig and J.G.M. Thewissen, eds. Encyclopedia of Marine Mammals Academic Press, San Diego. 
Rossi-SAnTOS, M. R. AND J. Podos. 2006. Latitudinal variation in whistle structure of the estuarine dolphin Sotalia guianensis. Behaviour 143:347-364.

Rugh, D.J., AND K.E.W. SHELdEN. 2002. Bowhead whale (Balaena mysticetus). Pages 129-130 in W.F. Perrin, B. Wursig and J.G.M. Thewissen, eds. Encyclopedia of Marine Mammals Academic Press, San Diego.

Sasaki, T., Nikaido, M., Wada, S. Yamada T.K, CaO, Y., Hasegawa, M. And N. OKADA. 2006. Balaenoptera omurai is a newly discovered baleen whale that represents an ancient evolutionary lineage. Molecular Phylogenetics and Evolution 41:40-52

SchulTZ, K.W., AND P.J. CORKERON. 1994. Interspecific differences in whistles produced by inshore dolphins in Moreton Bay, Queensland, Australia. Canadian Journal of Zoology 72: 1061-1068

SEDDON, N. 2005. Ecological adaptation and species recognition drives vocal evolution in neotropical suboscine birds. Evolution 59:200-215.

SHAPIRO, A. 2006.Preliminary evidence for signature vocalizations among free-ranging narwhals (Monodon monocerus). Journal of the Acoustical Society of America 120: 1695-1705.

Sirovic, A., Hildebrand, J. A., Wiggins, S. M., McDonald, M. A., Moore, S. E., AND D. THIELE. 2004. Seasonality of blue and fin whales calls and the influence of sea ice in the Western Antarctic Peninsula. Deep-Sea Research II. 51:2327-2344.

SJARE, B.L., AND T.G. SMITH. 1986. The vocal repertoire of white whales, Delphinapterus leucas, summering in Cunningham Inlet, Northwest Territories. Canadian Journal of Zoology 64:407-415.

SOKAL, R. R., AND F. J. ROHLF.1981. Biometry: The principles and practice of statistics in Biological Research. $2^{\text {nd }}$ Edition. W.H. Freeman and Company, San Francisco. 859 pp.

StAFFORD, K. M. 1994. Acoustic detection and location of blue whales (Balaenoptera musculus) from SOSUS by matched filtering. Journal of the Acoustical Society of America. 96:3250.

StAFFord, K. M., NiCUKIRK, S. L., AND C. G. FoX. 2001. Geographic and seasonal variation of blue whale calls in the North Pacific. Journal of Cetacean Research and Management. 3:65-76.

STEINER, W. W. 1981. Species-specific differences in pure tonal whistle vocalizations of five western North Atlantic dolphin species. Behavioral Ecology Sociobiology 9:241246. 
Steiner, W.W., Hain, J.H., Winn, H.E., AND P. J. Perkins. 1979. Vocalizations and feeding behavior of the killer whale (Orcinus orca). Journal of Mammalogy 60:823-827.

Thomsen, F., Franck D., AND J.K.B. Ford. 2001. Characteristics of whistles from the acoustic repertoire of resident killer whales (Orcinus orca) off Vancouver Island, British Columbia. Journal of the Acoustical Society of America 109:1240-1246.

ThOMPSON, P. O., AND FRIEDL, W. A. 1982. A long term study of low frequency sounds from several species of whales off Oahu, Hawaii. Cetology. 45: 1-19.

Tubaro, P.L., AND B. Mahler. 1998. Acoustic frequencies and body mass in new world doves. Condor 100:54-61.

TYACK, P.L. 1999: Communication and cognition. Pages 304-306 in J.E. Reynolds and S. A. Rommel, eds. Biology of Marine Mammals. Smithsonian Institute Press, Washington and London.

TYACK, P.L. 2000. Functional aspects of cetacean communication. Pages 270-307 in J. Mann, R. Connor, P. L., Tyack and H. Whitehead, eds. Cetacean Societies: Field studies of dolphins and whales. The University of Chicago Press, Chicago.

TYACK, P., AND WHITEHEAD, H. 1983. Male competition in large groups of wintering humpback whales. Behaviour 83:132-154.

VAn PARIJs, S. M. AND P. J. CORKERON. 2001. Vocalizations and Behavior of Pacific Humpback dolphins Sousa chinensis. Ethology 107:701-716.

VAn PARIJS, S.M., PARRA, G.J. AND P.J. CORKERON. 2000. Sounds produced by Australian Irrawaddy dolphins, Orcaella brevirostris. Journal of the Acoustical Society of America 108:1938-1940

VAN WAerebeeK, K. AND B. WÜrsig. 2002. Pacific white-sided dolphin and dusky dolphins. Pages 859-861 in W.F. Perrin, B. Wursig and J.G.M. Thewissen, eds. Encyclopedia of Marine Mammals Academic Press, San Diego.

Wang, D., Wursig, B., AND Evans, W.E. 1995a. Comparisons of whistles among seven odontocete species. Pages 299-323 in R.A. Kastelien, J.A. Thomas, and P.E. Nachtigal, eds. In Sensory Systems of Aquatic Mammals. DeSpil, Woerden, The Netherlands.

WAng, D., Wursig, B., AND Evans, W. E. 1995b. Whistles of bottlenose dolphins: comparisons among populations. Aquatic Mammals 21:65-77.

Wang, D., Wang, K., Akamatsu, T., And Fujita, F. 1999. Study on whistles of the Chinese River Dolphin or baiji Lipotes vexillifer. Oceanologia et Limonologia Sinica 30:349-354. 
WANG, D., Wursig, B., AND S. LEATHERWWOD. 2001. Whistles of boto, Inia geoffrensis, and tucuxi, Sotalia fluviatilis. Journal of the Acoustical Society of America 109:407-414.

Wang, X., Wang, D., Akamatsu, T., FujtTA, K. And Shiraki, R. 2006. Estimated detection distance of a baiji's (Chinese river dolphin, Lipotes vexillifer) whistles using a passive acoustic survey method. Journal of the Acoustical Society of America 120: 13611365.

WAtKIns, W. A.1987. The $20 \mathrm{~Hz}$ signals of finback whales (Balaenoptera physalus). Journal of the Acoustical Society of America. 82:1901-1912.

WATKIns, W. A. AND D. WARTZOK. 1985. Sensory biophysics of marine mammals. Marine Mammal Science. 1: 219-260.

WAtKIns, W. A., SChEVILl, W. E., AND C. RAY. 1970. Underwater sounds of Monodon (Narwhal). Journal of the Acoustical Society of America 49:595-599.

WATKins, W.A., Daher, M.A., Fristrup, K.M., AND G. NotARbartolo Di Sciara. 1994. Fishing and acoustic behavior of Fraser's dolphin (Lagenodelphis hosei) near Dominica, southeast Caribbean. Caribbean Journal of Science 30:76-82.

Watkins, W. A., Daher, M. A., SAmuels, A., And D. P. Gannon. 1997. Observations of Peponocephala electra, the Melon-headed whale, in the southeastern Caribbean. Caribbean Journal of Science 33:34-40.

Watkins, W. A., DAher, M. A., George, J. E., AND D. Rodriguez. 2004. Twelve years of tracking 52-Hz whale calls from a unique source in the North Pacific. Deep-Sea Research I 51:1889-1901.

WATwOOD S.L., TYACK P.L., AND R.S. Wells. 2004. Whistle sharing in paired male bottlenose dolphins, Tursiops truncatus. Behavioral Ecology and Sociobiology 55:531543.

WILEY, R. H. 1991. Associations of song properties with habitats for territorial oscine birds of eastern North America. American Naturalist 138:973-993.

WILEY, R.H., AND D.G. RICHARDS. 1978. Physical constraints on acoustic communication in the atmosphere: implications for the evolution of animal vocalizations. Behavioral Ecology Sociobiology 3:69-94.

YIN, S. E. 1999. Movement patterns, behaviors, and whistle sounds of dolphin groups off Kaikoura, New Zealand. M.Sc. Thesis. Texas A\&M University, 107 pp. 
Zbinden, K., Pilleri, G., Kraus, C., And O. Bernath. 1977. Observations on the behaviour and underwater sounds of the plumbeous dolphin (Sousa chinensis G. Cuvier 1829 ) in the Indus Delta region. Pages 259-286 in G. Pilleri, ed. Investigations on Cetacea, University of Bern. 
Table 6. Review of published data on Cetacean body size and standard tonal sound frequency variables.

\begin{tabular}{|c|c|c|c|c|c|c|c|c|c|}
\hline \multirow[t]{2}{*}{ Species } & \multicolumn{2}{|c|}{$\begin{array}{l}\text { Maximum } \\
\text { Body Size }\end{array}$} & \multirow[t]{2}{*}{ References } & \multicolumn{5}{|c|}{ Whistle Frequency Variables (kHz) } & \multirow[t]{2}{*}{ References } \\
\hline & $\mathbf{m}$ & Kg & & Max & MMx & Center & Min & MMin & \\
\hline \multicolumn{10}{|l|}{ SubOrder Mysticeti } \\
\hline Balaena glacialis & 17.0 & 80,000 & $\begin{array}{l}\text { Bannister 2002; Reidenberg } \\
\text { \& Laitman } 2002\end{array}$ & 11.23 & 3.14 & $?$ & 0.02 & 0.05 & Parks \& Tyack 2005 \\
\hline B. mysticetus & 19.8 & 100,000 & $\begin{array}{l}\text { Bannister 2002; Rugh \& } \\
\text { Shelden } 2002\end{array}$ & $\begin{array}{l}2 \\
1\end{array}$ & $\begin{array}{c}0.165 \\
\mathbf{0 . 3}\end{array}$ & 0.175 & $\begin{array}{l}0.09 \\
\mathbf{0 . 0 2}\end{array}$ & $\begin{array}{l}\mathbf{0 . 0 2} \\
0.05\end{array}$ & $\begin{array}{l}\text { Ljungblad et al. } 1982 \\
\text { Clark \& Johnson } 1984 \\
\text { Matthews et al. } 1999 \\
\end{array}$ \\
\hline Balaenoptera bonaerensis & 10.7 & 13,500 & $\begin{array}{l}\text { Bannister 2002; Reidenberg } \\
\text { \& Laitman } 2002\end{array}$ & $?$ & $?$ & $?$ & $?$ & $?$ & $?$ \\
\hline B. borealis & 18.3 & 25,000 & Reidenberg \& Laitman 2002 & $\begin{array}{c}3.5 \\
1 \\
\end{array}$ & $?$ & 2.5 & $\begin{array}{r}1.5 \\
\sim 0.1 \\
\end{array}$ & $?$ & $\begin{array}{l}\text { Knowlton et al. } 1991 \\
\text { Matthews et al. } 1999 \\
\text { McDonald et al. } 2005\end{array}$ \\
\hline B. edeni & 15.6 & 25,000 & Reidenberg \& Laitman 2002 & $\begin{array}{l}\mathbf{0 . 2 4 5} \\
0.180 \\
0.079\end{array}$ & & 0.128 & $\begin{array}{c}0.07 \\
0.09 \\
\mathbf{0 . 0 2 0 7}\end{array}$ & & $\begin{array}{l}\text { Cummings et al. } 1986 \\
\text { Edds } 1993 \\
\text { Heimlich et al. } 2005 \\
\text { Matthews et al. } 1999\end{array}$ \\
\hline B. musculus & 31 & 200,000 & Reidenberg \& Laitman 2002 & $\begin{array}{c}0.0185 \\
0.0202 \\
0.122 \\
\\
0.052 \\
0.0277 \\
\\
\mathbf{0 . 2 4 2} \\
\end{array}$ & $\begin{array}{l}0.0189 \\
\mathbf{0 . 0 8 8 3} \\
\end{array}$ & 0.020 & $\begin{array}{c}0.0157 \\
0.0182 \\
- \\
\mathbf{0 . 0 1 1} \\
0.050 \\
0.0189 \\
\\
0.0143 \\
\end{array}$ & $\begin{array}{l}0.0172 \\
\mathbf{0 . 0 1 6 6} \\
\end{array}$ & $\begin{array}{l}\text { Mellinger \& Clark } 2003 \\
\text { Stafford et al. } 2001 \\
\text { Alling } 2003 \text { pers. comm. to } \\
\text { Mellinger \& Clark } 2003 \\
\text { Ljungblad et al. } 1997 \\
\text { Watkins et al. } 2004 \\
\text { Sirovic et al. } 2004 \\
\text { Stafford et al. } 1994 \\
\text { Matthews } \text { et al. } 1999 \\
\text { Berchok et al. } 2006\end{array}$ \\
\hline B. physalus & 27 & 90,000 & Reidenberg \& Laitman 2002 & $\begin{array}{l}\mathbf{0 . 1 1 8} \\
0.058\end{array}$ & $\begin{array}{l}0.023 \\
\mathbf{0 . 0 4 2}\end{array}$ & 0.062 & $\begin{array}{l}\mathbf{0 . 0 1 0} \\
0.017\end{array}$ & $\begin{array}{l}\mathbf{0 . 0 1 8} \\
0.020\end{array}$ & $\begin{array}{l}\text { Edds } 1988 \\
\text { Watkins } 1987 \\
\text { Thompson \& Friedl } 1982 \\
\text { Matthews et al. } 1999\end{array}$ \\
\hline Eschrichtius robustus & 15.2 & 35,000 & Jones \& Swartz 2002 & $\begin{array}{l}\mathbf{0 . 2} \\
0.2\end{array}$ & 0.3 & 0.150 & $\begin{array}{c}\mathbf{0 . 0 2} \\
0.1\end{array}$ & 0.25 & $\begin{array}{l}\text { Cummings et al. } 1968 \\
\text { Fish et al. } 1974 \\
\text { Dahlheim et al. } 1984 \\
\text { Matthews et al. } 1999 \\
\end{array}$ \\
\hline Megaptera novaeangliae & 19.0 & 48,000 & Reidenberg \& Laitman 2002 & 4 & 1.315 & & 0.25 & 0.925 & Hafner et al. 1979 \\
\hline
\end{tabular}




\begin{tabular}{|c|c|c|c|c|c|c|c|c|c|}
\hline & & & & & & 1.108 & & & Matthews et al. 1999 \\
\hline Capera marginata & 6.45 & 3,500 & $\begin{array}{l}\text { Bannister 2002; Reidenberg } \\
\text { \& Laitman 2002 }\end{array}$ & 0.135 & $?$ & 0.07 & 0.06 & $?$ & $\begin{array}{l}\text { Dawbin \& Cato } 1992 \\
\text { Matthews et al. } 1999\end{array}$ \\
\hline \multicolumn{10}{|l|}{ SubOrder Odontoceti } \\
\hline \multicolumn{10}{|c|}{ Kogiidae } \\
\hline Kogia breviceps & 3.7 & 400 & Reidenberg \& Laitman 2002 & $?$ & ? & ? & ? & $?$ & \\
\hline K. simus (sima) & 2.7 & 210 & Reidenberg \& Laitman 2002 & $?$ & $?$ & $?$ & $?$ & $?$ & \\
\hline \multicolumn{10}{|c|}{ Physeteridae } \\
\hline Physeter macrocephalus & 20.5 & 57,000 & Reidenberg \& Laitman 2002 & $\mathrm{n} / \mathrm{a}$ & $\mathrm{n} / \mathrm{a}$ & $\mathrm{n} / \mathrm{a}$ & $\mathrm{n} / \mathrm{a}$ & $\mathrm{n} / \mathrm{a}$ & \\
\hline \multicolumn{10}{|c|}{ Ziphiidae } \\
\hline $\begin{array}{l}\text { Berardius bairdii (B. } \\
\text { anurxii) }\end{array}$ & 12.8 & 11,000 & Reidenberg \& Laitman 2002 & 8.0 & $?$ & 6.0 & 4 & $?$ & $\begin{array}{l}\text { Dawson \& Barlow } 1998 \\
\text { Matthews et al. 1999, }\end{array}$ \\
\hline Hyperoodon ampullatus & 9.8 & $?$ & Reidenberg \& Laitman 2002 & $\mathrm{n} / \mathrm{a}$ & $\mathrm{n} / \mathrm{a}$ & $\mathrm{n} / \mathrm{a}$ & $\mathrm{n} / \mathrm{a}$ & $\mathrm{n} / \mathrm{a}$ & \\
\hline H. planifrons & 7.45 & $?$ & Reidenberg \& Laitman 2002 & $?$ & $?$ & $?$ & $?$ & $?$ & \\
\hline Mesoplodon bidens & 5.5 & $?$ & $\begin{array}{l}\text { Pitman 2002a; Reidenberg \& } \\
\text { Laitman 2002 }\end{array}$ & $?$ & $?$ & $?$ & $?$ & $?$ & \\
\hline M. densirostris & 4.73 & 1,033 & $\begin{array}{l}\text { Pitman 2002a; Reidenberg \& } \\
\text { Laitman } 2002\end{array}$ & $?$ & $?$ & $?$ & $?$ & $?$ & \\
\hline Ziphius cavirostris & 7.5 & 3,000 & Reidenberg \& Laitman 2002 & $?$ & $?$ & $?$ & $?$ & $?$ & \\
\hline Tasmacetus shepherdi & 7.0 & $?$ & Mead 2002 & $?$ & $?$ & $?$ & $?$ & $?$ & \\
\hline Indopacetus pacificus & 8.0 & $?$ & Pitman 2002b & $?$ & $?$ & $?$ & $?$ & $?$ & \\
\hline \multicolumn{10}{|c|}{$\begin{array}{ll}\text { Platanistidae } \\
\end{array}$} \\
\hline Platanista gangetica & 2.5 & 85 & Reidenberg \& Laitman 2002 & $?$ & $?$ & $?$ & $?$ & $?$ & \\
\hline \multicolumn{10}{|c|}{ Iniidae } \\
\hline Inia geoffrensis & 2.6 & 160 & $\begin{array}{l}\text { Da Silva 2002; Reidenberg \& } \\
\text { Laitman } 2002\end{array}$ & $\begin{array}{c}48.10 \\
5.16 \\
13\end{array}$ & $\begin{array}{l}24.71 \\
2.97\end{array}$ & 2.75 & $\begin{array}{c}5.03 \\
0.22 \\
3\end{array}$ & $\begin{array}{l}15.06 \\
\mathbf{2 . 5 4}\end{array}$ & $\begin{array}{l}\text { May-Collado \& Wartzok } \\
2007 \\
\text { Matthews et al. } 1999, \\
\text { Wang et al. 1995a, 2001 } \\
\text { Diazgranados \& Trujillo } 2002 \\
\end{array}$ \\
\hline \multicolumn{10}{|c|}{ Lipotidae } \\
\hline Lipotes vexillifer & 2.53 & 160 & $\begin{array}{l}\text { Kaiya 2002; Reidenberg \& } \\
\text { Laitman } 2002\end{array}$ & $\begin{array}{l}4.5 \\
4.6\end{array}$ & $\begin{array}{c}5.84 \\
6.1\end{array}$ & 6.0 & $\begin{array}{l}3.8 \\
3.8 \\
\end{array}$ & $\begin{array}{c}4.975 \\
\mathbf{4 . 9}\end{array}$ & $\begin{array}{l}\text { Matthews et al. } 1999 \text {, } \\
\text { Wang et al. } 1999 \\
\text { Wang et al. } 2006\end{array}$ \\
\hline \multicolumn{10}{|c|}{ Pontoporidae } \\
\hline Pontoporia blainvillei & 1.77 & 53 & Crespo 2002 & $?$ & $?$ & $?$ & $?$ & $?$ & \\
\hline \multicolumn{10}{|c|}{ Super Family Delphonidea } \\
\hline \multicolumn{10}{|c|}{$\begin{array}{ll} & \text { Phocoenidae } \\
\end{array}$} \\
\hline $\begin{array}{l}\text { Australophocaena } \\
\text { dioptrica* }\end{array}$ & 2.4 & 115 & $\begin{array}{l}\text { Reidenberg \& Laitman 2002; } \\
\text { Goodall 2002a }\end{array}$ & $\mathrm{n} / \mathrm{a}$ & $\mathrm{n} / \mathrm{a}$ & $\mathrm{n} / \mathrm{a}$ & $\mathrm{n} / \mathrm{a}$ & $\mathrm{n} / \mathrm{a}$ & \\
\hline Phocoena phocoena & 2.0 & 90 & Bjøge et al. 2002; Reidenberg & $\mathrm{n} / \mathrm{a}$ & $\mathrm{n} / \mathrm{a}$ & $\mathrm{n} / \mathrm{a}$ & $\mathrm{n} / \mathrm{a}$ & $\mathrm{n} / \mathrm{a}$ & \\
\hline
\end{tabular}




\begin{tabular}{|c|c|c|c|c|c|c|c|c|c|}
\hline & & & \& Laitman 2002 & & & & & & \\
\hline P. sinus & 1.4 & $?$ & $\begin{array}{l}\text { Rojas-Bracho \& Jaramillo- } \\
\text { Legorreta } 2002\end{array}$ & $\mathrm{n} / \mathrm{a}$ & $\mathrm{n} / \mathrm{a}$ & $\mathrm{n} / \mathrm{a}$ & $\mathrm{n} / \mathrm{a}$ & $\mathrm{n} / \mathrm{a}$ & \\
\hline P. spinipinnis & 2.0 & $?$ & Reyes 2002 & $\mathrm{n} / \mathrm{a}$ & $\mathrm{n} / \mathrm{a}$ & $\mathrm{n} / \mathrm{a}$ & $\mathrm{n} / \mathrm{a}$ & $\mathrm{n} / \mathrm{a}$ & \\
\hline Phocoenoides dalli & 2.39 & 200 & Jefferson $2002 \mathrm{a}$ & $\mathrm{n} / \mathrm{a}$ & $\mathrm{n} / \mathrm{a}$ & $\mathrm{n} / \mathrm{a}$ & $\mathrm{n} / \mathrm{a}$ & $\mathrm{n} / \mathrm{a}$ & \\
\hline $\begin{array}{l}\text { Neophocaena } \\
\text { phocaenoides }\end{array}$ & 1.9 & 100 & $\begin{array}{l}\text { Reidenberg \& Laitman 2002; } \\
\text { Amano } 2002\end{array}$ & $\mathrm{n} / \mathrm{a}$ & $\mathrm{n} / \mathrm{a}$ & $\mathrm{n} / \mathrm{a}$ & $\mathrm{n} / \mathrm{a}$ & $\mathrm{n} / \mathrm{a}$ & \\
\hline \multicolumn{10}{|c|}{$\begin{array}{ll}\text { Monodontidae } \\
\end{array}$} \\
\hline Monodon monocerus & 4.7 & 1600 & $\begin{array}{l}\text { Heide-Jørgensen 2002; } \\
\text { Reidenberg \& Laitman } 2002\end{array}$ & $\begin{array}{r}\mathbf{1 8} \\
10 \\
8.84\end{array}$ & 7.18 & 5.2 & $\begin{array}{c}\mathbf{0 . 3} \\
5 \\
0.360\end{array}$ & 0.718 & $\begin{array}{l}\text { Ford \& Fisher } 1978, \\
\text { Watkins et al. } 1970 \\
\text { Matthews } \text { et al. } 1999 \\
\text { Shapiro } 2006\end{array}$ \\
\hline Delphinapterus leucas & 5.5 & 1500 & $\begin{array}{l}\text { O'Corry-Crowe 2002; } \\
\text { Reidenberg \& Laitman } 2002\end{array}$ & $\begin{array}{l}\mathbf{1 9 . 6} \\
15.8\end{array}$ & $\begin{array}{c}6.8 \\
4.33 \\
\mathbf{1 1 . 6 5}\end{array}$ & 3.89 & $\begin{array}{l}\mathbf{0 . 2} \\
0.4\end{array}$ & $\begin{array}{c}3.8 \\
3.38 \\
\mathbf{1 . 9 9}\end{array}$ & $\begin{array}{l}\text { Matthews et al. } 1999 \text {, } \\
\text { Karlsen } \text { et al. } 2002 ; \\
\text { Sjare \& Smith } 1986 \\
\text { Belikov \& Bel'kovitch } 2001\end{array}$ \\
\hline \multicolumn{10}{|c|}{ Delphinidae } \\
\hline $\begin{array}{l}\text { Cephalorhynchus } \\
\text { commersonii }\end{array}$ & 1.75 & 86 & $\begin{array}{l}\text { Dawson 2002; Reidenberg \& } \\
\text { Laitman } 2002\end{array}$ & $\mathrm{n} / \mathrm{a}$ & $\mathrm{n} / \mathrm{a}$ & $\mathrm{n} / \mathrm{a}$ & $\mathrm{n} / \mathrm{a}$ & $\mathrm{n} / \mathrm{a}$ & \\
\hline C. eutropia & 1.67 & 63 & Dawson 2002 & $\mathrm{n} / \mathrm{a}$ & $\mathrm{n} / \mathrm{a}$ & $\mathrm{n} / \mathrm{a}$ & $\mathrm{n} / \mathrm{a}$ & $\mathrm{n} / \mathrm{a}$ & \\
\hline C. hectori & 1.8 & 60 & Reidenberg \& Laitman 2002 & $\mathrm{n} / \mathrm{a}$ & $\mathrm{n} / \mathrm{a}$ & $\mathrm{n} / \mathrm{a}$ & $\mathrm{n} / \mathrm{a}$ & $\mathrm{n} / \mathrm{a}$ & \\
\hline C. heavisidii & 1.74 & 75 & Dawson 2002 & $\mathrm{n} / \mathrm{a}$ & $\mathrm{n} / \mathrm{a}$ & $\mathrm{n} / \mathrm{a}$ & $\mathrm{n} / \mathrm{a}$ & $\mathrm{n} / \mathrm{a}$ & \\
\hline Lagenorhynchus australis & 2.18 & 115 & Goodall 2002b & $\mathrm{n} / \mathrm{a}$ & $\mathrm{n} / \mathrm{a}$ & $\mathrm{n} / \mathrm{a}$ & $\mathrm{n} / \mathrm{a}$ & $\mathrm{n} / \mathrm{a}$ & \\
\hline L.cruciger & 1.87 & 88 & Goodall 2002c & $?$ & $?$ & $?$ & $?$ & $?$ & \\
\hline L. obliquidens & 2.5 & 181 & $\begin{array}{l}\text { Van Waerebeek \& Würsig } \\
\text { 2002; Reidenberg \& Laitman } \\
2002\end{array}$ & $?$ & $?$ & $?$ & $?$ & $?$ & \\
\hline L. obscurus & 2.1 & 80 & $\begin{array}{l}\text { Van Waerebeek \& Würsig } \\
\text { 2002; Reidenberg \& Laitman } \\
\text { 2002 }\end{array}$ & 27.3 & $\begin{array}{l}\mathbf{1 6 . 4 9} \\
13.22 \\
\end{array}$ & 12.4 & 1.04 & $\begin{array}{l}8.11 \\
8.15 \\
\end{array}$ & $\begin{array}{l}\text { Wang et al. } 1995 \mathrm{a} \\
\text { Matthews et al. } 1999 \\
\text { Yin } 1999\end{array}$ \\
\hline L. acutus & 2.7 & 230 & $\begin{array}{l}\text { Cipriano 2002; Reidenberg \& } \\
\text { Laitman 2002 }\end{array}$ & $?$ & 12.14 & 10.37 & $?$ & 8.21 & $\begin{array}{l}\text { Steiner } 1981 \\
\text { Matthews et al. } 1999\end{array}$ \\
\hline Lissodelphins borealis & 3.1 & 116 & $\begin{array}{l}\text { Reidenberg \& Laitman 2002; } \\
\text { Lipsky } 2002\end{array}$ & $?$ & $?$ & $?$ & $?$ & $?$ & \\
\hline L. peronii & 3.0 & 116 & Lipsky 2002 & $?$ & $?$ & $?$ & $?$ & $?$ & \\
\hline Delphinus delphis & 2.35 & 200 & Perrin 2002a & 19.8 & $\begin{array}{l}11.65 \\
\mathbf{1 3 . 6}\end{array}$ & 8.81 & 4.8 & $\begin{array}{l}6.42 \\
7.4\end{array}$ & $\begin{array}{l}\text { Moore \& Ridgway } 1995 \\
\text { Matthews et al. } 1999, \\
\text { Oswald et al. } 2003\end{array}$ \\
\hline D. capensis & 2.35 & 235 & Perrin 2002a & $?$ & 15.5 & $?$ & $?$ & 7.7 & Oswald et al. 2003 \\
\hline Stenella attenuata & 2.57 & 119 & Perrin 2002b; Reidenberg \& & 21.4 & 15.72 & & 3.13 & 8.73 & Wang et al. $1995 \mathrm{a}$ \\
\hline
\end{tabular}




\begin{tabular}{|c|c|c|c|c|c|c|c|c|c|}
\hline & & & Laitman 2002 & & 18.7 & 12.54 & & 8.2 & $\begin{array}{l}\text { Matthews et al. } 1999 \\
\text { Oswald et al. } 2003\end{array}$ \\
\hline S. clymene & 2.0 & 80 & $\begin{array}{l}\text { Jefferson } 2002 b \text {, Jefferson \& } \\
\text { Curry } 2003\end{array}$ & $\begin{array}{c}\mathbf{1 9 . 2} \\
13.62\end{array}$ & $?$ & 11.66 & $?$ & $\begin{array}{l}6.33 \\
9.25\end{array}$ & $\begin{array}{l}\text { Watkins \& Wartzok } 1985 \\
\text { Mullin et al. } 1994 \\
\text { Matthews et al. } 1999 \\
\end{array}$ \\
\hline S. frontalis & 2.3 & 143 & $\begin{array}{l}\text { Perrin 2002c; Reidenberg \& } \\
\text { Laitman } 2002\end{array}$ & 19.8 & 16.04 & 11.62 & 5 & 7.91 & $\begin{array}{l}\text { Wang et al. } 1995 \mathrm{a} \\
\text { Matthews et al. } 1999\end{array}$ \\
\hline S. coeruleoalba & 2.4 & 156 & Reidenberg \& Laitman 2002 & 22.99 & $\begin{array}{c}11.53 \\
14.8\end{array}$ & 9.07 & 1.1 & $\begin{array}{c}6.84 \\
8.1\end{array}$ & $\begin{array}{l}\text { Matthews et al. } 1999 \text {, } \\
\text { Oswald et al. } 2003\end{array}$ \\
\hline S. longirostris & 2.16 & 75 & $\begin{array}{l}\text { Perrin 2002d; Reidenberg \& } \\
\text { Laitman } 2002\end{array}$ & $\begin{array}{c}22.5 \\
\\
24 \\
\mathbf{2 5 . 2 5} \\
23.04\end{array}$ & \begin{tabular}{c|}
15.2 \\
\\
13.7 \\
16.5 \\
$\mathbf{1 7 . 5 6}$ \\
16.8 \\
14.32 \\
\end{tabular} & 12.22 & $\begin{array}{c}3.91 \\
0.85 \\
4\end{array}$ & $\begin{array}{c}9.03 \\
\\
9.1 \\
9.99 \\
9.66 \\
10.19 \\
8.76 \\
\end{array}$ & $\begin{array}{l}\text { Wang et al. } 1995 \mathrm{a} \\
\text { Matthews et al. 1999; } \\
\text { Oswald et al. 2003; } \\
\text { Barzua-Duran \& Au 2002, } \\
\text { Barzua-Duran \& Au 2004 } \\
\text { Driscoll 1995 } \\
\text { Steiger } 1981\end{array}$ \\
\hline Tursiops truncatus & 4.0 & 650 & Reidenberg \& Laitman 2002; & $\begin{array}{r}41 \\
21.6\end{array}$ & $\begin{array}{c}\mathbf{1 7 . 2} \\
11.35 / 11.95 \\
16.24 \\
\end{array}$ & 8.09 & $1.86 / \mathbf{0 . 9 4}$ & $\begin{array}{c}7.4 \\
\mathbf{5 . 4 6} \\
7.33 \\
\end{array}$ & $\begin{array}{l}\text { Boisseau } 2005 \\
\text { Matthews et al. 1999; Oswald } \\
\text { et al. 2003; } \\
\text { Wang et al. 1995a,b; } \\
\text { Steiner } 1981\end{array}$ \\
\hline Lagenodelphis hosei & 2.65 & 200 & $\begin{array}{l}\text { Dolar 2002; Reidenberg \& } \\
\text { Laitman } 2002\end{array}$ & $\begin{array}{l}24.0 \\
13.4 \\
\end{array}$ & $\begin{array}{c}\mathbf{1 6 . 9} \\
16.56\end{array}$ & 12.82 & 4.3 & $\begin{array}{c}9.36 \\
11.949 \\
\mathbf{7 . 6 4} \\
\end{array}$ & $\begin{array}{l}\text { Matthews et al. } 1999 ; \\
\text { Watkins et al. } 1994 \\
\text { Oswald } \text { et al. submitted } \\
\text { Leatherwood } \text { et al. } 1993 \\
\end{array}$ \\
\hline Sousa chinensis & 3 & 284 & $\begin{array}{l}\text { Ross 2002; Reidenberg \& } \\
\text { Laitman } 2002\end{array}$ & $\begin{array}{l}22 \\
20\end{array}$ & 16.3 & $?$ & $\begin{array}{c}\mathbf{0 . 9} \\
3 \\
1.2\end{array}$ & 4.5 & $\begin{array}{l}\text { Van Parijs \& Corkeron } \\
2001 ; \\
\text { Zbinden et al. } 1977 ; \\
\text { Schultz \& Corkeron } 1994\end{array}$ \\
\hline Sotalia fluviatilis** & 2.20 & 40 & Flores 2002 & $\begin{array}{c}23.9 \\
\mathbf{3 8 . 2 5} \\
\\
18 \\
17.49\end{array}$ & $\begin{array}{c}19.95 \\
15.41 \\
\mathbf{2 1 . 3 2} \\
\\
13 \\
13.312 \\
15.65 \\
\end{array}$ & 12.68 & $\begin{array}{c}1.34 \\
3.65 \\
2.714 \\
\\
\mathbf{0 . 5} \\
1.031\end{array}$ & $\begin{array}{c}7.21 \\
10.2 \\
13.14 \\
\\
7.6 \\
10.521 \\
9.18 \\
\end{array}$ & $\begin{array}{l}\text { Matthews et al. 1999; } \\
\text { Azevedo \& van Sluys 2005; } \\
\text { Wang et al. 1995a, 2001; } \\
\text { May-Collado \& Wartzok } \\
\text { unpublished. } \\
\text { Azevedo \& Simao 2002; } \\
\text { Erber \& Simao } 2004 \\
\text { Podos et al. } 2002 \\
\end{array}$ \\
\hline Steno bredanensis & 2.65 & 155 & $\begin{array}{l}\text { Jefferson 2002c; Miyasaki \& } \\
\text { Perrin } 1994\end{array}$ & 7.0 & 9.1 & 5.5 & 4 & 6.03 & $\begin{array}{l}\text { Busnell \& Dziedzic } 1968 \\
\text { Matthews et al. } 1999 ; \\
\text { Oswald } \text { et al. 2003; }\end{array}$ \\
\hline Feresa attenuata & 2.7 & 225 & Reidenberg \& Laitman 2002 & $?$ & $?$ & $?$ & $?$ & $?$ & \\
\hline
\end{tabular}




\begin{tabular}{|c|c|c|c|c|c|c|c|c|c|}
\hline $\begin{array}{l}\text { Globicephala } \\
\text { macrorhynchus }\end{array}$ & 7.2 & 3950 & Reidenberg \& Laitman 2002 & 23.6 & $\begin{array}{c}10.87 \\
6.1 \\
\end{array}$ & 7.87 & 0.24 & $\begin{array}{l}6.25 \\
\mathbf{3 . 6}\end{array}$ & $\begin{array}{l}\text { Matthews et al. } 1999 ; \\
\text { Rendell et al. } 1999 ; \\
\text { Oswald } \text { et al. } 2003\end{array}$ \\
\hline G. melas & 6.3 & 1750 & Reidenberg \& Laitman 2002 & 21.2 & $\begin{array}{c}\mathbf{8 . 8 6} \\
4.716\end{array}$ & 4.48 & 0.32 & $\begin{array}{l}3.48 \\
\mathbf{2 . 8 2}\end{array}$ & $\begin{array}{l}\text { Matthews et al. 1999; } \\
\text { Rendell et al. 1999; } \\
\text { Steiner } 1981\end{array}$ \\
\hline Grampus griseus & 4.3 & 500 & Reidenberg \& Laitman 2002 & 23.8 & $\begin{array}{c}\mathbf{2 0} \\
13.44 \\
\end{array}$ & 11.3 & 1.90 & $\begin{array}{c}3.9 \\
8.83\end{array}$ & $\begin{array}{l}\text { Matthews et al. } 1999 ; \\
\text { Corkeron \& Van Parijs 2001; } \\
\text { Rendell et al. } 1999\end{array}$ \\
\hline Peponocephala electra & 2.75 & 275 & Perryman 2002 & 24.5 & 12.14 & 12.75 & 5.5 & 8.381 & $\begin{array}{l}\text { Matthews et al. } 1999 \text {, } \\
\text { Oswald } \text { et al. submitted, } \\
\text { Watkins } \text { et al. } 1997\end{array}$ \\
\hline Pseudorca crassidens & 6 & 2200 & $\begin{array}{l}\text { Baird 2002; Reidenberg \& } \\
\text { Laitman 2002 }\end{array}$ & 18.1 & $\begin{array}{c}8.29 \\
6.1 \\
\end{array}$ & 6.82 & 1.87 & $\begin{array}{c}5.43 \\
4.7\end{array}$ & $\begin{array}{l}\text { Matthews et al. } 1999 ; \\
\text { Rendell et al. } 1999 ; \\
\text { Oswald } \text { et al. } 2003\end{array}$ \\
\hline Orcaella brevirostris $* *$ & 2.75 & 150 & Reidenberg \& Laitman 2002 & 6.0 & 4.2 & $?$ & 1.1 & 3.2 & Van Parijs et al. 2000 \\
\hline Orcinus orca & 9.75 & 10,500 & Reidenberg \& Laitman 2002 & $\begin{array}{c}\mathbf{1 8} \\
\\
\\
8.9 \\
16.7 \\
\end{array}$ & $\begin{array}{c}6.61 \\
\\
\\
9.9 \\
\mathbf{1 2 . 6 4} \\
\end{array}$ & 5.0 & $\begin{array}{c}\mathbf{0 . 0 5} \\
2.4\end{array}$ & $\begin{array}{c}\mathbf{1 . 5} \\
4.27 \\
\\
\\
\\
5.4 \\
3.36 \\
\end{array}$ & $\begin{array}{l}\text { Ford 1989; } \\
\text { Dahleim \& Awbrey 1982; } \\
\text { Matthews et al. } 1999 \\
\text { Steiner } \text { et al. } 1979 \\
\text { Thomsem } \text { et al. } 2001 \\
\text { Riesch } \text { et al. } 2006\end{array}$ \\
\hline
\end{tabular}

*Now recognized as Phocoena dioptrica (Lahille 1912)

**In this paper these species are still treated as one single species (with two ecotypes: riverine and marine), however there is recent evidence that each may be a separate species (see details in Cunha et al. 2005 and Beasley et al. 2005) 
Table 7. Previous and present regression analyses between body size and standard tonal sound frequency variables. This study uses independent contrast both on the entire (values in parenthesis) and pruned (values in bold) data sets (see Methods).

\begin{tabular}{|c|c|c|c|c|c|}
\hline Source & Maximum & Mean Maximum & $\begin{array}{l}\text { Minimum } \\
\end{array}$ & Mean Minimum & Center \\
\hline $\begin{array}{l}\text { Wang et al. } 1995 \\
\text { Delphinids + Inia } \\
\text { R-square } \\
\text { d.f. } \\
\text { p-value }\end{array}$ & $\begin{array}{c}79 \%, \\
97 \% \text { (excluding Inia) } \\
9 \\
<0.05^{* *}\end{array}$ & --.-- & --.-- & --.-- & ---- \\
\hline $\begin{array}{c}\text { Matthews et al. } 1999 \\
\text { Toothed-whales } \\
\text { R-square } \\
\text { d.f. } \\
\text { p-value }\end{array}$ & $\begin{array}{c}68 \% \text { (uwtd.), } 76 \% \text { (wtd.) } \\
18,16 \\
<0.001,<0.001^{* *}\end{array}$ & ...--_-.. & $\begin{array}{c}86 \% \text { (uwtd.), } 93 \% \text { (wtd.) } \\
18,16 \\
<0.001,<0.001^{* *}\end{array}$ & ..--..- & $\begin{array}{c}69 \% \text { (uwtd.), } 84 \% \text { (wtd.) } \\
25,14 \\
<0.001,<0.001^{* *}\end{array}$ \\
\hline $\begin{array}{c}\text { Matthews et al. } 1999 \\
\text { Baleen-whales } \\
\text { R-square } \\
\text { d.f. } \\
\text { p-value }\end{array}$ & ---- & --.-- & --- & --.- & $\begin{array}{c}64 \% \text { (uwtd.) } \\
9 \\
0.003^{* *}\end{array}$ \\
\hline $\begin{array}{c}\text { Podos et al. } 2002 \\
\text { Delphinids stinia } \\
\text { R-square } \\
\text { d.f. } \\
\text { p-value }\end{array}$ & $\begin{array}{c}85.1 \% \\
16 \\
<0.05^{* *}\end{array}$ & --.-- & 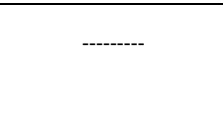 & -- & --.-- \\
\hline $\begin{array}{l}\text { This study Body Length } \\
\text { Cetacean-Tonal Sounds } \\
\text { R-square } \\
\text { d.f. } \\
\text { p-value }\end{array}$ & $\begin{array}{c}\mathbf{0 . 0 9 6 3} \%(0.0406 \%) \\
32 \\
\mathbf{0 . 4 1 3}(0.455) \\
\end{array}$ & $\begin{array}{c}0.18 \%(0.0732 \%) \\
28 \\
0.410(0.443)\end{array}$ & $\begin{array}{c}26.3 \%(22.8 \%) \\
32 \\
0.001(0.002)^{* *}\end{array}$ & $\begin{array}{c}\mathbf{1 2 . 8 \%}(10.2 \%) \\
29 \\
\mathbf{0 . 0 2 4}(0.040)^{* * *}\end{array}$ & $\begin{array}{c}4.2 \%(3.5 \%) \\
28 \\
0.136(0.160)\end{array}$ \\
\hline $\begin{array}{c}\text { Toothed-Whales-Whistle } \\
\text { R-square } \\
\text { d.f. } \\
\text { p-value }\end{array}$ & $\begin{array}{c}\mathbf{2 . 2 \%}(1.45 \%) \\
23 \\
\mathbf{0 . 2 4 1}(0.283)\end{array}$ & $\begin{array}{c}\mathbf{3 . 9} \%(4.5 \%) \\
22 \\
\mathbf{0 . 1 7 4}(0.160)\end{array}$ & $\begin{array}{c}27.7 \%(23 \%) \\
23 \\
0.003(0.008)^{* *}\end{array}$ & $\begin{array}{c}\mathbf{6 . 2} \%(5.7 \%) \\
23 \\
0.116(0.125)\end{array}$ & $\begin{array}{r}\mathbf{1 8 . 2 \%}(14.1 \%) \\
20 \\
0.023\left(0.042^{* *}\right)\end{array}$ \\
\hline $\begin{array}{c}\text { Baleen-whales } \\
\text { R-square } \\
\text { d.f. } \\
\text { p-value } \\
\end{array}$ & $\begin{array}{c}\mathbf{0 . 0 5 2} \%(0.37 \%) \\
7 \\
\mathbf{0 . 4 7 6}(0.437) \\
\end{array}$ & $\begin{array}{c}\mathbf{3 3 . 3} \%(14.2 \%)(!) \\
4 \\
\mathbf{0 . 1 1}(0.230) \\
\end{array}$ & $\begin{array}{c}10.2 \%(7.8 \%) \\
7 \\
0.201(0.233) \\
\end{array}$ & $\begin{array}{c}\mathbf{6 6 . 2 \%}(80.3 \%)(!) \\
4 \\
0.024(0.007)^{* *} \\
\end{array}$ & $\begin{array}{c}\mathbf{0 . 0 0 2 8}(0.496 \%) \\
6 \\
\mathbf{0 . 4 9 5}(0.434) \\
\end{array}$ \\
\hline $\begin{array}{l}\text { This study Body Mass } \\
\text { Cetacean-Tonal Sounds } \\
\text { R-square } \\
\text { d.f. } \\
\text { p-value } \\
\end{array}$ & $\begin{array}{c}\mathbf{0 . 0 0 4 2 7 \%}(0.00463 \%) \\
32 \\
\mathbf{0 . 4 8 5}(0.485) \\
\end{array}$ & $\begin{array}{c}\mathbf{0 . 5 2 0} \%(0.144 \%) \\
28 \\
\mathbf{0 . 4 5 2}(0.421) \\
\end{array}$ & $\begin{array}{r}13.5 \%(12.06 \%) \\
32 \\
0.016(0.022)^{* *} \\
\end{array}$ & $\begin{array}{c}\mathbf{1 0 . 1 \%}(8.2 \%) \\
29 \\
\mathbf{0 . 0 4 0 * * ( 0 . 0 5 8 )} \\
\end{array}$ & $\begin{array}{c}7.4 \%(6.04 \%) \\
28 \\
\mathbf{0 . 0 7 4}(0.095)\end{array}$ \\
\hline $\begin{array}{c}\text { Toothed-Whales-Whistle } \\
\text { R-square } \\
\text { d.f. } \\
\text { p-value }\end{array}$ & $\begin{array}{c}\mathbf{1 . 0 4 \%}(0.52 \%) \\
23 \\
\mathbf{0 . 3 1 3}(0.365)\end{array}$ & $\begin{array}{c}\mathbf{3 . 6 0 \%}(4.10 \%) \\
22 \\
\mathbf{0 . 1 8 6}(0.170)\end{array}$ & $\begin{array}{c}11.1 \%(9.3 \%) \\
23 \\
0.052(0.068)\end{array}$ & $\begin{array}{c}5.2 \%(4.9 \%) \\
23 \\
0.137(0.142)\end{array}$ & $\begin{array}{c}\mathbf{1 6 . 1 \%}(12.2 \%) \\
20 \\
\mathbf{0 . 0 3 3}^{* *(0.055)}\end{array}$ \\
\hline $\begin{array}{l}\text { Baleen-whales } \\
\text { R-square } \\
\text { d.f. } \\
\text { p-value }\end{array}$ & $\begin{array}{c}0.0107(0.21 \%) \\
7 \\
0.489(0.452)\end{array}$ & $\begin{array}{c}24.7 \%(3.2 \%)(!) \\
4 \\
0.158(0.367) \\
\end{array}$ & $\begin{array}{c}\mathbf{1 7 . 4 \%}(16.0 \%) \\
7 \\
\mathbf{0 . 1 3 0}(0.142)\end{array}$ & $\begin{array}{c}\mathbf{6 5 . 5 \%} \%(49.0 \%)(!) \\
4 \\
\mathbf{0 . 0 2 5} *(0.059)\end{array}$ & $\begin{array}{c}\mathbf{3 . 5 \%}(2.10 \%) \\
6 \\
\mathbf{0 . 3 2 6}(0.366)\end{array}$ \\
\hline
\end{tabular}

** Significant results (based on the level of significance of $\mathrm{p} \leq 0.05$ )

(!) very small number of contrasts 
FIG. 7

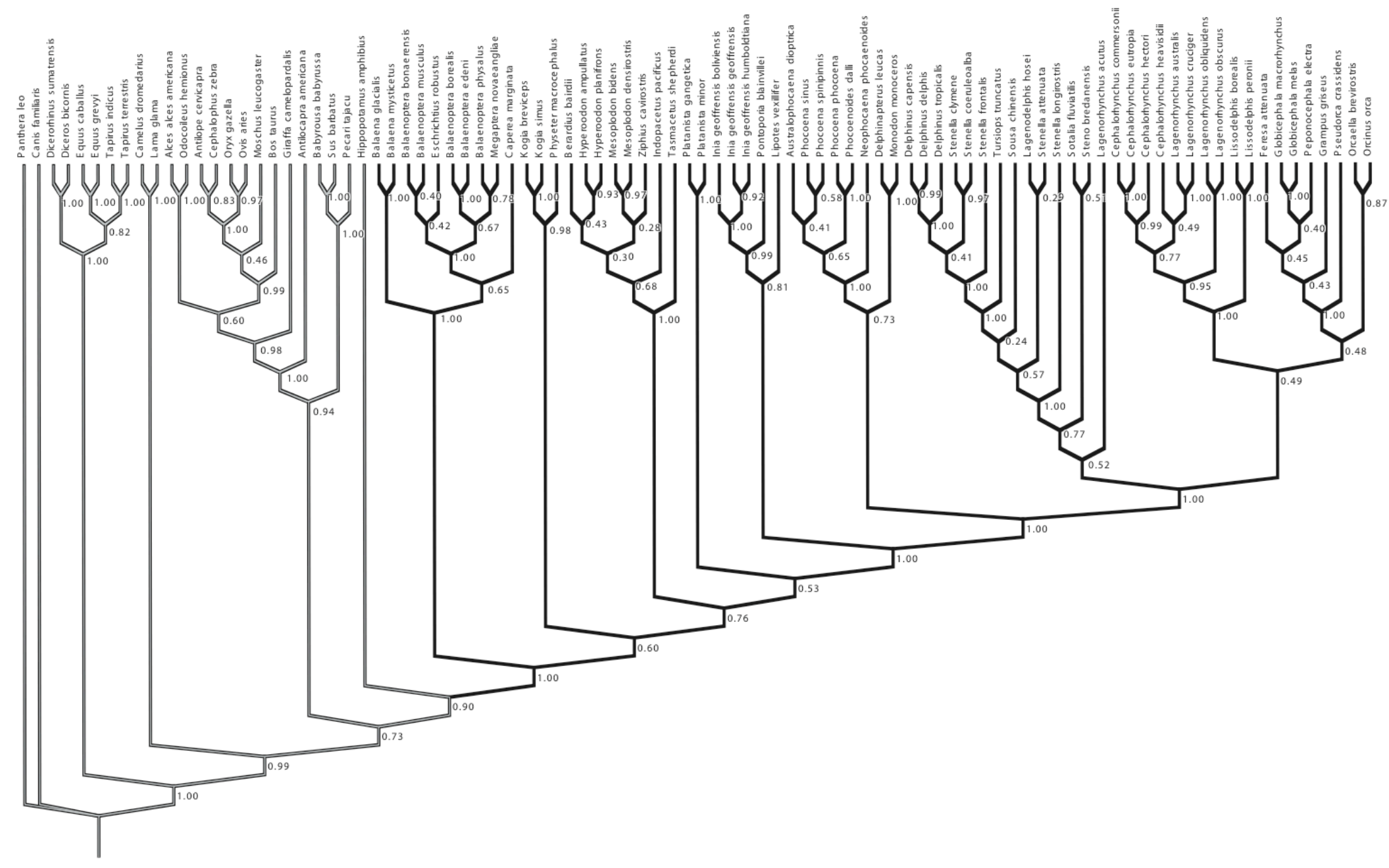


FIG. 8

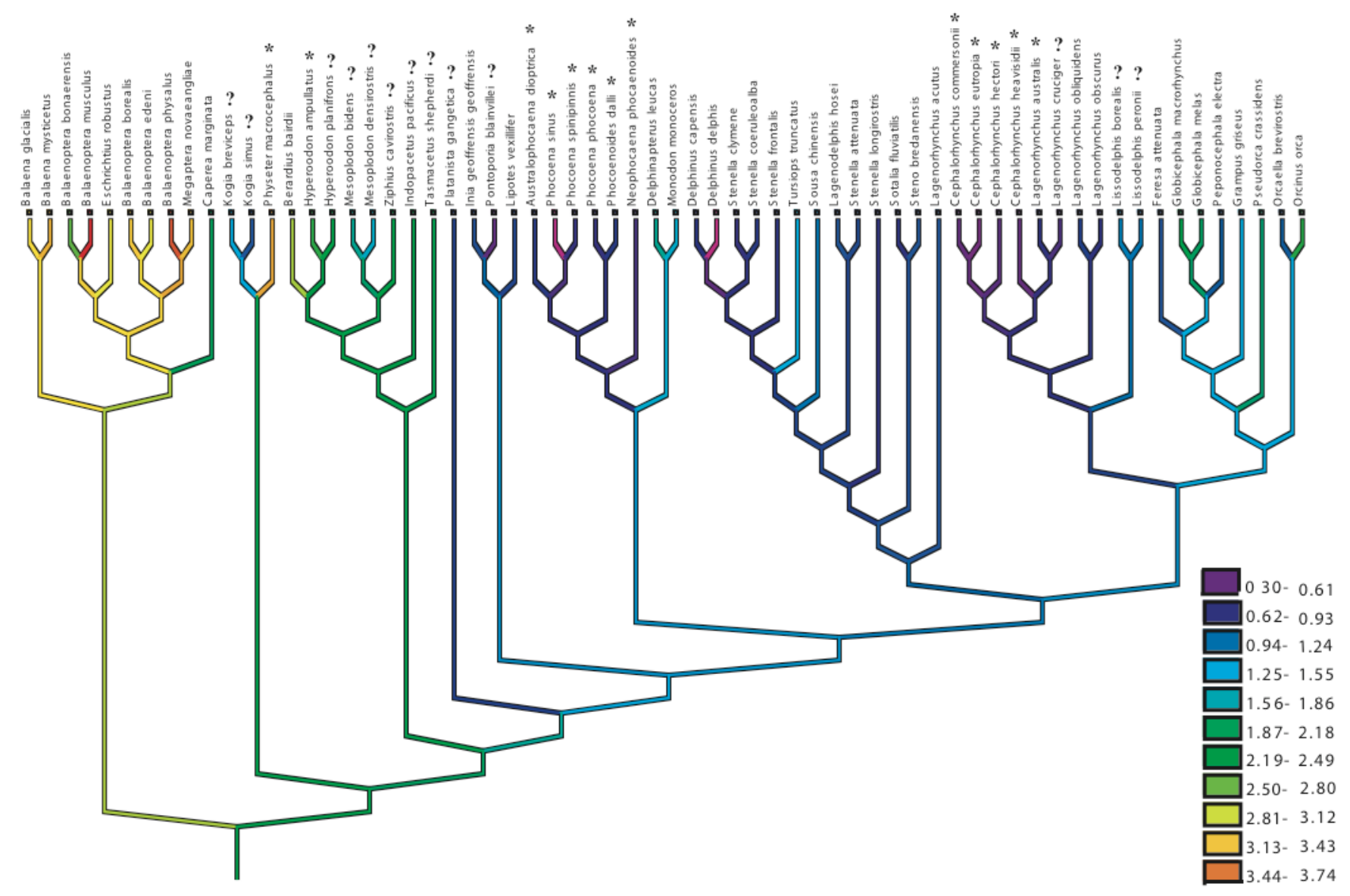




\section{FIG.9}
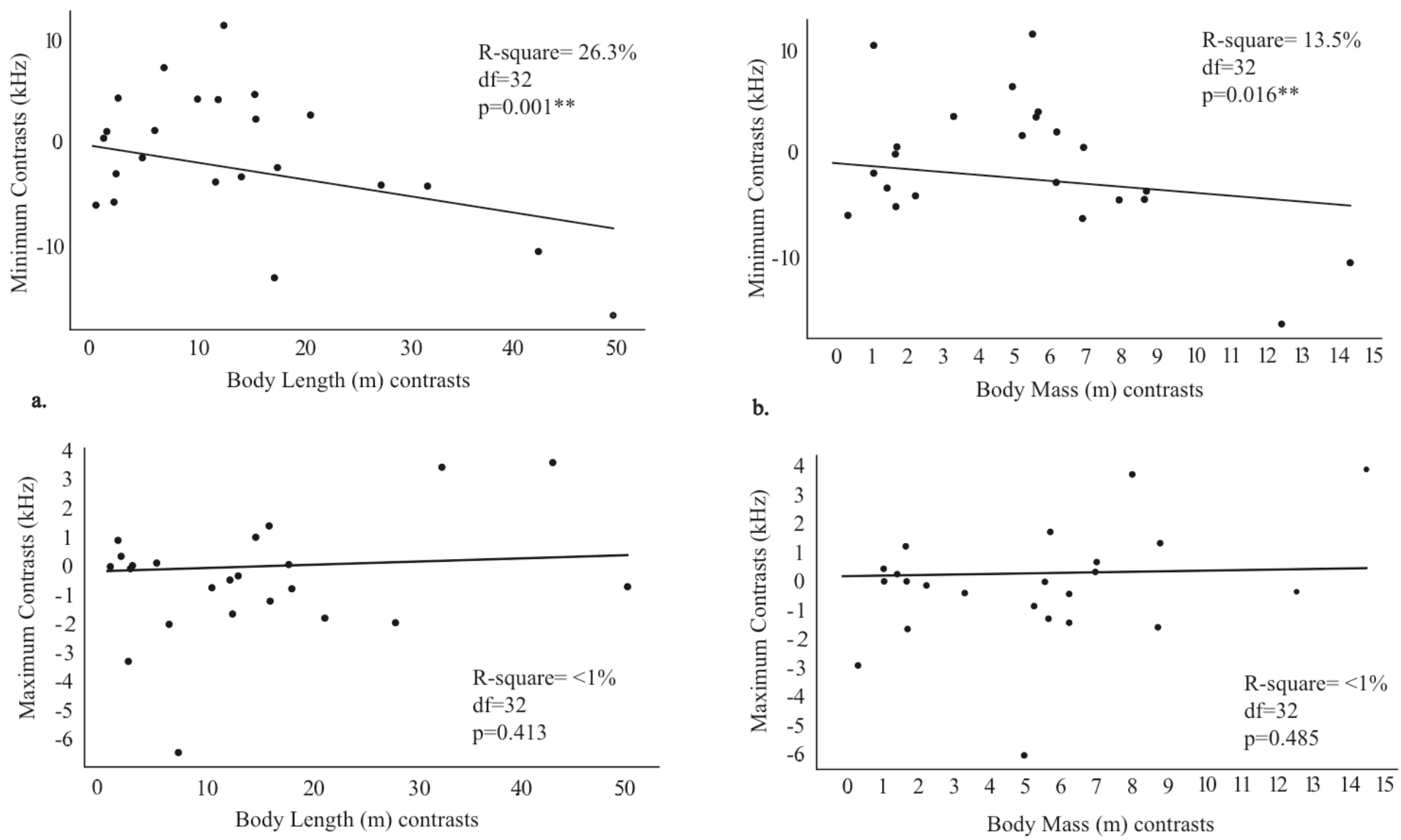

d. 
FIG.10

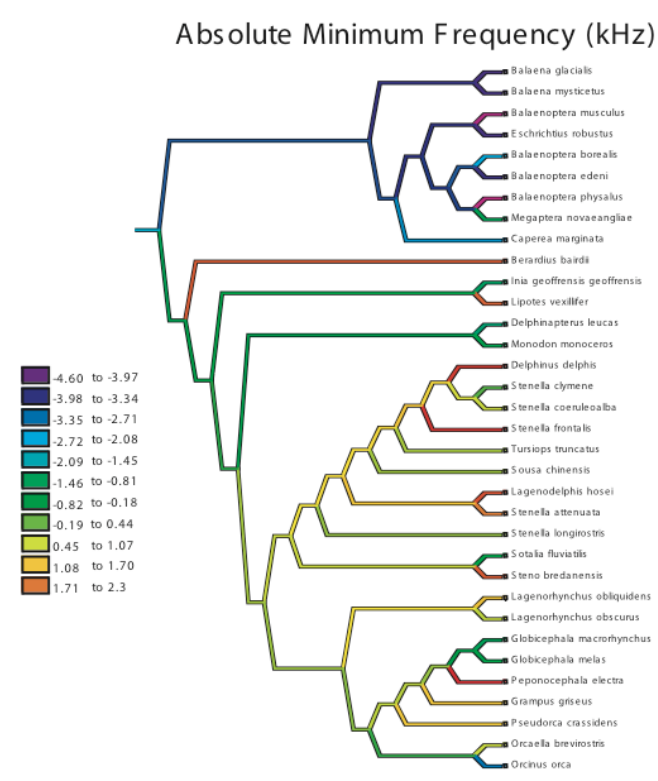

Absolute Maximum Frequency $(\mathrm{kHz})$

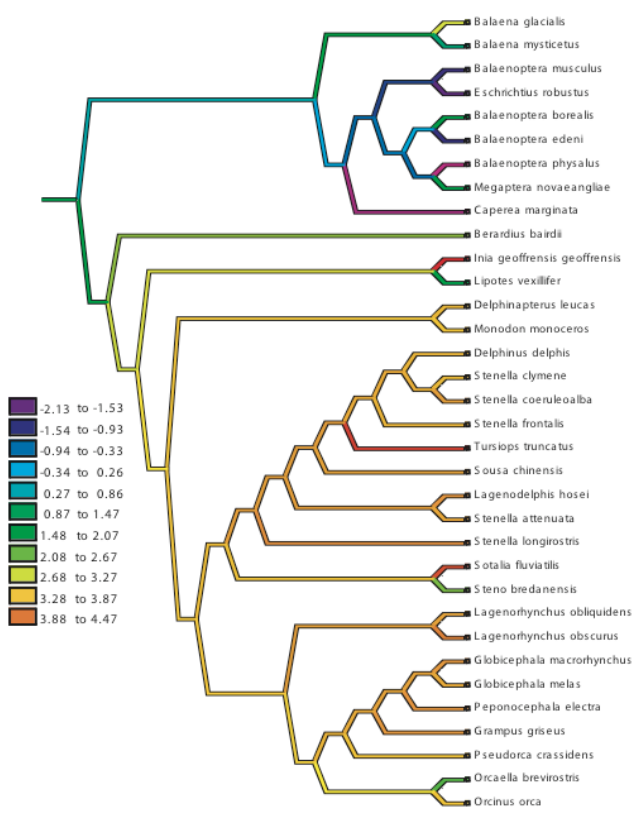

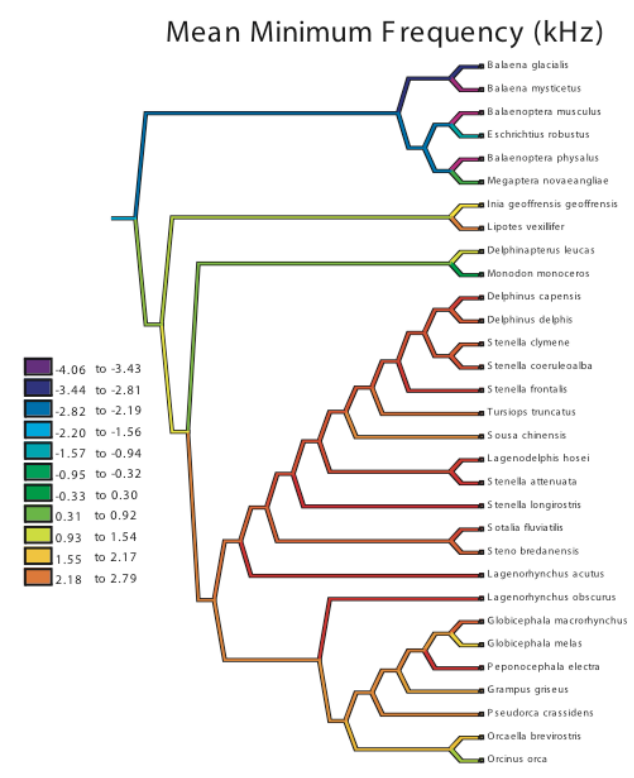

Mean Maximum Frequency $(\mathrm{kHz})$

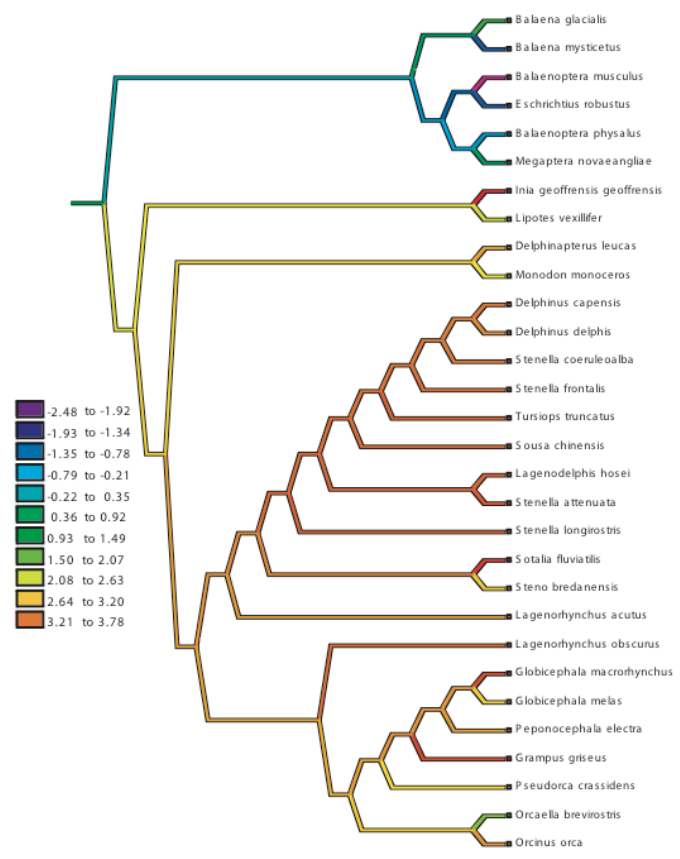




\title{
CHAPTER V
}

\section{PHYLOGENETIC REVIEW OF TONAL SOUND PRODUCTION IN WHALES IN RELATION TO SOCIALITY}

\author{
LAURA J. MAY-COLlado ${ }^{1,2}$, IngI AgnARsson ${ }^{3,4}$, DOUGlas WARTZOK $^{1}$
}

${ }^{1}$ Florida International University, Department of Biological Sciences, 11200 SW $8^{\text {th }}$ Street, Miami, FL 33199, USA

${ }^{2}$ Universidad de Costa Rica, Escuela de Biología, Apto. 2060 San Pedro, Costa Rica ${ }^{3}$ Department of Biology, University of Akron, Akron, OH 44325-3908, USA.

${ }^{4}$ Systematic Biology-Entomology, E-530, Smithsonian Institution, NHB-105, P.O. Box 37012, Washington, DC 20013-7012, USA

May-Collado, L. J., Agnarsson, I., and D. Wartzok. 2007. Phylogenetic review of tonal sound production in whales in relation to sociality. BMC Evolutionary Biology. 7(136). 


\begin{abstract}

\section{Background}

It is widely held that in toothed whales, high frequency tonal sounds called 'whistles' evolved in association with 'sociality' because in delphinids they are used in a social context. Recently, whistles were hypothesized to be an evolutionary innovation of social dolphins (the 'dolphin hypothesis'). However, both 'whistles' and 'sociality' are broad concepts each representing a conglomerate of characters. Many non-delphinids, whether solitary or social, produce tonal sounds that share most of the acoustic characteristics of delphinid whistles. Furthermore, hypotheses of character correlation are best tested in a phylogenetic context, which has hitherto not been done. Here we summarize data from over 300 studies on cetacean tonal sounds and social structure and phylogenetically test existing hypotheses on their co-evolution.
\end{abstract}

\title{
Results
}

Whistles are 'complex' tonal sounds of toothed whales that demark a more inclusive clade than the social dolphins. Whistles are also used by some riverine species that live in simple societies, and have been lost twice within the social delphinoids, all observations that are inconsistent with the dolphin hypothesis as stated. However, cetacean tonal sounds and sociality are intertwined: (1) increased tonal sound modulation significantly correlates with group size and social structure; (2) changes in tonal sound complexity are significantly concentrated on social branches. Also, duration and minimum frequency correlate as do group size and mean minimum frequency.

\section{Conclusions}


Studying the evolutionary correlation of broad concepts, rather than that of their component characters, is fraught with difficulty, while limits of available data restrict the detail in which component character correlations can be analyzed in this case. Our results support the hypothesis that sociality influences the evolution of tonal sound complexity. The level of social and whistle complexity are correlated, suggesting that complex tonal sounds play an important role in social communication. Minimum frequency is higher in species with large groups, and correlates negatively with duration, which may reflect the increased distances over which non-social species communicate. Our findings are generally stable across a range of alternative phylogenies. Our study points to key species where future studies would be particularly valuable for enriching our understanding of the interplay of acoustic communication and sociality. 


\section{BACKGROUND}

Cetacean tonal signals are broadly defined as narrowband, frequency modulated sounds [1-3]. Such sounds are produced by both baleen whales (Mysticeti) and toothed whales (Odontoceti) — sister clades containing all extant whales. They are also produced by other mammals [e.g., 4] and thus appear primitively present in the order. Baleen whales produce sounds that have fundamental frequencies generally below $5 \mathrm{kHz}[2,5]$, as do members of the sister lineage of Cetacea, the hippos [4]. In toothed whales, in contrast, these sounds most commonly range from $5-20 \mathrm{kHz}$ [2], and in some species, e.g. Delphinus delphis, Stenella attenuata, S. coeruleoalba, S. longirostris [6] Lagenorhynchus albirostris [7], Tursiops truncatus [8], fundamental frequencies can go as high as $48 \mathrm{kHz}$ in Inia geoffrensis [9]. In delphinid toothed whales these high frequency tonal sounds, especially when complex, are often referred to as 'whistles', although within the group whistle acoustic characteristics vary enormously. Several species produce both frequency modulated whistles (e.g., sine, convex, concave, upsweep, downsweep) and simple whistles that are relatively constant in frequency (e.g., Lagenorhynchus albirostris, [7]; Sotalia fluviatilis [10]; Stenella longirostris [11], others are limited to simple whistles (Lipotes vexillifer) [12] or to few frequency modulated whistles (e.g., mostly downsweep in Inia geoffrensis) [9]. In addition, whistle contour may be continuous or consist of a series of breaks and segments [2]. Whistles may or not contain harmonics [2]. In delphinid species like S. longirostris [13] and L. albirostris [14] whistles can contain high order-harmonics. Finally, whistle duration is very variable. For instance, in Sousa chinensis whistles can range from 0.01 to 1.3 seconds [15] and in Tursiops truncatus from 0.05 to 3.2 seconds [16]. In delphinids, whistle frequency 
modulation and duration varies within species in relation to geography [e.g., 10, $11,16,17]$, and related species differ in many whistle frequency components (e.g., maximum, minimum, end, and start frequency) [e.g., 18-22].

Baleen whales produce a great variety of sounds, among them tonal sounds that like toothed whale 'whistles', are narrowband and frequency modulated, although typically much lower in frequency [1]. These tonal sounds can be produced in isolation or in combination with other sounds (e.g. pulsative sounds). In the Right whale (Balaena glacialis) these tonal sounds, again like 'whistles' in toothed whales, are used in a social context [23]. For example, in Blue whales (Balaenoptera musculus) tonal sounds are presumably used for long-distance communication [24], and in Right whales tonal sounds are used in combination with pulsative sounds in a sexual context [25]. However, in baleen whales, these tonal sounds are never referred to as whistles, but as 'calls', 'moans' or 'tones' [26, 24, 27-29]. Nomenclature of sounds, both in toothed and baleen whales, is confusing. As stated by Au (2000: 31) [1] in baleen whales "as with dolphins there is a lack of any standard nomenclature for describing emitted sounds", this frustrates comparison of sounds across taxa and obscures homologies. It remains unclear exactly what is a 'whistle', and if narrowband, frequency modulated tonal sounds of baleen whales and toothed whales are homologous at some level. One reason to question tonal sound homology across whales is that the sound production mechanisms of baleen whales and toothed whales are dramatically different. In baleen whales tonal sounds are thought to be laryngeal $[30,31]$, as they are in other related mammals [e.g. 4, 32], but in toothed whales sounds are produced by a unique and complex nasal system [e.g. 33, 34]. This offers some support for the hypothesis that toothed whales 'whistles' are unique and 
different from (not homologous with) baleen whale tonal sounds. However, this also suggests that high frequency tonal sounds are homologous across toothed whales and such sounds in non-delphinid toothed whales should also be called whistles (contra Podos et al. 2002) [35]. To accommodate both possibilities we do all analyses across all whales (allowing for potential homology of tonal sounds across the order) and separately within toothed whales.

Most of the work on whistles has been done with social delphinids, where they are often referred to as "social signals" and are thought to facilitate individual recognition, group cohesion, recruitment during feeding activities, and overall communication [e.g., 1, 3, 36-44]. Generalizations about the function of whistles have translated into the hypothesis that whistles evolved in concert with sociality, and that the two traits are tightly correlated [e.g., 45, 35]. Herman and Tavolga (1980) [45] suggested that the degree of gregariousness in toothed whales seemed to be related to whistle production [see also 46]. More specifically, they proposed that species that live in small groups or are solitary tend not to whistle, whereas species that live in large groups frequently do. Recently, Podos et al. (2002) [35] proposed that whistles are an innovation of social delphinids; in other words that whistles are synapomorphic for a clade within Delphinidae. However, even within delphinids some social species such as Cephalorhynchus spp and some species of Lagenorhynchus do not whistle [e.g., 46, 47], which seems to contradict the dolphin hypothesis. The hypothesis was furthermore based on an assumption of the absence of whistles in river dolphins (Inia, Lipotes, Platanista, and Pontoporia), porpoises (Phocoenidae), beaked whales (ziphids) and belugas and narwhals (Monodontidae). However, we do not believe this assumption is justified. Tonal 
sounds from Inia geoffrensis, for example, have been independently recorded in several studies $[9,21,22,48]$. These sounds, just like in other toothed whales, have been referred to as whistles, although they are simpler and shorter in duration, and higher in frequency than the whistles of some dolphins [9]. Similar whistles have also been reported in another river dolphin Lipotes vexillifer [e.g., 12, 49, 50] and in social non-delphinid toothed whales such as some beaked whales [51, 52], and the Monodontidae, belugas and narwhals [e.g. 53-57). Podos et al. (2002) [35] concluded that the tonal sounds in these species should not be classified as 'whistles', and hence found support for the dolphin hypothesis. While we agree with Podos et al. that whistle structure seems different in delphinids and non-delphinid toothed whales we believe this demonstrates the basic problem of treating broad, arbitrary, concepts as single traits in evolutionary analyses. To define whistles as social sounds produced by delphinids - a priori denying homology with tonal sounds in related taxa - and then concluding that they evolved in association with sociality in Delphinidae risks circularity. In such a framework reconstructing the origin of 'whistles' on a phylogeny will simply depend on the whistle definition chosen by any given author.

To facilitate discussion, and comparability with previous research, we use the word 'whistle' for toothed whales tonal sounds, however, we do not imply that whistles are necessarily non-homologous to baleen whale tonal sounds - their homology requires further study. We use whistles as a category for some of our analyses, mainly to test the dolphin hypothesis as it was proposed. It is not very informative, however, to simply map the distribution of 'whistles' on a phylogeny (Fig. 11, [see Appendix 1]). Authors differ in their interpretation on the presence or absence of whistles across species, e.g. some define 
them in the context of a behavior that may have much more limited distribution than the sounds themselves. Furthermore, even within dolphins 'whistles' can be highly variable. We thus highlight the need to focus on the various acoustic parameters (such as frequency variables, modulation, etc.) that may vary independently and have nonidentical phylogenetic distributions [see Appendix 1 for rationale]. Hence, our major focus is on such analyses which may reveal which, if any, of the characteristics of 'whistles', or tonal sounds in general, seem associated with sociality.

Our understanding of tonal sound acoustic structure, diversity, and use, is growing, but the evolution of tonal sounds and their association with sociality remains highly speculative. We therefore believe we here improve upon previous studies by providing a more detailed analysis, and using novel and more detailed phylogenies than any study hitherto. We also test these hypotheses across a range of alternative phylogenies.

In sum, we here review current knowledge of both tonal sound production and social structure in Cetacea, and explore the evolution of tonal sounds and the association of individual tonal sound components with sociality (overall social structure and social components). Taking advantage of a new species-level cetacean phylogeny $[58,59]$ we provide the first phylogenetic test of the hypotheses of Herman and Tavolga (1980) [45] and Podos et al. (2002) [35]. This study identifies large gaps in knowledge on both traits, and points to key species where future studies would be particularly valuable for enhancing our understanding of the interplay of tonal sounds and sociality. 


\section{RESULTS}

\section{Testing the Dolphin Hypothesis}

The following is presented merely to test the dolphin hypothesis as stated (see Introduction, Methods, and [see Appendix 1] for problems with this coarse approach). Under the definition of 'whistle' we use here, the optimization of whistles on the phylogeny is ambiguous (Fig. 11b). However, all of the equally most parsimonious reconstructions reject the dolphin hypothesis. The phylogeny implies that whistles either evolved independently twice, once in Berardius and once in the node leading to Delphinida sensu Muizon (1988) [60], delphinoids plus river dolphins + Platanista (a clade we here refer to as Pandelphinida), with secondary losses in Phocoenidae and within Delphinidae (Cephalorhynchus spp. and Lissodelphis spp.). Alternatively whistles evolved once in the common ancestor of ziphiids plus pandelphinids and then were subsequently lost thrice in Hyperoodon, phocenids and within delphinids (the optimization of whistles is equally ambiguous on previously published phylogenies, [see Appendixs 2-4], while dual origin of whistles is better supported when optimized across the entire set of filtered post-burnin trees, see Methods). Likewise, there are two possible optimizations of sociality under a broad concept approach. One is that sociality evolved in the common ancestor of Odontoceti and was then lost secondarily twice in the riverine species (Fig. 11b). Alternatively sociality may have evolved independently four times (in Physeter macrocephalus, within Ziphiidae, Pontoporia, and in Delphinoidea). The optimization of sociality is ambiguous on over $99 \%$ of the alternative trees examined, however, the multiple loss of sociality within Cetacea seems more likely in general, given 
that relatives of whales are social. Regardless of choice of optimizations, whistles did not originate in the lineage leading to the social dolphins, contra the dolphin hypothesis.

\section{Character Optimizations}

Results of character optimizations led to the same conclusions across all alternative phylogenies examined (previously published hypotheses, [see Appendixs 2-4], and postburnin trees from our Bayesian analysis of Cytochrome b), unless otherwise noted.

Group sizes in Cetacea [see Appendix 5] appear to have been ancestrally small, but to have gradually increased in the lineage leading to the dolphins, with a number of independent derivations of societies with hundreds of individuals and some secondary reductions in group size (e.g., Cephalorhynchus spp, Orcaella and Orcinus Fig. 12).

Here we present some alternative optimizations of sociality under both a 'broad two and four state concept' framework simply to test the dolphin hypothesis and under a multiple component framework. We note, however, that our study offers limited insights into the evolution of sociality in cetaceans. Future studies will require examining a greater number of component characters of sociality as such data becomes available, and it will require the inclusion of comparative social data also from the outgroups.

We compare three optimizations of sociality represented as a four-state character (social structure) (see Table 8 and [see Appendix 5]). First, we keep polymorphic species (species reported to show more than one type of social organizations) as such and then compare results when the 'lowest' and 'highest' social state is chosen for each polymorphic species (Fig. 13). All three optimizations have some ambiguity, but optimizations across all trees suggest that family based groups evolved independently at 
least three times (Physeter, Monodon, and Globicephalinae Fig. 13,). The optimization of social components (including polymorphism) is shown in additional material [see Appendix 6]. Group composition appears to have ancestrally been simple groups consisting only of mother and calf. Segregated (by sex and/or age) and mixed groups may have evolved independently at least four times [see Appendix 6b]. Finally, member associations appear to have evolved from simple mother and calf interactions to complex family based associations [see Appendix 6c].

Figure 4 shows the optimization of each acoustic character (all transformed using the natural $\log$ ). Relatively high maximum and minimum frequencies (both absolute and mean) appear derived in toothed whales (Fig. 14a-b,d-e). Particularly high mean maximum and minimum frequencies have evolved within delphinids (note that some of the variation within delphinids and other groups is visually masked by the way Mesquite groups continuous variables in color ranges; [see Appendix 7 for greater detail].

There appears to be a similar trend in the number of tonal sound inflection points (an indicator of tonal sound complexity) going from few ancestrally and increasing in the lineage leading to the dolphins (Fig. 14f). There is an inverse trend in tonal sound duration, where particularly short tonal sounds appear to be derived within the delphinids (Fig. 14c).

\section{Character regressions and correlations}

Under the independent contrast method the regression between group size and the mean number of inflection points was marginally significant: species with larger groups tend to produce tonal sounds with greater mean number of inflection points. Group size 
explained approximately $7.9 \%$ of the variation in inflection points across cetaceans ( $p=0.05, \mathrm{df}=33$ see Fig. 15 (this and some of the following results are dependent on the choice of phylogeny, see section Phylogenetic uncertainty). Group size also significantly explained variation in the mean minimum tonal sound frequency within toothed whales $\left(\mathrm{R}^{2}=12.4 \%, \mathrm{df}=23, \mathrm{p}-{ }_{1 \text { tailed }}=0.04\right)$. We justify using a one-tailed test based on the expectancy that low frequency sounds travel longer distances so that a priori one might expect that low frequency tended to be associated with solitary species, while species that live their entire lives in large groups need only communicate over short distances. However, given that the two tail test is non-significant we consider this hypothesis only weakly supported. Regressions between group size and other acoustic parameters were not significant.

In addition, there is a significant negative relationship between tonal sound duration and absolute and mean minimum frequency both for all cetaceans (Abs-MinF, R-square $=17 \%, \mathrm{p}=0.02, \mathrm{df}=31$, Mean-MinF, $17.5 \%, \mathrm{p}=0.02, \mathrm{df}=29)$ and for toothed whales $($ Asb-MinF, R-square $=38 \%, p<0.001, d f=22$, Mean-MinF, R-square $=24 \%$, $\mathrm{p}=0.01, \mathrm{df}=23$ ). There was a significant positive relationship between tonal sound duration and complexity for all cetaceans $(\mathrm{R}$-square $=12 \%, \mathrm{p}=0.04, \mathrm{df}=32)$ and for toothed whales $(\mathrm{R}$-square $=45 \%, \mathrm{p}<0.001, \mathrm{df}=23)$.

Changes in tonal sound complexity were significantly concentrated within social lineages in four of the five most parsimonious reconstructions when both traits were treated as two state characters [see Appendix 8] f.

Tests of character state associations (SIMMAP) show that complex whistles (state $1=$ more than one inflection points) were positively associated with group living species 
$(\mathrm{Dij}=0.13, \mathrm{p}>0.999)$ and negatively with less social species $(\mathrm{Dij}=-0.024, \mathrm{p}<0.001)$ treating social complexity as a two state character. In general there was an association between tonal sound complexity and social structure $\left(D_{\text {statistic }}=0.376, p<0.001\right.$, Table 9$)$. However, the associations between individual states vary depending on how finely tonal sound and social characters are divided (Table 9). For instance, when treating social complexity as a four state character but tonal complexity as a two state character we find a significant positive association between highly social species (states 2 and 3 ) and complex tonal sounds and a negative association between complex tonal sounds and 'solitary' (state 0 ) species (Table 9). When both are treated as four state characters only negative associations are significant (but in the same directions as before, see Table 9).

When three component characters of sociality were analyzed we found similar significant character associations with inflection points (Group size $\mathrm{D}_{\text {statistic }}=0.394$, $\mathrm{p}<0.001$; Group Composition $\mathrm{D}_{\text {statistic }}=0.306, \mathrm{p}<0.001$; Stability/Associations $\mathrm{D}_{\text {statistic }}=0.364, \mathrm{p}<0.001$, [see Appendix 9 and legend for detail], all indicating association between complex whistles and high levels of sociality.

\section{Phylogenetic uncertainty}

In general, most of our findings are not strongly dependent on the phylogeny of choice, as long as all the species are included. In other words, results in most cases are similar whether the data are analyzed across the trees favored by our own analyses (all post burnin trees and post burnin trees filtered using agreement among multiple studies), or restricted to trees filtered to be congruent with the alternative hypotheses of Messenger and McGuire (1998) [61], Nikaido et al. (2001) [62] or Arnason et al. (2004) [63], 
respectively (see Methods for detail). On the all-species phylogenies results significant in the main analyses were also significant across all sets of trees for all SIMMAP analyses. The only difference between analyses was that social and whistle character states were more strongly associated on the trees constrained by the Messenger and McGuire hypothesis than in the remainder [see Appendix 10]. Similarly, the PDAP analyses results agree irrespective of phylogeny choice [see Appendixs 11 and 12], except the following. Group size and number of inflexion points correlate significantly except on trees constrained by the hypotheses of Arnason et al. (2004) [63] or Nikaido et al. (2001) [62], and group size and mean minimum frequency correlate except on trees constrained by the Messenger and McGuire (1998) [61] hypothesis. For ancestral character reconstruction under parsimony, the optimizations of the continuous characters such as group size, tonal sound frequencies, duration, and inflexion points are nearly identical across the trees considered. The optimization of whistles as a presence/absence character was ambiguous on our, and previous, phylogenetic hypotheses. However, on $70 \%$ of the filtered post-burnin trees dual origin of whistles was preferred (see above). The optimization of sociality (as a two state character) was ambiguous (single origin followed by multiple losses, or two origins followed by fewer losses), except on the Nikaido et al. (2001) [62] hypothesis which favors two origins of sociality. Similarly optimizations of whistles and sociality as multistate characters varied little across trees with no impact on conclusions.

When we used the phylogenies resulting from reanalyzes of the data of Messenger and McGuire (1998) [61], however, significance was lost in a higher number (although not the majority) of the hypotheses tests [see Appendixs 10-12] and some character 
optimizations changed. Although this can in theory imply sensitivity to phylogenetic pattern, a simpler explanation for this finding seems to be that much of the power of the comparative tests is lost as Messenger and McGuire's data [61] includes only a portion of the species of our main dataset. Hence we do not see a reason to discuss these 'disagreements' further. 


\section{DISCUSSION AND CONCLUSIONS}

Our results show that the interplay of tonal sounds and sociality is complicated and that studying the relationship between conglomerate characters such as 'whistles' and 'sociality' largely conceals these intricacies. Under the very simple 'concept approach' the cladistic test [see 64] rejects the dolphin hypothesis stating that 'whistles' evolved as an adaptation for social communication in dolphins. Whistles, as here defined, appear to be a synapomorphy of pandelphinids, or even a more inclusive group including ziphiids (Fig. 11b). Therefore, the current evidence implies that whistles arose earlier in the evolutionary history of whales than presumed by Podos et al. (2002) [35], and whistles are furthermore present in some non-social species, and have been lost more than once within social clades. Apparently then, whistles are not necessary for functional cetacean societies and social communication, and they can play some role in communication in solitary species.

Our findings highlight some of the problems with evolutionary analyses of imprecise, broad concepts. Even though 'whistles' do not correlate with any measure of sociality we find evidence that the evolutionary histories of sociality and tonal sounds are intertwined in the direction suggested by many authors, including Podos et al. (2002) [35]. This is evidenced mainly by two findings. (1) The significant association between group size and tonal sound inflection points (complexity) whether tested using independent contrasts, concentrated changes, or character association tests; and (2) the association between group size and minimum tonal sound frequency (and the association of the latter with duration). Simple tonal sounds are mostly confined to species with simple societies (mostly solitary) such as river dolphins and rorquals while tonal sound 
and social complexity increase in the lineage leading to Delphinoidea (Tables 2). Within that lineage reversal to simpler societies has occurred twice and each time tonal sounds have been secondarily lost (Figs 1a-b, 3), although whistle loss may represent a response to predatory pressure rather than change in social structure (see below).

In addition, especially in toothed whales, species emitting longer tonal sounds tend to show a greater number of inflection points. These observations and tests are congruent with hypotheses stating that complex tonal sounds function as social signals for group cohesion (e.g., most delphinids) during social, traveling, and feeding activities [e.g.,42, 65] or individual recognition (e.g., bottlenose dolphins, Atlantic spotted dolphins) [e.g., 3, 37, 41, 66, 67].

But functionality in a social context can only explain a portion of the variation in tonal sound production and complexity. The secondary loss of tonal sounds in porpoises and the dolphin clade containing Lagenorhynchus australis, L. cruciger and Cephalorhynchus spp, for example, suggests these signals may sometimes be costly, for example in terms of energy production or predation risk. These odontocetes live in very fluid societies where acoustic communication is accomplished by means of rapid pulsed sounds $[47,68]$. One potential costs of tonal sounds is that these signals may be intercepted (eavesdrop) by an unintended receiver [69, 70]. Delphinid tonal sounds are within a frequency range that is readily detected by predators like killer whales which are known to predate on many marine mammal species including these non-whistling species. Furthermore, porpoises and Cephalorhynchus seem to have converged upon similar morphology and biosonar systems [71, 72], both have ears tuned for high frequency sounds and produce narrowband clicks [73] that are used for echolocation 
purposes and communication [74, 75]. As emphasized by Morisaka and Connor (2007) [76] if killer whales poorly detect these signals, then it may be beneficial for these species to use high frequency signals for social communication $[73,74]$ instead of tonal sounds.

In stable societies like those of Physeter macrocephalus and Orcinus orca, animals tend to produce group-specific sounds (termed codas and calls respectively) whereas in fission-fusion societies like those of Tursiops truncatus and Stenella frontalis, animals produce individual-specific whistles, so called "signature whistles" [see 3,15 , 41, 38]. Signature whistles are sounds (single-loop and multiple-loop) [see 75] that to date have only been found in species with fluid societies where mother and calf use them as contact calls and some animals (particularly males) form coalitions (individual recognition may be important when forming these alliances) [e.g., 15, 37, 38, 44, 66, 67, $73,77-82]$.

We found evidence for association between group size and the mean minimum frequency, as well as between mean minimum frequency and duration. Given that the former was only marginally significant, we will not place much emphasis on this finding. However, if this finding will be better supported with the addition of further data it may suggest that low minimum frequency (and long duration) is selected for in mostly solitary species which must communicate with other individuals over relatively greater distances than do species that live in permanent societies. It should be noted that May-Collado et al. (2007) [59] found a correlation between minimum frequency and body size across whales. This may explain a part of the observed pattern here, as social species are often small, but it remains to be explored if sociality and body size are correlated. 
Despite the possible differences in the context in which tonal sounds are produced by riverine dolphins and other delphinoids, there is no a priori reason to assume that whistles produced by these toothed whales are not homologous (contra Podos et al. 2002) [35], and phylogenetically their homology is supported (Fig. 11). It has been proposed that marked deviations of Inia from delphinids in scaling relationship in body size and frequency [e.g., 21, 83] is evidence that their sounds are produced by mechanisms different from those used by delphinoids. This is primarily based on the assumption that vertebrate scaling of vocal frequency occurs through size-dependent effects on a common vocal apparatus [e.g. 80], thus deviations from scaling relationships might indicate an independent proximate mechanism [35]. However, these scaling patterns, for maximum frequency disappear once phylogenetic relationships are taken into account [59].

While some cetacean societies have been studied for a long time, detailed observations are lacking for many species and it is difficult to define and compare levels of sociality across cetacean species. Likewise there are many gaps in our knowledge of tonal sound production [see Appendixs 5 and 7]. Our study highlights critical gaps in knowledge, and pinpoints key taxa whose future study could quickly enhance our understanding of the evolution of tonal sounds. As can be seen in Figure 1, tonal sound data would be especially valuable from Kogia, ziphiids other than Berardius, and from Platanista and Pontoporia. In a similar manner information on social structure of Kogia, Mesoplodon, and Ziphius would help resolve the optimization of sociality.

Many factors in addition to sociality have been proposed to have influenced the evolution of tonal sounds, including body size and maximum frequency scaling [21, 35, $59,83,84]$, habitat [21], predation [76], and zoogeographical [20] and phylogenetic 
relationships $[20,21]$. Given that multiple factors are at work true co-evolutionary histories of any given characters could easily be masked. Hence, finding significant correlations between tonal sounds and social structure is particularly interesting. For example, we find a significant, but rather weak, correlation between group size and inflexion points using the independent contrast method. One of the conspicuous outliers in this analysis is Orcinus orca, a social delphinid living in relatively small groups that nevertheless produces extremely modulated whistles. Thomsen et al. (2001) [85] discuss these extreme modulations and suggest that whistles in killer whales serve a different function than in related dolphins. Removing $O$. orca from the analyses increases the strength of the correlation between whistle complexity and group size (R-square $=9.7 \%$, $\mathrm{p}$-value $=0.03$ ). It should furthermore be noted that comparative biology is fraught with difficulty, getting enough data together for a strong hypothesis testing is typically difficult and missing data results in a loss of power. By accounting for uncertainty in phylogenetic relationships we hope to reduce the rate of type I error. Further, accounting for differences in interpreting and scoring whistle and sociality data attempts to reduce type I error. It is quite possible that in an attempt to avoid type I error we are introducing an unacceptable amount of type II errors. In other words, our ability to detect true character correlations in evolutionary history may be compromised. In this study, however, most of the results were not sensitive to choice of phylogeny or alternative scoring scenarios which adds some confidence to our conclusions.

Our findings point to gaps in knowledge of both tonal sounds and social structure that need to be filled to significantly advance our understanding of their putative coevolutionary histories. Nevertheless, our results allow us to reject the simple hypothesis 
that 'whistles' evolved for social communication in dolphins. However, group size explains some of the variation in tonal sound frequency and frequency modulation indicating a special role for complex tonal sounds in a (complex) social context and perhaps for low frequency, long-duration sounds in solitary species. May-Collado and Wartzok (2007) [9] suggested that whistles in Inia geoffrensis may be use to keep distance between animals rather than to stimulate social interactions. However, this hypothesis needs to be tested. Future studies should focus on particularly poorly known groups of species such as riverine species, ziphiids, and Kogia spp. 


\section{METHODS}

\section{Definitions}

For purposes of this study the association between tonal sounds and sociality will be studied under both a broad concept [tonal sounds and whistles versus sociality, emulating previous studies], and using a 'component' approach whereby tonal sounds and sociality are dissected into (some of) their component characters. For tonal sounds, standard acoustic parameters we use here include absolute and mean minimum and maximum frequencies $(\mathrm{kHz})$, duration $(\mathrm{s})$, and number of inflection points (a measure of whistle modulation, and a proxy for whistle complexity) [see Appendix 7].

Current knowledge on cetacean sociality indicates the existence of a wide range of social structures, ranging from 'solitary' to highly structured group living species [see 86]. Generally in the study of cetacean sociality, social species are those that show evidence of group living [87] where animals are associated in a nonrandom fashion [88]. Under the broad concept approach, we have classified species into two general social frameworks, one simply organizing species into non-group living species (state 0) and group living species (state 1) and a second one assigning species to four social types (Table 8, [see Appendix 5]. Under the component approach, we also examine some component characters of sociality for which there is sufficient data available (group size, composition, and stability/associations) either from short and/or long term studies as well as anecdotal observations (Table 8, [see Appendix 5]). Table 8 provides detailed descriptions of these character and their states. It is important to note that for any type of qualitative characterization of sociality, some species may fit into more than one category due to intraspecific variation. For instance, some populations of Stenella longirostris 
have unstable (or 'fluid') groups whose compositions change throughout the day, while populations in the Hawaiian atolls exhibit long-term group fidelity and social stability [89]. These, and other limitations of this study should be kept in mind when interpreting our findings, nevertheless, we believe our approach improves upon previous attempts to detect the associations between sociality and tonal sound production in whales.

\section{Character Optimizations}

Published data on cetacean tonal sound production and sociality were obtained from literature and personal communications [see Appendixs 5 and 7]. For tonal sounds we compiled information on the most used acoustic parameters: absolute and mean minimum frequency, absolute and mean maximum frequency, duration, and mean number of inflection points. We only considered studies conducted in the wild or in captivity where, based on the information provided by the authors, it could be assumed species were not recorded in mixed-species groups. We assumed authors were not including harmonics in the acoustic measurements of the tonal sounds emitted by the studied species, unless specified. Information about the social structure of cetaceans was obtained from short to long-term studies, as well as anecdotal information. We searched for information for each of the following social components group size, composition, stability and associations patterns. In addition, information on these social components was used to define four social categories. A minimum of two components was required to place a species within a social category as defined in Table 8 . Species for which insufficient components were available were coded as unknown. For species with populations that varied in their social structure or any of the social components ('polymorphic') we selected the highest social 
state for that particular character. Group size is analyzed as a continuous character using the highest mean group size found in the literature, and also as a discrete character which allows the inclusion of more species [see Appendix 6] since many authors do not provide a mean value but instead offer a description of group sizes.

We relied upon the recent species level phylogenies provide by May-Collado and Agnarsson (2006) [58] and May-Collado et al. (2007) [59]. All the main analyses were made using the preferred tree from May-Collado et al. (2007) [59] [see Appendix 13]. Because polytomies can compromise character optimization and tests of character correlations, characters were optimized on a fully resolved tree, which is the majority rule tree resulting from a MrBayes analysis (see May-Collado and Agnarsson 2007 for details) [58] without collapsing nodes with less than 50\% frequency (using the contype $=$ allcompat option). However analyses were also run on a range of alternative phylogenies (see below) Character optimization was performed with the program Mesquite 1.12 [90], using weighted squared-change parsimony [91].

Acoustic characters were optimized in two data sets (1) with of all cetacean species and (2) pruning species that are known not to emit tonal sounds, species for which acoustic behavior is poorly known, and species that are known to produce tonal sounds but for which detailed information for the character under study was not available. When several values were reported in a species for a particular trait the largest maximum frequency and duration, and the smallest for minimum frequency were used for the analyses [see values in bold in Appendix 7]. Number of inflection points was analyzed both as continuous, reflecting the continuous nature of the data, but also as a two and four 
state discrete character to facilitate additional analyses that require ordinal data (Table 8 , [see Appendixs 5 and 9]).

Sociality was optimized as discrete two and four state characters, and using the social components: group size, composition, stability and association patterns (Table 8, [see Appendix 5]). Because several species were polymorphic for one or several characters we optimized species in three ways (1) as polymorphic, (2) emphasizing their 'highest' social level reported, and (3) emphasizing their 'lowest' social level reported. Finally, we analyzed group size as a continuous character.

\section{Independent Contrasts}

Assuming group size as a coarse proxy for social complexity (as defined above by Connor 2000) [87] we regressed it against tonal sound parameters to examine the association of sociality and tonal sound production. Contrasts were calculated using the method of phylogenetically independent contrasts [92]. The method takes into account known dependencies among observations due to phylogenetic relationship of species, and therefore reduces error [93]. Independent contrasts were calculated using the PDAP: PDTREE module [94, using an unpublished version provided by P. Midford] in Mesquite 1.12 (build h47, 85). To estimate independent contrasts, branch lengths were used as estimated by MrBayes; branch length transformations were necessary for group size (Lack of fit test $\mathrm{p}<0.05$ ) and were exponentially transformed. We also tested the relationship between tonal sound frequency and complexity [mean number of inflection points] and tonal sound duration using the independent contrast method. 


\section{Character correlations}

We also tested character associations between discrete characters of sociality and tonal sound complexity using two different methods. First we used the software SIMMAP 1.0 [95] which allows for multistate character associations. We did the following tests using all post-burnin trees ( $\mathrm{n}=2000)$ from our Bayesian analysis (May-Collado et al. 2007) [59] using default settings of the program and employing a rough false discovery rate (FDR) to correct for multiple simultaneous comparisons (critical p values for tests of 8,12 , and 16 comparisons are $0.028(0.972), 0.027$ (0.973), and 0.27 (0.973), respectively). We tested the association of (1) sociality and tonal sound complexity both scored as two state characters, (2) social structure and tonal complexity scored as four state characters, and (3) each of the social components and tonal sound complexity scored as two and four states characters [see Appendix 5]. Second using the concentrated changes test [96] in the software MacClade [97] we tested if changes in tonal sound complexity were concentrated on social branches. For this test we used only two state characters.

It is important to note that testing the role (if any) of sociality in tonal sound evolution is challenging due to the large gaps in our knowledge of cetacean societies, difficulties of objectively defining tonal sound complexity, and levels of sociality, and the limitations of available methods. We note that, as with all of the ordinal data we use here, the divisions between character states are rather arbitrary and open to criticism and alternative coding. Nevertheless we believe that our, be it coarse, phylogenetic approach represents an advance over previous studies that have speculated on social and whistle evolution using less data and lacking a phylogenetic reference. We have tried to test the association of characteristics such as group size and whistle parameters using various 
different approaches (independent contrast test, concentrated changes test, pairwise comparisons on the phylogeny, and character association test for multistate characters), testing them across various alternative phylogenies, and our results are presented in the form of hypotheses that we hope will subsequently be better tested upon the availability of more data and more sophisticated methods. Also, importantly, our data highlight gaps in knowledge and should guide future studies to where allocating resources might be most beneficial.

\section{Current Knowledge on Cetacean Sociality and Tonal Sounds}

Connor et al. 1998 [86] and Matthews et al. 1999 [83] provided brief reviews of the evolution of sociality in toothed whales and tonal sounds in cetaceans, respectively. Connor et al. 1998 [86] review highlighted the lack of knowledge for most toothed whale species and focused on the social structure of a few species including Tursiops truncatus, Orcinus orca, Globicephala spp., Berardius bairdii, Physeter macrocephalus. They compared toothed whale social structure with some terrestrial mammals e.g. elephants and chimpanzees, and found both similarities between the two, but also identified some social elements unique to toothed whales. Matthews et al. 1999 [83] summarized the frequency and time parameters of 40 cetacean species tonal sounds in relation their body size.

This review summarizes information from 335 sources on sociality and tonal sounds for 64 and 36 Cetacean species, respectively [see Appendixs 5 and 7]. The information was gathered from via searches on Web of Science and Google Scholar, and 
include scientific papers in peer-reviewed journals, conference abstracts, M.Sc. theses, Ph.D. dissertations, technical reports to international organizations, etc.

Although not the main aim of this paper, a few summary statements can be made about current knowledge of sociality and tonal sound production in whales [see Appendixs 5 and 7]. Baleen whales have a rather uniform social structure, generally live in simple societies where animals spend considerable time solitary. Weak associations are limited to aggregations form during the breeding and feeding time, and long-term associations appear to be limited to the time mother and calf remained together. In contrast, toothed whale social structure varies enormously, ranging from solitary to species living in huge groups. In groups, group members show an array of association patterns, from weak to stable family associations. For porpoises (Phocoenidae) and several of the freshwater cetacean species (e.g., Platanista, Lipotes, Inia) authors have described group member associations as 'undeveloped', 'weak', or 'fluid'. Such description are difficult to interpret and do not necessarily mean that the authors are suggesting these species live in a fission-fusion society as reviewed in Connor et al. 1998 [86] for Tursiops truncatus. For most delphinids, association patterns have been described as 'fluid', 'highly fluid fussion-fusion', or 'fluid with short-lasting associations'. In these cases authors appear to imply by 'fluid' that the species do live in fission-fusion societies [as described by 86]. In these species males tend to form coalitions and alliances to 'capture' and maintain consortship with females. Finally, the most stable social structures have been described in the Sperm whale, (Physeteroidea), most members of the subfamily Globicephalinae, and possibly the Narwhal (Monodontidae). Notably, these species are not all closely related so that "stable" 
societies have evolved convergently, however, species differ in the degree of dispersal particularly male dispersal from the group.

Our review updates Matthews et al. (1999) [79] review on Cetacean tonal sounds. We included recently reported information on species like Delphinus capensis and Sotalia guianensis [see Appendix 7]. We also updated information on several others like the Narwhal and Beluga (Monodontidae) and the river dolphins Lipotes and Inia where more data has become available. The previous review [83] included tonal sound information from two beaked whale species (Mesoplodon densirostris, M. carlhubbsi) that we considered controversial due to the possible pulsative nature of these sounds, thus exclude this information from the table. In addition, Sousa chinensis and Sousa plumbea were considered here a single species, since no clear evidence yet exists to separate them into two distinct species. Likewise, we consider Stenella plagiodon as a synonym of Stenella frontalis.

Despite of the increasing knowledge on sociality and tonal sounds the information remains lacking, or scattered, for many species. Here we are highlighting some of these species, particularly key species in the phylogeny that would 'resolve' the ambiguities observed in the evolution of sociality and tonal sounds.

Pygmy and Dwarf sperm whales (Kogia breviceps and K. sima) [98] are close relatives of the Sperm whale (Physeter macrocephalus) a species that shows a matrilineal society and does not produce tonal sounds. There are no indications that these species show a similar society to that of the Sperm whale. In general their social structure and acoustic signals are poorly known [99-104]. Pygmy and Dwarf sperm whales are often seen and strand in small groups that are can be segregated by age and sex or mixed [102], 
see Table 8]. The few published accounts on their sounds describe click trains $[99,101,103]$ and cry-like sounds [104] but no tonal sounds.

Beaked Whales (Ziphiidae) are largely unknown. The social structure of the Northern Bottlenose Whale (Hyperoodon ampullatus) is the best known of all beaked whales [e.g, 105-109]. The Baird's Beak Whale (Berardius bairdii) is believed to live in stable groups where males may perform parental care [e.g., 86,110, 111]. However, other sources suggest these species live in fission-fusion societies [51]. However both sources report anecdotal evidence and long-term studies are necessary. The social structure of other beaked whales is largely unknown. In terms of tonal sounds, Winn et al. (1970) [112] reported whistles in H. ampullatus, but it appears to be the general consensus that this species does not produce tonal sounds [e.g. 109, Whitehead pers. comn. 2005]. Tonal sounds have been reported as well in the Cuvier's beaked whale, Ziphius cavirostris by Manghi et al. (1999) [113] but other acoustic studies only recorded pulsed sounds [e.g., 114,115]. The only beaked whales for which tonal sounds have been reported are the Baird's Beaked Whale [52] and the Arnoux's Beaked whale (Berardius arnuxii) [51]. There is some possibility that the recordings of Dawson et al. (1998) [52] were of a sympatric dolphin species (Dawson pers. comm.), however, the recordings of Rogers and Brown (1999) [51] seem conclusive.

Inia, Platanista, Lipotes, Orcaella, Neophocaena live in freshwater environments. Generally riverine species are considered solitary, however in some areas these species are often seen forming small groups [see Appendix 1 and respective references]. Although, most authors describe group member interactions in riverine species as weak, there is really little knowledge about their societies. In terms of sound production, like the 
rest of the family (Phocoeenidae) [2], Neophocaena does not produce tonal sounds instead the species emits burst pulses under social context [2]. Tonal sounds have been described for two of the subspecies of Inia geoffrensis, Lipotes vexillifer [see Appendix 7], but not for Pontoporia [116]. Mizue et al. (1971) [117] reported whistles from Platanista gangetica, recorded in captive conditions. However, it is not clear if the animals were acoustically isolated from another riverine dolphin (I. geoffrensis), which produces tonal sounds.

The dolphins Lagenorhynchus cruciger, L. australis, Lissodelphis spp, Steno bredanensis, Feresa attenuata, and Peponocephala electra social structure is largely unknown. Most available information comes from stranding and anecdotic information. Although Fish and Turl (1976) [118] documented whistles in Lissodelphis spp., recent work did not find whistles (Oswald pers. comn). No published accounts on tonal sounds for Feresa and L. cruciger were found. May-Collado and Agnarsson (2006) [58] predict that L. cruciger may not emit whistles as it nests within a clade of species that do not.

\section{Phylogenetic uncertainty}

Taking phylogenetic relationships among species into account is crucial for hypotheses testing in comparative biology. However, this is no simple procedure phylogenies themselves are merely hypotheses and for any given comparative study the number of possible alternative phylogenetic arrangements grows exponentially with the number of species being considered. The key question then becomes, how dependent are our conclusions on the choice of phylogeny? Do the results remain mostly unchangedimplying robustness to phylogenetic uncertainty — or do they change when tests are run 
on alternative "reasonable" phylogenies. Alternative phylogenies can come from several sources, e.g. from previously published independent phylogenetic studies, or from the set of near-optimal trees from a given analysis, e.g. each unique tree from the post burnin set of a Bayesian analysis. If the results of the comparative analyses are different under some of the alternative phylogenies we have not rejected our conclusions but we have been cautioned that the conclusions are dependent on the chosen phylogeny and may be altered as new phylogenetic data become available. If, however, the results are the same across the set of alternative phylogenies then confidence is gained in the conclusions. Here, we attempt to account for phylogenetic uncertainty using various approaches.

The total number of trees in the post-burnin set from the Bayesian analysis is 2000. Instead of basing sensitivity analyses on the $95 \%$ credibility set (which includes a number of trees that contradict all recent studies of whale phylogenetics) we use all the post burnin trees filtered based on various constraints reflecting external phylogenetic evidence. This filtering reduces the number of trees facilitating analyses, without much risk of compromising concerns for phylogenetic uncertainty as the constrained clades are, by any standard, uncontroversial. Rather, considering trees that contradict all available phylogenetic evidence would seem more likely to be misleading than useful. Here, we (1) ran analyzes across the post-burnin set of trees from May-Collado et al. (2007) [59] filtered by constraining major clades all recent phylogenetic studies of Cetacea agree have supported (see below), and (2) using subsets of the post-burnin trees filtered so as to be congruent with other recently published phylogenetic hypotheses of cetaceans chosen as they are based on various types of data: morphological/palaentological (Geisler 2003) [119], mitogenomic (Arnason et al. 2004) [53], a combination of molecular and 
morphological data (Messenger and McGuire 1998) [61] and SINE's (Nikaido et al. 2001) [62]. We chose to use previously published phylogenies as guides to filter trees from the Bayesian post-burnin tree set, rather than to use them directly for analyses (but see below). This is simply because each of these phylogenies contains only a small subset of cetacean species making them poor for the purposes of comparative analyses. Nevertheless, they represent relatively well supported and conflicting hypotheses on the interrelationships of some of the major cetacean clades, whose resolution may impact the findings of our study. Finally, we ran analyses on trees resulting from re-analyses of the Messenger and McGuire dataset, which is the most taxon-rich previously published phylogeny.

We constructed constraint trees in McClade [see Appendix 2] representing each of the previously published phylogeny (see above) and filtered trees from the post-burnin set based on these constraint trees. The constraint trees merely reflect the interrelationships of major clades (families and more inclusive clades, [see Appendix 2]). Species level relationships are not constrained as most of the studies include very few species so that they represent poor tests of lower level phylogenetic structure. Finally, we produced one constraint tree representing only clades that all the previously published studies agree on. This filtering process produced the following datasets: Arnason constraint set (325 trees), Nikaido constraint set (341 trees), Messenger and McGuire constraint set (4 trees), and the all study agreement constraint set (1069 trees). None of the post-burnin trees were congruent with the hypothesis of Geisler (2003) [119]. In fact all other recent molecular, morphological, and combined analyses refute aspects of that hypothesis, in particular the monophyly of all river dolphins (other studies all agree that 
Platanista is not closely related to the remaining river dolphins), and the monophyly of Physeteroidea (other studies refute the sister relationships of Ziphiidae and Physeteridae). Hence we did not further consider that hypothesis, although it played a role in the construction of the 'all study agreement' subset.

SIMMAP analyses were run across all trees in each subset, while PDAP analyses were conducted on the majority rule tree (using contype=allcompat) of each of the subsets. Furthermore, parsimony ancestral character reconstructions were examined on each of the majority rule trees and across all trees from the all study agreement tree subset. 


\section{ACKNOWLEDGEMENTS}

We thank Mike Heithaus, Volker Deecke, and three anonymous reviewers for comments on this manuscript. We also thank Maureen A. Donnelly, Tim Collins, and Zhenim Chen for their support, Tim Collins, Wayne P. Maddison and Peter Midford for advice on some of the analyses and P. Midford for making available to us an unpublished version of PDAP. We thank Julie Oswald and Shannon Rankin for providing us with unpublished acoustic data. Funding for this project came from Judith Parker Travel Grant, LernerGray Fund for Marine Research of the American Museum of Natural History, Cetacean International Society, Latin American Student Field Research Award of the American Society of Mammalogists, the Russell E. Train Education Program-WWF, and FIU Dissertation Year Fellowship, all to Laura May-Collado. This research was in part supported by NSF grant DEB-0516038. 


\section{REFERENCES}

1. Au WWL:Hearing in whales and dolphins: An overview. In: Hearing by Whales and Dolphins. Edited by Au WWL, Popper AN, Fay RR. London: Springer-Verlag Press; 2000: 1-42.

2. Richardson WJ, Green GCJ, Malme CI, Thomsom DH: Marine Mammals and Noise. New York: Academic Press; 1995.

3. Tyack PL: Functional aspects of cetacean communication. In: Cetacean Societies: Field studies of dolphins and whales Edited by Mann J, Connor RC, Tyack PL, Whitehead, H. Chicago: The University of Chicago Press; 2000: 270307.

4. Barklow W: Amphibious communication with sounds in hippos, Hippopotamus amphibius. Animal Behaviour 2004, 68:1125-1132.

5. Clark CW: Acoustic behavior of mysticete whales. In: Sensory abilities of cetaceans. Edited by Thomas J, Kastelein RA. New York: Plenum Press; 1990: 571-583.

6. Oswald JN, Rankin S, Barlow J: The effect of recording and analysis bandwidth on acoustic identification of delphinid species. Journal of the Acoustical Society of America 2004, 116: 3178-3185.

7. Rasmussen MH, Miller LA: Whistles and clicks from white-beaked dolphins, (Lagenorhynchus albirostris Gray 1846) recorded in Faxafloi Bay, Iceland. Aquatic Mammals 2002, 28:78-89.

8. Boisseau $\mathrm{O}$ : Quantifying the acoustic repertoire of a population: the vocalizations of free-ranging bottlenose dolphins in Fiordland, New Zealand. Journal of the Acoustical Society of America 2005, 117:2318-2329.

9. May-Collado LJ, Wartzok D: The freshwater dolphin Inia geoffrensis geoffrensis produces high frequency whistles. Journal of Acoustic Society of America 2007, 121: in press

10. Azevedo AF, Van Sluys M.: Whistles of tucuxi dolphins (Sotalia fluviatilis) in Brazil: comparisons among populations. Journal of the Acoustical Society of America 2005, 117:1456-1464.

11. Barzúa-Durán MC, Au WWL: Whistles of Hawaiian spinner dolphins. Journal of the Acoustical Society of America 2002, 112:3064-3072.

12. Wang X, Wang D, Akamatsu T, Fujita K, Shiraki R: Estimated detection distance of a baiji's (Chinese river dolphin, Lipotes vexillifer) whistles using a passive acoustic survey method. Journal of the Acoustical Society of America 2006, 120:1361-1365.

13. Lammers MO, Au WWL: Directionality in the whistles of Hawaiian spinner dolphins (Stenella longirostris): a signal feature to cue direction of movement? Marine Mammal Science 2003, 19:249-264.

14. Rasmussen MH, Lammers M, Beedholm K, Miller LA: Source levels and harmonic content of whistles in white-beaked dolphins (Lagenorhynchus albirostris). Journal of the Acoustical Society of America 2006, 120:511-517. 
15. Van Parijs SM, Corkeron PJ: Evidence for signature whistle production by a Pacific humpback dolphin, Sousa chinensis. Marine Mammal Science 2001, 17:944-949.

16. Wang D, Wursig B, Evans WE: Whistles of bottlenose dolphins: comparisons among populations. Aquatic Mammals 1995, 21:65-77.

17. Barzúa-Durán MC, Au WWL: Geographic variations in the whistles of spinner dolphins (Stenella longirostris) of the Main Hawaiian Islands. Journal of the Acoustical Society of America 2004, 116:3757-3769.

18. Oswald JN, Barlow J, Norris TF: Acoustic identification of nine delphinids species in the eastern tropical Pacific ocean. Marine Mammal Science 2003, 19:20-37.

19. Rendell LE, Matthews JN, Gill A, Gordon JCD, MacDonald DW: Quantitative analysis of tonal calls from five odontocete species, examining interspecific and intraspecific variation. Journal of Zoology 1999, 249:403-410.

20. Steiner WW: Species-specific differences in pure tonal whistle vocalizations of five western North Atlantic dolphin species. Behavioral Ecological and Sociobiology 1981, 9:241-246.

21. Wang D, Wursig B, Evans WE: Comparisons of whistles among seven odontocete species. In: Sensory Systems of Aquatic Mammals. Edited by R. A. Kastelien, Thomas JA, Nachtigal PE. Woerden. The Netherlands: De Spill Publishers; 1995: 299-323.

22. Wang D, Wursig B, Leatherwwod S: Whistles of boto, Inia geoffrensis, and tucuxi, Sotalia fluviatilis. Journal of the Acoustical Society of America 2001, 109:407-414.

23. Parks SE, Tyack PL: Sound production by North Atlantic right whales (Eubalaena glacialis) in surface active groups. Journal of the Acoustical Society of America 2005, 117:3297-3306.

24. Sirovic A, Hildebrand JA, Wiggins SM, McDonald MA, Moore SE, Thiele D: Seasonality of blue and fin whales calls and the influence of sea ice in the Western Antarctic Peninsula. Deep-Sea Research II 2004, 51:2327-2344.

25. Clark CW, Johnson JH: The sounds of the bowhead whale, Balaena mysticetus, during the spring migrations of 1979 and 1980. Canadian Journal of Zoology 1984, 62:1436-1441.

26. Gedamke J, Costa DP, Dunstan A: Localization and visual verification of a complex minke whale vocalization. Journal of the Acoustical Society of America 2001, 109:3038-3047.

27. Stafford KM, Bohnenstiehl DR, Tolstoy M,Chapp E, Mellinger DK, Moore SE: Antarctic-type blue whale calls recorded at low latitudes in the Indian and eastern Pacific Oceans. Deep-Sea Research II 2004, 51:1337-1346.

28. Watkins WA, Daher MA, George JE, Rodriguez D: Twelve years of tracking 52-Hz whale calls from a unique source in the North Pacific. Deep-Sea Research I 2004, 51:1889-1901.

29. McDonald MA, Hildebrand JA, Wiggins SM, Thiele D, Glasgow D, Moore SE: Sei whale sounds recorded in the Antarctic. Journal of the Acoustical Society of America 2005, 118:3941-3945. 
30. Frankel AS: Sound Production. In: Encyclopedia of Marine Mammals. Edited by Perrin WF, Wursig B., Thewissen JGM. San Diego: Academic Press; 2002: 1126-1137.

31. Reidenberg JS, Laitman JT: Discovery of a low frequency sound source in Mysticeti (Baleen Whales): anatomical establishment of a new vocal fold. Anatomical Record 2007, 290:745-759.

32. Carrington-Stein R: Sound production in Vertebrates: summary and prospectus. American Zoologist 1973, 13:1249-1255.

33. Cranford W: In search of impulse sound sources in Odontocetes. In: Hearing by Whales and Dolphins. Edited by WWL Au, Popper AN, Fay RR. London: Springer Press; 2000.

34. Cranford W, Amundin M, Norris KS: Functional morphology and homology in the odontocete nasal complex: implications for sound generation. Journal of Morphology 1999, 228:223-285.

35. Podos J, Da Silva, VMF, Rossi-Santos MR: Vocalizations of Amazon river dolphins, Inia geoffrensis: insights into the Evolutionary origins of delphinid whistles. Ethology 2002, 108:601-612.

36. Dreher JJ, Evans WE: Cetacean communication. In: Marine Bioacoustics. Edited by Tavolga WN. Oxford: Pergamon Press; 1964.

37. Caldwell MC, Caldwell DK: Individual whistle contours in bottlenose dolphins (Tursiops truncatus). Nature 1965, 207:434-435.

38. Caldwell MC, Caldwell DK, Miller J.F: Statistical evidence for individual signature whistles in the spotted dolphin, Stenella plagiodon. Cetology 1973, 16:1-21.

39. Janik VM, Dehnhardt G, Todt D: Signature whistle variations in bottlenosed dolphin, Tursiops truncatus. Behavioral Ecology and Sociobiology 1994, 35:243-248.

40. Janik VM: Whistle matching in wild bottlenose dolphins (Tursiops truncatus). Science 2000, 289:1355-1357.

41. Herzing DL: Acoustics and social behavior of wild dolphins: implications for a sound society. In: Hearing by whales and dolphins. Edited by Au WWL, Popper AN, RE Fay. London: Springer Press; 2000.

42. Acevedo-Guiterrez A, Stienessen SC: Bottlenose dolphins (Tursiops truncatus) increase number of whistles when feeding. Aquatic Mammals 2004, 30:357362.

43. Fripp D, Owen C, Quintana-Rizzo E, Shapiro A, Buckstaff K, Jankowski K, Wells R, Tyack P: Bottlenose dolphin (Tursiops truncatus) calves appear to model their signature whistles on the signature whistles of community members. Animal Cognition 2005, 8:17-26.

44. Watwood SL, Tyack PL, Wells RS: Whistle sharing in paired male bottlenose dolphins, Tursiops truncatus. Behavioral Ecology and Sociobiology 2004, 55:531-543.

45. Herman LM, Tavolga WN: The communication systems of cetaceans. In: Cetacean Behavior: Mechanisms and Functions Edited by Herman LM. New York: John Wiley Son Publisher; 1980. 
46. Watkins WA, Schevill WE, Best PB: Underwater sounds of Cephalorhynchus heavisidii (Mammalia:Cetacea). Journal of Mammalogy 1977, 58:316-320.

47. Watkins WA, Schevill WE: Characteristic features of the underwater sounds of Cephalorhynchus commersoni. Journal of Mammalogy 1980, 61:738-739.

48. Diazgranados MC, Trujillo F: Vocal repertoire of the freshwater dolphins Inia geoffrensis and Sotalia fluviatilis in Colombia, South America [abstract]. Journal of the Acoustical Society of America 2002, 112:2400.

49. Wang D, Wang K, Akamatsu T, Fujita F: Study on whistles of the Chinese River Dolphin or baiji Lipotes vexillifer. Oceanologia et Limonologia Sinica 1999, 30:349-354.

50. Jing X, Youfo X, Rongcai J: Acoustic signals and acoustic behavior of the Chinese River Dolphin (Lipotes vexillifer). Scientia Sinica 1981, 24:407-415.

51. Rogers TL, Brown SM: Acoustic observations of Arnoux's beaked whale (Berardius arnuxii) off Kemp Land, Antarctica. Marine Mammal Science 1999, 15:192-198.

52. Dawson S, Barlow J, Ljungblad D: Sounds recorded from Baird's beaked whale, Berardius bairdii. Marine Mammal Science 1998, 14:335-344.

53. Watkins WA, Schevill WE, Ray C: Underwater sounds of Monodon (Narwhal). Journal of the Acoustical Society of America 1970, 49:595-599.

54. Ford KB, Fisher HD: Underwater acoustic signals of the narwhal (Monodon monocerus). Canadian Journal of Zoology 1978, 56:552-560.

55. Sjare BL, Smith TG: The vocal repertoire of white whales, Delphinapterus leucas, summering in Cunningham Inlet, Northwest Territories. Canadian Journal of Zoology 1986, 64:407-415.

56. Karlsen JD, Bisther A, Lydersen C, Haug T, Kovacs KM: Summer vocalisations of adult male white whales (Delphinapterus leucas) in Svalbard, Norway. Polar Biology 2002, 25:808-817.

57. Shapiro A: Preliminary evidence for signature vocalizations among freeranging narwhals (Monodon monocerus). Journal of the Acoustical Society of America 2006, 120:1695-1705.

58. May-Collado LJ, Agnarsson A: Cytochrome b and Bayesian inference of whale phylogeny. Molecular Phylogenetics and Evolution 2006, 38: 344-354.

59. May-Collado LJ, Agnarsson I, Wartzok D: Reexamining the relationship between body size and tonal signals frequency in whales: a comparative phylogenetic approach. Marine Mammal Science 2007, 23: 524-552.

60. Muizon $\mathrm{C}$ de: Les relations phylogénétiques des Delphinida. Annales de Paleontologie 1988, 74:157-227.

61. Messenger SL, McGuire JA: Morphology, Molecules, and the Phylogenetics of Cetaceans. Systematic Biology 1998, 47:90-124.

62. Nikaido M, Matsuno F, Hamilton H, Brownell RL Jr, Cao Y, Ding W, Zuoyan Z, Shedlock AM, Fordyce RE, Hasegawa M, Okada N: Retroposon analysis of major cetacean lineages: The monophyly of toothed whales and the paraphyly of river dolphins. $P N A S$ 2001, 98: 7384-7389.

63. Arnason U, Gullberg A, Janke A: Mitogenomic analyses provide new insights into cetacean origna and evolution. Gene 2004, 333: 27-34. 
64. Coddington J: Cladistic tests of adaptational hypotheses. Cladistics 1988, 4:120.

65. Moore SE, Ridgway SH: Whistles produced by common dolphins from Southern California Bight. Aquatic Mammals 1995, 21:55-63.

66. Janik VM, Slate PJB: Context-specific use suggests that bottlenose dolphin signature whistles are cohesion calls. Animal Behaviour 1998, 56:829-838.

67. Sayigh LS, Tyack PL, Wells RS, Scott MD: Signature whistles of free-ranging bottlenose dolphins, Tursiops truncatus: mother-offspring comparisons. Behavioral Ecology Sociobiology 1990, 26:247-260.

68. Dawson SM, Thorpe CW: A quantitative analysis of the sounds of Hector's dolphin. Ethology 1990, 86:131-145.

69. Myrberg AAJr: Sound communication and interception in fishes. In: Hearing and sound communication in fishes. Edited by W. N. Tavolga. London: Springer Press; 1981: 359-426.

70. Deecke VB, Ford JKB, Slater PJB: The vocal behaviour of mammal-eating killer whales: communicating with costly calls. Animal Behaviour 2005, 69:395-405.

71. Watkins WA, Daher MA, Samuels A, Gannon DP: Observations of Peponocephala electra, the Melon-headed whale, in the southeastern Caribbean. Caribbean Journal of Science 1997, 33:34-40.

72. Dudok van Heel WH: Investigations on cetacean sonar. III. A proposal for an ecological classification of cetaceans in relation to sonar. Aquatic Mammals 1981, 8:65-68.

73. Tyack PL: Studying how cetaceans use sound to explore their environment. In: Perspectives in Ethology Edited by Owings DH, Beecher MD, Thompson NS. New York: Plenum Press; 1997: 251-297 [vol. 12].

74. Amundin M: Sound production in odontocetes with emphasis on the harbour porpoise, Phocoena phocoena. Stockholm: University of Stockholm; 1991.

75. Dawson S: Clicks and communication: the behavioral and social contexts of Hector's dolphin vocalizations. Ethology 1991, 88:265-276.

76. Morisaka T, Connor RC: Predation by killer whales (Orcinus orca) and the evolution of whistle loss and narrow-band frequency clicks in odontocetes. Journal of Evolutionary Biology 2007, ??:???-???. [early online access]

77. Caldwell MC, Caldwell DK, Tyack PL: Review of the signature-whistle hypothesis for the Atlantic bottlenose dolphin. In: The bottlenose dolphin Edited by Leatherwood SRRR. San Diego: Academic Press; 1990: 199-234.

78. Caldwell MC, Caldwell DK: Statistical evidence for individual signature whistles in the Pacific whitesided dolphin, Lagenorhynchus obliquidens. Cetology 1970, 16:1-21.

79. Tyack PL: Population biology, social behavior and communication in whales and dolphins. . Trends in Ecology and Evolution 1986, 1(144-149).

80. Tyack PL: Whistle repertoires of two bottlenosed dolphins, Tursiops truncatus: mimicry of signature whistles? . Behavioral Ecology and Sociobiology 1986, 18(251-257). 
81. Tyack PL: Communication and cognition. In: Biology of Marine Mammals Edited by Reynolds JE, Rommen SA. Washington: Smithsonian Institute Press; 1999: 287-323.

82. Sayigh LS, Tyack PL, Wells RS, Scott MD, Irvine AB: Sex difference in signature whistle production of free-ranging bottlenose dolphins, Tursiops truncatus. Behavioral Ecology Sociobiology 1995, 36:171-177.

83. Matthews JN, Rendell LE, Gordon JCD, MacDonald DW: A review of frequency and time parameters of cetacean tonal calls. Bioacoustics 1999, 10:47-71.

84. Ryan MJ: Constraints and patterns in the evolution of anuran acoustic communication. In: The Evolution of the Amphibian Auditory System Edited by Fritzch B, Ryan MJ, Wilczynski W,Walkowiak W, Hetheringon TE. New York: Wiley Press; 1988: 637-677.

85. Thomsen F, Franck D, Ford JKB: Characteristics of whistles from the acoustic repertoire of resident killer whales (Orcinus orca) off Vancouver Island, British Columbia. Journal of the Acoustical Society of America 2001, 109: 12401246.

86. Connor RC, Mann J, Tyack PL, Whitehead H: Social evolution in toothed whales. Trends in Ecology and Evolution 1998, 13:228-232.

87. Connor RC: Group living in whales and dolphins. In: Cetacean Societies: Field studies of dolphins and whales. Edited by Mann J, Connor RC, Tyack PL, Whitehead H. Chicago: The University of Chicago Press; 2000: 199-218.

88. Whitehead $\mathrm{H}$, Bejder L, Ottensmayer A: Testing association patterns: issues arising and extensions. Animal Behaviour 2005, 69:1-6.

89. Karczmarski L, Würsig B, Gailey G, Larson KW, Vanderlip C: Spinner dolphins in remote Hawaiian atoll: social grouping and population structure. Behavioral Ecology and Sociobiology 2005, 16:675-685.

90. Maddison WP, Maddison DR: Mesquite: A modular system for evolutionary analysis.Version 1.12.http://mesquiteproject.org; 2006.

91. Maddison WP: Square-change parsimony reconstructions of ancestral states for continuous-valued characters on a phylogenetic tree. Systematic Zoology 1991, 40:304-314.

92. Felsenstein J: Phylogenies and the comparative method. American Naturalist 1985, 125:1-15.

93. Rohlf FJ: A comment on phylogenetic correction. Evolution 2006, 60:15091515 .

94. Midford PE, Garland TJr, Maddison WP: PDAP Package of Mesquite. http://mesquiteproject.org; 2005.

95. Bollback JP: SIMMAP: Stochastic character mapping of discrete traits on phylogenies. BMC Bioinformatics 2006, 7:1-7.

96. Maddison WP: A method for testing the correlated evolution of two binary characters: are gains or losses concentrated on certain branches of a phylogenetic tree? . Evolution 1990, 44(3): 539-557. 
97. Maddison WP, Maddison DR: MacClade: Analysis of Phylogeny and Character Evolution. Version 4.07. Sunderland, Massachusetts: Sinauer Associates; 2003.

98. Chivers SJ, Leduc RG, Robertson KM, Barros NB, Dizon AE: Genetic variation of Kogia spp with preliminary evidence for two species of Kogia sima. Marine Mammal Science 2005, 21:619-634.

99. Caldwell DK, Caldwell MC: Sounds produced by two rare cetaceans stranded in Florida. Cetology 1971, 1-6.

100. Caldwell DK, Caldwell MC: Pygmy sperm whale Kogia breviceps (de Blainville 1838) and dwarf sperm whale Kogia simus (Owen 1866). In:

Hanbook of Marine Mammals Edited by Ridgway SH, Harrison Sir H, vol. 4. New York: Academic Press; 1989: 235-260.

101. Caldwell DK, Prescott JH, Caldwell DK: Production of pulsed sounds by the pygmy sperm whale, Kogia breviceps. Bulletin Southern California Academy of Science 1966, 65:245-248.

102. McAlpine DF: Pygm and Dwarf sperm whales (Kogia breviceps and $K$. sima). In: Encyclopedia of Marine Mammals Edited by Perrin WF, Wursig B, Thewissen JGM. San Diego: Academic Press; 2002: 1007-1009.

103. Marten K: Ultrasonic analysis of pygmy sperm whale (Kogia breviceps) and Hubb's beaked whale (Mesoplodon carlhubbsi) clicks. Aquatic Mammals 2000, 26:45-48.

104. Thomas JA, Moore PWB, Nachtigall PE, Gilmartin WG: A new sound from a stranded pygmy sperm whale. Aquatic Mammals 1990, 16:28-30.

105. Dalebout MK, Hooker SK, Christensen I: Genetic diversity and population structure among northern bottlenose whales, Hyperoodon ampullatus, in the western North Atlantic Ocean. Canadian Journal of Zoology 2001, 79:478-484.

106. Gowans SL, Rendell, L: Head-butting in northern bottlenose whales (Hyperoodon ampullatus): a possible function for big heads. Marine Mammal Science 1999, 15:1342-1350.

107. Gowans S, Whitehead H, Hooker SK: Social organization in northern bottlenose whales, Hyperoodon ampullatus: not driven by deep-water foraging? Animal Behaviour 2001, 62:369-377.

108. Gowans S, Whitehead H: Photographic identification of northern bottlenose whales (Hyperoodon ampullatus): sources of heterogeneity. Marine Mammal Science 2001, 17:76-93.

109. Hooker SK, Whitehead H: Click characteristics of northern bottlenose whales (Hyperoodon ampullatus). Marine Mammal Science 2002, 18:69-80.

110. Kasuya T: Distribution and behavior of Baird's beake whales off the Pacific coast of Japan. The Scientific Report of Whales Research Institute 1986, 37:6183.

111. Kasuya T, Brownell RLJr, Balcomb IIIKC: Life history of Baird's beaked whales off the Pacific coast of Japan. Reports of the International Whaling Commission 1997, 47:969-979. 
112. Winn HEP, Perkins PJ, Winn L: Sounds and behavior of the northern bottlenose whale [abstract]. In: Proceedings of the 7th Annual Conference on Biological Sonar and Diving Mammals: 1970; Stanford Research Institute, Menlo Park, California; 1970: 52-59.

113. Manghi M, Montesi G, Fossati C, Pavan G, Priano M, Teloni V: Cuvier's beaked whales in the Ionian Sea: First recordings of their sounds. European Research on Cetaceans 1990, 13:39-42.

114. Frantzis A, Goold JC, Skarsoulis EK, Taroudakis MI: Clicks from Cuvier's beaked whales (Ziphius cavirostris). Journal of the Acoustical Society of America 2002, 112:34-37.

115. Zimmer WMX, Johnson MP, Madsen PT, Tyack PL: Echolocation clicks of free-ranging Cuvier's beaked whales (Ziphius cavirostris). Journal of the Acoustical Society of America 2005, 117:3919-3927.

116. Busnell RG, Dziedzic A, Alcuri G: Etudes preliminaries de signoux acoustiques du Pontoporia blainvillei Gervais and d'Orbigny 1844 (Cetacea, Platanistidae). Mammalia 1974, 38: 449-459.

117. Mizue K, Nishiwaki M, Takemura, A: The underwater sound of ganges river dolphin (Platanista gangetica). The Scientific Report of Whales Research Institute 1971, 23:123-128.

118. Hay, KA, Mansfield AW: Narwhal, Monodon monocerus (Linnaeus 1758). In: Hanbook of Marine Mammals Edited by Ridgway SH, Harrison Sir H, vol. 4. New York: Academic Press; 1989: 145-176.

119. Fish JF, Turl CW: Acoustic source levels of four species of small whales. In: Naval Undersea Center Technical Report 1976: 1-14. 
Table 8 . Definitions of sociality and tonal sound characters and respective states

\begin{tabular}{|c|c|c|c|c|}
\hline \multicolumn{5}{|c|}{ SOCIALITY-BROAD CONCEPT APPROACH } \\
\hline CHARACTER/STATES & $\mathbf{0}$ & 1 & 2 & 3 \\
\hline SOCIALITY & $\begin{array}{l}\text { Species do not live in groups. } \\
\text { Mainly found singly or in pairs. } \\
\text { Pairs are primarily mother with } \\
\text { their calf. Sometimes groups may } \\
\text { form but these are temporal (e.g., } \\
\text { breeding, feeding, or migration) } \\
\text { and do not show any social } \\
\text { structure apart from that of mother } \\
\text { and calf }\end{array}$ & $\begin{array}{l}\text { Group living species. In addition to } \\
\text { mother and calf associations animals are } \\
\text { continuously associating with other } \\
\text { conspecific. These associations may be } \\
\text { short or long-term. Animals within a } \\
\text { group may or not be related. Living } \\
\text { singly is extremely rare within this } \\
\text { species and it is probably limited to old } \\
\text { or outcast animals. }\end{array}$ & & \\
\hline SOCIAL STRUCTURE & $\begin{array}{l}\text { Solitary species with strong social } \\
\text { bonds limited to the time the calf is } \\
\text { dependent of the mother. Animals } \\
\text { may aggregate for breeding, } \\
\text { feeding, or migration but } \\
\text { associations are limited to the } \\
\text { duration of these periods. Groups } \\
\text { are not socially structured }\end{array}$ & $\begin{array}{l}\text { Group living species where all group } \\
\text { members show weak or fluid } \\
\text { associations. Both sexes disperse from } \\
\text { natal group. }\end{array}$ & $\begin{array}{l}\text { Group living species. Group } \\
\text { members show fluid } \\
\text { associations but may have long- } \\
\text { term associations with specific } \\
\text { group members that are not } \\
\text { close relatives e.g, male } \\
\text { alliances and coalitions. Both } \\
\text { sexes disperse from natal group. }\end{array}$ & $\begin{array}{l}\text { Group living species. Group } \\
\text { members are close relatives. } \\
\text { Natal philopatry is sex } \\
\text { dependent but in some } \\
\text { species there is no } \\
\text { dispersion. Long-term } \\
\text { associations. }\end{array}$ \\
\hline \multicolumn{5}{|c|}{ SOCIALITY-MULTI COMPONENT APPROACH } \\
\hline Group Type & $\begin{array}{l}\text { Species described as largely } \\
\text { solitary, but that are often found in } \\
\text { pairs (mother-calf) }\end{array}$ & $\begin{array}{l}\text { Group living species that are generally } \\
\text { found in small groups }\end{array}$ & $\begin{array}{l}\text { Group living species that are } \\
\text { generally found in medium to } \\
\text { large size schools }\end{array}$ & \\
\hline $\begin{array}{l}\text { Group } \\
\text { Stability/Associations }\end{array}$ & $\begin{array}{l}\text { Short when found in non-socially } \\
\text { structured groups. Limited to the } \\
\text { time the calf is dependent of the } \\
\text { mother. }\end{array}$ & $\begin{array}{l}\text { Species where group stability is short. } \\
\text { Animals join and leave the group through } \\
\text { the day. Described in literature as fluid } \\
\text { societies. }\end{array}$ & $\begin{array}{l}\text { Species with fluid societies but } \\
\text { were some conspecific group } \\
\text { show relatively long lasting } \\
\text { associations e.g., male alliances, } \\
\text { female nurseries }\end{array}$ & $\begin{array}{l}\text { Species that live in their } \\
\text { natal group for life. } \\
\text { Animals are related to } \\
\text { group members and } \\
\text { dispersal is limited showing } \\
\text { long-lasting associations }\end{array}$ \\
\hline Group Composition & Mother and calf & Segregated by age and sex & $\begin{array}{l}\text { Mixed (contain both sexes and } \\
\text { several ages) }\end{array}$ & $\begin{array}{l}\text { Both segregated and mixed } \\
\text { (state only used for the test } \\
\text { of association not for } \\
\text { optimizations) }\end{array}$ \\
\hline \multicolumn{5}{|c|}{ TONAL SOUND COMPLEXITY DISCRETE APPROACH } \\
\hline $\begin{array}{l}\text { Tonal Sound } \\
\text { Complexity (2-state) }\end{array}$ & $\begin{array}{l}\text { Mean inflection point is less or } \\
\text { equal to } 1\end{array}$ & Mean inflection point is more than 1 & & \\
\hline $\begin{array}{l}\text { Tonal Sound } \\
\text { Complexity (2-state) }\end{array}$ & $\begin{array}{l}\text { Mean inflection point is between } 0 \text { - } \\
1\end{array}$ & Mean inflection point is between $1.1-2$ & $\begin{array}{l}\text { Mean inflection point is } \\
\text { between } 2.1-3\end{array}$ & $\begin{array}{l}\text { Mean inflection point is } \\
\text { more than } 3.1\end{array}$ \\
\hline
\end{tabular}


Table 9. Probabilities of association between sociality (selecting the highest social state for polymorphic species) and tonal sound complexity. Significant positive associations at p-values $>0.972$ and $0.973 * *$ for two and four state complexity characters, respectively and significant negative associations at $\mathrm{p}$-values $<0.028$ and $0.027^{*}$ for two and four state complexity characters, respectively

\begin{tabular}{|c|c|c|c|c|}
\hline \multirow{2}{*}{$\begin{array}{c}\text { TONAL SOUND } \\
\text { COMPLEXITY [TWO \& FOUR STATE } \\
\text { CHARACTER] }\end{array}$} & \multicolumn{4}{|c|}{$\begin{array}{c}\text { SOCIAL STRUCTURE [FOUR STATE } \\
\text { CHARACTER] }\end{array}$} \\
\hline & 0 & 1 & 2 & 3 \\
\hline $\begin{array}{c}0(\leq 1 \text { mean inflection point }) \\
D \text {-statistic } \\
p \text {-value }\end{array}$ & $\begin{array}{c}0.0821 \\
0.798 \\
\end{array}$ & $\begin{array}{c}0.0536 \\
0.728 \\
\end{array}$ & $\begin{array}{c}-0.0424 \\
\mathrm{p}<0.0001 *\end{array}$ & $\begin{array}{l}-0.0047 \\
0.003^{*}\end{array}$ \\
\hline $\begin{array}{c}\text { ( } \leq 1 \text { mean inflection point }) \\
D \text {-statistic } \\
\text { p-value }\end{array}$ & $\begin{array}{c}-0.0440 \\
\mathrm{p}<0.0001^{*}\end{array}$ & $\begin{array}{c}0.00045 \\
0.90\end{array}$ & $\begin{array}{c}0.113 \\
0.99 * *\end{array}$ & $\begin{array}{l}0.0360 \\
0.99 * *\end{array}$ \\
\hline $\begin{array}{c}0(0-1) D \text {-statistic } \\
\text { p-value }\end{array}$ & $\begin{array}{c}0.084 \\
0.93\end{array}$ & $\begin{array}{c}-0.00029 \\
0.055\end{array}$ & $\begin{array}{l}-0.0338 \\
0.002 *\end{array}$ & $\begin{array}{c}0.009 \\
0.88\end{array}$ \\
\hline $\begin{array}{c}1(1.1-2) \text { D-statistic } \\
\text { p-value }\end{array}$ & $\begin{array}{l}-0.038 \\
0.002 *\end{array}$ & $\begin{array}{c}0.027 \\
0.91\end{array}$ & $\begin{array}{c}0.0781 \\
0.92\end{array}$ & $\begin{array}{c}0.022 \\
0.92\end{array}$ \\
\hline $\begin{array}{c}2(2.1-3) \text { D-statistic } \\
\text { p-value }\end{array}$ & $\begin{array}{l}-0.003 \\
0.018^{*}\end{array}$ & $\begin{array}{c}0.0121 \\
0.89 \\
\end{array}$ & $\begin{array}{c}0.0198 \\
0.91 \\
\end{array}$ & $\begin{array}{l}-0.0033 \\
0.014^{*}\end{array}$ \\
\hline $\begin{array}{c}3(>3.1) \text { D-statistic } \\
\text { p-value }\end{array}$ & $\begin{array}{l}-0.0046 \\
0.012 *\end{array}$ & $\begin{array}{c}0.0151 \\
0.90\end{array}$ & $\begin{array}{c}0.0065 \\
0.86\end{array}$ & $\begin{array}{c}0.0023 \\
0.84\end{array}$ \\
\hline
\end{tabular}


FIG. 11

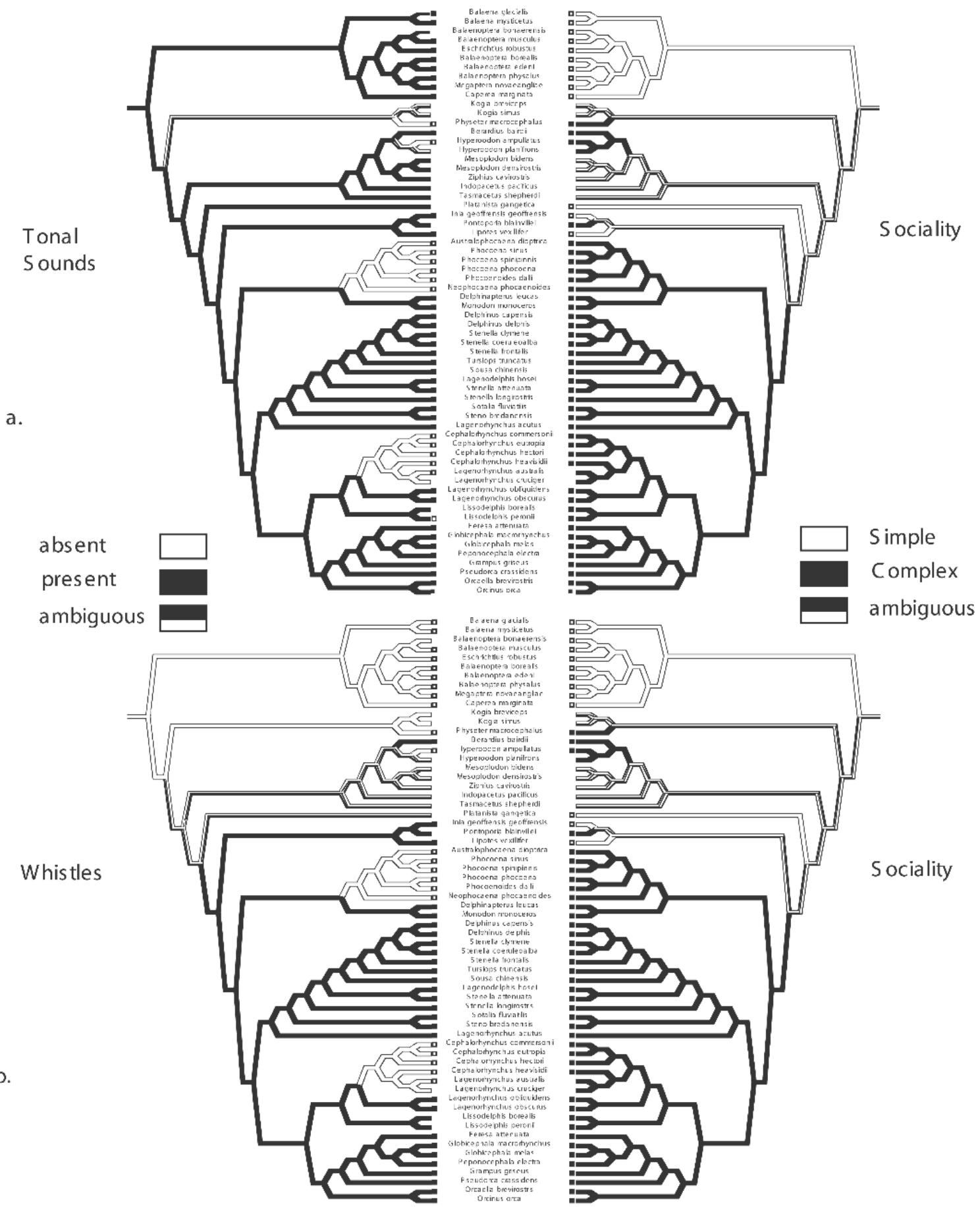


FIG. 12

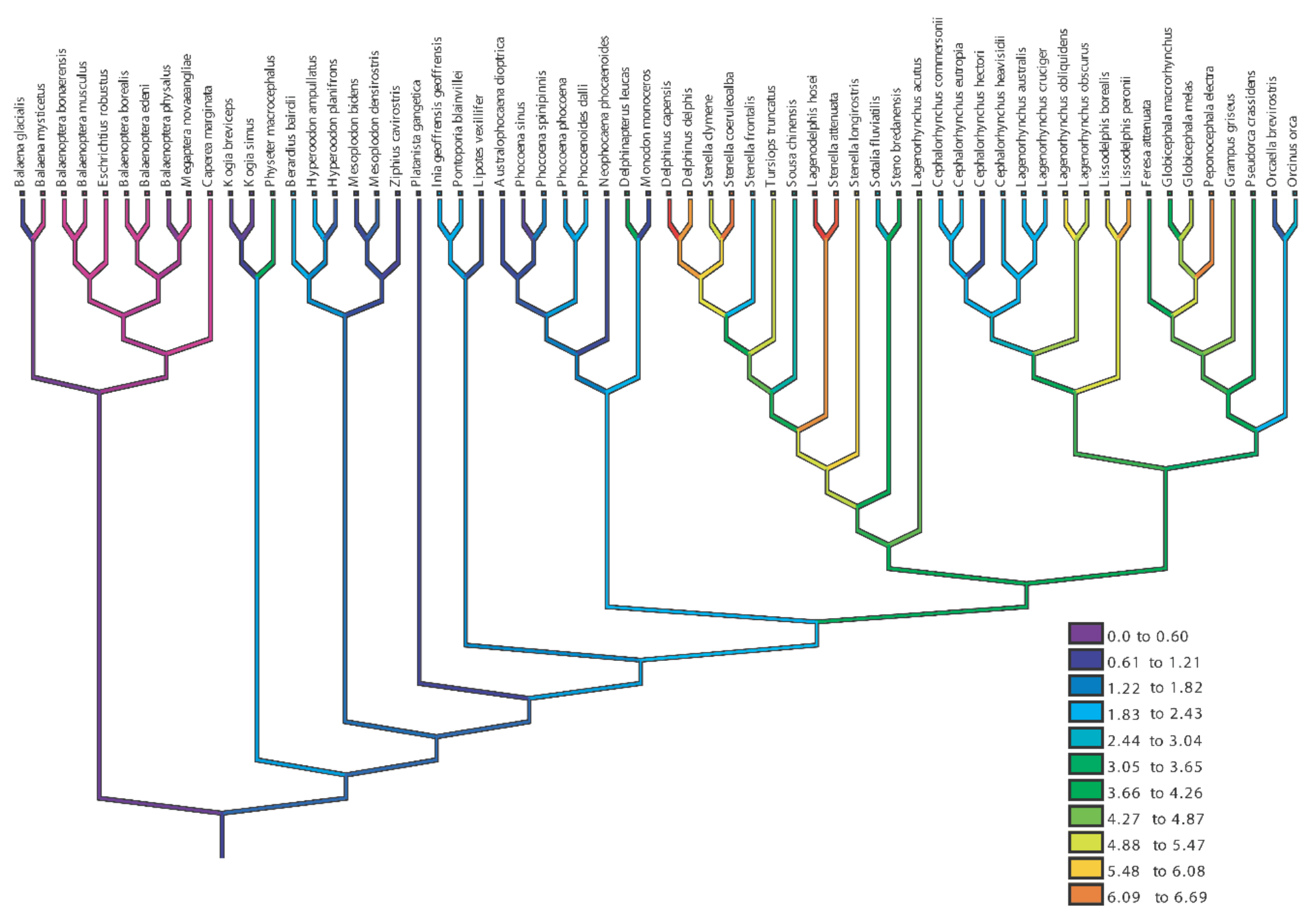


FIG. 13
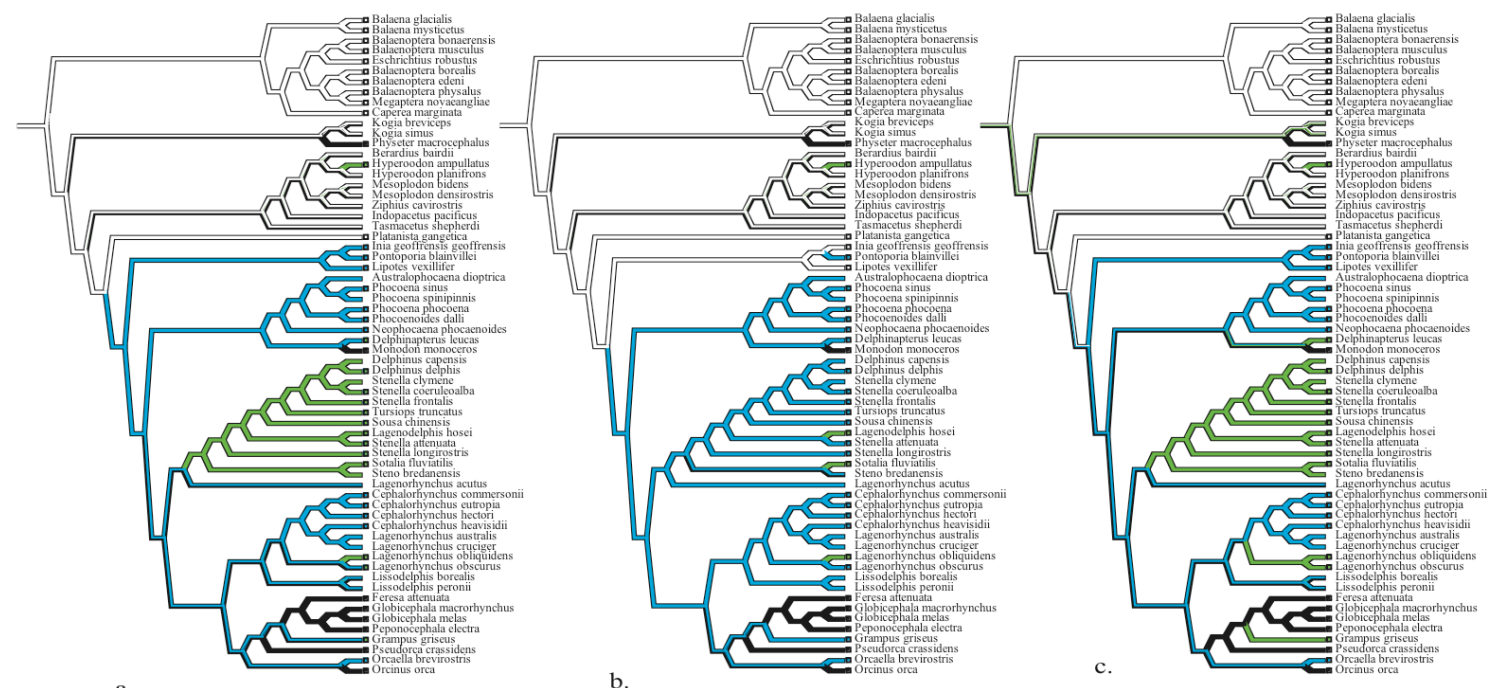

Live solitary/pairs (mother+calf)

Group living with weak associations

Group living with weak /longassociations

Group living long/animals related 
FIG. 14
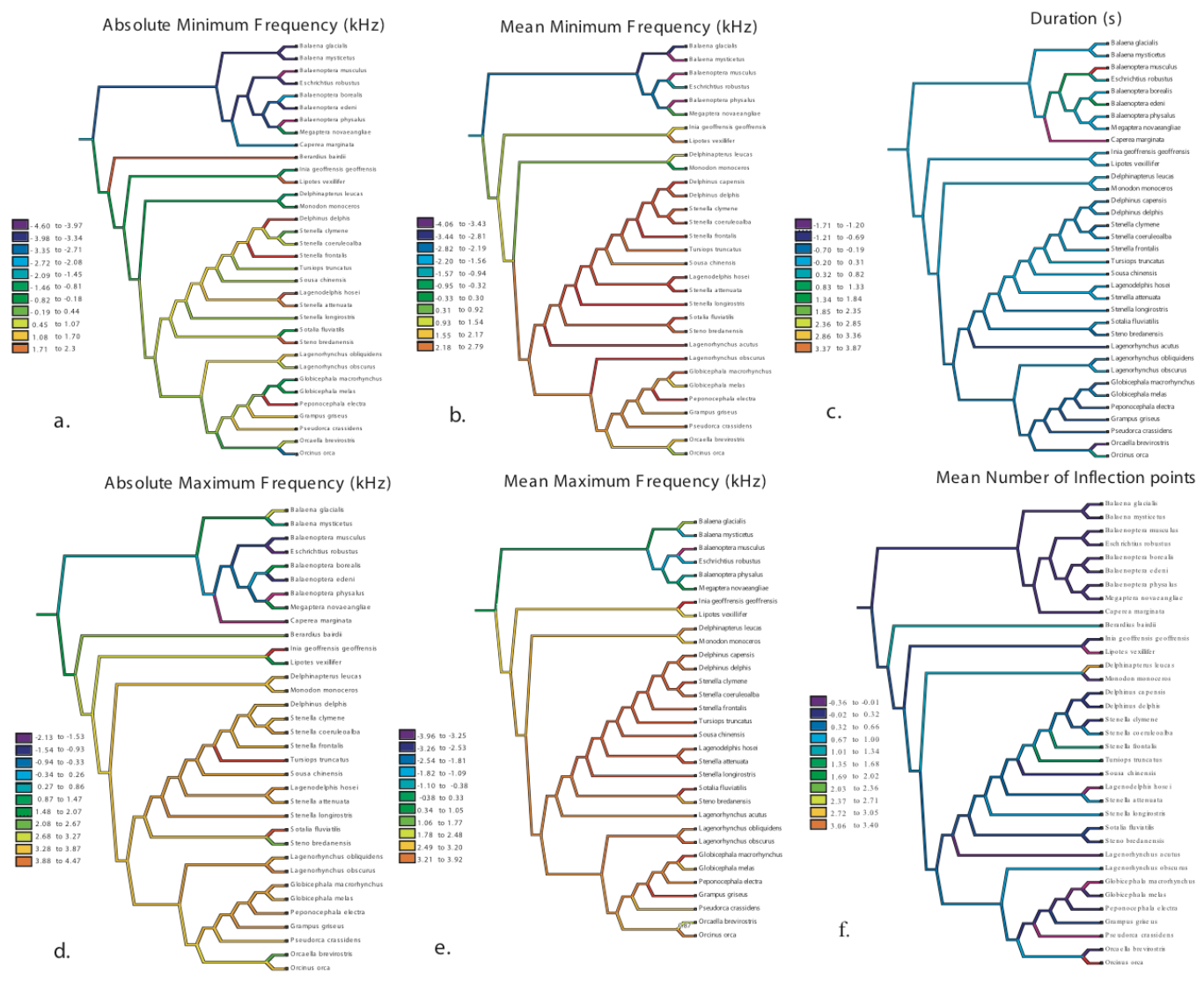

Mean Maximum Frequency ( $\mathrm{kHz}$ )
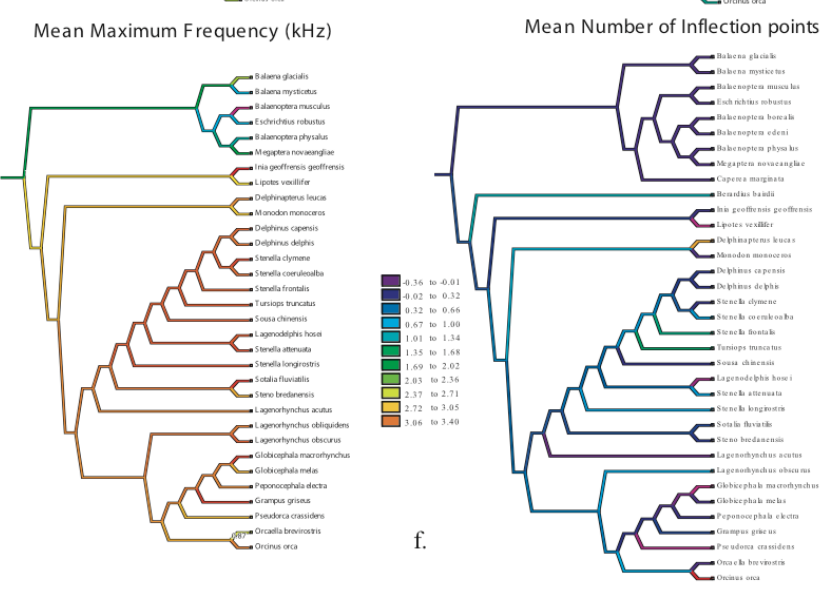
FIG. 15

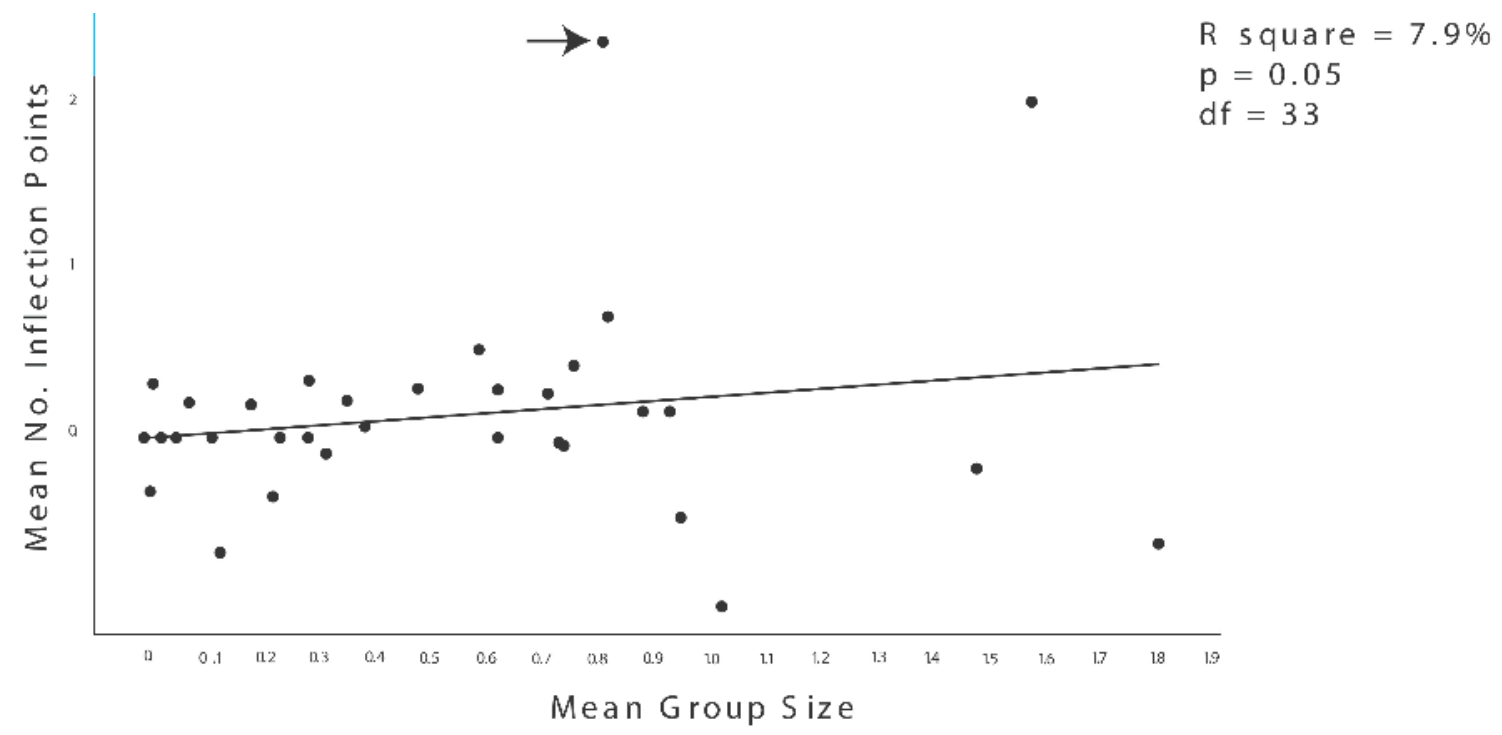




\title{
CHAPTER VI
}

\section{GEOGRAPHICAL WHISTLE VARIATION BETWEEN ADJACENT BOTTLENOSE DOLPHIN POPULATIONS}

\author{
LAURA J. MAY-COLLADO ${ }^{1,2}$ AND DOUGLAS WARTZOK ${ }^{1}$ \\ ${ }^{1}$ Florida International University, Department of Biological Sciences, $11200 \mathrm{SW} 8^{\text {th }}$ \\ Street, Miami, FL 33199, USA \\ ${ }^{2}$ Universidad de Costa Rica, Escuela de Biología, Apto. 2060 San Pedro, Costa Rica
}




\begin{abstract}
Whistles are narrowband and frequency modulated sound produced by many cetaceans. These sounds have been extensively studied in delphinids. Several factors have been proposed to explain between- and within-species variation in whistles. This study aims to bring insight in micro-geographic whistle variation of two bottlenose dolphin populations (Bocas del Toro, Panama vs. Gandoca Manzanillo, Costa Rica) by assessing several factors e.g., habitat acoustic characteristics (ambient and anthropogenic), sympatry with other dolphin species, and intrinsic differences between populations due to variation in behavioral activities and/or distance between populations in some cases leading to isolation. Our results show that the two adjacent populations are distinct in both frequency and temporal whistle parameters. The differences in the mean values of each whistle parameter between these adjacent populations were no smaller than differences between those and more distant populations in the north and south. There were no apparent trends in increasing or decreasing whistle frequency with latitude as shown for other dolphin species. We found that a combination of factors may contribute to the significant differences found between these two adjacent ( $\sim 35 \mathrm{~km}$ apart) populations. Isolation or relatively low mixing of populations may be important. An ongoing photo-ID of these dolphins has hitherto not found any matches between populations. The acoustical structure of their habitat also may play a role. Bocas del Toro has a higher boat traffic rate but lower overall low frequency ambient noise levels. Dolphins produced whistles with characteristics that may help them to cope with their respective environmental noises. While dolphins from Bocas emit longer whistles (maybe to avoid masking by high-frequency boats), dolphins from Gandoca-Manzanillo emit high
\end{abstract}


frequency whistles (perhaps to avoid masking by low frequency ambient noise levels). Sympatry with Sotalia guianensis also may be a factor. However, if this sympatry has an effect, it is in the opposite direction than predicted by the 'species hypothesis'. Bottlenose dolphins sympatric with Sotalia produce whistles that are more similar to Sotalia whistles than are the whistles of non-sympatric bottlenose dolphins. Finally, behavior may be an important source of within population variation. Populations may vary in their investment in different activities. The results of this study suggest that bottlenose dolphin whistles are plastic and influenced by a variety of factors. Isolated populations can be expected to be locally adapted and thus differ from other isolated populations.

KEY WORDS: ambient noise, isolation, boat traffic, behavior, zoogeography, sympatry 


\section{INTRODUCTION}

Most toothed whales emit frequency modulated tonal sounds that are narrowband in frequency (with most of their energy below $20 \mathrm{kHz}$ ) (Au 2000, Richardson et al. 1995). These signals are produced under a variety of social contexts. In true dolphins (Delphinidae), tonal sounds are typically referred to as whistles, and are emitted especially during social interactions that involve group cohesion, individual recognition, and recruitment during feeding activities (e.g. Caldwell and Caldwell 1965, 1990, Sayigh et al. 1995, Janik et al. 1994, Janik 2000, Acevedo-Gutierrez and Stienessen 2004).

Whistle interspecific variation occurs primarily in frequency variables (e.g., Steiner 1981, Wang et al. 1995b, Rendell et al. 1999, Matthews et al. 1999). Several factors have been proposed to explain frequency variation across species, including phylogeny, sociality, zoogeography, and morphological constraints. Recent comparative phylogenetic studies May-Collado et al. (2007a-b) examined the evolution of some frequency components in Cetacean tonal sounds. Their findings suggest that the evolution of minimum frequency in Cetaceans appears to be influenced by body size and group size, whereas whistle complexity (measured in terms of mean number of inflection points) was influenced by social structure.

Whistle variation also occurs within species. Several studies have found variation in frequency modulation (mean number of inflection points) and whistle duration (e.g., Wang et al. 1995a, Azevedo et al. 2005, Morisaka et al. 2005a). However, recent studies have also found frequency parameters as key to discriminate between populations (e.g., Morisaka et al. 2005a, Azevedo et al. 2005, Rossi-Santos and Podos 2006). Such differences have been observed both at micro-geographic scales (between neighboring 
populations) and macro-geographic scales, between widely separated ones (e.g., Wang et al. 1995a, Barzua-Duran and Au 2002, 2004, Azevedo and Sluys 2005, Morisaka et al. 2005a, Rossi-Santos and Podos 2006, Azevedo et al. 2007, Camargo et al. 2007, dos Santos et al. 2007, May-Collado and Wartzok 2007). The general pattern suggests greater whistle variation between populations that are further apart (e.g., Wang et al. 1995a, Barzua-Duran and Au 2002, 2004, Azevedo and Sluys 2005, Rossi-Santos and Podos 2006). However, a recent study in spinner dolphins found that some populations from the Atlantic and Pacific where more similar than less distant populations (Camargo et al. 2007).

In many terrestrial animals (particularly birds) geographic variation in signal structure has provided insights into the dispersal capabilities of species (e.g., Mundinger 1982; McGregor et al. 2000), isolation and genetic divergence between groups or populations (e.g., Lemon 1966, McGregor et al. 2000, Ford 2002), and adaptation to ecological conditions (e.g., Marler 1960, Brumm 2006, Boncoraglio and Saino 2007, Gillam and McCracken 2007, Peters et al. 2007).

The causes of geographical variation in dolphins (and cetaceans in general) are still poorly known. However, recent studies suggest that the acoustic structure of the habitat (which is described in terms of ambient noise, known sources of anthropogenic noise, and the physical structure e.g., bottom substrate, currents, etc) may play an important role in the reported intra-specific whistle variation. Morisaka et al (2005b) found that Indo-Pacific bottlenose dolphins (Tursiops aduncus) from three populations around Japan differ in their whistle frequency structure (adopted frequencies and coefficient of frequency modulation). Dolphins from the noisiest habitat tended to 
produce low frequencies whistles with little modulation as potential strategy to avoid masking and attenuation of higher-frequency signals (Morisaka et al. 2005b). In contrast, short-beaked common dolphins (Delphinus delphis) from the English Channel British Isles, whistled at higher frequency when compared with common dolphins from the Celtic Sea (Ansmann et al. 2007). In this case, dolphins emitted high-frequency whistles in the presumably noisiest site (no measurements of ambient noise were taken), the English Channel, where dolphins may avoid masking by the low-frequency ambient noise produced by the high vessel traffic of the area.

Other proposed factors for whistle intraspecific variation include recent isolation events (e.g., in spinner dolphins see Camargo et al. 2007), intra-specific variation in group fluidity or group stability in association patterns (e.g., in spinner dolphins of Hawaii see Barzua-Duran and Au 2002), and zoogeographical relationships (Steiner 1981). Steiner (1981) suggested that sympatric dolphin species would tend to be more different than when they occur separately.

The goal of this study is to evaluate the interaction of some of these factors and whistle acoustic variation between two adjacent populations of bottlenose dolphins (Tursiops truncatus) in the southern Caribbean of Central America. We tested the following factors: ambient noise levels, boat traffic, sympatry with the coastal Guyanese dolphin (Sotalia guianensis), and intrinsic population in terms of differences due to behavioral states and degree of isolation (distance) by comparing the whistle parameters of these populations with populations in the western north and southern Atlantic. 


\section{MATERIAL AND METHODS}

\section{Study areas and dolphin populations}

The study took place in Gandoca-Manzanillo Wildlife Refuge from 2004 to 2007 and the Archipelago of Bocas del Toro in 2004, 2006, and 2007. Gandoca-Manzanillo Wildlife Reserve is located along the Caribbean coast of Costa Rica, about $35 \mathrm{~km}$ north of the Archipielago Bocas del Toro. The Refuge was established in 1985 and includes about $38.33 \mathrm{~km}$ of land and sea. It was primarily established to protect coral reefs, mangroves, swamps, and flooded forest. Boat traffic is relatively low. Powered boats are used in the Refuge for local fishing and tourism interested in sport fishing (at the mouth of the Sixaola River) and dolphin watching. Dolphin watching is boat based, and possibly the main reason for boat traffic during high tourist season. There are two small resident populations of dolphin species, the Guyanese dolphin (Sotalia guianensis) and the bottlenose dolphin (Tursiops truncatus). The species are sympatric within the limits of the Refuge, where they form mixed-species groups regularly (Acevedo et al. 2005, Gamboa-Poveda and May-Collado 2006). Preliminary photo-ID suggests that only a part of the identified bottlenose dolphins are resident to the Refuge, most appear to have a more offshore range (May-Collado et al. unpublished data).

The Province of Bocas de Toro, Panama covers about $8,745 \mathrm{~km}$. The province consists of several islands including the main island Isla Colon the protected island of Bastimento (under the category of National Park), mainland of Almirante Bay, where the main port of the area is located, Bocas Torito, Tierra Oscura, Punta Laurel, and Cauchero. Some of the islands of the archipelago are somewhat interconnected with the mainland by islets of mangroves (Fig. 10). The main way of transportation between the 
islands and mainland are powered boats and canoes. In Bocas the resident bottlenose dolphin population is small. Unlike Gandoca-Manzanillo most of the identified dolphins are regularly seen in the area, and also show strong site fidelity within the Archipelago (May-Collado et al. unpublished data). No other dolphin species are found in the area. Despite the relatively short distance between the two study areas $(35 \mathrm{~km})$ there is no evidence of mixing between the two populations after four years of ongoing research (May-Collado personal observation).

\section{Recordings}

Signals were recorded using a broadband system consisting of a RESON hydrophone ($203 \mathrm{~dB}$ re $1 \mathrm{~V} / \mu \mathrm{Pa}, 1 \mathrm{~Hz}$ to $140 \mathrm{kHz}$ ) connected to AVISOFT recorder and Ultra Sound Gate 116 (sampling rate $400-500 \mathrm{kHz} 16$ bit) that sent the signals to a laptop.

Ambient noise was recorded in five stations in Bocas del Toro and in three in Gandoca-Manzanillo (see Fig.16) at 500 and 384 kHz sampling rate. One-minute ambient noise files were recorded every five minutes in a period of 15 minutes at each station and at a known gain level. To calibrate ambient noise level recordings we used a calibrated ITC-1001 sound projector to send 2, 6, 10,14, 18, and $22 \mathrm{kHz}$ sine waves to the recording system. Projector and hydrophone were at distance of $7.3 \mathrm{~m}$. The rms voltage input to the ITC-1001 was measured at each frequency and the received sound level at $7.3 \mathrm{~m}$ was calculated based on spherical spreading. We randomly selected $1 \mathrm{sec}$ of the 'control' (each of the above frequencies) and join it with $1 \mathrm{sec}$ segment separately with each of the three recorded files with ambient noise (three $2 \mathrm{sec}$ files) of same sampling rate (500 $\mathrm{kHz}$ ). For ambient noise files with $384 \mathrm{kHz}$ sampling rate we selected $1.3 \mathrm{sec}$, to 
compensate for differences in sampling rate with the control $(500 \mathrm{kHz})$, so that both files had the same number of points rather than the same length of time. Each control $1 \mathrm{sec}$ file was joined separately with $1 \mathrm{sec}($ or $1.3 \mathrm{sec}$ ) ambient noise using the software Media Join 1.0 (Mystik Media (C 2004-2005). Later the joined files were opened in RAVEN PRO 1.3 beta version build 20 (Cornell Lab of Ornithology 2003-2007 (C). The average relative power in $\mathrm{dB}$ for the one second of control and one second of ambient noise were measured. Although RAVEN provides only relative, not absolute, power levels, we knew the actual recorded levels in the control segments and could then calculate the levels of ambient noise.

Dolphin whistles were recorded continuously with a sampling rate between $384-$ $500 \mathrm{kHz}$. For accompanying behavioral observations, recording sessions were segmented into three-minute intervals. Behavioral observations were made every other interval (group scanning lasted two-three minutes as well) and the predominant behavior during that interval was recorded. The predominant behavior was decided based on the activity of most members in a group. A group was defined as all group members maintaining a distance no more than 10 times their body length and engaged in similar behavioral activities (Smolker et al. 1992). However, when several groups were present and relatively close to each other, these were considered the same 'acoustic' group for that particular recording session. This is because we could not associate the recorded signals with their respective group. In this case, the predominant behavior was assigned based on the most common behavior or behaviors in which all groups were engaged. When animals were performing a behavior difficult to assign to any of the below defined categories 
during a scanning period, the behavior was noted as unknown. Five behavioral categories were defined based on a combination of previous definitions (e.g., Lusseau and Higham 2004, May-Collado and Morales-Ramirez 2005):

(1) Feeding/Foraging/Diving: individuals actively searching, pursuing, and/or consuming prey were assigned to this category. Often diving periods were long and involved 'steep dives' where animals arched their backs and lifted the tail vertically before each dive. Direction and distance between animals varied depending on individual or apparent group feeding. (2) Social activities: dolphins interacted among themselves e.g., different types of body contact (aggressive and 'friendly'), tail slapping, and animals following the boat or other dolphins. Groups tend to split in small subgroups and sometimes spread over a larger area, or the opposite when small groups reunite in bays and form temporarily large groups. (3) Traveling: dolphins swimming either slowly or fast but always maintaining a steady direction, (4) Resting: dolphin swimming at a slow speed, surfacing regularly and often synchronically but always within a small area. Often but not always group members where close to each other. (5) Milling: group members spaced and showing random directionality during swimming, often slowly and within the same area. Surfacing patterns were variable. (6) Unknown: assigned to groups when several factors, such as weather condition, and uncertainty due to elusive behavior by the dolphins would not allow the predominant behavior to be clearly determined.

Whistles were analyzed in RAVEN 1.2 (Cornell Lab of Ornithology 2003-2007 (C) with a FFT size of 1024 points, an overlap of 50\%, and using a 512-522 sample Hann window. Nine standard parameters were measured for every highly quality whistle (the entire contour was clearly seen): beginning frequency (Beg), ending frequency (End), 
minimum frequency (Min), maximum frequency (Max), delta frequency (MaxF-MinF), peak frequency (measured in the whistle contour were intensity was the highest), duration (s), number of inflection points, and number of harmonics. In addition, we followed Morisaka et al. (2005b) study by measuring adopted frequencies (McCowan 1995) in order to measure the frequency distribution of a whistle. Nineteen intervals equally distanced were set in every whistle by diving is duration by 20 frequency points (McCowan 1995). These same adopted frequencies were used to calculate a coefficient of frequency modulation (COFM) for each whistle (McCowan and Reiss 1995). The coefficient measures changes in complexity of whistle contour and represents the magnitude of frequency modulation in a whistle. High COFM indicate high frequency modulation (see Morisaka et al. 2005b).

$$
\mathrm{COFM}=\sum_{(n=1,19)}\left|\mathrm{Y}_{n+1}-\mathrm{Y}_{n}\right| / 10,000,
$$

Where $Y_{n}$ is the frequency at the $n$th frequency point.

\section{Boat traffic}

Boat sighting rate during dolphin encounters for Bocas and Gandoca-Manzanillo has been estimated as 0.66 and 0.21 boat $/ \mathrm{min}$ (Taubitz 2007). In Bocas a boat is in sight every 1.5 minutes while in Gandoca-Manzanillo every 4.8 minutes (Taubitz 2007). In Bocas boats are used for local transportation, personal, fishing, and for dolphin watching activities and in Gandoca-Manzanillo for local fishing and tourist activities like sport fishing and dolphin-watching. The majority of boats in Bocas are powered with engines between 50-150 hp while in Gandoca-Manzanillo the majority of the boats use engines 
less than $50 \mathrm{hp}$ (Taubitz 2007). The presence/absence of boats was noted during the recording sessions. When boats were present we also noted the number of boats present in each recording file. The absence of boats in this study refers to the presence of only our research boat. Boat presence was considered as such when a boat was in view within a maximum distance of $500 \mathrm{~m}$ or when it was acoustically detectable by our recording equipment (and thus possibly within the acoustic range of the dolphins).

\section{Statistical analysis}

The Median nonparametric test, run in JMP 7.0 (SAS Institute Inc. 2007) was used to compare the overall median noise levels between sites and within sites. Standard whistle parameters (Min, Max, Delta, Beg, End, Peak, duration, number of inflection pointes and harmonics), the coefficient of modulation and adopted frequencies were compared between populations using the nonparametric test Mann-Whitney U in SYSTAT 12.0 software (SYSTAT Software, Inc. 2007). With the exception of the variables number of inflection points and harmonics, all whistle parameters were Box-Cox transformed to adjust their distribution to nearly normal (Sokal and Rohlf 1995). Then we (1) compared the coefficient of frequency modulation considering the effect of population, whistle duration and their interaction, and (2) we tested population, behavior, boat presence, and site fidelity (in the case of Bocas dolphins) as factors and evaluate their explanatory power and their interaction when comparing all whistle parameters between populations by analysis of covariance (ANCOVA) in the JMP software (SAS Institute Inc. 2007). The transformed variables were also evaluated using a multivariate discriminant function analysis (with a discriminant linear method) to classify whistles within and between 
populations and between and within species (using the software JMP 7.0). Within population whistle variation was evaluated across behavioral states and boat presence/absence using the nonparametric tests Kruskal-Wallis and Mann-Whitney U, respectively. Because dolphins in Bocas appear to show strong site fidelity we evaluated if whistle parameters vary across sites using the above statistical tests. Finally, we test if the overlap in home ranges between the bottlenose dolphins and Sotalia guianensis from Gandoca-Manzanillo influences whistle variation. In other words is whistle variation between bottlenose dolphins and Sotalia from Gandoca-Manzanillo larger than the variation between Sotalia and bottlenose dolphins from Bocas. We tested if the differences in means were significantly different between sympatric and non-sympatric dolphins with a $\chi^{2}$ test.

Finally, we compare the mean values of whistle parameters from our results with other studies by first testing for homogeneity of variances (Levene's F test) and then used a t-test (when variances were equal) or Welch t-test (when variances were unequal). We also tested if the differences in the mean of the various whistle parameters were significantly different between adjacent and the different distant populations with oneway $\chi^{2}$ test. We tested for the two adjacent populations the hypothesis, Ho=no significant difference, and expected values were calculated dividing the total by the number of parameters being compared). 


\section{RESULTS}

Comparisons between adjacent populations: A discriminant analysis misclassified only $25.37 \%$ of the whistles. A total of 103 out of 128 whistles were correctly assigned to the Bocas population, and 50 out of 77 whistles to Gandoca-Manzanillo. Whistle standard parameters differ significantly between dolphin populations, with the exception of minimum and peak frequency, mean number of harmonics, and the coefficient of modulation (see Table 10 for $p$-values). In general, Bocas dolphin whistled with lower maximum, delta, and ending frequencies and higher beginning frequency, producing longer whistles, and showing higher mean number of inflection points compared to dolphins from Gandoca-Manzanillo (Fig. 17a-b).

The difference in the Box-Cox transformed adopted frequencies between populations was marginally significant at the 0.05 level $(F=3.88, p=0.049)$. However, when accounting for the effect of population, behavior, boat presence, and their interaction we found that all interactions affect adopted frequencies: population*behavior (ANCOVA F=7.30, p=0.009), population*boat presence (ANCOVA F=7.64, $\mathrm{p}=0.006)$, and behavior*boat presence (ANCOVA F=7.46, $\mathrm{p}<0.0001$ ).

The coefficient of frequency modulation correlated with duration $\left(\mathrm{R}^{2}=0.36\right.$, $\mathrm{p}<0.0001, \mathrm{~F}=101.84, \mathrm{p}<0.0001$, Fig. 18 ), but not with population or their interaction. When considering the effect of population, boat presence, behavior and their interactions on whistle standard parameters we found that behavior had a significant effect on the coefficient of frequency modulation (ANCOVA F=4.93, $\mathrm{p}=0.0081$ ), duration (ANCOVA $\mathrm{F}=4.93, \mathrm{p}=0.0081$ ), delta and minimum frequency $(\mathrm{ANCOVA} F=3.32, \mathrm{p}=0.038, \mathrm{~F}=6.04$, 
$\mathrm{p}=0.003$ respectively), and the interaction between population and behavior on ending frequency (ANCOVA $\mathrm{F}=3.44, \mathrm{p}=0.034)$.

Comparisons between distant populations: Pairwise comparisons between populations indicate that there are significant differences between bottlenose dolphin whistles from Bocas and Gandoca-Manzanillo and other populations studied in Atlantic (see Tables 11 and 12, Fig. 19). The magnitude of the differences in whistle mean values between the adjacent populations of Bocas and Gandoca-Manzanillo are small ( $p>0.05)$. Interestingly the same pattern was found between Bocas and each of the distant populations ( $\mathrm{p}>0.05)$. The magnitude of the differences, in whistle mean values were significantly higher (particularly in maximum and ending frequencies) between Gandoca-Manzanillo and Texas $\left(\chi^{2}=45.91, \mathrm{p}<0.0023, \mathrm{df}=5\right)$, Brazil $\left(\chi^{2}=35.38, \mathrm{p}<0.013, \mathrm{df}=5\right)$, and Argentina $\left(\chi^{2}=40.43, p<0.009, \mathrm{df}=5\right)$.

\section{Factors promoting whistle variation}

Sympatry: We tested the hypotheses that bottlenose dolphins living in sympatry with the Sotalia guianensis will show significantly greater differences in their whistle parameters mean values relative to Sotalia guianensis than will non-sympatric bottlenose dolphins, as predicted by the 'species hypothesis'. The differences in whistle frequency mean values between Tursiops and Sotalia are significantly larger between sympatric and nonsympatric species $\left(\chi^{2}=1593.8 \mathrm{p}<0.0001, \mathrm{df}=5\right)$. However, the trend is opposite as expected by 'species hypothesis'. The differences between S. guianensis and bottlenose 
dolphins from Gandoca-Manzanillo (sympatric) were significantly smaller than those between $S$. guianensis and bottlenose dolphins from Bocas (non-sympatric) (Fig.20).

Behavior: Bocas del Toro dolphins showed significant differences across behavioral states only in minimum frequency $\left(\chi^{2}=16.26, \mathrm{df}=4, \mathrm{p}=0.0027\right)$, ending frequency $\left(\chi^{2}=12.10, \mathrm{df}=4, \mathrm{p}=0.017\right)$, and in adopted frequencies (ANOVA $\left.\mathrm{F}=10.35, \mathrm{p}<0.0001\right)$ Minimum frequency was higher during foraging and resting, and ending frequency was higher during foraging (Fig. 21a). Adopted frequencies were significantly higher during foraging than during traveling and social activities, but not when the animals were milling or resting. No significant differences were found in other whistle parameters.

Gandoca-Manzanillo dolphins whistled with significantly greater modulation during traveling than foraging (ANOVA F=3.73, $\mathrm{p}=0.029$ ). Dolphins emitted whistles with a greater mean number of harmonics $\left(\chi^{2}=10.43, \mathrm{df}=2, \mathrm{p}=0.005\right)$ during social activities, and when engaged in foraging activities they whistled with lower delta frequency than during social and traveling activities $\left(\chi^{2}=6.22, \mathrm{df}=2, \mathrm{p}=0.044\right)$ (Fig. 21b). No significant differences were found between other standard whistle parameters, adopted frequencies and behavioral states.

Site fidelity: Dolphins from different sites within Bocas showed significant differences in frequency whistle parameters. Dolphins recorded from Bocas Torito whistled with lower minimum $\left(\chi^{2}=11.80, \mathrm{p}=0.02, \mathrm{df}=4\right)$ and maximum frequency $\left(\chi^{2}=9.78, \mathrm{p}=0.04, \mathrm{df}=4\right)$, lower ending $\left(\chi^{2}=23.42, \mathrm{p}=0.0001, \mathrm{df}=4\right)$ and peak frequencies $\left(\chi^{2}=12.84, \mathrm{p}=0.012\right.$, $\mathrm{df}=4$ ) compared to the dolphins recorded from other sites (see Fig.22). 
Ambient noise: Overall noise levels differ significantly between Gandoca-Manzanillo and Bocas $\left(\chi^{2}=5.41, p=0.020\right.$, Fig. 23a $)$. Noise levels were significantly different across frequencies within each site. In Bocas noise levels were particularly high at 2, 10, and 14 $\mathrm{kHz}\left(\right.$ Bocas $\left.\chi^{2}=36.11, \mathrm{p}<0.0001, \mathrm{df}=5\right)$. Noise levels were higher at $2 \mathrm{kHz}$ in GandocaManzanillo $\left(\chi^{2}=22.47, p=0.0004, d f=5\right)$. Sites were significantly different only at $2 \mathrm{kHz}$ $\left(\chi^{2}=4.57, \mathrm{p}=0.033, \mathrm{df}=1\right)$. Ambient noise stations at each study site also varied in noise levels (Bocas: $\chi^{2}=35.23, \mathrm{df}=5, \mathrm{p}<0.0001$, Gandoca-Manzanillo: $\chi^{2}=16.14, \mathrm{df}=2$, $\mathrm{p}=0.0003$ ). In Bocas, the stations Drago, Torito, and Almirante Entrance and stations BEG and MID in Gandoca-Manzanillo had the highest noise levels (Fig. 23b-c). It is important to note that we did not assess directly the relationship between noise levels and whistle structure because our ambient noise data were not measured simultaneously with dolphin recording sessions.

Presence/absence of boats: Boat traffic has been found to be significantly higher in Bocas than in Gandoca-Manzanillo (Taubitz 2007). Because traffic is very low in Gandoca-Manzanillo the sampled size of interactions between dolphins and boats was too low for analysis. Therefore, the following results are just for Bocas. In Bocas dolphins in the presence of boats tended to emit whistles with higher maximum frequency $\left(\chi^{2}=5.02\right.$, $\mathrm{p}=0.025, \mathrm{df}=1)$, greater delta frequency $\left(\chi^{2}=6.74, \mathrm{p}=0.0009, \mathrm{df}=1\right)$, longer duration $\left(\chi^{2}=5.14, p=0.023, \mathrm{df}=1\right)$, and higher mean number of inflection points $\left(\chi^{2}=7.30\right.$, $\mathrm{p}=0.007, \mathrm{df}=1$ ) than when only the research boat was present. Adopted frequencies were also higher in the presence of boats (ANOVA $\mathrm{F}=5.08, \mathrm{p}=0.024$ ) and the coefficient of 
frequency modulation was slightly higher (ANOVA F=4.02, $\mathrm{p}=0.046$ ) when only the research boat was present. 


\section{DISCUSSION}

Dolphin whistles are important communicative signals used in a variety of contexts including, mother and calf recognition, formation of male alliances, group cohesion, etc (e.g., Caldwell and Caldwell 1965; Caldwell et al. 1990, Fripp et al. 2005, Herzing 2000, Janik 2000, Tyack 1997, 2000, Watwood et al. 2004). Because of their important role in social interactions, some of the variation in whistles may reasonably be assumed to facilitate transmission efficiency and avoid signal masking. In general animals are believed to produce signals that are adapted to their particular environment (Peters et al. 2007). Recent studies have found evidence that geographical variation in dolphin whistle acoustic structure may be largely due to local environmental conditions (e.g., Wang et al. 1995, Morisaka et al. 2005, Ansmann et al. 2007). However, other factors such as learning, genetic differentiation (Azevedo and Sluys 2005, Rossi-Santos and Podos 2006, Camargo et al. 2007), and zoogeographical relationships (Steiner 1981) may be important as well. This comparative study provides evidence that dolphin whistles are plastic and appear to be shaped by a combination of factors. The contribution of each of these factors to whistle variation may vary in accordance to local biological and abiotic conditions.

Wang et al. (1995) compared several populations of bottlenose dolphins and found that neighboring populations tended to show a smaller magnitude of whistle variation than distant populations. We found evidence of geographical variation in whistle structure between the adjacent populations of bottlenose dolphins from Bocas and GandocaManzanillo. Bocas dolphins tended to produce lower frequency and longer whistles than Gandoca-Manzanillo dolphins. The significant differences in duration are expected, as it has been shown that duration varies the most within species (e.g., Ding et al. 1995, Barzua- 
Duran and Au 2004, Rendell et al. 1999, Whitten and Thomas 2001). However, the differences in frequency are interesting since these are generally more 'important' in interspecific variation (Wang et al. 1995b, Rendell et al. 1999, May-Collado et al. 2007a).

Both populations also differ significantly in almost all standard whistle parameters with populations in north and south Atlantic. However, the magnitude of the differences between Bocas and Gandoca-Manzanillo were not necessarily smaller than their differences with each of the distant populations. In other words, we did not find evidence that differences in whistle structure were greater between far separated compared to closer populations as found by Wang et al. (1995). Dolphins from Bocas and Gandoca-Manzanillo are as different as other populations in the Atlantic. This may suggest that genetic isolation is important and that these adjacent populations are as isolated from each other as they are from more distant populations. An ongoing Photo-Id study (four years) in these two sites has not yet found evidence of mixing between these populations (May-Collado unpublished data). Until, genetic data are available we cannot estimate the contribution of population isolation to the overall differences in whistle structure between these two populations, but it appears to have a significant role.

Other factors that appear to be influencing whistle structure are local ambient and anthropogenic noise levels. Boat traffic is high in Bocas although low frequency $(2 \mathrm{kHz}$ ambient noise levels are higher in Gandoca-Manzanillo. Dolphins have been shown to respond acoustically to environmental noise in a variety of ways including whistle production rate (Van Parijs and Corkeron 2001, Buckstaff 2004), shifts in signal frequency (from low to high see Lesage et al. 1993), and an increase (Foote et al. 2004) or decrease (Buckstaff 2004) in signal duration. When comparing whistles recorded in presence and absence (not counting 
the research boat) of boats, particularly dolphin-watching boats, we found that Bocas dolphins tend to increase slightly their whistle maximum frequency from $15.35 \mathrm{kHz}$ to 16.74 $\mathrm{kHz}$, and duration from 1.05 to $1.30 \mathrm{sec}$, and their whistle modulation (measured as the mean number of inflection points) from 3.28 to 5.13. However, when accounting for site fidelity, dolphins from Bocas Torito (the area with the most intense dolphin-watching activities, up to 15 boats following a single group) had the lowest frequency parameters (except for beginning frequency) and longest whistles. These results contrast those by Buckstaff (2004) where bottlenose dolphin whistles did not change significantly in frequency range or duration.

Engine noise is due to air bubbles that collapse near the blades of the propellers, which is the most significant source of noise above $2 \mathrm{kHz}$ (Evans et al. 1992). Increasing propeller rotation rate also shifts engine noise to higher frequencies (Richardson et al. 1995), which would have greater potential for masking cetacean signals (Bain and Dahlheim 1994) and may explain the general respond of dolphins to increase their maximum frequencies, like common dolphins in the English Channel were vessels are large with more noise at low frequencies compared with the small boats (with more noise at higher frequencies) in Bocas. In Bocas, for those dolphins that are continuously targeted by dolphin-watching boats, perhaps lowering frequency parameters to be below the noise level, may be a more adequate strategy to avoid masking (like the dolphins from Bocas Torito). While Beluga whales and common dolphins whales have been reported to use higher frequencies when ships are in the area (Lesage et al. 2003; Ansmann et al. 2007), other dolphin species like the Indo-Pacific bottlenose dolphin lower its frequency and modulation to overcome masking. Increased occurrence of long whistles to overcome signal interference, has also been reported in the 
calls of three populations of killer whales where whale-watching activities have become intense (Foote et al. 2004). Erber (2002) estimated whale-watching boat engine levels to be 145 to $169 \mathrm{~dB}$ re $1 \mu \mathrm{Pa} @ 1 \mathrm{~m}$, more than sufficient to mask important signals such as the communicative whistles of dolphins (1 to $35 \mathrm{kHz}$ ) (Richardson et al. 1995). In general, dolphins can overcome signal masking or interference by increasing their frequency, amplitude, and duration (Foote et al. 2004). Dolphins from Gandoca-Manzanillo do not experience intense boat traffic, but overall ambient noise levels are relatively high, particularly at $2 \mathrm{kHz}$. High frequency whistles may help to cope with this. This is also supported by the fact that even in the presence of another dolphin species, Sotalia guianensis, bottlenose dolphins from Gandoca-Manzanillo produced high frequency whistles. According to the 'species hypothesis' sympatric species are expected to be more different than when found separately. Despite the overall differences between these two species, the magnitude of these differences in whistle structure between Sotalia and bottlenose dolphins of GandocaManzanillo are smaller than the differences between Sotalia and bottlenose dolphins from Bocas.

Behavior also played a significant role in whistle variation, but its contribution to this variation is not clear. Overall, behavior and its interaction with population, explained the variation observed in several whistle parameters (duration, COFM, delta, minimum, and ending frequencies). In addition, whistle structure varied across behavioral states within each population. We did not find a common pattern between these two populations and their whistle structure in association with the different behavioral activities. In Bocas, frequency parameters (minimum, ending, and adopted frequencies) varied the most across behaviors, while in Gandoca-Manzanillo frequency modulation (measured as 
COFM), and mean number of harmonics were the most variable parameters. Significant variation of whistle duration has been found in other dolphin species associated with behavioral states or context (Whitten and Thomas 2001), but this was not the case for these two populations. Unfortunately, most studies report whistle production rate but not the frequency and temporal parameters for each behavioral state (e.g., Van Parijs and Corkeron 2001, dos Santos et al. 2005, Nowacek 2005). In common dolphins, behavior was considered a small source of variation in whistle frequency and temporal parameters (Whitten and Thomas 2001, Barzua-Duran and Au 2004, Ansmann et al. 2007) but there were no common patterns between these studies.

Dolphins vary geographically in their whistle structures. The factors that influence these sounds may contribute differently according to local conditions. Furthermore, selection for individual plasticity in whistle structure may be key when living in a continuously changing environment. Our study shows that there are many sources that promote variability between and within populations, but dolphins appear to be plastic and respond differently to these factors. 


\section{CONCLUDING REMARKS}

Whistles are key communicative signals in dolphin societies. These sounds show high inter and intra specific variation. Several factors have been proposed to influence this variation. This study finds that in the case of two adjacent populations both population isolation and environmental variables may contribute significantly to variation in both frequency and temporal parameters. Unlike a previous study, we do not find evidence that neighboring bottlenose dolphin populations are significantly more similar than more distant populations. Both adjacent populations live in contrasting habitats in terms of ambient noise and boat traffic and both factors appear to influence differently each population. Behavioral and zoogeographical relationships with other dolphin species may also have some part in the observed variation but at a much smaller scale. 


\section{ACKNOWLEDGMENTS}

We are indebted to Ingi Agnarsson, Mike Heithaus, Maureen Donnelly, Tim Collins, and Zhemin Chen for their suggestions that improved the manuscript. We thank to Rachel Collins, Gabriel Jacome, and all the staff of Bocas del Toro Research Station, STRI. Thanks to Dennis Lucas, Earl Fernando Junier Wade, and whale-watching operators at the Wildlife Refuge of Gandoca-Manzanillo. This study was carry out with permission of the Ministerio de Ambiente y Energia de la República de Costa Rica and the National Park System, Area de Conservacion Talamanca (Permit No. 137-2005 SINAC) and authorization from the IACUC committee at Florida International University. Funding for this project came from Judith Parker Travel Grant, Lener- Gray Fund for Marine Research of the American Museum of Natural History, Cetacean International Society, Project Aware, The Latin American Student Field Research Award by the American Society of Mammalogists, and the Russell E. Train Education Program-WWF to Laura May-Collado. 


\section{CITED LITERATURE}

Acevedo-Guiterrez, A., \& Stienessen, S.C., 2004. Bottlenose dolphins (Tursiops truncatus) increase number of whistles when feeding. Aquatic Mammals 30:357-362.

Ansmann, I. C., Goold, J. C., Evans, P. G. H., Simmonds, M., \& Simon, G. K., 2007. Variation in the whistle characteristics of short-beaked common dolphins, Delphinus delphis, at two locations around the British Isles. Journal of the Marine Biological Association of the United Kingdom, 87, 19-26.

Azevedo, A. F., \& Van Sluys, M., 2005. Whistles of tucuxi dolphins (Sotalia fluviatilis) in Brazil: comparisons among populations. Journal of the Acoustical Society of America, $117,1456-1464$.

Azevedo, A. F., Oliveira, A.M., Rosa L. D., \& Lailson-Brito, J., 2007. Characteristics of whistles from resident bottlenose dolphins (Tursiops truncatus) in southern Brazil. Journal of the Acoustical Society of America, 121, 2978-2983.

Au, W. W. L., 2000. Hearing in whales and dolphins: An overview. In Hearing by whales and dolphins (eds. W. W. L. Au, A. N. Popper and R. E. Fay), pp. 1-42. New York: Springer Press.

Bain, D. E., \& Dahlheim, M. E., 1994. Effects of masking noise on detection thresholds of killer whales. In Marine mammals and the Exxon Valdez (ed. T. R. Loughlin), pp. 243256. Sand Diego: Academic Press.

Barzúa-Durán, M. C. \& Au, W. W. L., 2002. Whistles of Hawaiian spinner dolphins. Journal of the Acoustical Society of America, 112, 3064-3072.

Barzúa-Durán, M. C. \& Au, W. W. L., 2004. Geographic variations in the whistles of spinner dolphins (Stenella longirostris) of the Main Hawaiian Islands. Journal of the Acoustical Society of America, 116, 3757-3769.

Boncoraglio, G., \& Saino, N., 2007. Habitat structure and the evolution of bird song: a meta-analysis of the evidence for the acoustic adaptation hypothesis. Functional Ecology, $21,134-142$.

Brumm, H., 2006. Animal Communication: City birds have changed their tune. Current Biology, 16, R1003-R1004.

Buckstaff, K., 2004. Effects of watercraft noise on the acoustic behavior of bottlenose dolphins, Tursiops truncatus, in Sarasota Bay, Florida. Marine Mammal Science, 20, 709-725. 
Caldwell, M.C., \& Caldwell, D.K., 1965. Individual whistle contours in bottlenose dolphins (Tursiops truncatus). Nature (Lond.), 207, 434-435.

Caldwell, M. C., Caldwell, D. K., \& Tyack, P. L., 1990. Review of the signature-whistle hypothesis for the Atlantic bottlenose dolphin. In The bottlenose dolphin (eds. S. Leatherwood and R. R. Reeves), pp. 199-234. San Diego: Academic Press.

Camargo, F. S., Rollo, M. M. Jr., Glampaoli, V., \& Bellini, C., 2007. Whistle variability in South Atlantic spinner dolphins from the Fernando de Noronha Archipelago off Brazil. Journal of the Acoustical Society of America, 120, 4071-4079.

dos Santos, M. E., Louro, S., Couchinho, M., \& Brito, C. 2005. Whistles of bottlenose dolphins (Tursiops truncatus) in the Sado Estuary, Portugal: Characteristics, Production Rates, and Long-Term Contour Stability. Aquatic Mammals, 31, 453-462.

Erbe, C., 2002. Underwater noise of whale-watching boats and potential effects on killer whales (Orcinus orca) based on an acoustic impact model. Marine Mammal Science, 18, 394-418.

Evans, P. G. H., Canwell, P.J., \& Lewis, E. 1992. An experimental study of the effets of pleasure craft noise upon bottlenose dolphins in Cardigan Bay, West Wales. In European Research on Cetaceans (Evans P.G.H.), 43-46. European Cetacean Society.

Foote, A. D., Osborne, R. W., \& Hoelzel, R. A., 2004. Whale-call response to masking boat noise. Nature, 428, 910.

Fripp D., Owen C., Quintana-Rizzo E., Shapiro A., Buckstaff K., Jankowski K., Wells R., \& Tyack, P., 2005. Bottlenose dolphin (Tursiops truncatus) calves appear to model their signature whistles on the signature whistles of community members. Animal Cognition, 8, 17-26.

Gillam, E. H., \& McCracken, G. F., 2007. Variability in the echolocation of Tadaria brasiliensis: effects of geography and local acoustic environment. Animal Behaviour. In press.

Herzing, D. L., 2000. Acoustics and social behavior of wild dolphins: implications for a sound society. In Hearing by whales and dolphins (eds. W. W. L. Au, A. N. Popper and R. E. Fay), pp. 225-272. New York: Springer Press.

Janik, V. M., 2000. Whistle matching in wild bottlenose dolphins (Tursiops truncatus). Science, 289, 1355-1357.

Janik, V.M., Dehnhardt, G., \& Todt, D., 1994. Signature whistle variations in bottlenosed dolphin, Tursiops truncatus. Behavioral Ecology and Sociobiology, 35,243-248. 
Lemon. R. E., 1966.Geographic variation in the song of cardinals. Canadian Journal of Zoology, 44, 413-428.

Lesage, V., Barette, C., Kingsley, M. C. S. \& Sjare B., 1999. The effect of vessel noise on the vocal behavior of belugas in the St. Lawrence River estuary, Canada. Marine Mammal Science, 15, 65-84.

Lusseau, D. \& Higham, J. E. S., 2004. Managing the impacts of dolphin-based tourism through the definition of critical habitats: the case of bottlenose dolphins (Tursiops spp.) in Doubtful Sound, New Zealand. Tourism Management, 25, 657-659

Marler, P., 1960. Bird songs and mate selection. In Animal sounds and communication, $7^{\text {th }}$ edition (eds. W. E. Lanyon and W. N. Tavolga), pp. 348-367. Washington DC: American Institute of Biological Sciences.

Matthews, J.N., Rendell, L.E., Gordon, J.C.D., \& MacDonald, D.W. 1999. A review of frequency and time parameters of cetacean tonal calls. Bioacoustics, 10, 47-71. 21

May-Collado, L. J. \& Morales-Ramirez A., 2005. Presencia y patrones de comportamiento del delfin manchado costero, Stenella attenuata graffmani (Cetacea:Delphinidae) en el Golfo de Papagayo, Costa Rica. Revista de Biologia Tropical, 53, 265-276.

May-Collado, L. J. \& Wartzok, D., 2007. The freshwater dolphin Inia geoffrensis geoffrensis produces high frequency whistles. Journal of the Acoustical Society of America, 121, 1203-1212.

May-Collado, L. J., Agnarsson, I., \& Wartzok D., 2007. Reexamining the relationship between body size and tonal signals frequency in whales: a comparative phylogenetic approach. Marine Mammal Science.23, 524-552.

May-Collado, L. J., Agnarsson, I., \& Wartzok D., In press. Phylogenetic review of tonal sound production in whales in relation to sociality. BMC Evolutionary Biology.

McGregor, P. K., Peake, T. M., \& Gilbert, G., 2000. Communication, behaviour, and conservation. In Behaviour and Conservation (eds. L. M. Gosling and W. J. Sutherland), pp. 261-285. Cambridge: Cambridge University Press.

McCowan, B., 1995. A new quantitative technique for categorizing whistles using simulated signals and whistles from captive bottlenose dolphins (Delphinidae, Tursiops truncatus). Ethology, 100, 177-193.

McCowan, B. \& Reiss, D., 1995. Whistle contour development in captive-born infant bottlenose dolphins (Tursiops truncatus): role of learning. Journal of Comparative Psychology 109:242-260. 
Morisaka, T., Shinohara, M., Nakahara, F. \& Akamatsu, T., 2005a. Geographic variation in the whistles among three Indo-Pacific bottlenose dolphin Tursiops aduncus populations in Japa. Fisheries Science, 71, 568-576.

Morisaka, T., Shinohara, M., Nakahara, F. \& Akamatsu, T., 2005b. Effects of ambient noise in the whistles of Indo-Pacific bottlenose dolphin Tursiops aduncus populations in Japan. Journal of Mammalogy, 86, 541-546.

Mundinger, P. C., 1982. Microgeographic and macrogeographic variation in the acquired vocalizations of birds. In Acoustic communication in birds: song learning and its consequences, Vol. 2. (eds. D. E. Kroodsma, Miller E. H., and H. Ouellet), pp. 147-208. San Diego: Sand Diego Academic Press.

Nowacek, D. P., 2005. Acoustic ecology of foraging bottlenose dolphins (Tursiops truncatus), habitat-specific use of three sound types. Marine Mammal Science, 21, 587602.

Peters, R. A., Hemmi, J. M., \& Zeil, J., 2007. Signaling against the wind: modifying motion-signal structure in response to increased noise. Current Biology, 17, 1231-1234.

Rendell, L.E., Matthews, J.N., Gill, A., Gordon, J.C.D., \& MacDonald, D.W., 1999. Quantitative analysis of tonal calls from five odontocete species, examining interspecific and intraspecific variation. Journal of Zoology, 249, 403-410.

Richardson, W. J., Greene, C. R. J., Malme, C. I., \& Thomsom, D. H., 1995. Marine Mammals and Noise, New York: Academic.

Rossi-Santos, M. R., \& Podos, J., 2006. Latitudinal variation in whistle structure of the estuarine dolphin Sotalia guianensis. Behaviour, 143, 347-364.

Smolker, R. A,Richards A. F., Connor, R.C., and J. W. Pepper. 1992. Sex differences in patterns of association among Indian Ocean bottlenose dolphins. Behavior 123(1-2): 3869

Sokal R. R., \& Rohlf, F. J., 1995. Biometry. 3rd ed. W. H. Freeman and Company, New York.

Steiner, W. W., 1981. Species-specific differences in pure tonal whistle vocalizations of five western North Atlantic dolphin species. Behavioral Ecology and Sociobiology, 9, 241-246.

Taubitz. E., 2007. Potential effect of whale-watching engine noise on the vocal behavior of bottlenose dolphins (Tursiops truncatus) in Bocas del Toro, Panama and Manzanillo, Costa Rica. Diplomarbeit, Hansestadt Rostock, 62 pp. 
Tyack, P. L., 1997. Development and social functions of signature whistles in bottlenose dolphins Tursiops truncatus. Bioacoustics 8, 21-46

Tyack, P. L., 2000. Functional aspects of cetacean communication. In Cetacean Societies: Field studies of dolphins and whales (eds. J. Mann, R. C. Connor, P. L. Tyack and H. Whitehead), pp. 270-307. Chicago: The University of Chicago Press.

Van Parijs, S. M. \& Corkeron, P. J., 2001. Vocalizations and behaviour of Pacific Humbpaback dolphins, Sousa chinensis. Ethology. 107, 701-716.

Wang, D., Würsig, B., \& Evans, W. E., 1995a. Comparisons of whistles among seven odontocete species. In Sensory Systems of Aquatic Mammals (eds. R. A. Kastelein, J. A. Thomas, and P. E. Nachtigall). pp. 299-323. The Netherlands: De Spill Publishers.

Wang, D., Wursig, B., \& Evans, W. E., 1995b. Whistles of bottlenose dolphins: comparisons among populations". Aquatic Mammalogy, 21, 65-77.

Watwood S. L., Tyack P. L., \& Wells, R. S., 2004. Whistle sharing in paired male bottlenose dolphins, Tursiops truncatus. Behavioral Ecology and Sociobiology, 55, 531543.

Whitten, J. L., \& Thomas, J. A., 2001. Whistle repertoire of Pacific white-sided dolphins (Lagenorhynchus obliquidens) at the John G. Shedd Aquarium. Journal of the Acoustical Society of America, 109, 2391. 
Table 10. Descriptive statistics of whistle acoustic parameters for both dolphin populations (see Fig. 16).

\begin{tabular}{|c|c|c|c|c|c|c|}
\hline ACOUSTICAL PARAMETER & $\operatorname{MEAN} \pm \mathrm{SD}$ & RANGE & C.V. $\%$ & $\operatorname{MEAN} \pm \mathrm{SD}$ & RANGE & C.V. $\%$ \\
\hline & \multicolumn{3}{|c|}{ BOCAS DEL TORO $(\mathrm{n}=128)$} & \multicolumn{3}{|c|}{ WILDLIFE REFUGE OF GANDOCA-MANZANILLO (n=77) } \\
\hline $\begin{array}{l}\text { Minimum Frequency }(\mathrm{kHz}) \\
(p>0.05)\end{array}$ & $5.27 \pm 1.76$ & $1.6-11.9$ & 0.334 & $5.68 \pm 2.24$ & $1.61-10.85$ & 0.393 \\
\hline $\begin{array}{l}\text { Maximum Frequency }(\mathrm{kHz})^{*} \\
(\text { Mann-Whitney } U=12145, p=0.012)\end{array}$ & $15.84 \pm 3.65$ & $8.35-26.54$ & 0.231 & $17.61 \pm 4.93$ & $8.77-28.48$ & 0.280 \\
\hline $\begin{array}{l}\text { Delta Frequency }(\mathrm{kHz})^{*} \\
\text { (Mann-Whitney } U=4003, p=0.025)\end{array}$ & $10.56 \pm 3.75$ & $3.25-20.14$ & 0.355 & $11.94 \pm 4.32$ & $4.21-22.89$ & 0.362 \\
\hline $\begin{array}{l}\text { Beginning Frequency }(\mathrm{kHz})^{*} \\
(\text { Mann-Whitney } U=5984, p=0.010)\end{array}$ & $9.95 \pm 3.78$ & $3.43-19.63$ & 0.380 & $8.43 \pm 3.66$ & $1.61-17.21$ & 0.435 \\
\hline $\begin{array}{l}\text { Ending Frequency }(\mathrm{kHz})^{*} \\
(\text { Mann-Whitney } U=2332.5, p<0.001)\end{array}$ & $8.43 \pm 4.0$ & $1.64-21.38$ & 0.469 & $13.15 \pm 5.57$ & $4.13-27.14$ & 0.424 \\
\hline $\begin{array}{l}\text { Peak }(\mathrm{kHz}) \\
(p>0.05)\end{array}$ & $10.40-3.20$ & $5.27-21.10$ & 0.308 & $10.64 \pm 4.24$ & $4.13-28.32$ & 0.399 \\
\hline $\begin{array}{l}\text { Duration (s)* } \\
\text { (Mann-Whitney } U=6131.5, p=0.003 \text { ) }\end{array}$ & $1.14-0.69$ & $0.061-3.35$ & 0.603 & $0.89 \pm 0.69$ & $0.087-3.40$ & 0.771 \\
\hline $\begin{array}{l}\text { Number of Inflection Points* } \\
\text { (Mann-Whitney } U=5800, p<0.031)\end{array}$ & $3.93 \pm 4.10$ & $0-20$ & 1.04 & $2.64 \pm 3.41$ & $0-19$ & 1.295 \\
\hline $\begin{array}{l}\text { Number of Harmonics } \\
(p>0.05)\end{array}$ & $1.47 \pm 2.05$ & $0-15$ & 1.399 & $2.34 \pm 1.16$ & $0-13$ & 1.157 \\
\hline $\begin{array}{l}\text { COFM } \\
\text { (Mann-Whitney } U=5473, p=0.185 \text { ) }\end{array}$ & $4.96 \pm 4.11$ & $0.32-20.31$ & 0.828 & $4.80 \pm 6.53$ & $0.439-48.725$ & 1.360 \\
\hline
\end{tabular}


Table 11. Bottlenose dolphin standard whistle parameters from this study and with other studied populations in the Atlantic

\begin{tabular}{|c|c|c|c|c|c|c|c|c|}
\hline STUDY & POPULATION & $N$ & $\operatorname{MIN}(K H z)$ & $\operatorname{MAX}(\mathrm{KHZ})$ & $B E G(K H z)$ & $E_{N D}(K H z)$ & DURATION (S) & $\# I P$ \\
\hline This study & $\begin{array}{l}\text { Bocas del Toro, Panama } \\
\text { (CA)* }\end{array}$ & 128 & $5.27 \pm 1.76$ & $15.84 \pm 3.65$ & $9.95 \pm 3.78$ & $8.43 \pm 4.0$ & $1.14-0.69$ & $3.93 \pm 4.10$ \\
\hline This study & $\begin{array}{l}\text { Gandoca-Manzanillo, } \\
\text { Costa Rica (CA)* }\end{array}$ & 77 & $5.68 \pm 2.24$ & $17.61 \pm 4.93$ & $8.43 \pm 3.66$ & $13.15 \pm 5.57$ & $0.89 \pm 0.69$ & $2.64 \pm 3.41$ \\
\hline This study & $\begin{array}{l}\text { 'Southern Central } \\
\text { America' } \\
\text { (both sites together)* }\end{array}$ & 205 & $5.43 \pm 1.96$ & $16.50 \pm 4.26$ & $9.38 \pm 3.80$ & $10.20 \pm 5.15$ & $1.04 \pm 0.69$ & $3.44 \pm 3.90$ \\
\hline Azevedo et al. 2007 & $\begin{array}{l}\text { Patos Lagoon, Brazil } \\
\text { (SA)* }\end{array}$ & 788 & $5.96 \pm 2.15$ & $12.21 \pm 3.20$ & $8.28 \pm 3.11$ & $8.37 \pm 3.7$ & $0.553 \pm 0.394$ & $1.42 \pm 1.85$ \\
\hline Wang et al. 1995 & $\begin{array}{l}\text { Golfo San Jose, } \\
\text { Argentina (SA)* }\end{array}$ & 110 & $5.91 \pm 1.5$ & $13.65 \pm 1.54$ & $9.24 \pm 2.74$ & $6.63 \pm 2.29$ & $1.14 \pm 0.49$ & $1.58 \pm 1.24$ \\
\hline Wang et al. 1995 & Texas, USA (NA)* & 2022 & $5.77 \pm 1.84$ & $11.32 \pm 3.31$ & $8.01 \pm 2.81$ & $8.16 \pm 3.78$ & $0.68 \pm 0.40$ & $2.09 \pm 2.54$ \\
\hline Steiner & $\begin{array}{l}\text { Western North Atlantic } \\
\text { Ocean (NA)** }\end{array}$ & 857 & $7.33 \pm 1.66$ & $16.235 \pm 2.688$ & $11.26 \pm 3.98$ & $10.225 \pm 3.646$ & $1.30 \pm 0.63$ & $2.86 \pm 2.45$ \\
\hline $\begin{array}{l}\mathrm{CA}=\text { Central Atlanctic } \\
\mathrm{SA}=\text { Southern Atlantic } \\
\mathrm{NA}=\text { Northern Atlantic } \\
* \text { Coastal populations } \\
* * \text { Some appear to be o } \\
\Omega \text { significantly highe }\end{array}$ & $\begin{array}{l}\text { ern most area) } \\
\text { c and other two of the recor } \\
\text { ue }\end{array}$ & & 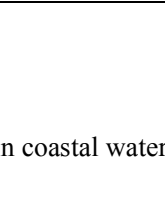 & Caribbean & & & & \\
\hline
\end{tabular}


Table 12. Pairwise comparison of whistle standard parameters between populations, significant values a the $p$-value level of $p<0.05$

\begin{tabular}{|c|c|c|c|c|c|c|}
\hline COMPARISIONS & $\operatorname{MIN}(K H Z)$ & MAX $(K H Z)$ & $B E G(K H Z)$ & END $(K H Z)$ & DURATION $(S)$ & $\# I P$ \\
\hline Bocas vs Brazil & $\mathrm{t}=3.44, \mathrm{P}<0.0006$ & $\mathrm{t}=42.93, \mathrm{P}<0.0001$ & $\mathrm{t}=5.40, \mathrm{P}<0.0001$ & $\mathrm{P}>0.05$ & $\mathrm{t}=13.78, \mathrm{P}<0.0001$ & $\mathrm{t}=11.46, \mathrm{P}<0.0001$ \\
\hline Bocas vs. Argentina & $\mathrm{t}=3.26, \mathrm{P}<0.001$ & $\mathrm{t}=5.86, \mathrm{P}<0.0001$ & $\mathrm{P}<0.005$ & $\mathrm{t}=4.17, \mathrm{P}<0.0001$ & $\mathrm{P}>0.05$ & $\mathrm{t}=5.78, \mathrm{P}<0.0001$ \\
\hline Bocas vs Texas & $\mathrm{t}=2.99, \mathrm{P}<0.003$ & $\mathrm{t}=14.88, \mathrm{P}<0.0001$ & $\mathrm{t}=7.35, \mathrm{P}<0.0001$ & $\mathrm{P}>0.05$ & $\mathrm{t}=11.94, \mathrm{P}<0.0001$ & $\mathrm{t}=7.59, \mathrm{P}<0.0001$ \\
\hline Bocas vs Western NA & $\mathrm{t}=13.10, \mathrm{P}<0.0001$ & $\mathrm{P}>0.05$ & $\mathrm{t}=3.48, \mathrm{P}<0.0005$ & $\mathrm{t}=5.13, \mathrm{P}<0.0001$ & $\mathrm{t}=2.64, \mathrm{P}<0.0080$ & $\mathrm{t}=4.10, \mathrm{P}<0.0001$ \\
\hline Gandoca-Manzanillo vs Brazil & $\mathrm{P}>0.05$ & $\mathrm{t}=13.35, \mathrm{P}<0.0001$ & $\mathrm{P}>0.05$ & $\mathrm{t}=11.01, \mathrm{P}<0.0001$ & $\mathrm{t}=6.59, \mathrm{P}<0.0001$ & $\mathrm{t}=5.10, \mathrm{P}<0.0001$ \\
\hline Gandoca-Manzanillo vs. Argentina & $\mathrm{P}>0.05$ & $\mathrm{t}=7.89, \mathrm{P}<0.0001$ & $\mathrm{P}>0.05$ & $\mathrm{t}=11.62, \mathrm{P}<0.0001$ & $\mathrm{t}=2.89, \mathrm{P}<0.0043$ & $\mathrm{t}=3.00, \mathrm{P}<0.0031$ \\
\hline Gandoca-Manzanillo vs Texas & $\mathrm{P}>0.05$ & $\mathrm{t}=16.02, \mathrm{P}<0.0001$ & $\mathrm{P}>0.05$ & $\mathrm{t}=11.92, \mathrm{P}<0.0001$ & $\mathrm{t}=4.36, \mathrm{P}<0.0001$ & $\mathrm{P}>0.05$ \\
\hline Gandoca-Manzanillo vs Western NA & $\mathrm{P}>0.05$ & $\mathrm{t}=3.94, \mathrm{P}<0.0001$ & $\mathrm{t}=6.01, \mathrm{P}<0.0001$ & $\mathrm{t}=7.17, \mathrm{P}<0.0001$ & $\mathrm{t}=5.43, \mathrm{P}<0.0001$ & $\mathrm{P}>0.05$ \\
\hline Bocas vs Gandoca-Manzanillo & See Table 10 & & & & & \\
\hline Southern Central America vs Brazil & $\mathrm{t}=3.20, \mathrm{P}<0.0014$ & $\mathrm{t}=15.88, \mathrm{P}<0.0001$ & $\mathrm{t}=4.30, \mathrm{P}<0.0001$ & $\mathrm{t}=5.78, \mathrm{P}<0.0001$ & $\mathrm{t}=14.17, \mathrm{P}<0.0001$ & $\mathrm{t}=10.65, \mathrm{P}<0.0001$ \\
\hline Southern Central America vs. Argentina & $\mathrm{t}=2.24, \mathrm{P}<0.0258$ & $\mathrm{t}=6.78, \mathrm{P}<0.0001$ & $\mathrm{P}>0.05$ & $\mathrm{t}=6.91, \mathrm{P}<0.0001$ & $\mathrm{P}>0.05$ & $\mathrm{t}=4.86, \mathrm{P}<0.0001$ \\
\hline Southern Central America vs Texas & $\mathrm{t}=2.51, \mathrm{P}<0.012$ & $\mathrm{t}=20.74, \mathrm{P}<0.0001$ & $\mathrm{t}=6.41, \mathrm{P}<0.0001$ & $\mathrm{t}=7.08, \mathrm{P}<0.0001$ & $\mathrm{t}=11.30, \mathrm{P}<0.0001$ & $\mathrm{t}=6.84, \mathrm{P}<0.0001$ \\
\hline Southern Central America vs Western NA & $\mathrm{t}=14.22, \mathrm{P}<0.0001$ & $\mathrm{P}>0.05$ & $\mathrm{t}=6.10, \mathrm{P}<0.0001$ & $\mathrm{P}<0.05$ & $\mathrm{t}=5.13, \mathrm{P}<0.0001$ & $\mathrm{t}=2.61, \mathrm{P}<0.0092$ \\
\hline Argentina vs Brazil & $\mathrm{P}>0.05$ & $\mathrm{t}=4.64, \mathrm{P}<0.0001$ & $\mathrm{t}=3.11, \mathrm{P}<0.002$ & $\mathrm{t}=4.80, \mathrm{P}<0.0001$ & $\mathrm{t}=14.17, \mathrm{P}<0.0001$ & $\mathrm{P}>0.05$ \\
\hline Texas vs. Brazil & $\mathrm{t}=2.34, \mathrm{P}<0.019$ & $\mathrm{t}=6.46, \mathrm{P}<0.0001$ & $\mathrm{t}=2.14, \mathrm{P}<0.032$ & $\mathrm{P}>0.05$ & $\mathrm{t}=7.59, \mathrm{P}<0.0001$ & $\mathrm{t}=6.74, \mathrm{P}<0.0001$ \\
\hline Western NA vs. Brazil & $\mathrm{t}=14.56, \mathrm{P}<0.0001$ & $\mathrm{t}=27.70, \mathrm{P}<0.0001$ & $\mathrm{P}>0.05$ & $\mathrm{t}=10.24, \mathrm{P}<0.0001$ & $\mathrm{t}=28.05, \mathrm{P}<0.0001$ & $\mathrm{t}=13.10, \mathrm{P}<0.0001$ \\
\hline Argentina vs/Texas & $\mathrm{P}>0.05$ & $\mathrm{t}=7.33, \mathrm{P}<0.0001$ & $\mathrm{t}=3.36, \mathrm{P}<0.0008$ & $\mathrm{t}=4.20, \mathrm{P}<0.0001$ & $\mathrm{t}=11.60, \mathrm{P}<0.0001$ & $\mathrm{t}=2.10, \mathrm{P}<0.0358$ \\
\hline Argentina vs Western NA & $\mathrm{t}=8.55, \mathrm{P}<0.0001$ & $\mathrm{t}=9.90, \mathrm{P}<0.0001$ & $\mathrm{t}=5.15, \mathrm{P}<0.0001$ & $\mathrm{t}=10.10, \mathrm{P}<0.0001$ & $\mathrm{t}=2.55, \mathrm{P}<0.0109$ & $\mathrm{t}=7.18, \mathrm{P}<0.0001$ \\
\hline Texas vs Western NA & $\mathrm{t}=4.03, \mathrm{P}<0.0001$ & $\mathrm{t}=38.45, \mathrm{P}<0.0001$ & $\mathrm{P}>0.05$ & $\mathrm{t}=13.54, \mathrm{P}<0.0001$ & $\mathrm{t}=30.70, \mathrm{P}<0.0001$ & $\mathrm{P}<0.0001$ \\
\hline
\end{tabular}


FIG. 16

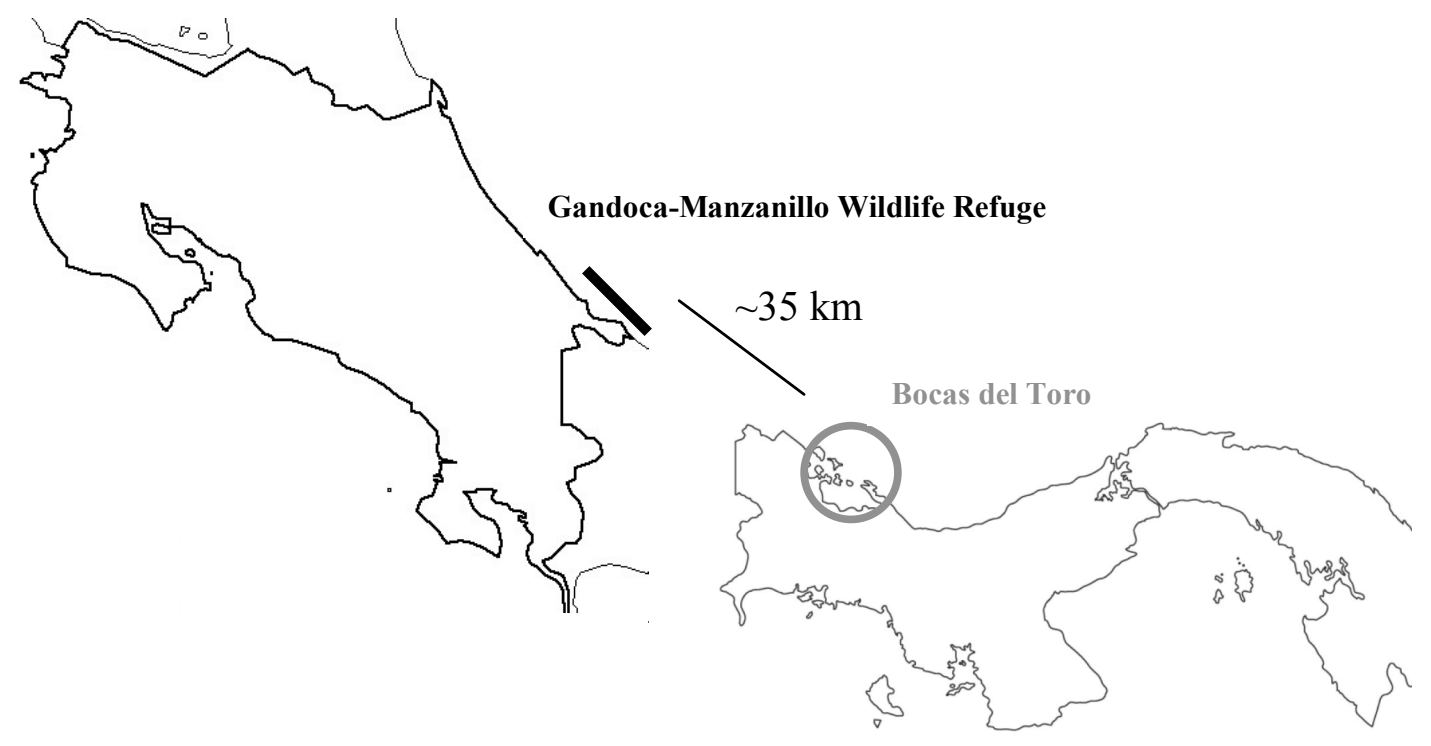


FIG. 17
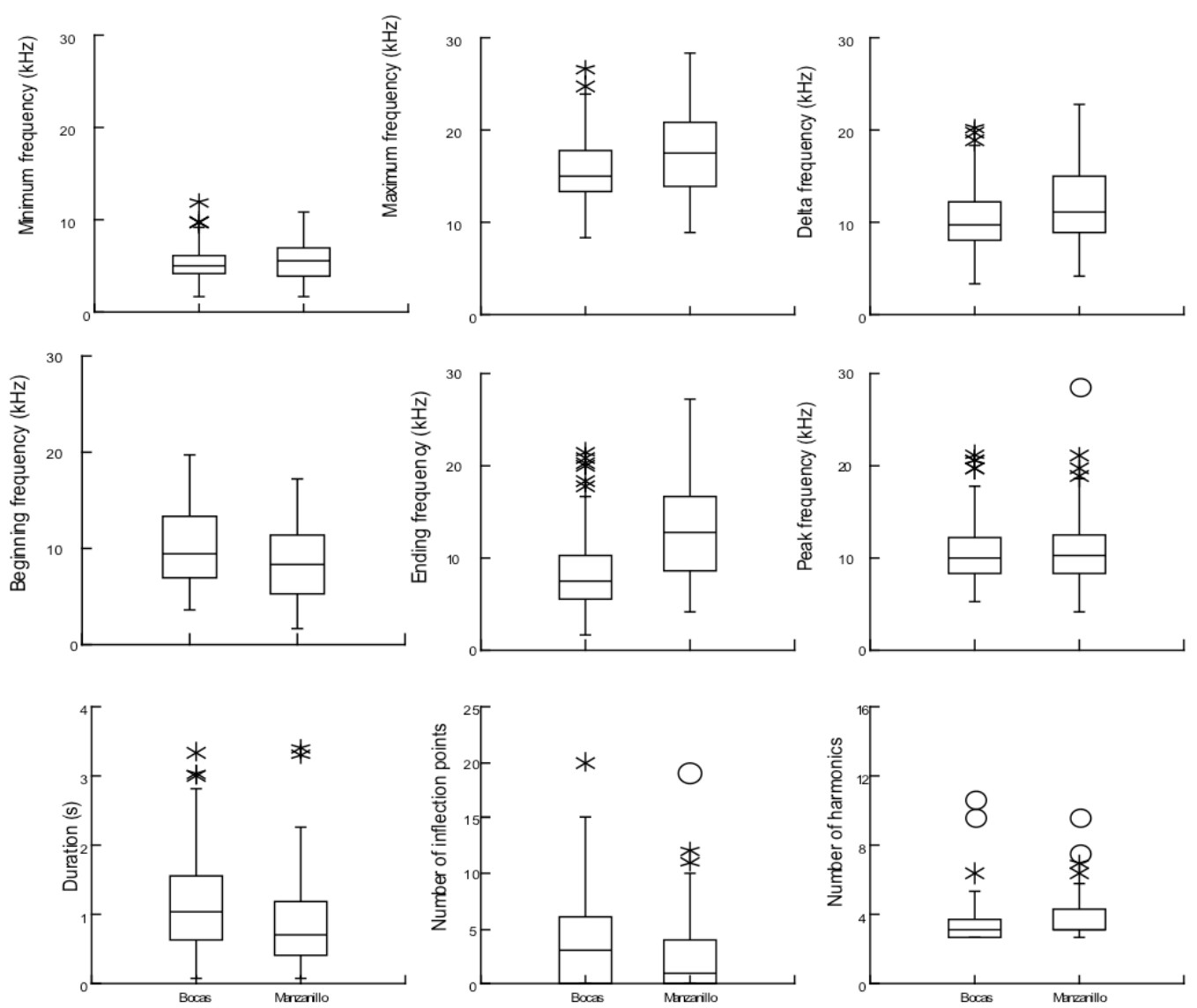
FIG. 18

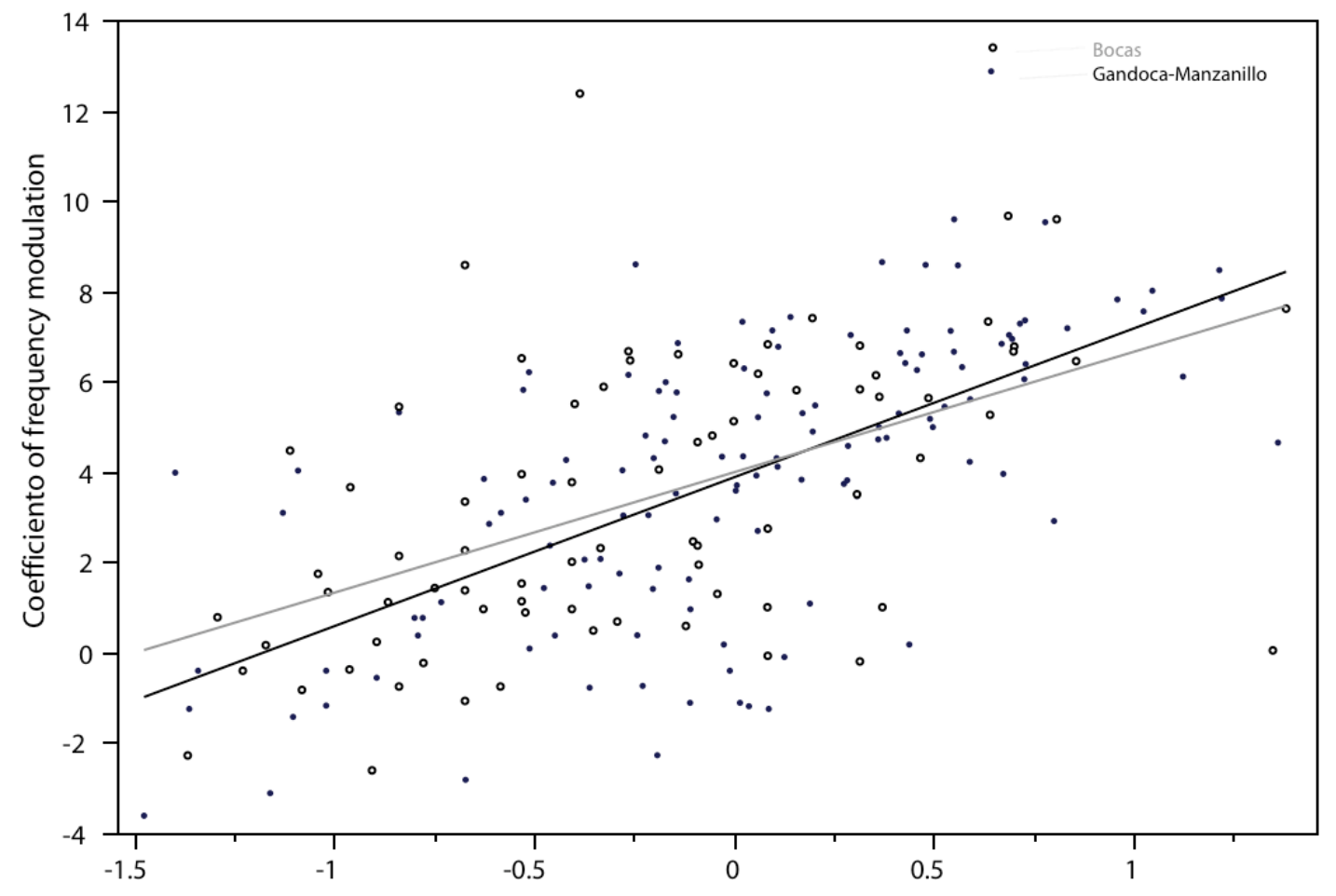

Duration (s) 
FIG. 19

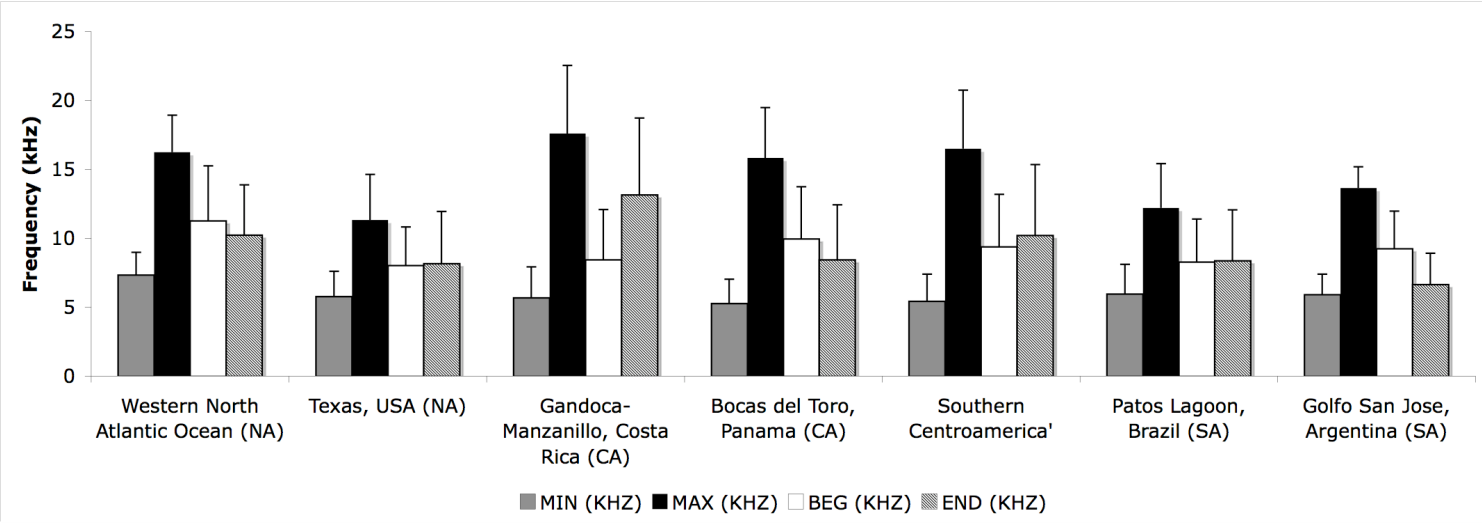

a.

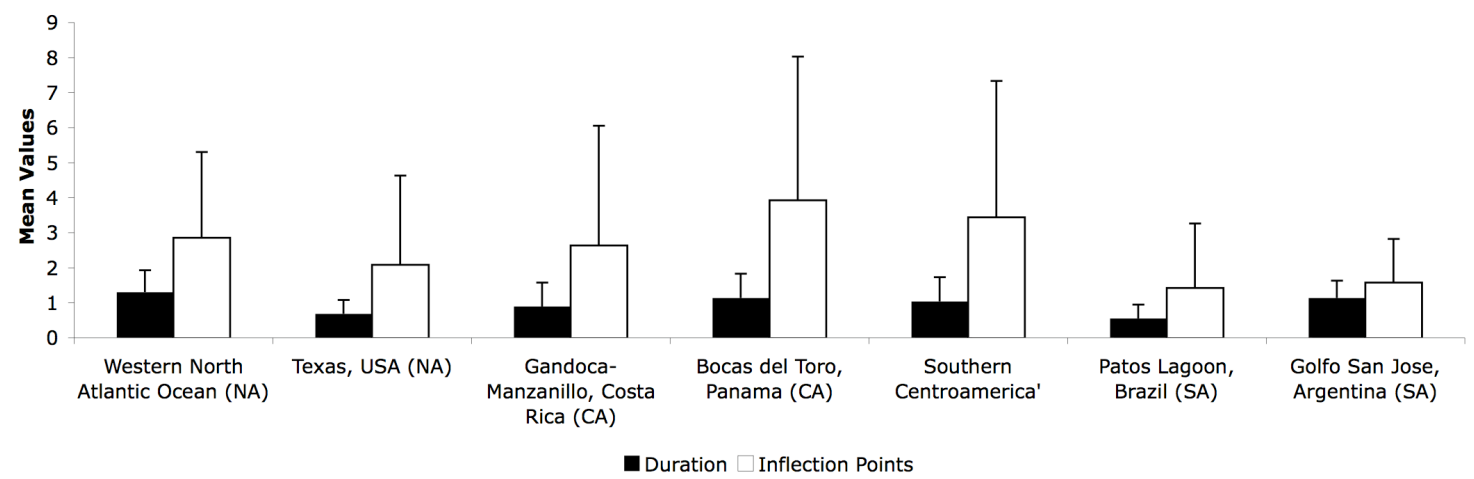

b. 
FIG. 20

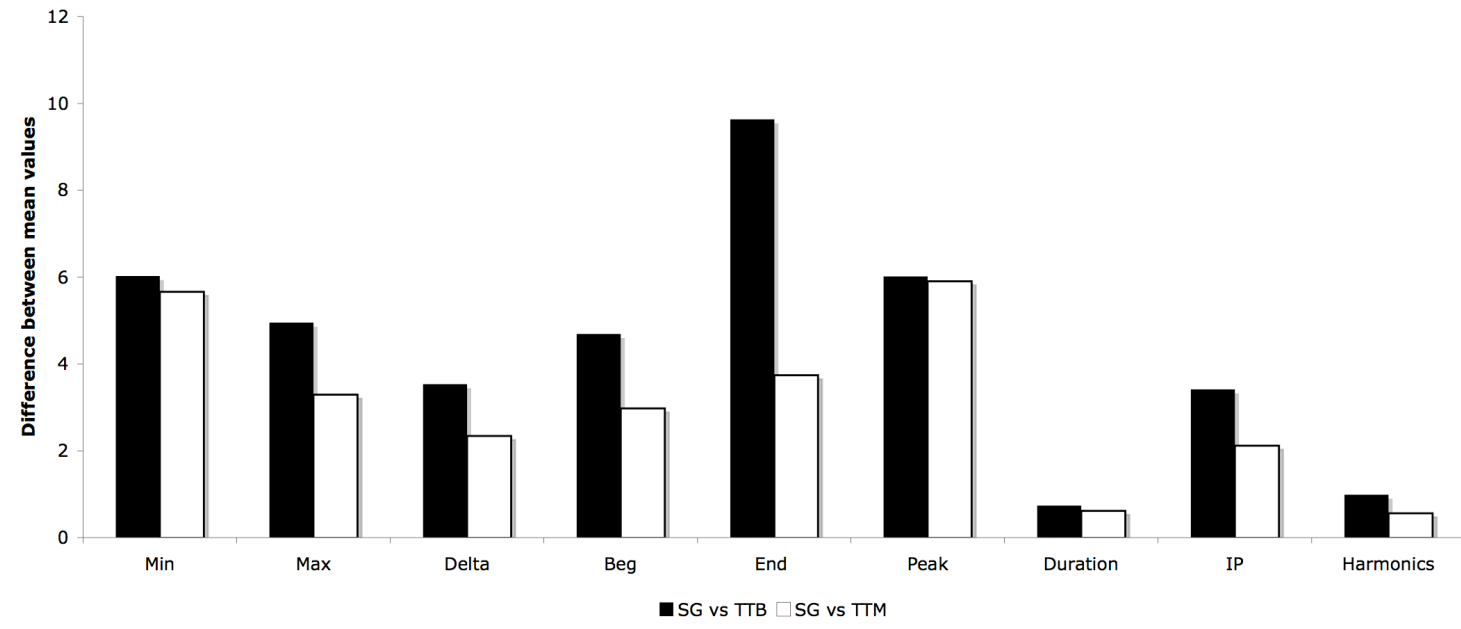


FIG. 21
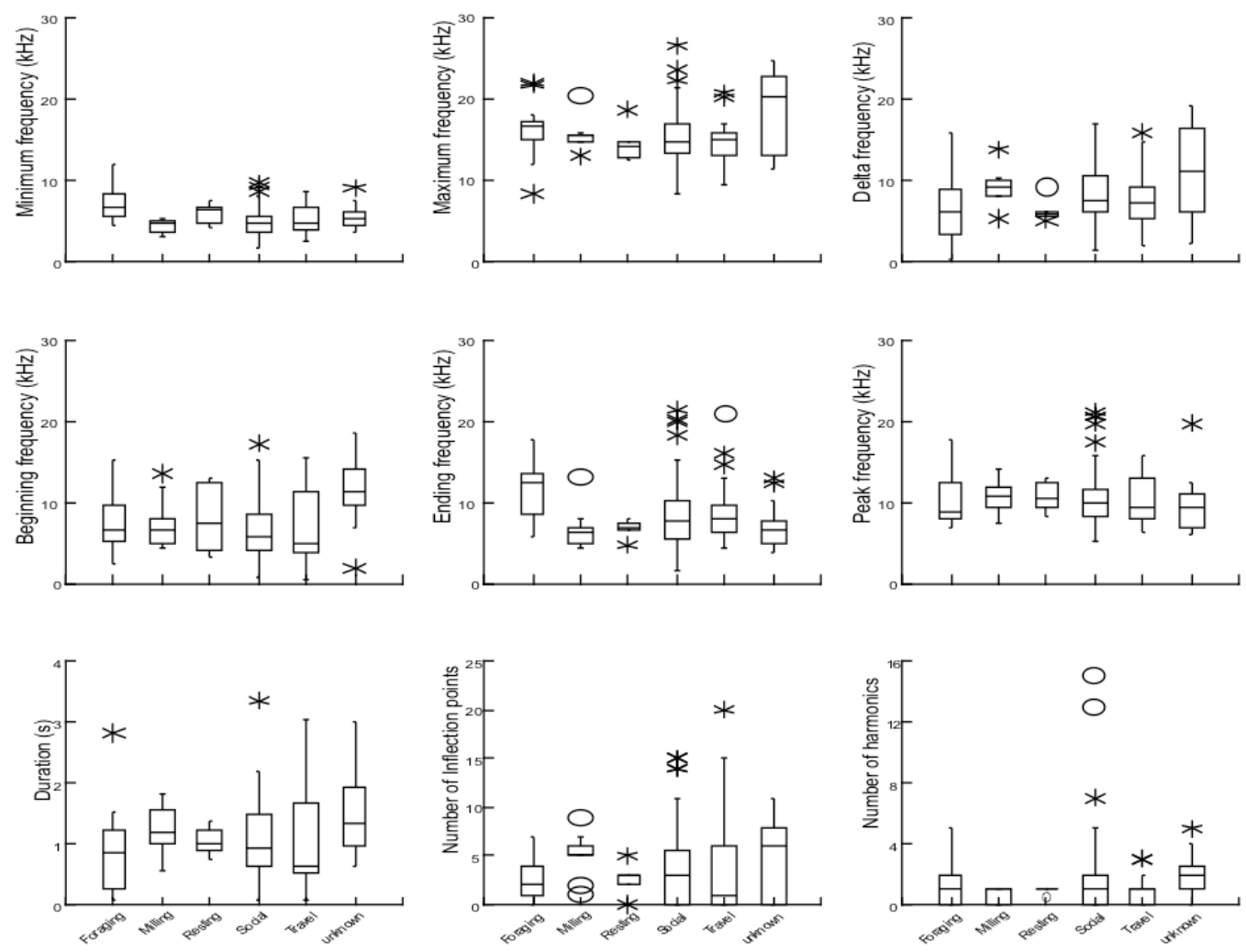

A. Bocas del Toro
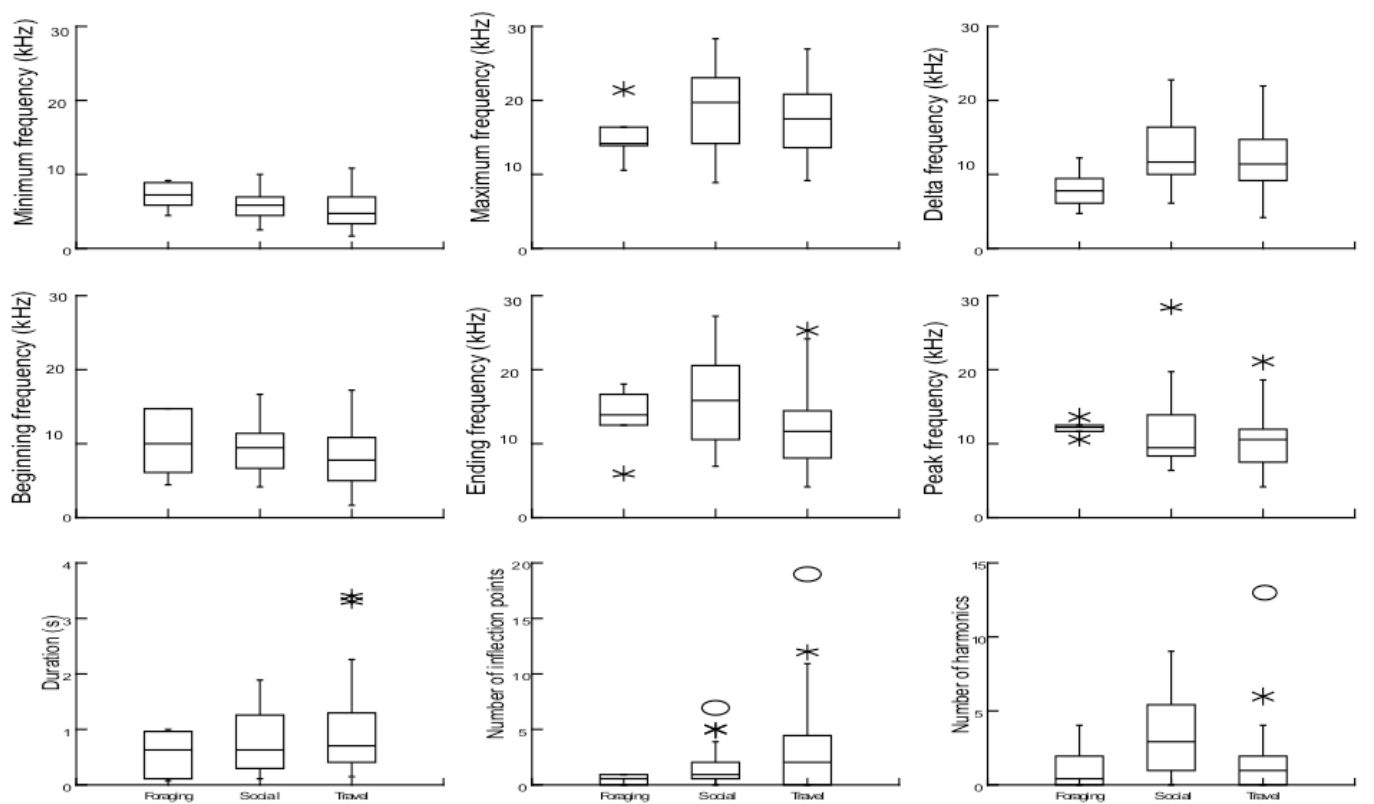

B. Gandoca-Manzanillo 
FIG. 22
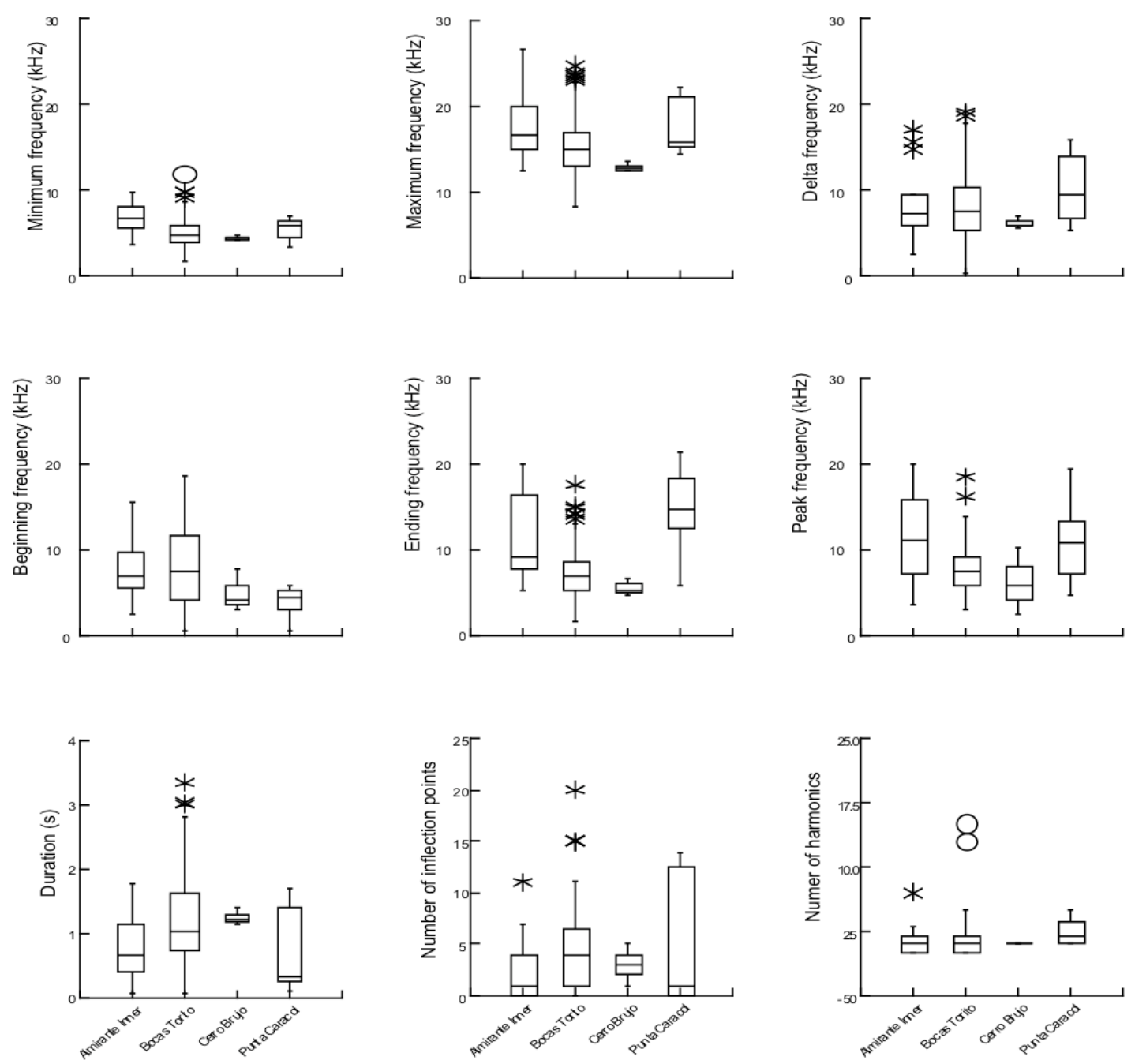
FIG. 23

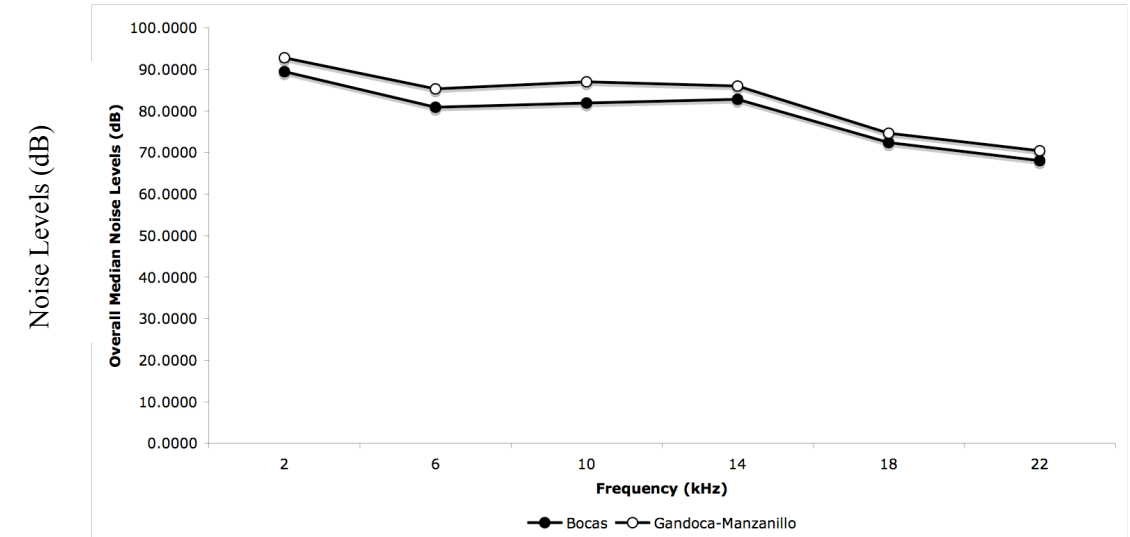

a. Overall median values by study site

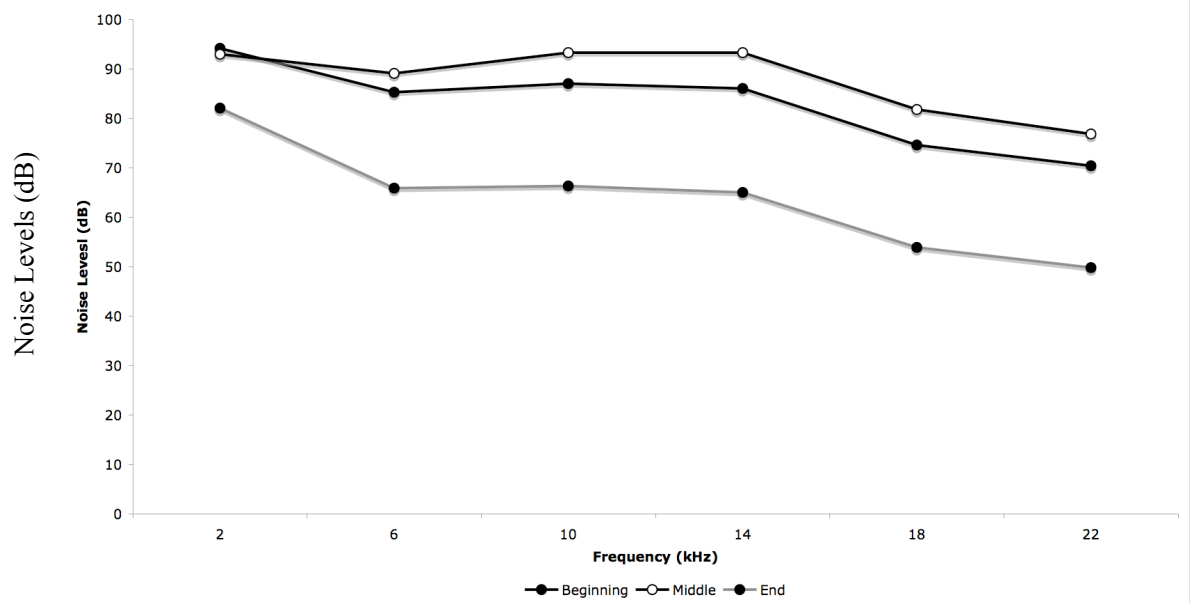

b. Median values for each ambient noise station in Wildlife Refuge of GandocaManzanillo.

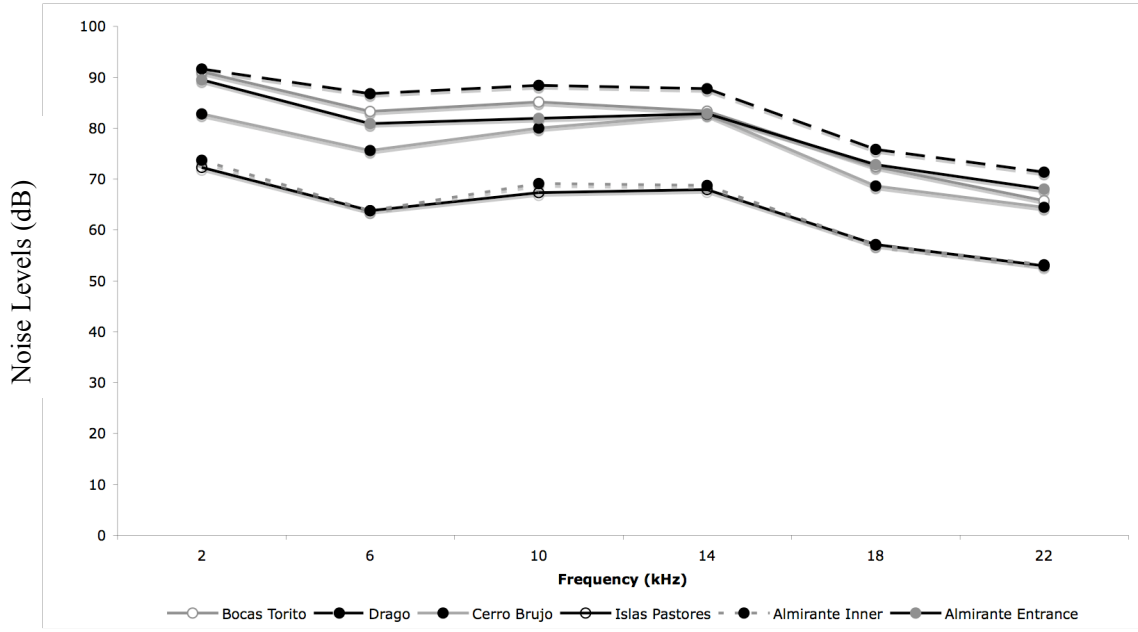

c. Median values for each ambient noise station in Bocas del Toro 


\section{CHAPTER VII}

\section{CONCLUSIONS AND FUTURE RESEARCH DIRECTIONS}

- A detailed species-level cetacean phylogeny was reconstructed to test several evolutionary hypotheses regarding tonal sound evolution. This phylogenetic hypothesis is concordant with well establish benchmark clades previously supported by morphological and mitochondrial and nuclear DNA and thus seems appropriate for hypotheses testing. Both exhaustive taxon sampling and a Bayesian approach for analysis seem to have contributed to phylogenetic accuracy as judged by recovery of benchmark clades. Still, some key clades, most notably ziphiids, remain poorly resolved due to lack of data, and the phylogenetic placement of some lineages is still controversial, e.g. the river dolphin Platanista. My future research directions in this field aims to produce a comprehensive 'total evidence' Cetacean phylogeny by combining multiple datasets that are already available (morphology, nuclear DNA, mitochondrial DNA, SINE). We also aim to continue testing the utility of cyt-b for rapid, but reliable, estimates of phylogenies for other mammal groups, including Cetartiodactyla (Agnarsson and May-Collado in prep.).

- The freshwater dolphin Inia geoffrensis (Boto), emits tonal sounds that, although relatively short, closely resemble in several acoustical parameters the 'whistles' a category of sounds applied by some authors exclusively to social dolphin tonal sounds. Despite the general assumption that river dolphins are solitary, very little is known about their social structure. In some parts of their distribution, at least, they can be found in groups. However, the relationships between group members, 
or the stability of these groups is unknown. Based on our behavioral observations and the distance at which their tonal signals can be detected in their environment, it is possible that at least the study population of the Boto in Ecuador use these tonal for social communication. However, instead of promoting social encounter, we suggest they may be used to maintain distance between individuals or groups. Finally, our results suggest remarkable whistle variation between botos from Ecuador and other populations (Colombia, Peru, and Brazil). Although, this may be largely a product of limitations of recording systems used by previous studies, it will be important to revisit these populations and obtain recordings using a broadband system as the one used in this dissertation. If the differences in whistle structure turn out not simply to be a product of recording limitations, they may represent one of the most remarkable geographical variation ever reported in toothed whales.

- Tonal sound acoustical structure varies across species particularly in frequency parameters. We tested two hypotheses that are believed to shape these frequency parameters through their evolutionary history: body size and sociality. The results of this dissertation find evidence that Cetacean body size has constrained the evolution of tonal sounds minimum frequency (although only a portion of the variation can be explained by body size). This suggests (1) that in the evolutionary history of whales there has been a selection for low frequency sounds, which e.g. enable communication over long distances, and (2) that the degree to which whales have been able to respond to this selection through evolutionary history has been, at least in some cases, constrained by body size. 
However, there is no evidence, that body size has constrained the evolution of any other frequency parameter and alternative hypotheses are required to explain their evolution.

- We find no evidence supporting the 'dolphin hypotheses' stating that 'whistles' evolved within Delphinidae in a social context. Whistles arose earlier in the evolutionary history of whales than hitherto appreciated, however, the evolutionary history of sociality in Cetaceans is complex and several alternative hypotheses are discussed in this dissertation. Nonetheless, sociality does seem to explain some of the variation observed in minimum frequency. Cetacean species that live in large groups tend to produce whistles with higher minimum frequency. In these group-living species, members are generally relatively close to each other so that sounds need not be transmitted over long distances. Furthermore, the evolution of tonal sound complexity seems to be intertwined with social structure:

(1) increased tonal sound modulation (whistle 'complexity') significantly correlates with group size and social structure and (2) changes in tonal sound complexity were significantly concentrated on social branches. For future directions in the study of evolutionary history of sociality and sound communication in Cetaceans it will be important to obtain data on social structure and tonal sounds for a greater number of cetaceans, and to take into consideration the social structure and tonal sound production of outgroups.

- Studying the intraspecific variation of tonal sounds ('whistles') in bottlenose dolphins also proved to be complex. The observed variation appears to be product of a combination of factors that may influence differently each of the acoustical 
parameters in a whistle. However, in the study populations, it appears that isolation and local adaptation to the acoustic characteristics of their habitat are important factors promoting intraspecific variation. Future, studies should assess the genetic isolation between these two populations, and directly measure the relationship between ambient noise levels and whistle frequency and duration by obtaining ambient noise levels from the same recordings from which whistles are extracted. 
APPENDIXES 


\section{APPENDIX I}

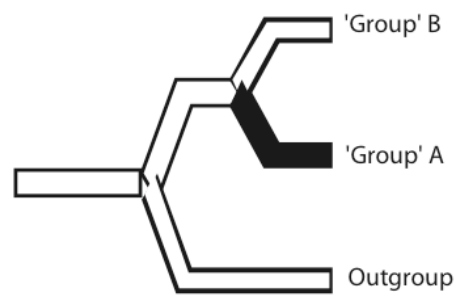

a. Optimization of Snorts

Present

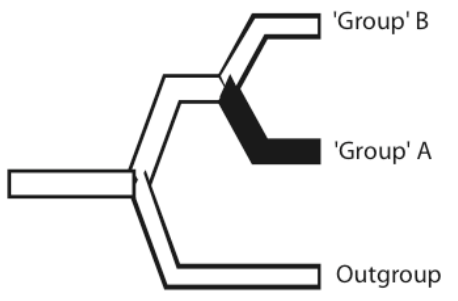

b. Optimization of frequency

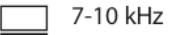

$12-15 \mathrm{kHz}$

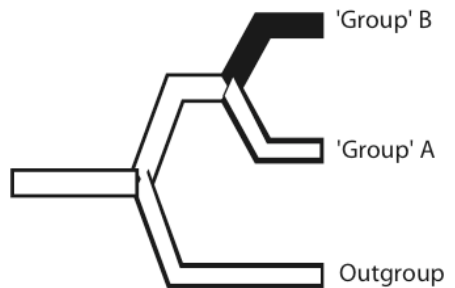

c. Optimization of inflection points

$\square 2$ inflection points

Whistles as a unit for evolutionary analyses. As noted above there are several reasons why using conglomerate concepts like 'whistles' as units of study can hinder progress in the understanding of sound evolution. Apart from being rather arbitrarily defined, and hence differently by different authors, 'whistles' represent a set of characters that may vary independently and may each have different phylogenetic distributions. As a thought experiment let us think of an example where sound production is being compared in two sister lineages. Let us assume that some authors are interested in the evolutionary origin of tonal sounds called 'snorts', and that snorts are defined as narrowband, frequency modulated sounds, with a contour containing at least two inflection points and frequency above $10 \mathrm{kHz}$. In group A it is noted that sounds are narrowband, frequency modulated, with three inflection points and frequency ranging from $12-15 \mathrm{kHz}$. In group B sounds are narrowband, frequency modulated, with a contour of two inflection points and frequency ranging from $7-9 \mathrm{kHz}$. Under a 'broad concept' analysis we would therefore conclude that 'snorts' were present in A, but absent in B, and might conclude that snorts originated in the common ancestor of A (diagram a). However, this belies both the similarities and differences that exist in sound production in the two groups. It denies homology of frequency modulation, contours etc, and even suggests that tonal sounds evolved independently in each group (as 'snorts' are 'different' tonal sounds from nonsnorts). Under a 'component' analysis (diagrams b and c), traits like frequency modulation and band width would be scored as identical in the two groups - their similarity would be taken as evidence of common ancestry, i.e. homology. Instead of 'snorts' originating in A, we would more simply explain the differences between the two groups in terms of frequency, and if e.g., the outgroups shared the lower frequency (indicated by white branches) of B we would conclude that a switch to higher frequency (indicated by black branches) occurred in the common ancestor of A (diagram b). In other words, we would learn that the difference between what people call 'snorts' and what they don't call snorts may simply be a matter of sound frequency. In this latter case there is no indication of tonal sound production being non-homologous in A and B, and in fact they share most characteristics of the tonal sounds. Additionally we would learn (diagram c) that inflection points increased from two (white branches) to three (dark branches) in the lineage leading to B (supposing the condition in A was shared with the outgroups). This is information that the concept of 'snorts' obscured. By a component analysis we learn a 
lot more than by a concept analysis. If we now were interested in the association of sounds and sociality, and group A was social and group B (and outgroups) not, it might be claimed that 'snorts' and 'sociality' are associated and evolved in concert (following diagram a). However, a much more precise and informative conclusion would be that sociality and sound frequency (diagram b) might be related. Hence instead of explaining the social context of 'snorts' we would do well to examine how sound frequency might play an important role in social communication etc. We believe that 'whistles' are no better justified as a unit for evolutionary analysis than 'snorts' in the example above. We do use them in an attempt to test the dolphin hypothesis, but then we opt for a component approach for most of our analyses. 


\section{APPENDIX 2}

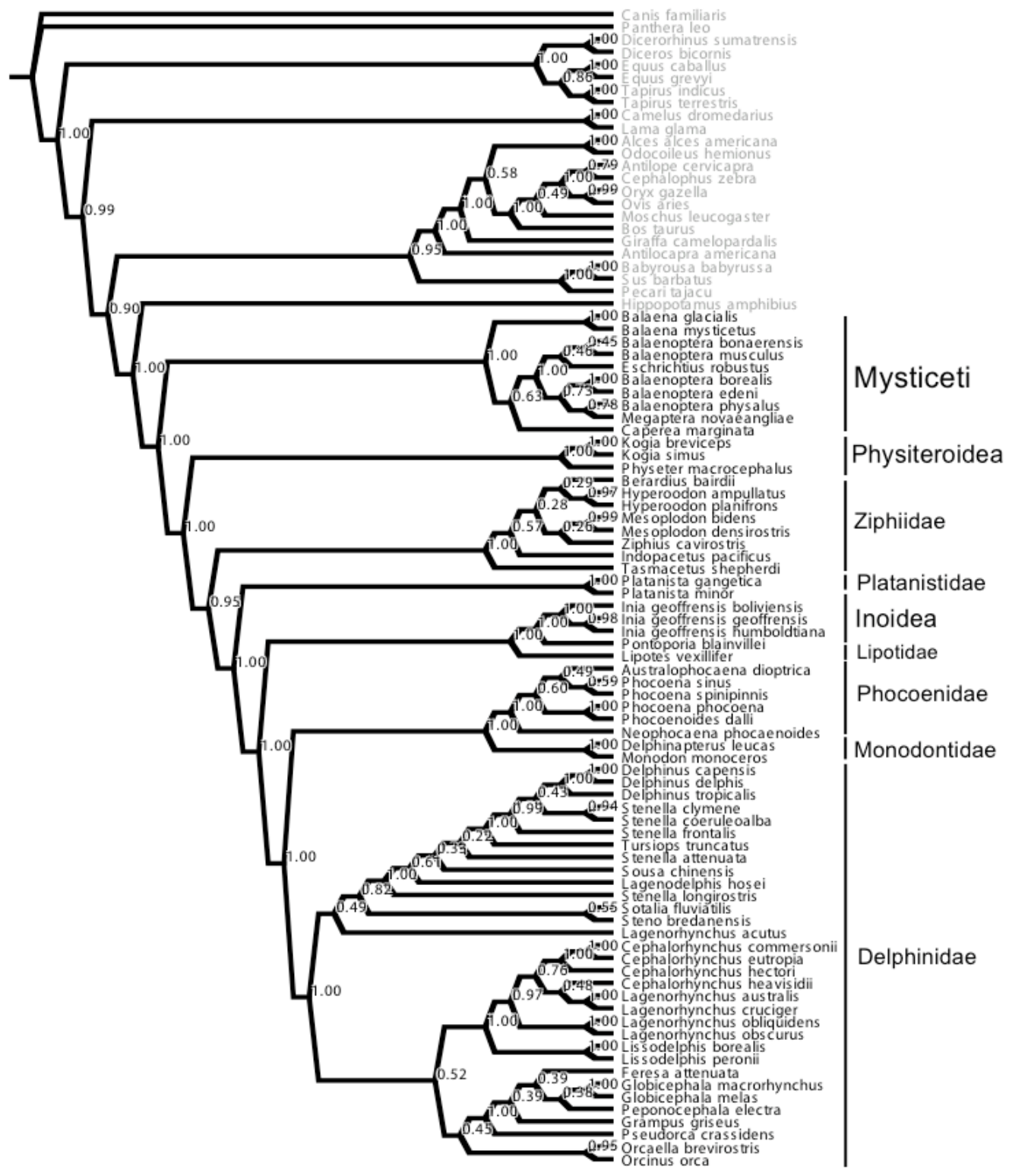

A cetacean phylogeny consistent with Arnason (2004). A majority rule consensus of all post-burnin trees from May-Collado et al. (2007) filtered to be congruent with the mitogenomic phylogeny of Arnason (2004). Numbers on nodes represent posterior probabilities. 


\section{APPENDIX 3}

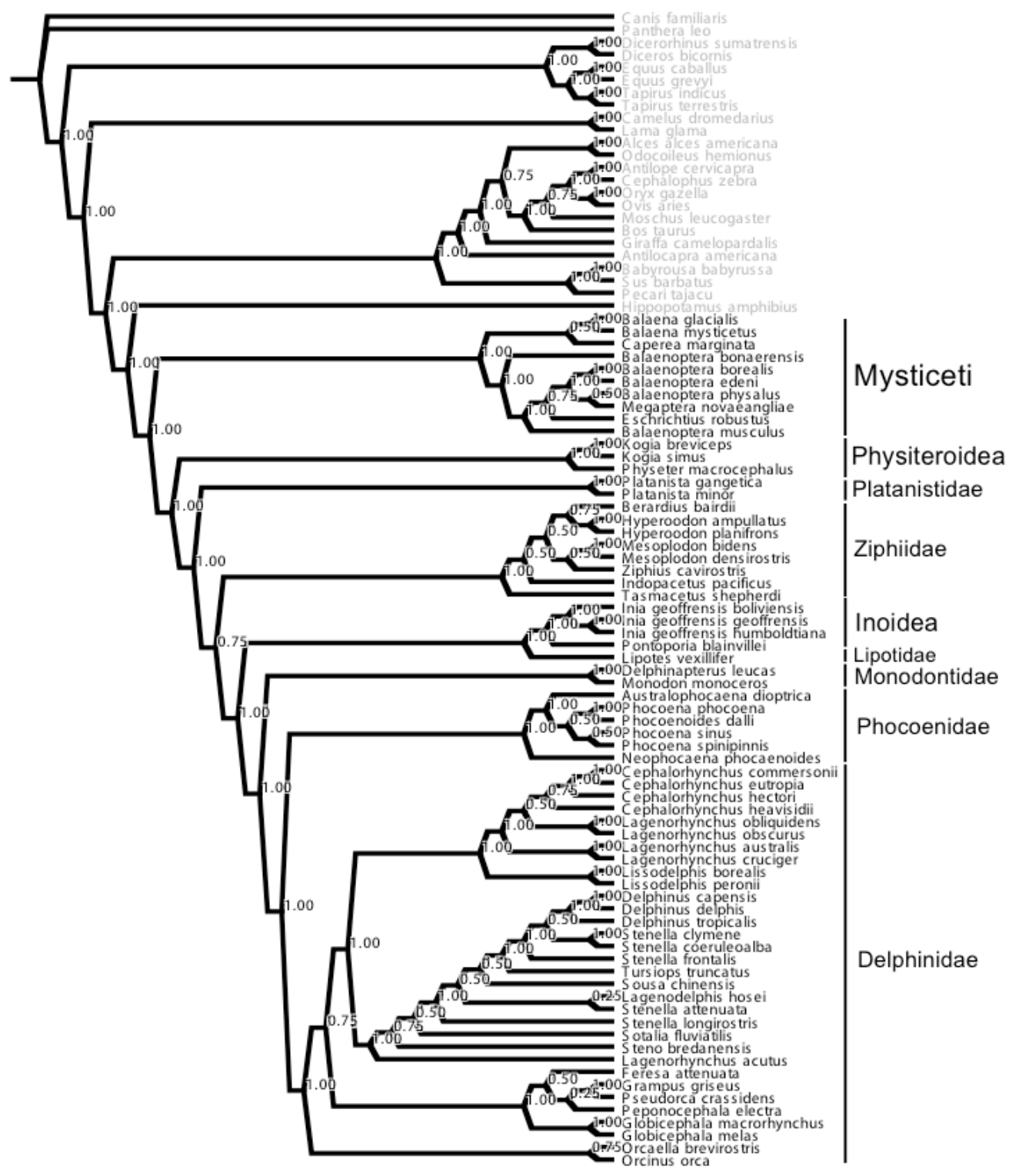

A cetacean phylogeny consistent with Messenger and McGuire (1998). A majority rule consensus of all post-burnin trees from May-Collado et al. (2007) filtered to be congruent with the combined morphological and molecular phylogeny of Messenger and McGuire (1998). Numbers on nodes represent posterior probabilities. 


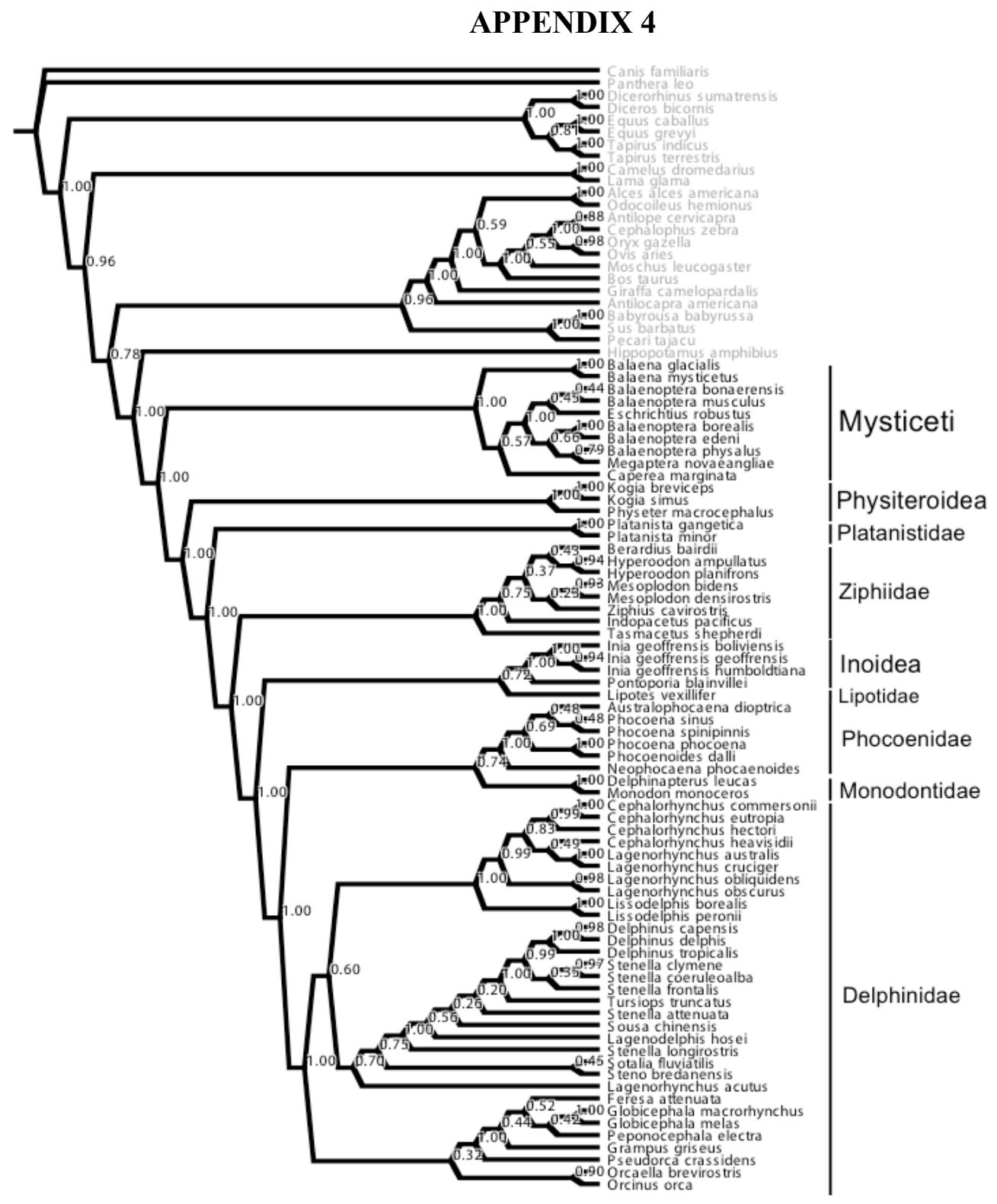

A cetacean phylogeny consistent with Nikaido et al. (2001). A majority rule consensus of all post-burnin trees from May-Collado et al. (2007) filtered to be congruent with the SINE phylogeny of Nikaido et al. (2001). Numbers on nodes represent posterior probabilities. 
APPENDIX 5. Cetacean social structure and group size. This table reviews published data on cetacean social structure and group size. Numbers in parenthesis correspond to state assigned to each characters as described in Table 1 (bold numbers represent the most common state reported for a particular species).

\begin{tabular}{|c|c|c|c|c|c|c|c|}
\hline SPECIES & \multirow{2}{*}{$\begin{array}{l}\text { SOCIALITY 2- } \\
\text { STATES } \\
\text { CHARACTER }\end{array}$} & \multirow{2}{*}{$\begin{array}{l}\text { SOCIALITY } \\
\text { 4-STATES } \\
\text { CHARACTER }\end{array}$} & \multirow{2}{*}{$\begin{array}{l}\text { GROUP } \\
\text { MEAN } \\
\text { SIZE }\end{array}$} & \multicolumn{3}{|c|}{ SOCIALITY COMPONENTS } & \multirow[b]{2}{*}{ REFERENCES } \\
\hline $\begin{array}{l}\text { BALEEN WHALES } \\
\text { (MYSTICETI) } \\
\text { BALAENIDAE } \\
\end{array}$ & & & & $\begin{array}{c}\text { DESCRIPTION OF GROUP } \\
\text { SIZE }\end{array}$ & $\begin{array}{c}\text { STABILITY/ } \\
\text { AsSOCIATIONS }\end{array}$ & COMPOSITION & \\
\hline Eubalaena glacialis** & 0 & 0 & 2.57 & $\begin{array}{l}\text {-Singly, Pairs, (0) } \\
\text {-Breeding and feeding } \\
\text { grounds aggregations, (3) }\end{array}$ & $\begin{array}{l}\text {-Short (except for mother and calf) (0) } \\
\text { - Weak associations when found in } \\
\text { groups(1) }\end{array}$ & $\begin{array}{l}\text {-Pairs Mother+ Calf }(\mathbf{0}) \\
\text {-Groups segregated by sex } \\
\text { and age, Mixed }(1,2)\end{array}$ & $1,2,3$ \\
\hline Balaena mysticetus & 0 & 0 & 1 & $\begin{array}{l}\text {-Singly, Pairs, (0) } \\
\text {-Breeding and feeding } \\
\text { grounds aggregations, (3) }\end{array}$ & $\begin{array}{l}\text { Short (except for mother and calf) (0) } \\
\text { - Weak associations when found in } \\
\text { groups(1) }\end{array}$ & $\begin{array}{l}\text {-Pairs Mother+ Calf }(\mathbf{0}) \\
\text {-Groups segregated by sex } \\
\text { and age, Mixed }(1,2)\end{array}$ & $3,4,5$ \\
\hline \multicolumn{8}{|l|}{ BALAENOPTERIDAE } \\
\hline Balaenoptera borealis & 0 & 0 & 1 & $\begin{array}{l}\text {-Singly, Pairs, (0) } \\
\text {-Breeding and feeding } \\
\text { grounds aggregations, (3) }\end{array}$ & $\begin{array}{l}\text { Short (except for mother and calf) (0) } \\
\text { - Weak associations when found in groups } \\
\text { (1) }\end{array}$ & $\begin{array}{l}\text {-Pairs Mother+ Calf }(\mathbf{0}) \\
\text {-Groups segregated by sex } \\
\text { and age, Mixed }(1,2)\end{array}$ & 3,6 \\
\hline B. bonaerensis & 0 & 0 & 1 & $\begin{array}{l}\text {-Singly, Pairs, (0) } \\
\text { - Breeding and feeding } \\
\text { grounds aggregations, (3) }\end{array}$ & $\begin{array}{l}\text { Short (except for mother and calf) (0) } \\
\text { - Weak associations when found in groups } \\
\text { (1) }\end{array}$ & $\begin{array}{l}- \text { Pairs Mother+ Calf }(\mathbf{0}) \\
\text {-Groups segregated by sex } \\
\text { and age, Mixed }(1,2)\end{array}$ & 3,7 \\
\hline B. edeni/ B. brydei & 0 & 0 & 1 & $\begin{array}{l}\text {-Singly, Pairs, (0) } \\
\text {-Breeding and feeding } \\
\text { grounds aggregations, (3) }\end{array}$ & $\begin{array}{l}\text { Short (except for mother and calf) (0) } \\
\text { - Weak associations when found in groups } \\
\text { (1) }\end{array}$ & $\begin{array}{l}\text {-Pairs Mother+ Calf }(\mathbf{0}) \\
\text {-Groups segregated by sex } \\
\text { and age, Mixed }(1,2)\end{array}$ & $3,8,9$ \\
\hline B. musculus & 0 & 0 & 1 & $\begin{array}{l}\text {-Singly, Pairs, (0) } \\
\text {-Breeding and feeding } \\
\text { grounds aggregations, (3) }\end{array}$ & $\begin{array}{l}\text { Short (except for mother and calf) (0) } \\
\text { - Weak associations when found in groups } \\
\text { (1) }\end{array}$ & $\begin{array}{l}\text {-Pairs Mother+ Calf }(\mathbf{0}) \\
\text {-Groups segregated by sex } \\
\text { and age, Mixed }(1,2)\end{array}$ & 3 \\
\hline B. physalus & 0 & 0 & 1.55 & $\begin{array}{l}\text {-Singly, Pairs, (0) } \\
\text {-Small groups, (1) } \\
\text {-Breeding and feeding } \\
\text { grounds aggregations, (3) }\end{array}$ & $\begin{array}{l}\text { Short (except for mother and calf) (0) } \\
\text { - Weak associations when found in groups } \\
\text { (1) }\end{array}$ & $\begin{array}{l}\text {-Pairs Mother+ Calf }(\mathbf{0}) \\
\text {-Groups segregated by sex } \\
\text { and age, Mixed }(1,2)\end{array}$ & $3,9-13$ \\
\hline Megaptera novaeangliae & 0 & 0 & 1 & $\begin{array}{l}\text {-Singly, Pairs, (0) } \\
\text { - Breeding and feeding } \\
\text { grounds aggregations, (3) }\end{array}$ & $\begin{array}{l}\text { Short (except for mother and calf) (0) } \\
\text { - Weak associations when found in groups } \\
\text { (1) }\end{array}$ & $\begin{array}{l}\text {-Pairs Mother+ Calf }(\mathbf{0}) \\
\text {-Groups segregated by sex } \\
\text { and age, Mixed }(1,2)\end{array}$ & $3,9,14-22$ \\
\hline \multicolumn{8}{|l|}{ ESCHRICHTIDAE } \\
\hline Eschrichtius robustus & 0 & 0 & 1 & $\begin{array}{l}\text {-Singly, Pairs, (0) } \\
\text { - Breeding and feeding } \\
\text { grounds aggregations, (3) }\end{array}$ & $\begin{array}{l}\text { Short (except for mother and calf) (0) } \\
\text { - Weak associations when found in groups } \\
\text { (1) }\end{array}$ & $\begin{array}{l}- \text { Pairs Mother+ Calf }(\mathbf{0}) \\
\text {-Groups segregated by sex } \\
\text { and age, Mixed }(1,2)\end{array}$ & $3,23-24$ \\
\hline NEOBALAENIDAE & & & & & & & \\
\hline
\end{tabular}




\begin{tabular}{|c|c|c|c|c|c|c|c|}
\hline Capera marginata & 0 & 0 & 1 & $\begin{array}{l}\text {-Singly, Pairs, (0) } \\
\text { - Breeding and feeding } \\
\text { grounds aggregations, (3) }\end{array}$ & $\begin{array}{l}\text { Short (except for mother and calf) (0) } \\
\text { - Weak associations when found in groups } \\
\text { (1) }\end{array}$ & $\begin{array}{l}\text {-Pairs Mother+ Calf }(\mathbf{0}) \\
\text {-Groups segregated by sex } \\
\text { and age, Mixed }(1,2)\end{array}$ & 3 \\
\hline \multicolumn{8}{|l|}{$\begin{array}{l}\text { TOOTHED WHALES } \\
\text { (ODONTOCETI) } \\
\text { KOGIIDAE } \\
\end{array}$} \\
\hline Kogia breviceps $* *$ & $?$ & $?$ & $\sim 2$ & $\begin{array}{l}\text {-Singly, pairs (stranded } \\
\text { animals) (0) } \\
\text {-Small group (1) }\end{array}$ & $\begin{array}{l}\text { Unknown (except for the mother and calf) } \\
\text { (?) }\end{array}$ & $\begin{array}{l}- \text {-Segregated by sex and age } \\
\text { (possibly) (1) } \\
\text {-Mixed (possibly) (2) }\end{array}$ & $25-26$ \\
\hline K. simus & $?$ & $?$ & 1.87 & $\begin{array}{l}\text {-Singly (stranded animals) (0) } \\
\text {-Small groups (1) }\end{array}$ & $\begin{array}{l}\text { Unknown (except for the mother and calf) } \\
\text { (?) }\end{array}$ & $\begin{array}{l}\text {-Segregated by sex and age } \\
\text { (possibly) (1) } \\
\text {-Mixed (possibly) (2) }\end{array}$ & $25-31$ \\
\hline \multicolumn{8}{|l|}{ PHYSETERIDAE } \\
\hline Physeter macrocephalus & 1 & 3 & 22.1 & $\begin{array}{l}\text {-Solitary adult males (0) } \\
\text {-Small Female }+ \text { calves } \\
\text { (nursery groups) (1) } \\
\text {-Immature males groups (1) }\end{array}$ & $\begin{array}{l}\text {-Weak associations in immature male } \\
\text { groups (1) } \\
\text {-Long associations in Matrilineal groups (3) }\end{array}$ & $\begin{array}{l}\text {-Segregated by sex and age } \\
\text { (1) }\end{array}$ & $27,32-40$ \\
\hline \multicolumn{8}{|l|}{ ZIPHIIDAE } \\
\hline Berardius bairdii (B. anurxii) & 1 & $?$ & 7.2 & $\begin{array}{l}\text {-Small groups (male biased) } \\
\text { (1) } \\
\text {-Large Aggregations when } \\
\text { traveling (3) }\end{array}$ & $\begin{array}{l}\text { Unknown (except for the mother or father } \\
\text { and calf in this case) (?) } \\
\text { - Males possibly do parental care but it is } \\
\text { not clear what kind of associations they } \\
\text { have (?) }\end{array}$ & -Mixed (2) & $41-48$ \\
\hline Hyperoodon ampullatus & 1 & 2 & 7 & -Small groups (1) & $\begin{array}{l}\text { Fluid associations except for long-term } \\
\text { associations between males (2) }\end{array}$ & $\begin{array}{l}\text {-Segregated by sex and age } \\
\text { (1) }\end{array}$ & $49-51$ \\
\hline H. planifrons & $?$ & $?$ & 3.61 & -Small groups (1) & $\begin{array}{l}\text { Unknown (except for the mother and calf) } \\
\text { (?) }\end{array}$ & Unknown (?) & 52 \\
\hline Mesoplodon bidens & $?$ & $?$ & 3 & -Small groups (1) & $\begin{array}{l}\text { Unknown (except for the mother and calf) } \\
\text { (?) }\end{array}$ & Mixed (2) & $53-55$ \\
\hline M. densirostris & $?$ & $?$ & 3.7 & $\begin{array}{l}\text {-Singly (strandings data), } \\
\text { pairs (0) } \\
\text {-Small groups (1) } \\
\end{array}$ & $\begin{array}{l}\text { Unknown (except for the mother and calf) } \\
\text { (?) }\end{array}$ & $\begin{array}{l}\text {-Mother+calf (0) } \\
\text {-Mixed (2) }\end{array}$ & $\begin{array}{l}54-58 \\
\end{array}$ \\
\hline Ziphius cavirostris & $?$ & $?$ & 2.9 & $\begin{array}{l}\text {-Singly (strandings data), } \\
\text { pairs (0) } \\
\text {-Small groups (1) }\end{array}$ & $\begin{array}{l}\text { Unknown (except for the mother and calf) } \\
\text { (?) }\end{array}$ & $\begin{array}{l}\text {-Mother+calf (0) } \\
\text {-Mixed (2) }\end{array}$ & $27,28,56,59-62$ \\
\hline \multicolumn{8}{|l|}{ PLATANISTIDAE } \\
\hline $\begin{array}{l}\text { Platanista gangetica gangetica } \\
\text { Platanista gangetica minor }\end{array}$ & 0 & 0 & 2.45 & $\begin{array}{l}\text {-Singly, pairs (Mother+calf) } \\
\text { (most common) (0) } \\
\text {-Aggregations (3) }\end{array}$ & $\begin{array}{l}\text {-Relatively long for mother and calf }(\mathbf{0}) \\
\text {-Weak associations when found in groups } \\
\text { (1) }\end{array}$ & $\begin{array}{l}\text { Mother+calf (0) } \\
\text { Unknown (?) }\end{array}$ & $27,63-71$ \\
\hline \multicolumn{8}{|l|}{ INIIDAE } \\
\hline Inia geoffrensis & 0 & $0 / 1$ & 6.22 & $\begin{array}{l}- \text {-Singly (strandings data), } \\
\text { pairs (0) } \\
\text {-Small groups (1) } \\
\text {-Aggregations in breeding } \\
\text { and feeding grounds (3) }\end{array}$ & $\begin{array}{l}\text {-Relatively long for mother and calf }(\mathbf{0}) \\
\text {-Weak associations when found in groups } \\
\text { (1) }\end{array}$ & $\begin{array}{l}\text {-Mother+ Calf (0) } \\
\text {-Single sex (1) } \\
\text {-Mixed (2) }\end{array}$ & $27,72-78$ \\
\hline \multicolumn{8}{|l|}{ PONTOPORIDAE } \\
\hline Pontoporia blainvillei & 1 & 1 & 7.1 & -Solitary animals are rare $(0)$ & -Weak associations (described as 'fluid') & -Mixed (2) & $79-82$ \\
\hline
\end{tabular}




\begin{tabular}{|c|c|c|c|c|c|c|c|}
\hline & & & & $\begin{array}{l}\text {-Small groups (traveling, } \\
\text { feeding, socializing) (1) }\end{array}$ & (1) & & \\
\hline \multicolumn{8}{|l|}{ LIPOTIDAE } \\
\hline Lipotes vexillifer & 0 & $0 / 1$ & 3.4 & $\begin{array}{l}\text {-Singly, pairs (Mother+calf) } \\
(0) \\
\text {-Small (most common) (1) } \\
\text {-Aggregations (3) }\end{array}$ & $\begin{array}{l}\text {-Relatively long for mother and calf }(\mathbf{0}) \\
\text {-Weak associations when found in groups } \\
\text { (1) }\end{array}$ & $\begin{array}{l}\text { Mother+calf (0) } \\
\text { Unknown (?) }\end{array}$ & $27,83-86$ \\
\hline \multicolumn{8}{|l|}{ PHOCOENIDAE } \\
\hline Phocoena dioptrica & 1 & $?$ & 3 & $\begin{array}{l}\text {-Singly }(0) \\
\text {-Small groups (1) }\end{array}$ & $\begin{array}{l}\text {-Unknown (except for the mother and calf) } \\
\text { (?) }\end{array}$ & $\begin{array}{l}\text { Mother+calf (0) } \\
\text { Unknown (?) }\end{array}$ & $87-89$ \\
\hline Phocoena phocoena & 1 & 1 & 5.7 & $\begin{array}{l}- \text {-Single (0) } \\
\text {-Pairs (most common) } \\
\text {-Small groups (most } \\
\text { common) (1) } \\
\text {-Aggregations (3) } \\
\end{array}$ & $\begin{array}{l}\text {-Relatively long for mother and calf }(\mathbf{0}) \\
\text {-Weak associations when found in groups } \\
\text { (1) -described as 'fluid' }\end{array}$ & $\begin{array}{l}\text { Mother+calf (0) } \\
\text { Unknown (?) }\end{array}$ & $27,90-94$ \\
\hline P. sinus & 1 & 1 & 2 & $\begin{array}{l}\text {-Single } \\
\text {-Pairs (most common) (0) } \\
\text {-Small groups (1) }\end{array}$ & $\begin{array}{l}\text {-Relatively long for mother and calf (0) } \\
\text {-Weak associations when found in groups } \\
\text { (1) - described as 'fluid' }\end{array}$ & $\begin{array}{l}\text { Segregated by sex and age } \\
\text { (possibly) (1) }\end{array}$ & $27,95-97$ \\
\hline P. spinipinnis & 1 & $?$ & 4.5 & $\begin{array}{l}\text {-Small (most common) (1) } \\
\text {-Aggregations (3) }\end{array}$ & $\begin{array}{l}\text {-Unknown (except for the mother and calf) } \\
\text { possibly short (?) }\end{array}$ & $\begin{array}{l}\text { Mother+calf (0) } \\
\text { Unknown (?) }\end{array}$ & 27 \\
\hline Phocoenoides dalli & 1 & 1 & 7.4 & $\begin{array}{l}\text {-Single (sometimes) }(0) \\
\text {-Small groups (most } \\
\text { common) (1) } \\
\text {-Large feeding aggregations } \\
\text { (rare) (3) }\end{array}$ & $\begin{array}{l}\text {-Relatively long for mother and calf (0) } \\
\text {-Weak associations when found in groups } \\
\text { (1) -described as 'fluid' }\end{array}$ & $\begin{array}{l}\text { Segregated by sex and age } \\
\text { (possibly) (1) }\end{array}$ & $27,98-99$ \\
\hline Neophocaena phocaenoides & 1 & 1 & 3 & $\begin{array}{l}- \text {-Pairs (most common) (0) } \\
\text {-Small groups (most common } \\
\text { in Yangtzee) (1) }\end{array}$ & $\begin{array}{l}\text {-Relatively long for mother and calf (0) } \\
\text {-Weak associations when found in groups } \\
\text { (1) - described as 'undeveloped' }\end{array}$ & $\begin{array}{l}\text { Mother+calf (0) } \\
\text { Unknown (?) }\end{array}$ & $27,100-104$ \\
\hline \multicolumn{8}{|l|}{ MONODONTIDAE } \\
\hline Monodon monocerus & 1 & 3 & 3 & $\begin{array}{l}\text {-Small groups (most } \\
\text { common) (1) } \\
\text {-Large schools (2) }\end{array}$ & $\begin{array}{l}\text {-Short and fluid assocaitons (possibly in } \\
\text { large groups) (1) } \\
\text {-Matrilineal (described as possibly } \\
\text { 'matrifocal') (3) }\end{array}$ & $\begin{array}{l}\text {-Segregated by sex and age } \\
\text { (possibly) (1) } \\
\text {-Mixed (2) }\end{array}$ & $27,105-110$ \\
\hline Delphinapterus leucas & 1 & $1 / 2$ & 32.9 & $\begin{array}{l}\text {-Singly (0) } \\
\text {-Small groups (most common } \\
\text { in some areas) (1) } \\
\text {-Schools (most common in } \\
\text { some areas) (2) } \\
\text {-Large Aggregations in } \\
\text { breeding areas (3) }\end{array}$ & $\begin{array}{l}\text {-Relatively long for mother and calf ( } \mathbf{0}) \\
\text {-Weak associations when found in groups } \\
\text { (1) -described as 'fluid' }\end{array}$ & $\begin{array}{l}\text { Segregated by age and sex } \\
\text { (1) }\end{array}$ & $27,111-119$ \\
\hline \multicolumn{8}{|l|}{ DELPHINIDAE } \\
\hline Cephalorhynchus commersonii & 1 & 1 & 6.9 & -Small groups (1) & -Weak (described as ‘fission-fusion') (1) & $\begin{array}{l}\text { Mother+calf (0) } \\
\text { Unknown (?) }\end{array}$ & $27,120-121$ \\
\hline C. eutropia & 1 & 1 & 10 & -Small groups (1) & -Weak (described as ‘fission-fusion') (1) & $\begin{array}{l}\text { Mother+calf (0) } \\
\text { Unknown (?) }\end{array}$ & 120,122 \\
\hline C. hectori & 1 & 1 & 8 & -Small groups (1) & -Weak (described as 'fission-fusion') (1) & $\begin{array}{l}\text { Segregated by age and sex } \\
\text { (possibly) (1) }\end{array}$ & $123-127$ \\
\hline C. heavisidii & 1 & 1 & 3.2 & -Small groups (1) & -Weak (described as 'fission-fusion') (1) & Mother+calf $(\mathbf{0})$ & 128,120 \\
\hline
\end{tabular}




\begin{tabular}{|c|c|c|c|c|c|c|c|}
\hline & & & & & & Unknown (?) & \\
\hline Lagenorhynchus australis & $?$ & $?$ & 6.92 & $\begin{array}{l}\text {-Small groups (1) } \\
\text {-Aggregations (rare) (3) }\end{array}$ & $\begin{array}{l}\text {-Unknown (except for the mother and calf) } \\
\text { (?) } \\
\text {-Weak (described as 'fission-fusion') (1) }\end{array}$ & $\begin{array}{l}\text { Mother+calf (0) } \\
\text { Unknown (?) }\end{array}$ & $\begin{array}{l}129-131 \\
131 \mathrm{~b} \\
\end{array}$ \\
\hline L.cruciger & $?$ & $?$ & 7 & -Small groups (1) & $\begin{array}{l}\text { - Unknown (except for the mother and calf) } \\
\text { (?) }\end{array}$ & $\begin{array}{l}\text { Mother+calf (0) } \\
\text { Unknown (?) }\end{array}$ & $132-133$ \\
\hline L. obliquidens & 1 & 1 & 127.38 & $\begin{array}{l}\text {-Medium sized groups (2) } \\
\text {-Large schools (small units } \\
\text { within) }\end{array}$ & $\begin{array}{l}\text {-Weak associations (possible strong male } \\
\text { associations) }(\mathbf{1 , 2})\end{array}$ & $\begin{array}{l}\text { Segregated by age and sex } \\
\text { (1) }\end{array}$ & $27,129,134-136$ \\
\hline L. obscurus & 1 & $1 / 2$ & 86 & $\begin{array}{l}\text {-Small groups (1) } \\
\text {-Medium sized groups (most } \\
\text { common) (2) } \\
\text {-Large schools }\end{array}$ & $\begin{array}{l}\text {-Weak (1) -described as 'fission-fusion' } \\
\text {-Some long term associations (2) }\end{array}$ & $\begin{array}{l}\text { Segregated by age and sex } \\
\text { (1) }\end{array}$ & $27,137-141$ \\
\hline L. acutus & 1 & 1 & 53.2 & $\begin{array}{l}\text {-Small groups (most common } \\
\text { in some areas) (1) } \\
\text {-Medium (most common in } \\
\text { some areas) (2) } \\
\text {-Large schools }\end{array}$ & $\begin{array}{l}\text {-Unknown (except for the mother and calf) } \\
\text { possibly short (?) }\end{array}$ & $\begin{array}{l}\text { Segregated by age and sex } \\
\text { (based on strandings) (1) }\end{array}$ & $27,142-144$ \\
\hline Lissodelphins borealis & 1 & $?$ & 110.2 & $\begin{array}{l}\text {-Singly }(0) \\
\text {-Large schools (most } \\
\text { common) (2) }\end{array}$ & $\begin{array}{l}\text {-Unknown (except for the mother and calf) } \\
\text { possibly short (?) }\end{array}$ & $\begin{array}{l}\text { Mother+calf (0) } \\
\text { Unknown (?) }\end{array}$ & $27,145-147$ \\
\hline L. peronii & 1 & $?$ & 210 & $\begin{array}{l}\text {-Singly (0) } \\
\text {-Small groups (1) } \\
\text {-Large schools (most } \\
\text { common) (2) }\end{array}$ & $\begin{array}{l}\text {-Unknown (except for the mother and calf) } \\
\text { possibly short (?) }\end{array}$ & $\begin{array}{l}\text { Mother+calf (0) } \\
\text { Unknown (?) }\end{array}$ & $146,148-150$ \\
\hline Delphinus delphis & 1 & $1 / 2$ & 230.38 & $\begin{array}{l}\text {-Small subunits (within large } \\
\text { and Medium size schools) (1) } \\
\text {-Medium sized groups (most } \\
\text { common in some areas) (2) } \\
\text {-Large schools (most } \\
\text { common in some areas) } \\
\text {-Large aggregations (3) }\end{array}$ & $\begin{array}{l}\text {-Strong subunits with possible related } \\
\text { animals (3) } \\
\text {-Weak associations-described as 'highly } \\
\text { fluid fission-fusion social system' (1) }\end{array}$ & $\begin{array}{l}\text { Segregated by age and sex } \\
\text { (possibly) (1) }\end{array}$ & $28,136,151-155$ \\
\hline Delphinus capensis & 1 & $?$ & 411.69 & -Large schools (2) & $\begin{array}{l}\text {-Unknown (except for the mother and calf) } \\
\text { possibly short (?) }\end{array}$ & $\begin{array}{l}\text { Mother+calf (0) } \\
\text { Unknown (?) }\end{array}$ & 136 \\
\hline Stenella attenuata & 1 & $1 / 2$ & 360 & $\begin{array}{l}\text {-Small groups (1) } \\
\text {-Medium sized groups (2) } \\
\text {-Large schools }\end{array}$ & $\begin{array}{l}\text {-Strong associations (possibly within male } \\
\text { groups) (2) } \\
\text {-Weak associations (described as 'fluid') } \\
\text { (1) }\end{array}$ & $\begin{array}{l}\text { Segregated by age and sex } \\
\text { (1) }\end{array}$ & $27-28,156-162$ \\
\hline S. clymene & 1 & $?$ & 97.4 & $\begin{array}{l}\text {-Small groups (1) } \\
\text {-Medium sized groups (2) } \\
\text {-Large schools }\end{array}$ & $\begin{array}{l}\text {-Unknown (except for the mother and calf) } \\
\text { possibly short (?) }\end{array}$ & $\begin{array}{l}\text { Segregated by age and sex } \\
\text { (based on strandings) (1) }\end{array}$ & $\begin{array}{l}27-28,136,163- \\
167\end{array}$ \\
\hline S. frontalis & 1 & $1 / 2$ & 10 & -Small groups (1) & $\begin{array}{l}\text {-Strong associations (within male groups) } \\
\text { (2) } \\
\text {-Weak associations (described as 'fluid') } \\
\text { (1) }\end{array}$ & $\begin{array}{l}\text { Segregated by age and sex } \\
\text { (1) }\end{array}$ & $27,168-173$ \\
\hline S. coeruleoalba & 1 & $1 / 2$ & 302 & $\begin{array}{l}- \text {-Small (most common in } \\
\text { some areas) (1) } \\
\text {-Medium (most common in } \\
\text { some areas) (2) } \\
\text {-Large schools (most }\end{array}$ & $\begin{array}{l}\text {-Strong associations (possibly within male } \\
\text { groups) (2) } \\
\text {-Weak associations (described as 'fluid') } \\
\text { (1) }\end{array}$ & $\begin{array}{l}\text {-Segregated by age and sex } \\
\text { (1) } \\
\text {-Mixed (2) }\end{array}$ & $\begin{array}{l}27-28,136,174- \\
175\end{array}$ \\
\hline
\end{tabular}




\begin{tabular}{|c|c|c|c|c|c|c|c|}
\hline & & & & common in some areas) & & & \\
\hline S. longirostris & 1 & $1 / 2$ & 147.74 & -Large schools (2) & $\begin{array}{l}\text {-Weak associations (described as 'fluid') } \\
\text { (1) } \\
\text {-Strong (described as 'strongly bonded') for } \\
\text { other group members not necessarily just } \\
\text { males) (2) }\end{array}$ & $\begin{array}{l}\text {-Segregation by sex and age } \\
\text { (1) }\end{array}$ & $\begin{array}{l}27-28,136,177, \\
179,180,178,176, \\
181,\end{array}$ \\
\hline Tursiops truncatus & 1 & $1 / 2$ & $92-2$ & $\begin{array}{l}\text {-Small groups (most common } \\
\text { in some areas) (1) } \\
\text {-Medium sized groups (most } \\
\text { common in some areas) (2) } \\
\text {-Large schools (most } \\
\text { common in offshore ecotype) }\end{array}$ & $\begin{array}{l}\text {-Strong (males coalitions and alliances) (2) } \\
\text {-Weak (described as 'fission-fusion') (1) }\end{array}$ & $\begin{array}{l}\text {-Segregation by sex and age } \\
\text { (1) }\end{array}$ & $\begin{array}{l}27-28,136,182- \\
199\end{array}$ \\
\hline Lagenodelphis hosei & 1 & 2 & 440.05 & -Large schools (2) & $\begin{array}{l}\text {-Strong (described as more strong than } \\
\text { other social dolphins like Stenella) (2) }\end{array}$ & $\begin{array}{l}\text {-Mixed (based on } \\
\text { strandings) (2) }\end{array}$ & $27,136,200-204$ \\
\hline Sousa chinensis & 1 & $1 / 2$ & 14.9 & $\begin{array}{l}\text {-Solitary large adults }(0) \\
\text {-Small groups (most } \\
\text { common) (1) } \\
\text {-Medium sized groups (rare) }\end{array}$ & $\begin{array}{l}\text {-Weak (described as 'fluid with short- } \\
\text { lasting associations') (1) } \\
\text {-Strong mother +calf (0) } \\
\text {-Strong (affiliations in stable groups from } \\
\text { Mozambique) (2) }\end{array}$ & $\begin{array}{l}\text {-Segregation by sex and age } \\
\text { (1) } \\
\text {-Mixed (all age classes) (2) }\end{array}$ & $27,205-210$ \\
\hline $\begin{array}{l}\text { Sotalia fluviatilis (riverine) } \\
\text { Sotalia guianensis (marine) }\end{array}$ & 1 & 2 & 13 & $\begin{array}{l}\text {-Singly (both species) (0) } \\
\text {-Small (riverine) (most } \\
\text { common) (1) } \\
\text {-Medium (marine) (2) } \\
\text {-Large feeding aggregations } \\
\text { (marine) (3) }\end{array}$ & $\begin{array}{l}\text {-Weak associations (1) } \\
\text {-'Family' (2adults+calf) described in the } \\
\text { marine species (2) }\end{array}$ & -Mixed (2) & $211-218$ \\
\hline Steno bredanensis & 1 & ? & 40 & $\begin{array}{l}\text {-Small (most common in } \\
\text { some areas) (1) } \\
\text {-Medium (most common in } \\
\text { some areas) (2) } \\
\text {-Large aggregations (3) }\end{array}$ & $\begin{array}{l}\text { Unknown (except for the mother and calf) } \\
\text { (?) }\end{array}$ & $\begin{array}{l}\text {-Mixed (based on strandings } \\
\text { but largely unknown) (2) }\end{array}$ & $\begin{array}{l}27-28,136,219- \\
223\end{array}$ \\
\hline Feresa attenuata & 1 & $?$ & 30.12 & $\begin{array}{l}\text {-Small (more common in } \\
\text { some areas) (1) } \\
\text {-Medium sized groups (more } \\
\text { common in some areas) (2) } \\
\text {-Large schools (rare) } \\
\end{array}$ & $\begin{array}{l}\text {-Strong (possibly similar to other } \\
\text { globicephaliinids were individuals are } \\
\text { related) (3) }\end{array}$ & $\begin{array}{l}\text {-Mixed (based on strandings } \\
\text { but largely unknown) (2) }\end{array}$ & $27,136,224-227$ \\
\hline Globicephala macrorhynchus & 1 & 3 & 41.1 & $\begin{array}{l}\text {-Small groups (1) } \\
\text {-Medium sized groups (2) }\end{array}$ & $\begin{array}{l}\text {-Matrilineal (natal philopatry, males live } \\
\text { the group) (3) }\end{array}$ & -Mixed (2) & $\begin{array}{l}27-28,45,136, \\
228-234\end{array}$ \\
\hline G. melas & 1 & 3 & 84.5 & $\begin{array}{l}\text {-Medium sized groups (2) } \\
\text {-Large schools }\end{array}$ & $\begin{array}{l}\text {-Matrilineal (natal philopatry, males live } \\
\text { the group) (3) } \\
\text {-Weak (some evidence of short term } \\
\text { associations) (1) }\end{array}$ & -Mixed (2) & $\begin{array}{l}27,228-229,231- \\
232,234,235-238\end{array}$ \\
\hline Grampus griseus & 1 & $1 / 2$ & 63 & $\begin{array}{l}\text {-Small (more common in } \\
\text { some areas) (1) } \\
\text {-Medium (more common in } \\
\text { some areas)(2) } \\
\text {-Large schools (rare) }\end{array}$ & $\begin{array}{l}\text {-Possibly strong, calves tend to stay longer } \\
\text { than non-globicephaliinids dolphins. (2) } \\
\text {-Natal philopatry, males move between } \\
\text { groups) (3) }\end{array}$ & $\begin{array}{l}\text {-Mixed (2) } \\
\text {-Maybe some segregation by } \\
\text { age (1) }\end{array}$ & $\begin{array}{l}27-28,136,234, \\
239-243\end{array}$ \\
\hline Peponocephala electra & 1 & 3 & 257.7 & $\begin{array}{l}\text {-Large schools (more } \\
\text { common) (2) }\end{array}$ & $\begin{array}{l}\text {-Strong (described as 'strong social bonds') } \\
\text { (2) }\end{array}$ & $\begin{array}{l}\text { Mother+calf (0) } \\
\text { Unknown (?) }\end{array}$ & $\begin{array}{l}27,136,234,244- \\
246\end{array}$ \\
\hline
\end{tabular}




\begin{tabular}{|l|l|l|l|l|l|l|}
\hline Pseudorca crassidens & 1 & 3 & 36.16 & $\begin{array}{l}\text {-Small groups (1) } \\
\text {-Medium (more common in } \\
\text { some areas) (2) } \\
\text {-Large schools }\end{array}$ & $\begin{array}{l}\text {-Strong (described as 'strong affiliative } \\
\text { behavior' in stranded animals) (2) }\end{array}$ & $\begin{array}{l}\text {-Mixed (based on } \\
\text { strandings) (2) }\end{array}$ \\
\hline $\begin{array}{l}\text { Orcaella brevirostris (riverine) } \\
\text { Orcaella heinsohni (marine) }\end{array}$ & 1 & 0 & 3.5 & $\begin{array}{l}\text {-Small (most common) (1) } \\
\text {-Medium sized groups (rare) } \\
\text { (2) }\end{array}$ & $\begin{array}{l}\text {-Weak (described as 'frequency mixing' (1) } \\
\text { 247-250 }\end{array}$ & $\begin{array}{l}\text { Mother+calf (0) } \\
\text { Unknown (?) }\end{array}$ \\
\hline Orcinus orca & 1 & 3 & 12 & $\begin{array}{l}\text {-Single (mainly males are } \\
\text { infrequent) (0) } \\
\text {-Small to Medium ('fish } \\
\text { eating') (1) } \\
\text {-Small ('mammal eating') (1) } \\
\text {-Large aggregations (3) }\end{array}$ & $\begin{array}{l}\text {-Matrilineal with natal philopatry in fish } \\
\text { eating orcas (3) } \\
\text {-Two generation matrilineal in mammal } \\
\text { eating orcas (3) }\end{array}$ & $\begin{array}{l}\text {-Mixed (2) } \\
\text { (2) }\end{array}$ \\
\hline
\end{tabular}

**Species which part of information comes from the sister species 


\section{APPENDIX 6}

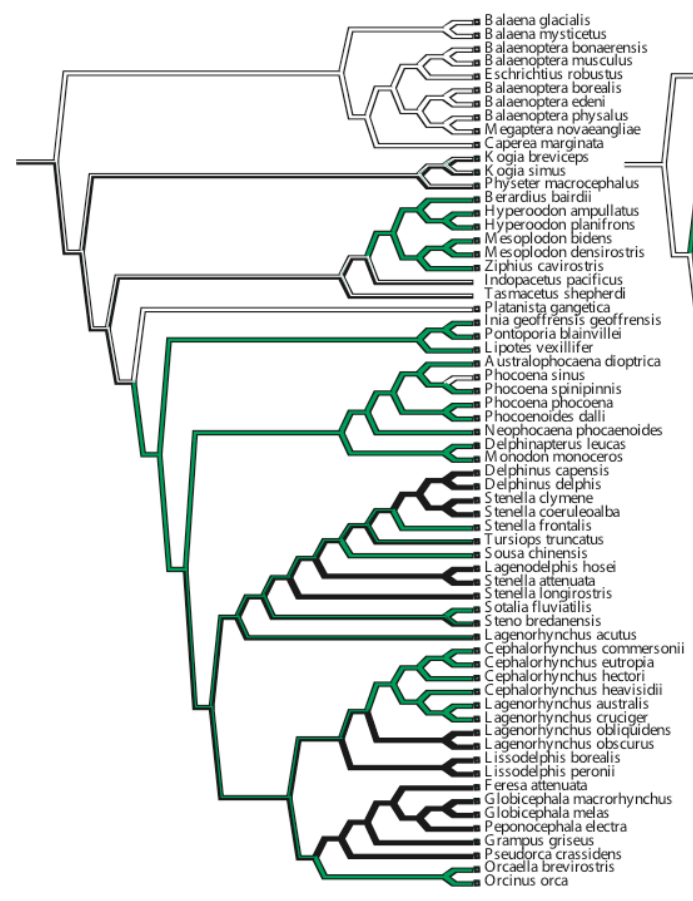

$\square$ solitary or pairs

small groups
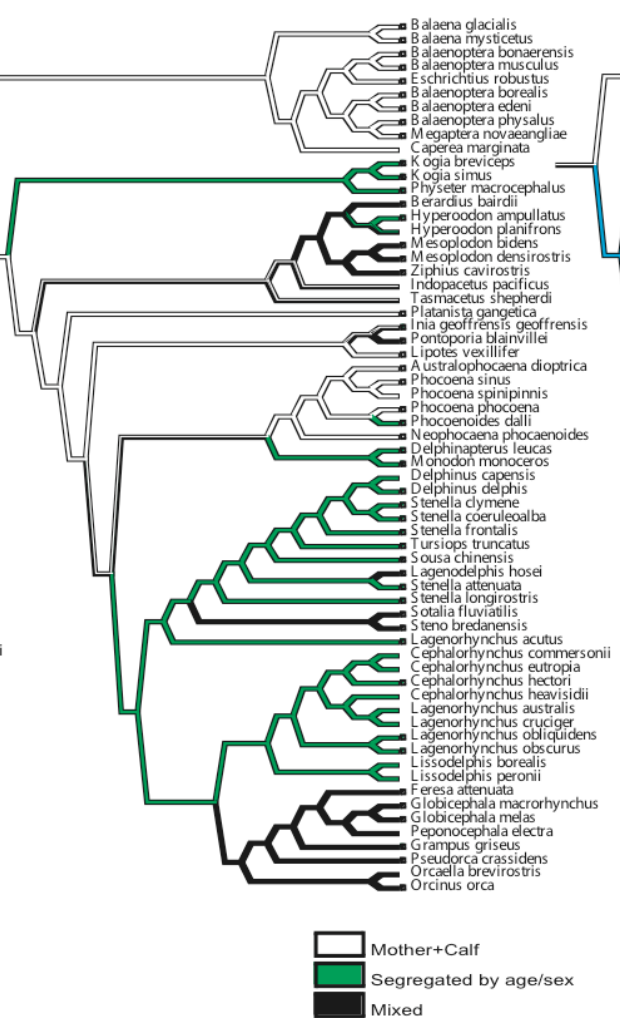

b. Group Composition
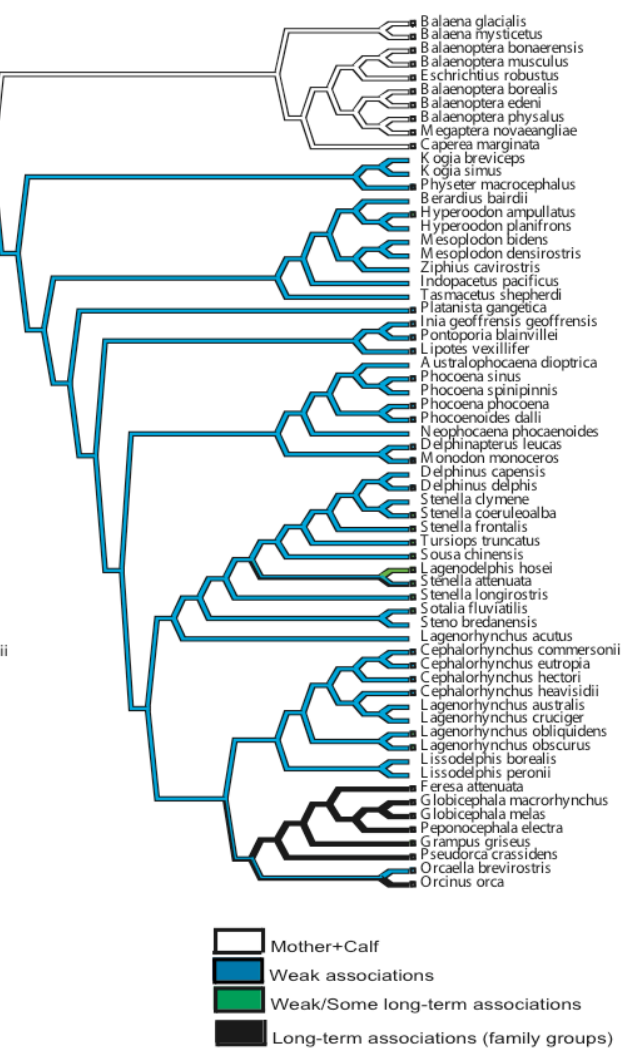

c. Group Stability/Associations

Optimization of components of sociality. This figure shows social components optimization ( $\mathrm{a}=$ group size, $\mathrm{b}=$ group composition, $\mathrm{c}=$ group stability/association patterns) on the preferred phylogeny. Note that this optimization contains polymorphic species and thus family based group like Physeter and Monodon and species with long-term associations between non-related group members are all optimized using the lowest state of sociality. 
APPENDIX 7. Cetacean tonal sound acoustic parameters. This table reviews published data on cetacean tonal sound acoustic parameters. Numbers in bold correspond to the preferred value used in the optimizations (see Methods).

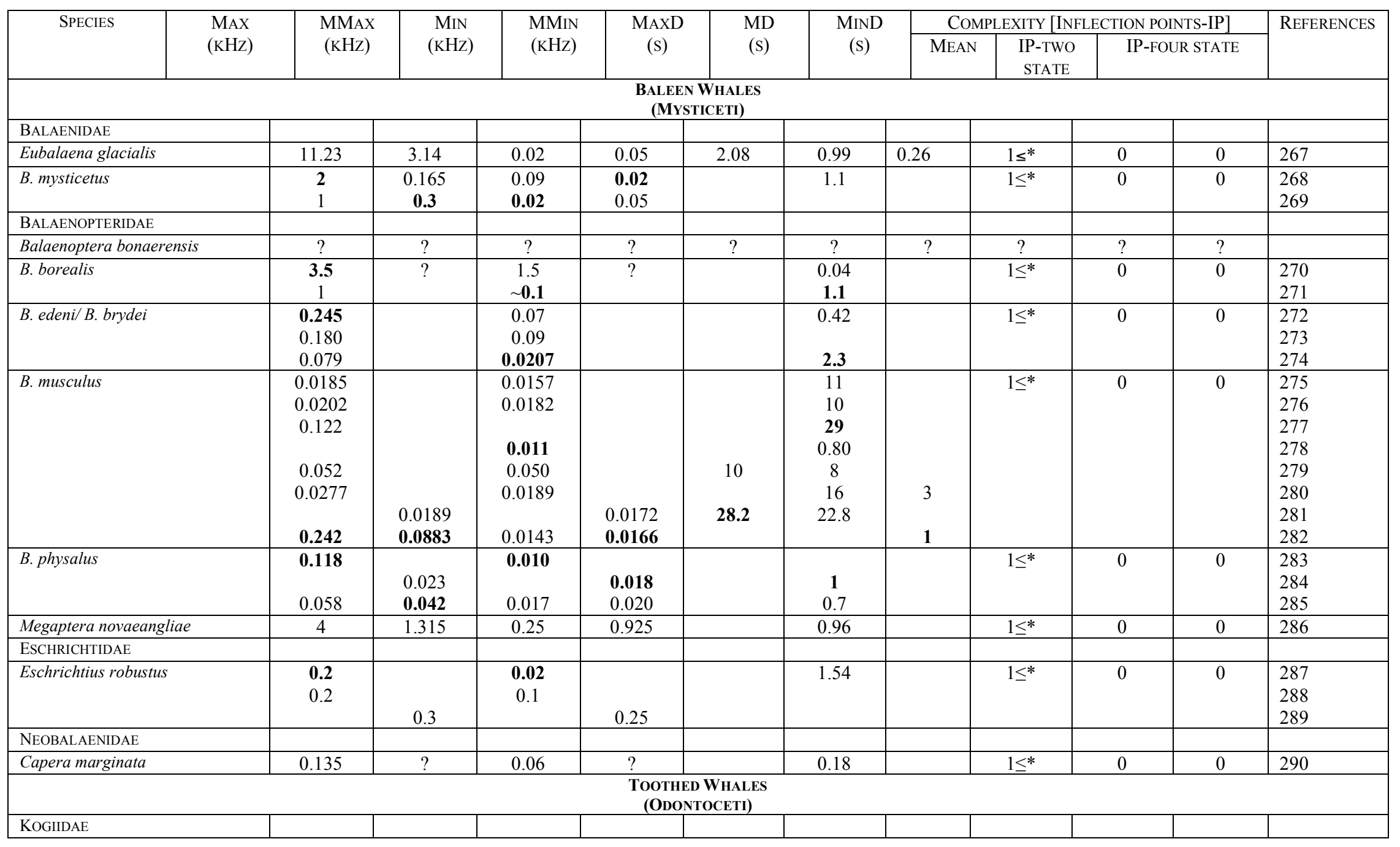




\begin{tabular}{|c|c|c|c|c|c|c|c|c|c|c|c|}
\hline Kogia breviceps & $?$ & $?$ & $?$ & $?$ & $?$ & $?$ & $?$ & $?$ & $?$ & $?$ & \\
\hline K. simus & $?$ & $?$ & $?$ & $?$ & $?$ & $?$ & $?$ & $?$ & $?$ & $?$ & \\
\hline \multicolumn{12}{|l|}{ PHYSETERIDAE } \\
\hline Physeter macrocephalus & $\mathrm{n} / \mathrm{a}$ & $\mathrm{n} / \mathrm{a}$ & $\mathrm{n} / \mathrm{a}$ & $\mathrm{n} / \mathrm{a}$ & $\mathrm{n} / \mathrm{a}$ & $\mathrm{n} / \mathrm{a}$ & $\mathrm{n} / \mathrm{a}$ & $\mathrm{n} / \mathrm{a}$ & $\mathrm{n} / \mathrm{a}$ & $\mathrm{n} / \mathrm{a}$ & \\
\hline \multicolumn{12}{|l|}{ ZIPHIIDAE } \\
\hline Berardius bairdii (B. anurxii) & 8.0 & $?$ & 4 & $?$ & $\sim 3.5$ & & $\sim 2$ & $\sim 3$ & 1 & 2 & 46 \\
\hline Hyperoodon ampullatus & $\mathrm{n} / \mathrm{a}$ & $\mathrm{n} / \mathrm{a}$ & $\mathrm{n} / \mathrm{a}$ & $\mathrm{n} / \mathrm{a}$ & $\mathrm{n} / \mathrm{a}$ & $\mathrm{n} / \mathrm{a}$ & $\mathrm{n} / \mathrm{a}$ & $\mathrm{n} / \mathrm{a}$ & $\mathrm{n} / \mathrm{a}$ & $\mathrm{n} / \mathrm{a}$ & \\
\hline H. planifrons & $?$ & $?$ & $?$ & $?$ & $?$ & $?$ & $?$ & $?$ & $?$ & $?$ & \\
\hline Mesoplodon bidens & $?$ & $?$ & $?$ & $?$ & $?$ & $?$ & $?$ & $?$ & $?$ & $?$ & \\
\hline M. densirostris & $?$ & $?$ & $?$ & $?$ & $?$ & $?$ & $?$ & $?$ & $?$ & $?$ & \\
\hline Ziphius cavirostris & $?$ & $?$ & $?$ & $?$ & $?$ & $?$ & $?$ & $?$ & $?$ & $?$ & \\
\hline \multicolumn{12}{|l|}{ PLATANISTIDAE } \\
\hline Platanista gangetica & $?$ & $?$ & $?$ & $?$ & $?$ & $?$ & $?$ & $?$ & $?$ & $?$ & \\
\hline \multicolumn{12}{|l|}{ INIIDAE } \\
\hline Inia geoffrensis & $\begin{array}{c}\mathbf{4 8 . 1 0} \\
5.16 \\
13\end{array}$ & $\begin{array}{c}\mathbf{2 4 . 7 1} \\
2.97\end{array}$ & $\begin{array}{c}5.03 \\
0.22 \\
3\end{array}$ & $\begin{array}{c}15.06 \\
\mathbf{2 . 5 4}\end{array}$ & $\begin{array}{c}0.080 \\
\mathbf{4 . 4 2}\end{array}$ & $\begin{array}{c}0.009 \\
\mathbf{1 . 1 4}\end{array}$ & $\begin{array}{c}\mathbf{0 . 0 0 2} \\
0.16\end{array}$ & 1.05 & 1 & 1 & $\begin{array}{l}291 \\
292-293 \\
294\end{array}$ \\
\hline \multicolumn{12}{|l|}{ PONTOPORIDAE } \\
\hline Pontoporia blainvillei & $?$ & $?$ & $?$ & $?$ & $?$ & $?$ & $?$ & $?$ & & & \\
\hline \multicolumn{12}{|l|}{ LIPOTIDAE } \\
\hline Lipotes vexillifer & $\begin{array}{l}4.5 \\
4.6\end{array}$ & $\begin{array}{c}5.84 \\
6.1\end{array}$ & $\begin{array}{l}3.8 \\
3.8\end{array}$ & $\begin{array}{l}5.0 \\
4.9\end{array}$ & $\begin{array}{l}\mathbf{1 . 8} \\
1.6\end{array}$ & $\begin{array}{l}1 \\
1\end{array}$ & $\begin{array}{l}\mathbf{0 . 4} \\
0.5\end{array}$ & 0.72 & 1 & 0 & $\begin{array}{l}295 \\
296\end{array}$ \\
\hline \multicolumn{12}{|l|}{ PHOCOENIDAE } \\
\hline Phocoena dioptrica & $\mathrm{n} / \mathrm{a}$ & $\mathrm{n} / \mathrm{a}$ & $\mathrm{n} / \mathrm{a}$ & $\mathrm{n} / \mathrm{a}$ & $\mathrm{n} / \mathrm{a}$ & $\mathrm{n} / \mathrm{a}$ & $\mathrm{n} / \mathrm{a}$ & $\mathrm{n} / \mathrm{a}$ & $\mathrm{n} / \mathrm{a}$ & $\mathrm{n} / \mathrm{a}$ & \\
\hline Phocoena phocoena & $\mathrm{n} / \mathrm{a}$ & $\mathrm{n} / \mathrm{a}$ & $\mathrm{n} / \mathrm{a}$ & $\mathrm{n} / \mathrm{a}$ & $\mathrm{n} / \mathrm{a}$ & $\mathrm{n} / \mathrm{a}$ & $\mathrm{n} / \mathrm{a}$ & $\mathrm{n} / \mathrm{a}$ & $\mathrm{n} / \mathrm{a}$ & $\mathrm{n} / \mathrm{a}$ & \\
\hline P. sinus & $\mathrm{n} / \mathrm{a}$ & $\mathrm{n} / \mathrm{a}$ & $\mathrm{n} / \mathrm{a}$ & $\mathrm{n} / \mathrm{a}$ & $\mathrm{n} / \mathrm{a}$ & $\mathrm{n} / \mathrm{a}$ & $\mathrm{n} / \mathrm{a}$ & $\mathrm{n} / \mathrm{a}$ & $\mathrm{n} / \mathrm{a}$ & $\mathrm{n} / \mathrm{a}$ & \\
\hline P. spinipinnis & $\mathrm{n} / \mathrm{a}$ & $\mathrm{n} / \mathrm{a}$ & $\mathrm{n} / \mathrm{a}$ & $\mathrm{n} / \mathrm{a}$ & $\mathrm{n} / \mathrm{a}$ & $\mathrm{n} / \mathrm{a}$ & $\mathrm{n} / \mathrm{a}$ & $\mathrm{n} / \mathrm{a}$ & $\mathrm{n} / \mathrm{a}$ & $\mathrm{n} / \mathrm{a}$ & \\
\hline Phocoenoides dalli & $\mathrm{n} / \mathrm{a}$ & $\mathrm{n} / \mathrm{a}$ & $\mathrm{n} / \mathrm{a}$ & $\mathrm{n} / \mathrm{a}$ & $\mathrm{n} / \mathrm{a}$ & $\mathrm{n} / \mathrm{a}$ & $\mathrm{n} / \mathrm{a}$ & $\mathrm{n} / \mathrm{a}$ & $\mathrm{n} / \mathrm{a}$ & $\mathrm{n} / \mathrm{a}$ & \\
\hline Neophocaena phocaenoides & $\mathrm{n} / \mathrm{a}$ & $\mathrm{n} / \mathrm{a}$ & $\mathrm{n} / \mathrm{a}$ & $\mathrm{n} / \mathrm{a}$ & $\mathrm{n} / \mathrm{a}$ & $\mathrm{n} / \mathrm{a}$ & $\mathrm{n} / \mathrm{a}$ & $\mathrm{n} / \mathrm{a}$ & $\mathrm{n} / \mathrm{a}$ & $\mathrm{n} / \mathrm{a}$ & \\
\hline \multicolumn{12}{|l|}{ MONODONTIDAE } \\
\hline Monodon monocerus & $\begin{array}{c}\mathbf{1 8} \\
10 \\
8.84\end{array}$ & 7.18 & $\begin{array}{c}\mathbf{0 . 3} \\
5 \\
0.360\end{array}$ & 0.718 & $\begin{array}{c}\mathbf{6} \\
0.85 \\
1.26\end{array}$ & 1.19 & $\begin{array}{c}\mathbf{0 . 0 5} \\
0.1 \\
0.68\end{array}$ & $\begin{array}{l}1 \leq * \\
1 \leq * \\
1 \leq *\end{array}$ & 0 & $?$ & $\begin{array}{l}297 \\
298 \\
299\end{array}$ \\
\hline Delphinapterus leucas & $\begin{array}{c}\mathbf{1 9 . 6} \\
15.8\end{array}$ & $\begin{array}{c}6.8 \\
4.33 \\
\mathbf{1 1 . 6 5} \\
\end{array}$ & $\begin{array}{l}\mathbf{0 . 2} \\
0.4\end{array}$ & $\begin{array}{c}3.8 \\
3.38 \\
1.99 \\
\end{array}$ & $\begin{array}{c}3.92 \\
3.2\end{array}$ & $\begin{array}{l}1.06 \\
0.75 \\
\mathbf{1 . 1 2} \\
\end{array}$ & $\begin{array}{l}\mathbf{0 . 0 1} \\
0.05\end{array}$ & $\begin{array}{l}1>^{*} \\
1>* \\
13.5 \\
\end{array}$ & 1 & 3 & $\begin{array}{l}300 \\
301 \\
302 \\
\end{array}$ \\
\hline \multicolumn{12}{|l|}{ DELPHINIDAE } \\
\hline Cephalorhynchus commersonii & $\mathrm{n} / \mathrm{a}$ & $\mathrm{n} / \mathrm{a}$ & $\mathrm{n} / \mathrm{a}$ & $\mathrm{n} / \mathrm{a}$ & $\mathrm{n} / \mathrm{a}$ & $\mathrm{n} / \mathrm{a}$ & $\mathrm{n} / \mathrm{a}$ & $\mathrm{n} / \mathrm{a}$ & $\mathrm{n} / \mathrm{a}$ & $\mathrm{n} / \mathrm{a}$ & \\
\hline
\end{tabular}




\begin{tabular}{|c|c|c|c|c|c|c|c|c|c|c|c|}
\hline C. eutropia & $\mathrm{n} / \mathrm{a}$ & $\mathrm{n} / \mathrm{a}$ & $\mathrm{n} / \mathrm{a}$ & $\mathrm{n} / \mathrm{a}$ & $\mathrm{n} / \mathrm{a}$ & $\mathrm{n} / \mathrm{a}$ & $\mathrm{n} / \mathrm{a}$ & $\mathrm{n} / \mathrm{a}$ & $\mathrm{n} / \mathrm{a}$ & $\mathrm{n} / \mathrm{a}$ & \\
\hline C. hectori & $\mathrm{n} / \mathrm{a}$ & $\mathrm{n} / \mathrm{a}$ & $\mathrm{n} / \mathrm{a}$ & $\mathrm{n} / \mathrm{a}$ & $\mathrm{n} / \mathrm{a}$ & $\mathrm{n} / \mathrm{a}$ & $\mathrm{n} / \mathrm{a}$ & $\mathrm{n} / \mathrm{a}$ & $\mathrm{n} / \mathrm{a}$ & $\mathrm{n} / \mathrm{a}$ & \\
\hline C. heavisidii & $\mathrm{n} / \mathrm{a}$ & $\mathrm{n} / \mathrm{a}$ & $\mathrm{n} / \mathrm{a}$ & $\mathrm{n} / \mathrm{a}$ & $\mathrm{n} / \mathrm{a}$ & $\mathrm{n} / \mathrm{a}$ & $\mathrm{n} / \mathrm{a}$ & $\mathrm{n} / \mathrm{a}$ & $\mathrm{n} / \mathrm{a}$ & $\mathrm{n} / \mathrm{a}$ & \\
\hline Lagenorhynchus australis & $\mathrm{n} / \mathrm{a}$ & $\mathrm{n} / \mathrm{a}$ & $\mathrm{n} / \mathrm{a}$ & $\mathrm{n} / \mathrm{a}$ & $\mathrm{n} / \mathrm{a}$ & $\mathrm{n} / \mathrm{a}$ & $\mathrm{n} / \mathrm{a}$ & $\mathrm{n} / \mathrm{a}$ & $\mathrm{n} / \mathrm{a}$ & $\mathrm{n} / \mathrm{a}$ & \\
\hline L.cruciger & $?$ & $?$ & $?$ & $?$ & $?$ & $?$ & $?$ & $?$ & $?$ & $?$ & \\
\hline L. obliquidens & $\sim 13$ & $\sim 10$ & $\sim 1$ & $?$ & 0.2 & 0.7 & 1.2 & $\sim>1$ & 1 & 0 & 303 \\
\hline L. obscurus & 27.3 & $\begin{array}{l}\mathbf{1 6 . 4 9} \\
13.22\end{array}$ & 1.04 & $\begin{array}{l}8.11 \\
8.15\end{array}$ & $\begin{array}{l}\mathbf{3 . 1 4} \\
1.04\end{array}$ & $\begin{array}{l}\mathbf{1 . 0 3} \\
0.535\end{array}$ & $\begin{array}{c}0.18 \\
\mathbf{0 . 0 1 4}\end{array}$ & 1.97 & 1 & 1 & $\begin{array}{l}292 \\
304 \\
\end{array}$ \\
\hline L. acutus & $?$ & 12.14 & $?$ & 8.21 & & 0.5 & & 0.92 & 1 & 0 & 305 \\
\hline Lissodelphins borealis & $?$ & $?$ & $?$ & $?$ & $?$ & $?$ & $?$ & $?$ & $?$ & $?$ & \\
\hline L. peronii & $?$ & $?$ & $?$ & $?$ & $?$ & $?$ & $?$ & $?$ & $?$ & $?$ & \\
\hline Delphinus delphis & 19.8 & $\begin{array}{c}11.65 \\
\mathbf{1 3 . 6}\end{array}$ & 4.8 & $\begin{array}{c}6.42 \\
7.4\end{array}$ & & 0.8 & & $\begin{array}{l}1>^{*} \\
1.2\end{array}$ & 1 & 1 & $\begin{array}{l}306 \\
307\end{array}$ \\
\hline Delphinus capensis & $?$ & 15.5 & $?$ & 7.7 & & 0.70 & & 1.3 & 1 & 1 & 307 \\
\hline Stenella attenuata & 21.4 & $\begin{array}{c}15.72 \\
\mathbf{1 8 . 7}\end{array}$ & 3.13 & $\begin{array}{c}8.73 \\
8.2\end{array}$ & 1.95 & $\begin{array}{l}0.53 \\
0.9\end{array}$ & 0.09 & $\begin{array}{c}0.70 \\
1.9\end{array}$ & 1 & 1 & $\begin{array}{l}292 \\
307\end{array}$ \\
\hline S. clymene & $?$ & \begin{tabular}{|c|}
$\mathbf{1 9 . 2}$ \\
13.62 \\
\end{tabular} & $?$ & $\begin{array}{l}\mathbf{6 . 3 3} \\
9.25 \\
\end{array}$ & & $\begin{array}{l}\mathbf{0 . 6 1} \\
0.41 \\
\end{array}$ & & $1>^{*}$ & 1 & $?$ & $\begin{array}{l}308 \\
309 \\
\end{array}$ \\
\hline S. frontalis & 19.8 & 16.04 & 5 & 7.91 & 2.07 & 0.82 & 0.08 & 3.43 & 1 & 3 & 292 \\
\hline S. coeruleoalba & 22.99 & $\begin{array}{c}11.53 \\
\mathbf{1 4 . 8}\end{array}$ & 1.1 & $\begin{array}{c}6.84 \\
8.1\end{array}$ & & $\begin{array}{l}0.54 \\
\mathbf{0 . 8}\end{array}$ & & $\begin{array}{l}1.3 \\
1.9\end{array}$ & 1 & 1 & $\begin{array}{l}310 \\
307\end{array}$ \\
\hline S. longirostris & $\begin{array}{c}22.5 \\
\\
24 \\
\mathbf{2 5 . 2 5} \\
23.04\end{array}$ & $\begin{array}{c}15.2 \\
13.7 \\
16.5 \\
\mathbf{1 7 . 5 6} \\
16.8 \\
14.32\end{array}$ & 3.91 & $\begin{array}{c}9.03 \\
9.1 \\
9.99 \\
9.66 \\
10.19 \\
\mathbf{8 . 7 6} \\
\end{array}$ & $\begin{array}{l}3.35 \\
\mathbf{4 . 4 9} \\
1.87 \\
\end{array}$ & $\begin{array}{l}\mathbf{0 . 7 5} \\
0.6 \\
0.72 \\
0.49 \\
0.61 \\
0.43\end{array}$ & $\begin{array}{c}0.10 \\
0.016 \\
0.013 \\
\mathbf{0 . 0 4 0}\end{array}$ & $\begin{array}{c}1.07 \\
\mathbf{1 . 9}\end{array}$ & 1 & 1 & $\begin{array}{l}292 \\
307 \\
311 \\
312 \\
313 \\
305 \\
\end{array}$ \\
\hline Tursiops truncatus & $\begin{array}{c}\mathbf{4 1} \\
21.6\end{array}$ & $\begin{array}{c}\mathbf{1 7 . 2} \\
11.35 \\
11.95 \\
16.24 \\
\end{array}$ & $\begin{array}{l}1.86 \\
0.94\end{array}$ & $\begin{array}{c}7.4 \\
\mathbf{5 . 4 6} \\
7.33 \\
\end{array}$ & $\begin{array}{l}3.20 \\
3.20\end{array}$ & $\begin{array}{l}0.86 \\
\mathbf{1 . 4} \\
0.70 \\
0.75 \\
1.3 \\
\end{array}$ & $\begin{array}{l}0.05 \\
0.05\end{array}$ & $\begin{array}{c}3.7 \\
1.86 \\
2.14 \\
2.86 \\
\end{array}$ & 1 & 3 & $\begin{array}{l}314 \\
307 \\
292 \\
315 \\
305 \\
\end{array}$ \\
\hline Lagenodelphis hosei & $\begin{array}{l}24.0 \\
18.3 \\
13.4\end{array}$ & $\begin{array}{l}\mathbf{1 6 . 9} \\
14.9\end{array}$ & $\begin{array}{l}4.3 \\
6.6\end{array}$ & $\begin{array}{c}9.36 \\
11 \\
\mathbf{7 . 6 4}\end{array}$ & 0.5 & $\begin{array}{l}\mathbf{0 . 7 7} \\
0.46\end{array}$ & 0.4 & 0.80 & 1 & 0 & $\begin{array}{l}316 \\
317 \\
318 \\
\end{array}$ \\
\hline Sousa chinensis & $\begin{array}{l}\mathbf{2 2} \\
20\end{array}$ & 16.3 & $\begin{array}{c}\mathbf{0 . 9} \\
3 \\
1.2\end{array}$ & 4.5 & 1.3 & $\begin{array}{l}\mathbf{1 . 1} \\
0.2 \\
0.13\end{array}$ & 0.01 & $1>*$ & 1 & $?$ & $\begin{array}{l}319 \\
320 \\
321\end{array}$ \\
\hline Sotalia fluviatilis** & 23.9 & $\begin{array}{l}19.95 \\
15.41\end{array}$ & $\begin{array}{l}1.34 \\
3.65\end{array}$ & $\begin{array}{l}7.21 \\
10.2\end{array}$ & $\begin{array}{r}\mathbf{1 . 0 6 4} \\
1.04 \\
\end{array}$ & $\begin{array}{l}0.381 \\
0.41\end{array}$ & $\begin{array}{c}0.038 \\
0.06\end{array}$ & $\begin{array}{l}0.77 \\
\mathbf{1 . 3 8}\end{array}$ & 1 & 1 & $\begin{array}{l}322 \\
292,293\end{array}$ \\
\hline
\end{tabular}




\begin{tabular}{|c|c|c|c|c|c|c|c|c|c|c|c|}
\hline & $\begin{array}{c}\mathbf{3 8 . 2 5} \\
18 \\
17.49\end{array}$ & $\begin{array}{c}\mathbf{2 1 . 3 2} \\
13 \\
13.312 \\
15.65\end{array}$ & $\begin{array}{c}2.714 \\
\mathbf{0 . 5} \\
1.031\end{array}$ & $\begin{array}{c}13.14 \\
7.6 \\
10.521 \\
9.18\end{array}$ & $\begin{array}{c}0.852 \\
2.2\end{array}$ & $\begin{array}{l}0.103 \\
\mathbf{0 . 7 9} \\
0.63\end{array}$ & $\begin{array}{c}0.01 \\
\mathbf{0 . 0 0 9}\end{array}$ & $\begin{array}{l}0.7 \\
1.3\end{array}$ & & & $\begin{array}{l}\text { Authors } \\
\text { unpublished } \\
\text { data } \\
323 \\
324 \\
325\end{array}$ \\
\hline Steno bredanensis & 7.0 & 9.1 & 4 & 6.03 & & 0.6 & & 1.3 & 1 & 1 & $\begin{array}{l}326 \\
307 \\
\end{array}$ \\
\hline Feresa attenuata & $?$ & $?$ & $?$ & $?$ & $?$ & ? & $?$ & $?$ & $?$ & $?$ & \\
\hline Globicephala macrorhynchus & 23.6 & $\begin{array}{c}\mathbf{1 0 . 8 7} \\
6.1 \\
\end{array}$ & 0.24 & $\begin{array}{l}6.25 \\
\mathbf{3 . 6} \\
\end{array}$ & & $\begin{array}{l}\mathbf{0 . 5 6} \\
0.4\end{array}$ & & $\begin{array}{c}0.69 \\
\mathbf{0 . 7}\end{array}$ & 1 & 0 & $\begin{array}{l}327 \\
307\end{array}$ \\
\hline G. melas & 21.2 & $\begin{array}{c}\mathbf{8 . 8 6} \\
4.716 \\
\end{array}$ & 0.32 & $\begin{array}{l}3.48 \\
2.82\end{array}$ & & $\begin{array}{l}\mathbf{0 . 7 2} \\
0.71\end{array}$ & & $\begin{array}{l}0.98 \\
\mathbf{1 . 0 1}\end{array}$ & & & $\begin{array}{l}327 \\
305\end{array}$ \\
\hline Grampus griseus & 23.8 & $\begin{array}{c}\mathbf{2 0} \\
13.44 \\
\end{array}$ & 1.90 & $\begin{array}{c}\mathbf{3 . 9} \\
8.83 \\
\end{array}$ & & \begin{tabular}{|l|}
4.9 \\
$\mathbf{0 . 5 3}$ \\
\end{tabular} & & $\begin{array}{l}1>* \\
1.37 \\
\end{array}$ & 1 & 1 & $\begin{array}{l}328 \\
327 \\
\end{array}$ \\
\hline Peponocephala electra & 24.5 & 12.14 & 5.5 & 8.381 & 0.9 & 0.54 & 0.1 & $\begin{array}{l}\mathbf{1 . 0 5} \\
0.04 \\
\end{array}$ & 1 & 1 & $\begin{array}{l}317 \\
329 \\
\end{array}$ \\
\hline Pseudorca crassidens & 18.1 & $\begin{array}{c}8.29 \\
6.1 \\
\end{array}$ & 1.87 & $\begin{array}{c}5.43 \\
4.7 \\
\end{array}$ & & $\begin{array}{l}\mathbf{0 . 5 6} \\
0.4\end{array}$ & & $\begin{array}{c}\mathbf{0 . 7 5} \\
0.5\end{array}$ & 1 & 0 & $\begin{array}{l}327 \\
307\end{array}$ \\
\hline Orcaella brevirostris** & 6.0 & 4.2 & 1.1 & 3.2 & 0.3 & 0.3 & 0.1 & $\sim 1 \leq$ & 1 & 0 & 330 \\
\hline Orcinus orca & $\begin{array}{c}\mathbf{1 8} \\
\\
\\
8.9 \\
16.7 \\
\end{array}$ & $\begin{array}{c}6.61 \\
\\
9.9 \\
\mathbf{1 2 . 6 4} \\
\end{array}$ & $\begin{array}{c}0.05 \\
2.4\end{array}$ & \begin{tabular}{c|}
$\mathbf{1 . 5}$ \\
4.27 \\
\\
\\
5.4 \\
3.36 \\
\end{tabular} & 18.3 & $\begin{array}{l}\mathbf{1 . 8} \\
1.11\end{array}$ & 0.06 & $\begin{array}{c}1> \\
21.14\end{array}$ & 1 & 3 & $\begin{array}{l}331 \\
332 \\
333 \\
334 \\
335 \\
\end{array}$ \\
\hline
\end{tabular}

**In this paper these species are still treated as one single species (with two ecotypes: riverine and marine), however there is recent evidence that each may be a separate species (see details in references 115 and 116) 


\section{APPENDIX 8}
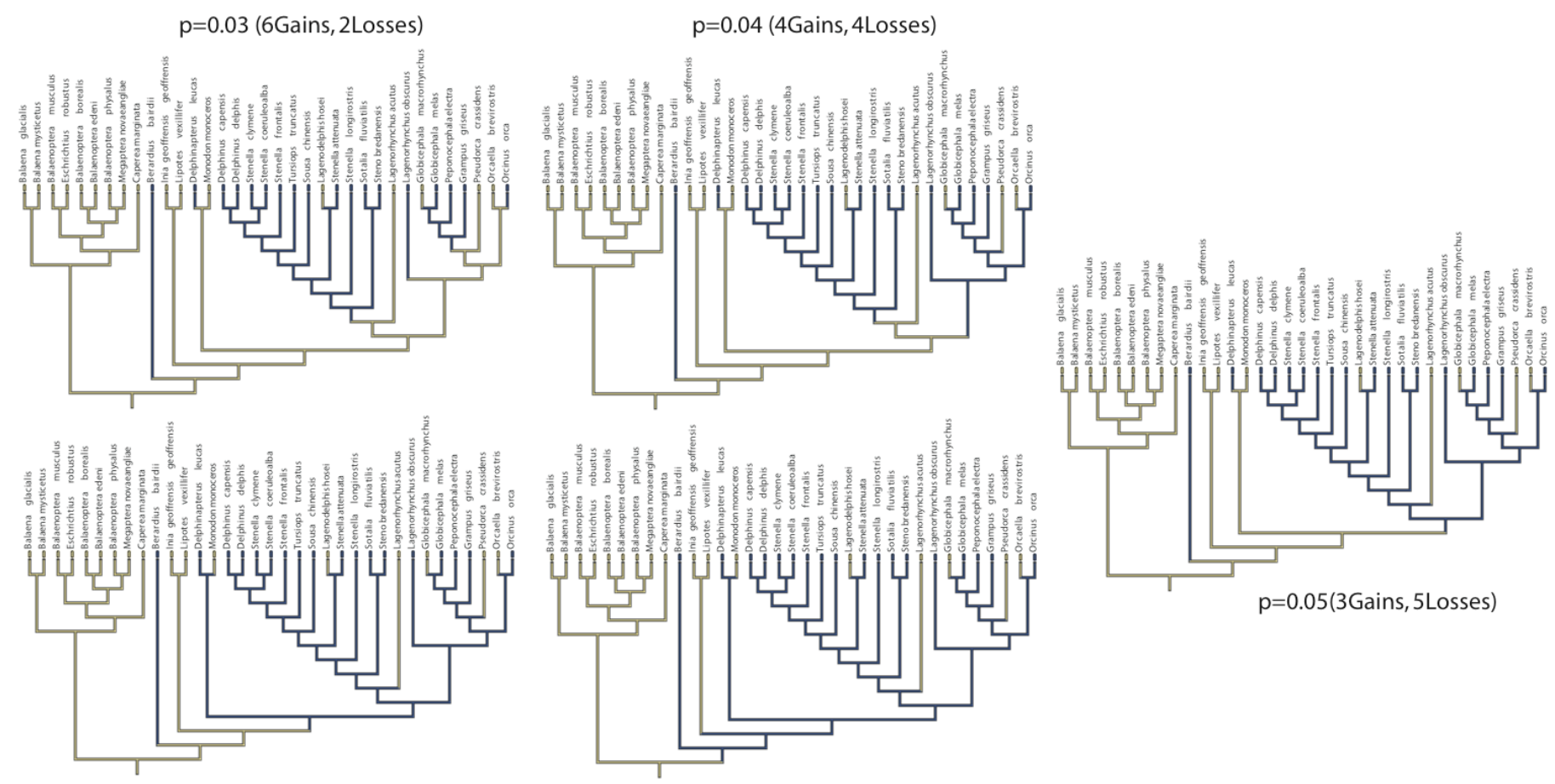

$\mathrm{p}=0.03(2$ Gains, 6 Losses $)$

$\mathrm{p}=0.14(1$ Gain, 7Losses)

Optimization of tonal sound complexity and the association between sociality and tonal sound complexity. Most parsimonious optimizations of tonal sound complexity (based on mean number of inflection points, MIP) and results from the concentrated changes test for sociality and tonal sound complexity (yellow $=$ state 0 , tonal sounds with $\mathrm{MIP} \leq 1$, blue=state 1 , tonal sounds with MIP>1). 
APPENDIX 9. Association between components of sociality and tonal sound complexity. This table summarizes results from SIMMAP analyses of character associations between social components (selecting the highest social state for polymorphic species) and components of tonal sound complexity on the preferred phylogeny.

\begin{tabular}{|c|c|c|c|c|}
\hline \multirow{2}{*}{$\begin{array}{c}\text { TONAL SOUND COMPLEXITY } \\
\text { FOUR STATES }\end{array}$} & \multicolumn{4}{|c|}{ GROUP SIZE $(\mathrm{P}<0.027, \mathrm{P}>0.973)$} \\
\hline & 0 & 1 & 2 & 3 \\
\hline $\begin{array}{c}0(0-1) D i j \\
p \text {-value }\end{array}$ & $\begin{array}{c}0.079 \\
0.91\end{array}$ & $\begin{array}{c}0.0090 \\
0.86\end{array}$ & $\begin{array}{l}-0.028 \\
0.001 *\end{array}$ & \\
\hline $\begin{array}{c}1(1.1-2) D i j \\
\text { p-value }\end{array}$ & $\begin{array}{l}-0.036 \\
0.005^{*}\end{array}$ & $\begin{array}{c}0.0023 \\
0.79\end{array}$ & $\begin{array}{c}0.123 \\
0.92\end{array}$ & \\
\hline $\begin{array}{c}2(2.1-3) \text { Dij } \\
\text { p-value }\end{array}$ & $\begin{array}{c}-0.0049 \\
0.015 *\end{array}$ & $\begin{array}{c}0.052 \\
0.90\end{array}$ & $\begin{array}{l}-0.022 \\
0.007 *\end{array}$ & \\
\hline $\begin{array}{c}3(>3.1) D i j \\
\text { p-value }\end{array}$ & $\begin{array}{c}-0.0051 \\
0.021 * \\
\end{array}$ & $\begin{array}{c}0.026 \\
0.89 \\
\end{array}$ & $\begin{array}{c}-0.0025 \\
0.013^{*}\end{array}$ & \\
\hline TONAL SOUND COMPLEXITY & \multicolumn{4}{|c|}{ GROUP ASSOCIATIONS/STABILITY $(\mathrm{P}<0.027, \mathrm{P}>0.973)$} \\
\hline FOUR STATES & 0 & 1 & 2 & 3 \\
\hline $\begin{array}{c}0(0-1) D i j \\
\text { p-value }\end{array}$ & $\begin{array}{c}0.080 \\
0.93\end{array}$ & $\begin{array}{c}0.0098 \\
0.88\end{array}$ & $\begin{array}{c}-0.031 \\
\mathrm{p}<0.0001 *\end{array}$ & $\begin{array}{c}0.0012 \\
0.84\end{array}$ \\
\hline $\begin{array}{l}1(1.1-2) \text { Dij } \\
\text { p-value }\end{array}$ & $\begin{array}{l}-0.036 \\
0.005 *\end{array}$ & $\begin{array}{c}0.023 \\
0.89\end{array}$ & $\begin{array}{c}0.061 \\
0.91\end{array}$ & $\begin{array}{c}0.042 \\
0.89\end{array}$ \\
\hline $\begin{array}{c}2(2.1-3) \text { Dij } \\
\text { p-value }\end{array}$ & $\begin{array}{l}-0.062 \\
0.009 *\end{array}$ & $\begin{array}{c}0.014 \\
0.92\end{array}$ & $\begin{array}{c}0.023 \\
0.92\end{array}$ & $\begin{array}{l}-0.054 \\
0.010 *\end{array}$ \\
\hline $\begin{array}{c}3(>3.1) D i j \\
\text { p-value }\end{array}$ & $\begin{array}{c}-0.0057 \\
0.009 *\end{array}$ & $\begin{array}{c}0.020 \\
0.94\end{array}$ & $\begin{array}{c}0.0054 \\
0.87\end{array}$ & $\begin{array}{c}-0.00069 \\
0.027 *\end{array}$ \\
\hline TONAL SOUND COMPLEXITY & \multicolumn{4}{|c|}{ GROUP COMPOSITION $(\mathrm{P}<0.027, \mathrm{P}>0.973)$} \\
\hline FOUR STATES & 0 & 1 & 2 & 3 \\
\hline $\begin{array}{c}0(0-1) D i j \\
p \text {-value }\end{array}$ & $\begin{array}{c}0.087 \\
0.94\end{array}$ & $\begin{array}{l}-0.024 \\
0.004 *\end{array}$ & $\begin{array}{c}-0.0069 \\
0.021 *\end{array}$ & $\begin{array}{c}0.0049 \\
0.86\end{array}$ \\
\hline $\begin{array}{c}1 \text { (1.1-2) Dij } \\
\text { p-value }\end{array}$ & $\begin{array}{l}-0.017 \\
0.004 * \\
\end{array}$ & $\begin{array}{c}0.075 \\
0.93 \\
\end{array}$ & $\begin{array}{c}0.031 \\
0.91 \\
\end{array}$ & $\begin{array}{c}-0.0007 \\
0.04 \\
\end{array}$ \\
\hline $\begin{array}{c}2(2.1-3) D i j \\
p \text {-value }\end{array}$ & $\begin{array}{c}-0.0014 \\
0.033\end{array}$ & $\begin{array}{c}-0.0043 \\
0.021 *\end{array}$ & $\begin{array}{c}0.029 \\
0.91\end{array}$ & $\begin{array}{c}0.003 \\
0.84\end{array}$ \\
\hline $\begin{array}{c}3(>3.1) D i j \\
p \text {-value }\end{array}$ & $\begin{array}{c}0.0089 \\
0.84\end{array}$ & $\begin{array}{c}0.014 \\
0.90\end{array}$ & $\begin{array}{c}-0.0016 \\
0.015^{*}\end{array}$ & $\begin{array}{c}0.0055 \\
0.56\end{array}$ \\
\hline \multicolumn{5}{|c|}{ *Significant negative associations, ${ }^{* *}$ significant positive associations } \\
\hline
\end{tabular}


APPENDIX 10. Association between sociality and tonal sound complexity. This table summarizes results from SIMMAP analyses of character associations between social structure (categorized as 1-4) and tonal sound complexity on the preferred phylogeny across reference phylogenies (see Methods)

\begin{tabular}{|c|c|c|c|c|}
\hline $\begin{array}{l}\text { TONAL SOUND } \\
\text { COMPLEXITY }\end{array}$ & \multicolumn{4}{|c|}{$\begin{array}{l}\text { SOCIAL STRUCTURE }(\mathrm{P}<0.027, \mathrm{P}>0.973) \\
\text { Arnasson } \boldsymbol{e} \text { t al. } \mathbf{2 0 0 4} \text { filtered }(\mathbf{n}=\mathbf{3 2 5})\end{array}$} \\
\hline $\begin{array}{c}\text { FOUR STATES } \\
\mathrm{D}=0.375 \mathrm{p}<0.001 * \\
(2 \text { states }) \\
\mathrm{D}=0.356 \mathrm{p}<0.001 * \\
\quad(4 \text { states })\end{array}$ & 0 & 1 & 2 & 3 \\
\hline $\begin{array}{c}0(\leq 1 \text { mean inflection } \\
\text { point }) \\
D \text {-statistic } \\
p \text {-value }\end{array}$ & $\begin{array}{c}0.081 \\
0.73\end{array}$ & $\begin{array}{c}0.053 \\
0.75\end{array}$ & $\begin{array}{l}-0.041 \\
0.003 *\end{array}$ & $\begin{array}{r}-0.0052 \\
0.006^{*}\end{array}$ \\
\hline $\begin{array}{c}1 \text { ( } \leq 1 \text { mean inflection } \\
\text { point }) \\
D \text {-statistic } \\
p \text {-value }\end{array}$ & $\begin{array}{l}-0.044 \\
\mathrm{p}<0.001^{*}\end{array}$ & $\begin{array}{c}0.002 \\
0.95\end{array}$ & $\begin{array}{c}0.111 \\
0.99 * *\end{array}$ & $\begin{array}{c}0.036 \\
0.98 * *\end{array}$ \\
\hline $\begin{array}{c}0(0-1) D i j \\
p \text {-value }\end{array}$ & $\begin{array}{c}0.082 \\
0.94\end{array}$ & $\begin{array}{c}0.016 \\
0.84\end{array}$ & $\begin{array}{l}-0.033 \\
\mathrm{p}<0.001 *\end{array}$ & $\begin{array}{c}0.0094 \\
0.90\end{array}$ \\
\hline $\begin{array}{l}1 \text { (1.1-2) Dij } \\
\text { p-value }\end{array}$ & $\begin{array}{l}-0.037 \\
0.006^{*}\end{array}$ & $\begin{array}{c}0.026 \\
0.91 \\
\end{array}$ & $\begin{array}{c}0.077 \\
0.95 \\
\end{array}$ & $\begin{array}{c}0.022 \\
0.93 \\
\end{array}$ \\
\hline $\begin{array}{l}2(2.1-3) \text { Dij } \\
\text { p-value }\end{array}$ & $\begin{array}{l}-0.0036 \\
0.009 *\end{array}$ & $\begin{array}{c}0.010 \\
0.87 \\
\end{array}$ & $\begin{array}{c}0.020 \\
0.92 \\
\end{array}$ & $\begin{array}{c}-0.0024 \\
0.006 *\end{array}$ \\
\hline $\begin{array}{c}3(>3.1) \text { Dij } \\
\text { p-value }\end{array}$ & $\begin{array}{c}-0.0045 \\
0.006^{*}\end{array}$ & $\begin{array}{c}0.016 \\
0.88 \\
\end{array}$ & $\begin{array}{c}0.005 \\
0.84 \\
\end{array}$ & $\begin{array}{c}0.0025 \\
0.86 \\
\end{array}$ \\
\hline TONAL SOUND & \multicolumn{4}{|c|}{ Nikaido et al. 2001 filtered $(n=341)$} \\
\hline $\begin{array}{c}\text { COMPLEXITY } \\
\text { FOUR STATES } \\
\mathrm{D}=0.382 \mathrm{p}<0.001 * \\
(2 \text { states }) \\
\mathrm{D}=0.358 \mathrm{p}<0.001 * \\
(4 \text { states })\end{array}$ & 0 & 1 & 2 & 3 \\
\hline $\begin{array}{c}0(\leq 1 \text { mean inflection } \\
\text { point }) \\
D \text {-statistic } \\
\text { p-value }\end{array}$ & $\begin{array}{c}0.083 \\
0.75\end{array}$ & $\begin{array}{l}0.053 \\
0.72\end{array}$ & $\begin{array}{c}-0.043 \\
\mathrm{p}<0.001 *\end{array}$ & $\begin{array}{l}-0.0047 \\
0.003^{*}\end{array}$ \\
\hline $\begin{array}{c}1 \text { ( } \leq 1 \text { mean inflection } \\
\text { point }) \\
D \text {-statistic } \\
\text { p-value } \\
\end{array}$ & $\begin{array}{c}-0.046 \\
\mathrm{p}<0.001^{*}\end{array}$ & $\begin{array}{c}0.0004 \\
0.91\end{array}$ & $\begin{array}{l}0.1156 \\
0.99 * *\end{array}$ & $\begin{array}{c}0.036 \\
0.99 * *\end{array}$ \\
\hline $\begin{array}{c}0(0-1) D i j \\
p \text {-value }\end{array}$ & $\begin{array}{c}0.082 \\
0.91 \\
\end{array}$ & $\begin{array}{c}0.00006 \\
0.74 \\
\end{array}$ & $\begin{array}{l}-0.033 \\
0.005 *\end{array}$ & $\begin{array}{c}0.0090 \\
0.85 \\
\end{array}$ \\
\hline $\begin{array}{c}1 \text { (1.1-2) Dij } \\
\text { p-value }\end{array}$ & $\begin{array}{c}-0.037 \\
\mathrm{p}<0.001 *\end{array}$ & $\begin{array}{c}0.0234 \\
0.91\end{array}$ & $\begin{array}{c}0.0776 \\
0.92 \\
\end{array}$ & $\begin{array}{c}0.0235 \\
0.92\end{array}$ \\
\hline $\begin{array}{c}2(2.1-3) \text { Dij } \\
\text { p-value }\end{array}$ & $\begin{array}{c}-0.0044 \\
0.005^{*}\end{array}$ & $\begin{array}{c}0.0133 \\
0.92\end{array}$ & $\begin{array}{c}0.0223 \\
0.87\end{array}$ & $\begin{array}{l}-0.0032 \\
0.020 *\end{array}$ \\
\hline $\begin{array}{c}3(>3.1) D i j \\
p \text {-value }\end{array}$ & $\begin{array}{c}-0.0050 \\
0.011 *\end{array}$ & $\begin{array}{c}0.0165 \\
0.93\end{array}$ & $\begin{array}{c}0.0054 \\
0.89 \\
\end{array}$ & $\begin{array}{c}0.0026 \\
0.90\end{array}$ \\
\hline
\end{tabular}




\begin{tabular}{|c|c|c|c|c|}
\hline \multirow{2}{*}{$\begin{array}{c}\text { TONAL Sound } \\
\text { COMPLEXITY } \\
\text { Four STATES } \\
\mathrm{D}=0.442 / 0.269 \mathrm{p}<0.001 * \\
(2 \text { states }) \\
\mathrm{D}=0.360 / 0.217 \mathrm{p}<0.001 * \\
(4 \text { states })\end{array}$} & \multicolumn{4}{|c|}{$\begin{array}{c}\text { Messenger and McGuire } 1998(n=4) \text { filtered/ } \\
\text { Bayesian }(n=2001)\end{array}$} \\
\hline & 0 & 1 & 2 & 3 \\
\hline $\begin{array}{c}0(\leq 1 \text { mean inflection } \\
\text { point }) \\
\text { D-statistic } \\
\text { p-value }\end{array}$ & $\begin{array}{c}0.085 / 0.072 \\
\mathrm{p}>0.999 * * / 0.86\end{array}$ & $\begin{array}{l}0.064 / 0.016 \\
0.5 / 0.65\end{array}$ & $\begin{array}{c}-0.050 /-0.027 \\
\mathrm{p}<0.001 * / 0.004 *\end{array}$ & $\begin{array}{c}-0.015 /-0.007 \\
\mathrm{p}<0.001 * / 0.025^{*}\end{array}$ \\
\hline $\begin{array}{c}1(\leq 1 \text { mean inflection } \\
\text { point }) \\
D \text {-statistic } \\
p \text {-value }\end{array}$ & $\begin{array}{c}-0.044 /-0.053 \\
\mathrm{p}<0.001 * / 0.004 *\end{array}$ & $\begin{array}{c}-0.015 / 0.015 \\
\mathrm{p}<0.001 * / 0.96\end{array}$ & $\begin{array}{c}0.12 / 0.048 \\
\mathrm{p}>0.999 * * / 0.98 * *\end{array}$ & $\begin{array}{c}0.05 / 0.031 \\
\mathrm{p}>0.999 * * / 0.95\end{array}$ \\
\hline $\begin{array}{c}0(0-1) D i j \\
p \text {-value }\end{array}$ & $\begin{array}{c}0.081 / 0.052 \\
\mathrm{p}>0.999 * * / 0.94\end{array}$ & $\begin{array}{c}0.0062 / 0.013 \\
0.75 / 0.85\end{array}$ & $\begin{array}{l}-0.032 /-0.014 \\
\mathrm{p}<0.001 * / 0.04\end{array}$ & $\begin{array}{l}-0.026 /-0.009 \\
\mathrm{p}<0.001 * / 0.06\end{array}$ \\
\hline $\begin{array}{l}1(1.1-2) D i j \\
\text { p-value }\end{array}$ & $\begin{array}{c}-0.036 /-0.033 \\
\mathrm{p}<0.001 * / 0.021 *\end{array}$ & $\begin{array}{c}0.023 / 0.0007 \\
\mathrm{p}>0.999 * * / 0.81\end{array}$ & $\begin{array}{c}0.072 / 0.037 \\
\mathrm{p}>0.999 * * / 0.93\end{array}$ & $\begin{array}{c}0.038 / 0.025 \\
\mathrm{p}>0.999 * * / 0.92\end{array}$ \\
\hline $\begin{array}{c}2(2.1-3) D i j \\
p \text {-value }\end{array}$ & $\begin{array}{l}-0.0041 / 0.0011 \\
\mathrm{p}<0.001 * / 0.78\end{array}$ & $\begin{array}{c}0.0060 / 0.012 \\
0.75 / 0.87\end{array}$ & $\begin{array}{c}0.026 /-0.0032 \\
0.75 / 0.064\end{array}$ & $\begin{array}{l}-0.0032 / 0.003 \\
\mathrm{p}<0.001 * / 0.81\end{array}$ \\
\hline $\begin{array}{c}3(>3.1) \text { Dij } \\
\text { p-value }\end{array}$ & $\begin{array}{c}-0.0057 /-0.0019 \\
\mathrm{p}<0.001 * / 0.09\end{array}$ & $\begin{array}{c}0.018 / 0.006 \\
0.5 / 0.85\end{array}$ & $\begin{array}{c}0.0028 / 0.0015 \\
0.75 / 0.77\end{array}$ & $\begin{array}{c}0.0037 / 0.005 \\
\mathrm{p}>0.999^{*} * / 0.87\end{array}$ \\
\hline $\begin{array}{l}\text { TONAL SOUND } \\
\text { COMPLEXITY }\end{array}$ & \multicolumn{4}{|c|}{ May-Collado et al. 2007 filtered $(n=1069)$} \\
\hline $\begin{array}{c}\text { FouR STATES } \\
\mathrm{D}=0.378 \mathrm{p}<0.001^{*} \\
\quad(2 \text { states }) \\
\mathrm{D}=0.355 \mathrm{p}<0.001 * \\
\quad(4 \text { states })\end{array}$ & 0 & 1 & 2 & 3 \\
\hline $\begin{array}{c}0(\leq 1 \text { mean inflection } \\
\text { point }) \\
D \text {-statistic } \\
\text { p-value } \\
\end{array}$ & $\begin{array}{c}0.082 \\
0.74\end{array}$ & $\begin{array}{c}0.053 \\
0.73\end{array}$ & $\begin{array}{l}-0.041 \\
0.007 *\end{array}$ & $\begin{array}{c}-0.006 \\
\mathrm{p}<0.001 *\end{array}$ \\
\hline $\begin{array}{c}\text { ( } \leq 1 \text { mean inflection } \\
\text { point }) \\
D \text {-statistic } \\
\text { p-value } \\
\end{array}$ & $\begin{aligned} & -0.045 \\
\mathrm{p} & <0.001 *\end{aligned}$ & $\begin{array}{c}0.0012 \\
0.92\end{array}$ & $\begin{array}{c}0.112 \\
0.99 * *\end{array}$ & $\begin{array}{c}0.037 \\
0.97 * *\end{array}$ \\
\hline $\begin{array}{c}0(0-1) D i j \\
p \text {-value }\end{array}$ & $\begin{array}{c}0.082 \\
0.92\end{array}$ & $\begin{array}{c}0.0018 \\
0.83\end{array}$ & $\begin{array}{c}-0.033 \\
\mathrm{p}<0.001 *\end{array}$ & $\begin{array}{c}0.009 \\
0.89\end{array}$ \\
\hline $\begin{array}{c}1(1.1-2) D i j \\
p \text {-value }\end{array}$ & $\begin{array}{c}-0.037 \\
0.0009 *\end{array}$ & $\begin{array}{c}0.025 \\
0.93 \\
\end{array}$ & $\begin{array}{c}0.077 \\
0.93\end{array}$ & $\begin{array}{c}0.023 \\
0.90\end{array}$ \\
\hline $\begin{array}{c}2(2.1-3) D i j \\
\text { p-value }\end{array}$ & $\begin{array}{c}-0.0037 \\
0.021 *\end{array}$ & $\begin{array}{c}0.013 \\
0.91 \\
\end{array}$ & $\begin{array}{c}0.020 \\
0.90 \\
\end{array}$ & $\begin{array}{l}-0.003 \\
0.012 * \\
\end{array}$ \\
\hline $\begin{array}{c}3(>3.1) D i j \\
\text { p-value }\end{array}$ & $\begin{array}{c}-0.0043 \\
0.013 *\end{array}$ & $\begin{array}{c}0.014 \\
0.89 \\
\end{array}$ & $\begin{array}{c}0.007 \\
0.89 \\
\end{array}$ & $\begin{array}{c}0.002 \\
0.84 \\
\end{array}$ \\
\hline
\end{tabular}

*Significant negative associations, ${ }^{* *}$ significant positive associations 
APPENDIX 11. Regression between group size and tonal sound characteristics. This table summarizes results from PDAP regressionbetween group size and mean minimum frequency (MMinF) and mean number of inflection points (IP) across reference phylogenies (see Methods)

\begin{tabular}{|c|c|c|c|c|c|c|c|}
\hline $\begin{array}{l}\text { ACOUSTIC } \\
\text { PARAMETERS } \\
\text { VS } \\
\text { GROUP SIZE }\end{array}$ & $\begin{array}{l}\text { MAY- } \\
\text { AGNARSSON } \\
2000 \text { TREES } \\
\text { BURNIN }\end{array}$ & $\begin{array}{l}\text { MAY- } \\
\text { COLLADO } \\
\text { FILTERED }\end{array}$ & $\begin{array}{l}\text { MESSENGER } \\
\text { AND } \\
\text { MCGUIRE } \\
(1998) \\
\text { FILTERED } \\
\end{array}$ & $\begin{array}{l}\text { MESSENGER AND } \\
\text { MCGUIRE (1998) } \\
\text { PARSIMONY ON THEIR } \\
\text { NUCLEAR/MORPHOLOGY } \\
\text { DATA }\end{array}$ & $\begin{array}{l}\text { MESSENGER AND } \\
\text { MCGUIRE (1998) } \\
\text { BAYESIAN ON THEIR } \\
\text { NUCLEAR/MORPHOLOGY } \\
\text { DATA }\end{array}$ & $\begin{array}{l}\text { ARNASSON } \\
\text { ET AL } \\
(2003) \\
\text { FILTERED }\end{array}$ & $\begin{array}{l}\text { NIKAIDO } \\
\text { ET AL. } \\
(2001) \\
\text { FILTERED }\end{array}$ \\
\hline \multicolumn{8}{|c|}{ ALL CETACEANS } \\
\hline $\begin{array}{l}\text { MMINF } \\
\text { R-SQUARE } \\
\text { DF } \\
\text { P-VALUE }\end{array}$ & $\begin{array}{l}3.5 \\
29 \\
0.31\end{array}$ & $\begin{array}{l}3.2 \\
29 \\
0.33\end{array}$ & $\begin{array}{l}1.9 \\
29 \\
0.24\end{array}$ & $\begin{array}{l}2.8 \\
21 \\
0.44\end{array}$ & $\begin{array}{l}2.9 \\
21 \\
0.43\end{array}$ & $\begin{array}{l}3.7 \\
29 \\
0.30\end{array}$ & $\begin{array}{l}3.1 \\
29 \\
0.17\end{array}$ \\
\hline $\begin{array}{l}\text { IP } \\
\text { R-SQUARE } \\
\text { DF } \\
\text { P-VALUE }\end{array}$ & $\begin{array}{l}7.5 \\
33 \\
0.05^{*}\end{array}$ & $\begin{array}{l}7.4 \\
33 \\
0.05^{*}\end{array}$ & $\begin{array}{l}10.2 \\
33 \\
0.03 *\end{array}$ & $\begin{array}{l}<1 \\
22 \\
0.86\end{array}$ & $\begin{array}{l}<1 \\
22 \\
0.85\end{array}$ & $\begin{array}{l}5.1 \\
33 \\
0.09\end{array}$ & $\begin{array}{l}3.7 \\
33 \\
0.13\end{array}$ \\
\hline \multicolumn{8}{|c|}{ TOOTHED-WHALES } \\
\hline $\begin{array}{l}\text { MMINF } \\
\text { R-SQUARE } \\
\text { DF } \\
\text { P-VALUE }\end{array}$ & $\begin{array}{l}13.8 \\
23 \\
0.03 *\end{array}$ & $\begin{array}{l}13.2 \\
23 \\
0.03 *\end{array}$ & $\begin{array}{l}5.8 \\
23 \\
0.12\end{array}$ & $\begin{array}{l}11.4 \\
16 \\
0.08\end{array}$ & $\begin{array}{l}9.7 \\
16 \\
0.21\end{array}$ & $\begin{array}{l}15.4 \\
23 \\
0.03 *\end{array}$ & $\begin{array}{l}13.5 \\
23 \\
0.03 *\end{array}$ \\
\hline $\begin{array}{l}\text { IP } \\
\text { R-SQUARE } \\
\text { DF } \\
\text { P-VALUE }\end{array}$ & $\begin{array}{l}7.3 \\
24 \\
0.09\end{array}$ & $\begin{array}{l}7.1 \\
24 \\
0.09\end{array}$ & $\begin{array}{l}10 \\
24 \\
0.06\end{array}$ & $\begin{array}{l}<1 \\
17 \\
0.92\end{array}$ & $\begin{array}{l}<1 \\
17 \\
0.93\end{array}$ & $\begin{array}{l}4.7 \\
24 \\
0.14\end{array}$ & $\begin{array}{l}3.5 \\
24 \\
0.17\end{array}$ \\
\hline
\end{tabular}

*Significant results 
APPENDIX 12. Regression between duration and other acoustic variables. This table summarizes results from PDAP regression analyses between duration (s) and absolute (AbsMinF) and mean minimum (MMin) frequency and mean number of inflection

\begin{tabular}{|c|c|c|c|c|c|c|c|}
\hline $\begin{array}{l}\text { ACOUSTIC } \\
\text { PARAMETERS } \\
\text { VS } \\
\text { GROUP SIZE }\end{array}$ & $\begin{array}{l}\text { MAY- } \\
\text { AGNARSSON } \\
2000 \text { TREES } \\
\text { BURNIN }\end{array}$ & $\begin{array}{l}\text { MAY- } \\
\text { COLLADO } \\
\text { FILTERED }\end{array}$ & $\begin{array}{l}\text { MESSENGER } \\
\text { AND } \\
\text { MCGUIRE } \\
(1998) \\
\text { FILTERED } \\
\end{array}$ & $\begin{array}{l}\text { MESSENGER AND } \\
\text { MCGUIRE (1998) } \\
\text { PARSIMONY ON THEIR } \\
\text { NUCLEAR/MORPHOLOGY } \\
\text { DATA }\end{array}$ & $\begin{array}{l}\text { MESSENGER AND } \\
\text { MCGUIRE (1998) } \\
\text { BAYESIAN ON THEIR } \\
\text { NUCLEAR/MORPHOLOGY } \\
\text { DATA }\end{array}$ & $\begin{array}{l}\text { ARNASSON } \\
\text { ET AL } \\
(2003) \\
\text { FILTERED }\end{array}$ & $\begin{array}{l}\text { NIKAIDO } \\
\text { ET AL. } \\
(2001) \\
\text { FILTERED }\end{array}$ \\
\hline \multicolumn{8}{|c|}{ AlL CETACEANS } \\
\hline $\begin{array}{l}\text { ABS MINF } \\
\text { R-SQUARE } \\
\text { DF } \\
\text { P-VALUE }\end{array}$ & $\begin{array}{l}17 \\
31 \\
0.017\end{array}$ & $\begin{array}{l}16.5 \\
31 \\
0.018\end{array}$ & $\begin{array}{l}19.4 \\
31 \\
0.01\end{array}$ & $\begin{array}{l}29.7 \\
20 \\
0.008\end{array}$ & $\begin{array}{l}37 \\
20 \\
0.002\end{array}$ & $\begin{array}{l}16.5 \\
31 \\
0.02\end{array}$ & $\begin{array}{l}16.6 \\
31 \\
0.018\end{array}$ \\
\hline $\begin{array}{l}\text { MMINF } \\
\text { R-SQUARE } \\
\text { DF } \\
\text { P-VALUE }\end{array}$ & $\begin{array}{l}17.5 \\
29 \\
0.019\end{array}$ & $\begin{array}{l}19 \\
29 \\
0.014\end{array}$ & $\begin{array}{l}12.5 \\
29 \\
0.05\end{array}$ & Not significant & Not significant & $\begin{array}{l}19 \\
29 \\
0.014\end{array}$ & $\begin{array}{l}19 \\
29 \\
0.014\end{array}$ \\
\hline $\begin{array}{l}\text { IP } \\
\text { R-SQUARE } \\
\text { DF } \\
\text { P-VALUE }\end{array}$ & $\begin{array}{l}11.9 \\
32 \\
0.05\end{array}$ & $\begin{array}{l}11.7 \\
32 \\
0.04\end{array}$ & $\begin{array}{l}9.4 \\
32 \\
0.07\end{array}$ & $\begin{array}{l}35.6 \\
21 \\
0.002\end{array}$ & $\begin{array}{l}42.3 \\
20 \\
0.001\end{array}$ & $\begin{array}{l}11.9 \\
32 \\
0.04\end{array}$ & $\begin{array}{l}12.2 \\
32 \\
0.04\end{array}$ \\
\hline \multicolumn{8}{|c|}{ TOOTHED-WHALES } \\
\hline $\begin{array}{l}\text { ABS MINF } \\
\text { R-SQUARE } \\
\text { DF } \\
\text { P-VALUE }\end{array}$ & $\begin{array}{l}37.8 \\
22 \\
0.001\end{array}$ & $\begin{array}{l}39 \\
22 \\
0.001\end{array}$ & $\begin{array}{l}38.7 \\
22 \\
0.001\end{array}$ & $\begin{array}{l}43.9 \\
15 \\
0.003\end{array}$ & $\begin{array}{l}45.9 \\
15 \\
0.002\end{array}$ & $\begin{array}{l}39.2 \\
22 \\
0.001\end{array}$ & $\begin{array}{l}38.1 \\
22 \\
0.001\end{array}$ \\
\hline $\begin{array}{l}\text { MMINF } \\
\text { R-SQUARE } \\
\text { DF } \\
\text { P-VALUE }\end{array}$ & $\begin{array}{l}23.8 \\
23 \\
0.013\end{array}$ & $\begin{array}{l}26.7 \\
23 \\
0.008\end{array}$ & $\begin{array}{l}24.6 \\
23 \\
0.01\end{array}$ & $\begin{array}{l}25.8 \\
16 \\
0.03\end{array}$ & $\begin{array}{l}23.9 \\
16 \\
0.03\end{array}$ & $\begin{array}{l}26.4 \\
23 \\
0.008\end{array}$ & $\begin{array}{l}26 \\
23 \\
0.009\end{array}$ \\
\hline $\begin{array}{l}\text { IP } \\
\text { R-SQUARE } \\
\text { DF } \\
\text { P-VALUE }\end{array}$ & $\begin{array}{l}44.8 \\
23 \\
p<0.001\end{array}$ & $\begin{array}{l}44.1 \\
23 \\
\mathrm{P}<0.001\end{array}$ & $\begin{array}{l}44.2 \\
23 \\
p<0.001\end{array}$ & $\begin{array}{l}38.3 \\
16 \\
0.006\end{array}$ & $\begin{array}{l}46 \\
15 \\
0.002\end{array}$ & $\begin{array}{l}45 \\
23 \\
p<0.001\end{array}$ & $\begin{array}{l}46.1 \\
23 \\
p<0.001\end{array}$ \\
\hline
\end{tabular}


APPENDIX 13

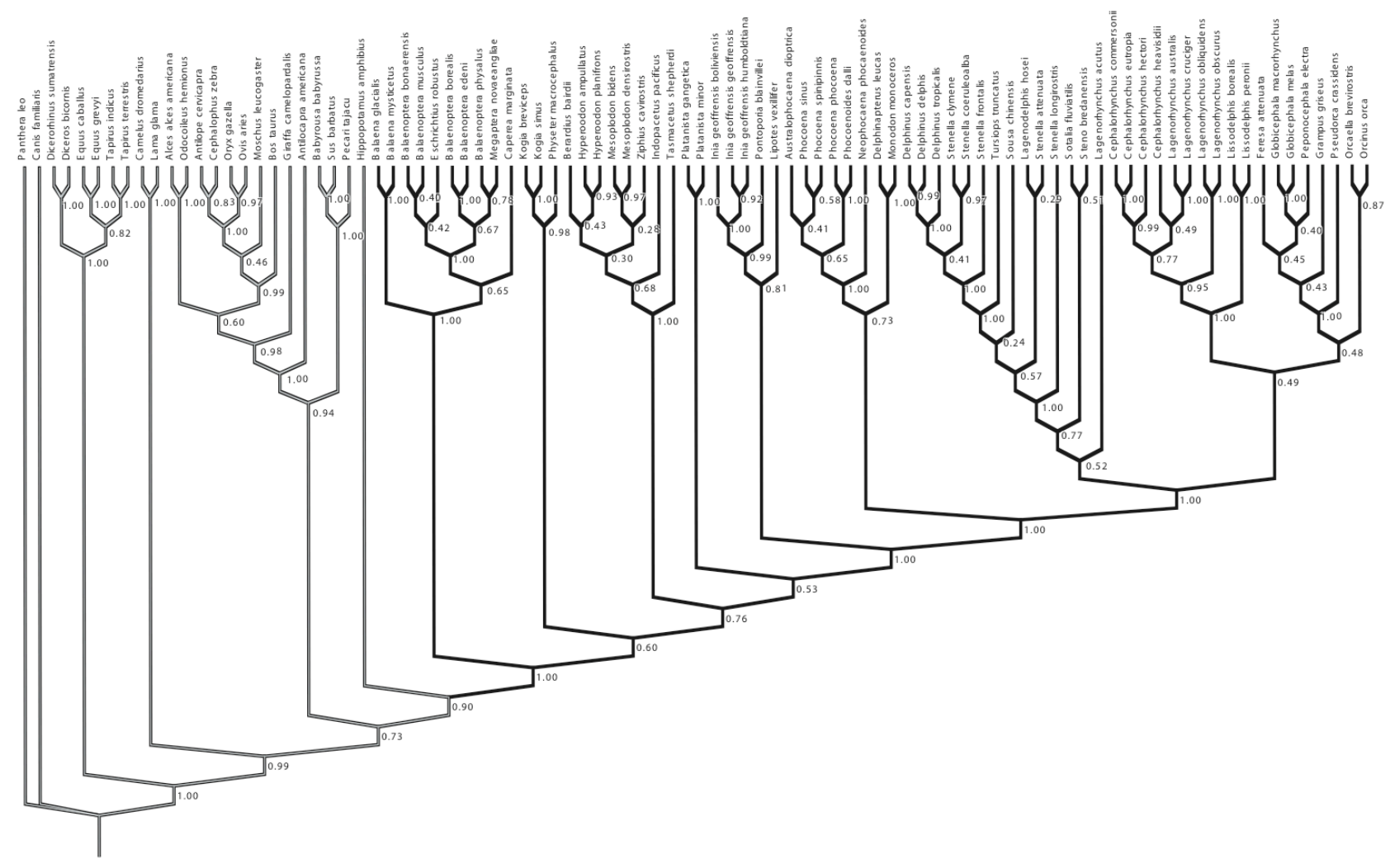

Phylogeny of Cetacea. This figure reproduces the preferred phylogenetic hypothesis of May-Collado et al. (2007), used here for all main analyses. Numbers on nodes represent posterior probabilities 
1. Best PB, Schaeff CM, Reeb D, Palsboll PJ: Composition and possible function of social groupings of southern right whales in South African waters. Behaviour 2003, 140:1469-1494.

2. Braham HC, Rice DW: The Right whale Balaena glacialis. Marine Fisheries Review 1984, 46: 38-47.

3. Bannister LJ: 2002. Baleen Whales (Mysticetes). In: Encyclopedia of Marine Mammals Edited by Perrin WF, Wursig B, Thewissen JGM. San Diego: Academic Press; 2002: 62-73.

4. Richardson WJ, Finley KJ, Miller GW, Davis RA, Koski WR: Feeding social and migration behavior of bowhead whales, Balaena mysticetus, in Baffin Bay vs the Beaufort Sea regions with different amounts of human activity. Marine Mammal Science 1995, 11: 1-45.

5. Landino SW, Treacy SD, Zerwick SA, Dunlap JB: A large aggregation of bowhead whales (Balaena mysticetus) feeding near Point Barrow, Alaska in late October 1992. Arctic 1994, 47: 232-235.

6. Schilling MR, Seipt I, Weinrich MT, Frohock SE, Kuhlberg AE, Clapham PJ: Behavior of individually-identified sei whales Balaenoptera borealis during an episodic influx into the southern Gulf of Maine in 1986. Fishery Bulletin 1992, 90: 749-755.

7. Lucena A: Estrutura populacional da Balaenoptera bonaerensis (Burmeister) (Cetacea, Balaenopteridae) nas áreas de reprodução do Oceano Atlântico Sul. Revista Brasileira de Zoology 2006, 23:176-185.

8. O'Callaghan TM, Baker CS: Summer cetacean community, with particular reference to Bryde's whales in the Hauraki Gulf, New Zealand. Doc Science Internal Series 55. Wellington, New Zealand: Department of Conservation Press; 2002:18 pp.

9. Tershy BR: Body Size, Diet, Habitat Use, and Social Behavior of Balaenoptera Whales in the Gulf of California. Journal of Mammalogy 1992, 73: 477-486.

10. Berube $M$, Berchok $C$, Sears R: Observation of a male-biased sex ratio in the Gulf of St. Lawrence fin whales (Balaenoptera physalus): temporal, geographical, or group structure segragation? Marine Mammal Science 2001, 17:371-381.

11. Canese S, Cardinali A, Fortuna CM, Giusti M, Lauriano G, Salvati E, Greco S: The first identified winter feeding ground of fin whales (Balaenoptera physalus) in the Mediterranean Sea. Journal of Marine Biological Association of the United Kingdom 2006, 86: 903-907. 
12. Gannier A: Summer distribution of fin whales (Balaenoptera physalus) in the Northwestern Mediterranean Marine Mammals Sanctuary. Revue D Ecologie La Terre et la Vie 2002, 57:135-150.

13. Panigada S, Di Sciara GN, Panigada MZ: Fin whales summering in the Pelagos Sanctuary (Mediterranean Sea): Overview of studies on habitat use and diving behaviour. Chemistry and Ecology 2006, 22: 255-263.

14. Tyack $\mathrm{P}$, Whitehead $\mathrm{H}$ : Male competition in large groups of wintering humpback whales. Behaviour 1982, 83: 1-23.

15. Mobley JR, Herman LM: Transience of social affiliation among humpback whales (Megaptera novaeangliae) on the Hawaiian wintering grounds. Canadian Journal of Zoology 1985. 63:762-772.

16. Weinrich MT: Stable social association among humpback whales (Megaptera novaeangliae) in the southern Gulf of Main. Canadian Journal of Zoology 1991, 69: 3012-3019.

17. Weinrich MT, Rosenbaum H, Baker CS, Blackmer AL, Whitehead H: The influence of maternal lineages on social affiliations among humpback whales (Megaptera novaeangliae) on their feeding grounds in the Southern Gulf of Main. Journal of Heredity 2006, 97: 226-234.

18. Valsecchi E, Hale $\mathrm{P}$, Corkeron $\mathrm{P}$, Amoss W: Social structure in migrating humpback whales (Megaptera novaeangliae). Molecular Ecology 2002, 11: 507-518.

19. Clapham PJ, Palsboll PJ, Mattila DK, Vasquez O: Composition and dynamics of humpback whales competitive groups in the West-Indies. Behaviour 1992, 122:182194.

20. Brown MB, Corkeron PJ, Hale PJ, Schultz KW, Bryden MM: Evidence for a SexSegregated Migration in the Humpback Whale (Megaptera novaeangliae). Proceedings Biological Sciences 1995, 259: 229-234.

21. Straley JM, Herman LM, Jacobsen J: Evidence of a feeding aggregation of humpback whales (Megaptera novaeangliae) around Kodiak Island, Alaska. Marine mammal science 1999, 15: 210-220.

22. Pomilla $\mathrm{C}$, Rosenbaum $\mathrm{HC}$ : Estimates of relatedness in groups of humpback whales (Megaptera novaeangliae) on two wintering grounds of the Southern Hemisphere. Molecular Ecology 2006, 15: 2541-2555. 
23. Urban-Ramirez J, Rojas-Bracho L, Perez-Cortes H, Gomez-Gallardo A, Swartz SL, Ludwig S, Brownell RL: A review of gray whales (Eschrichtius robustus) on their wintering grounds in Mexican waters. Journal of Cetacean Research and Management 2003, 5: 281-295.

24. Calambokidis J, Darling JD, Deecke V, Gearin P, Gosho M, Megill W, Tombach $\mathrm{CM}$, Goley D, Toropova C, Gisborn B: Abundance, range, and movements of a feeding aggregation of gray whales (Eschrichtius robustus) from California to Southern Alaska in 1998. Journal of Cetacean Research and Management 2002, 4:267276.

25. Baird RW: Sightings of dwarf (Kogia sima) and Pygmy (K. breviceps) from the main Hawaiian islands. Pacific Science 2005, 59:461-466.

26. Cardona-Maldonado MA, Mignucci-Giannoni AA: Pygmy and dwarf sperm whales in Puerto Rico and the Virgin Islands with a review of Kogia in the Caribbean. Caribbean Journal of Science 1999, 35: 29-37.

27. Acevedo-Guiterrez, A. Group Behavior. In: Encyclopedia of Marine Mammals Edited by Perrin WF, Wursig B, Thewissen JGM. San Diego: Academic Press; 2002: 537-545.

28. May-Collado LJ, Gerrodete T, Calambokidis J, Rassumen K, Sereg I: Distribution of Cetaceans sightings in the EEZ of Costa Rica. Revista de Biología Tropical 2005, 53:249-263.

29. Caldwell DK, Caldwell MC: Pygmy sperm whale Kogia breviceps (de Blainville 1838) and dwarf sperm whale Kogia simus (Owen 1866). In: Handbook of Marine Mammals Edited by Ridgway SH, Harrison Sir H. New York: Academic Press; 1989: 235-260 [vol. 4.].

30. McAlpine DF: Pygm and Dwarf sperm whales (Kogia breviceps and K. sima). In: Encyclopedia of Marine Mammals Edited by Perrin WF, Wursig B, Thewissen JGM. San Diego: Academic Press; 2002: 1007-1009.

31. Nagorsen DW, Stewart GE: A dwarf sperm whale (Kogia simus) from the Pacific coat of Canada. Journal of Mammalogy 1983, 64:505-506.

32. Christal J, Whitehead H, Lettevall E: Sperm whale social units: variation and change. Canadian Journal of Zoology 1998, 76: 1431-1440.

33. Mesnick SL: Genetic relatedness in sperm whales: evidence and cultural implications. Behavioral and Brain Science 2001, 24: 346-347. 
34. Lettevall E, Richter C, Jaquet N, Slooten E, Dawson S, Whitehead H, Christal J, McCall Howard P: Social structure and residency in aggregatins of male sperm whales. Canadian Journal of Zoology 2002, 80: 1189-1196.

35. Rendell LE, Whitehead HL: Do sperm whales share coda vocalizations? Insights into coda usage from acoustic size measurement. Animal Behaviour 2004, 67: 865874.

36. Whitehead H: Baby-sitting, dive synchrony, and indications of alloparental care in sperm whales. Behavioral Ecology and Sociobiology 1996, 38: 237-244.

37. Whitehead H: Sperm whales (Physeter macrocephalus). In: Encyclopedia of Marine Mammals Edited by Perrin WF, Wursig B, Thewissen JGM. San Diego: Academic Press; 2002:1165-1171

38. Whitehead H, Weilgart LS: Patterns of visually observable behaviour and vocalizations in groups of female sperm whales. Behaviour 1991, 118: 275-296.

39. Whitehead H, Weilgart LS: The sperm whale: social females and roving males. In Cetacean societies: field studies of dolphins and whale Edited by Mann J, Connor RC, Tyack PL, Whitehead H. Chicago: University of Chicago Press; 2000: 154-172.

40. Whitehead $\mathrm{H}$, Waters $\mathrm{S}$, Lyrholm T: Social organization in female sperm whales and their offspring: constant companions and casual acquaintances. Behavioral Ecology and Sociobiology 1991, 29: 385-389.

41. Kasuya T: Distribution and behavior of Baird's beake whales off the Pacific coast of Japan. The Scientific Report of Whales Research Institute 1986, 37: 61-83.

42. Kasuya T, Brownell RLJr, Balcomb III KC: Life history of Baird's beaked whales off the Pacific coast of Japan. Reports of the International Whaling Commission 1997, 47: 969-979.

43. Balcomb KC: Baird's beaked whale Berardius baiirdi Stejneger,1883: Arnoux's beaked whale Berardius arnuxii Duvernoy, 1851. In: Handbook of Marine Mammals Edited by Ridgway SH, Harrison Sir H. New York: Academic Press; 1989: 261-288. [vol. 4].

44. Aurioles-Gamboa D: Notes on a mass stranding of Baird beaked-whales in the Gulf of California, Meximo. California Fish and Game 1992, 78:116-123.

45. Connor RC, Mann J, Tyack PL, Whitehead H: Social evolution in toothed whales. Trends in Ecology and Evolution 1998, 13: 228-232.

46. Dawson S, Barlow J, Ljungblad D: Sounds recorded from Baird's beaked whale, Berardius bairdii. Marine Mammal Science 1998, 14: 335-344. 
47. Ponganis PJ, Kooyman GL: Multiple sightings of Arnoux's beaked whales along the Victoria land coast. Marine Mammal Science 1995, 11: 247-250.

48. Rogers TL, Brown SM: Acoustic observations of Arnoux's beaked whale (Berardius arnuxii) off Kemp Land, Antarctica. Marine Mammal Science 1999, 15:192-198.

49. Hooker SK, Whitehead H, Gowans S, Baird RW: Fluctuations in distribution and patterns of individual range use of northern bottlenose whales. Marine Ecology Progress Series 2002, 225:287-297.

50. Gowans S, Rendell L: Head-butting in northern bottlenose whales (Hyperoodon ampullatus): a possible function for big heads? Marine Mammal Science 1999, 15:1342-1350.

51. Gowans S, Whitehead H, Hooker SK: Social organization in northern bottlenose whales, Hyperoodon ampullatus: not driven by deep-water foraging? Animal Behaviour 2001, 62:369-377.

52. Kasamatsu F, Joyce GD: Current status of odontocetes in the Antarctic. Antarctic Science 1995, 7: 365-379.

53. Hooker SK, Baird RW. Observations of Sowerby's Beaked Whales, Mesoplodon bidens, in the Gully, Nova Scotia. Canadian Field-Naturalist 1999, 113: 273-277.

54. Pitman RL: 2002. Mesoplodon Whales (Mesoplodon sp). In: Encyclopedia of Marine Mammals Edited by Perrin WF, Wursig B, Thewissen JGM. San Diego: Academic Press; 2002: 738-742.

55. Mead JG: Beaked whales of the genus Mesoplodon. In: Handbook of Marine Mammals Edited by Ridgway SH, Harrison Sir H. New York: Academic Press; 1989: 349-415. [vol. 4.].

56. Baird RW, McSweeney DJ, Ligon AD, Webster DL: Tagging feasibility and diving of Cuvier's beaked whales (Ziphius cavirostris) and Blainville's beaked whales (Mesoplodon densirostris) in Hawaii. Report to the Wildlife Fund, Volcano, HI. Order No. AB133F-03-SE-0986. SWFSC, NMFS, La Jolla CA 2004.

57. Borsa P: Marine mammal strandings in the New Caledonia region, Southwest Pacific. Comptes Rendus Biologies 2006, 329: 277-288.

58. MacLeod CD, Zuur AF: Habitat utilization by Blainville's beaked whales off Great Abaco, northern Bahamas, in relation to seabed topography. Marine Biology 2005, 147: 1-11. 
59. Baird RW, Webster DL, McSweeney DJ, Ligon AD, Schorr GS: Diving behavior and ecology of Cuvier's (Ziphius cavirostris) and Blainville's beaked whales (Mesoplodon densirostris) in Hawaii. Report to Cascadia Research Collective, WA. Order No. AB133F-04-RQ-0928. SWFSC, NMFS, La Jolla CA 2005.

60. Gomercic H, Gomercic MD, Gomercic T, Lucic H, Dalebout M, Galov A, Skrtic D, Curkovic S, Vukovic S, Huber D: Biological aspects of Cuvier's beaked whale (Ziphius cavirostris) recorded in the Croatian part of the Adriatic Sea. European Journal of Wildlife Research 2006, 52: 182-187

61. Marini L, Consiglio C, Angradi AM, Catalano B, Sanna A, Valentini T:

Distribution, abundance and seasonality of cetaceans sighted during scheduled ferry crossings in the central Tyrrhenian Sea: 1989-1992. Italian Journal of Zoology 1996, 63: $381-388$.

62. Heyning JE: Cuvier's Beaked Whale Ziphius cavirostris G. Cuvier, 1823. In: Handbook of Marine Mammals Edited by Ridgway SH, Harrison Sir H. New York: Academic Press; 1989: 289-308. [vol.4]

63. Akbar M, Mehal QM, Arshed MJ: Population estimation of Indus dolphin from Jinnah-Guddu Barrage. Journal of Applied Sciences 2004, 4:21-23.

64. Kasuya T, Haque AKM: Some informations on distributions and seasonal movement of the Ganges Dolphin. The Scientific Report of Whales Research Institute 1972, 24: 109-115.

65. Reeves RR, Brownell RLJr: Susu, Platanista gangetica (Roxburg 1801) and Platanista minor (Owen 1853). In: Handbook of Marine Mammals Edited by Ridgway SH, Harrison Sir H. New York: Academic Press; 1989: 69-99. [vol.4].

66. Braulik GT: Status assessment of the Indus river dolphin, Platanista gangetica minor, March-April 2001. Biological Conservation 2006, 129:579-590.

67. Smith BD: 1990 Status and conservation of the Ganges river dolphin Platanista gangetica in the Karnali River, Nepal. Biological Conservation 1993, 66:159-169.

68. Smith BD: Susu and Bhulan (Platanista gangetica gangetica and P.g.minor). In: Encyclopedia of Marine Mammals Edited by Perrin WF, Wursig B, Thewissen JGM. San Diego: Academic Press; 2002:1208-1213.

69. Smith BD, Braulik G, Strindberg S, Ahmed B, Mansur R: Abundance of Irrawady dolphins (Orcaella brevirostris) and Ganges River dolphins (Platanista gangetica gangetica) estimated using concurrent counts made by independent teams in waterways of the sundarbans mangrove forest in Bangladesh. Marine Mammal Science 2006, 22: 527-547. 
70. Smith BD, Ahmed B, Ali ME, Braulik G: Status of the Ganges river dolphin or shushuk Platanista gangetica in Kaptai Lake and southern rivers of Bangladesh. Oryx 2001, 35: 61-72.

71. Reeves RR: River dolphins. In: Encyclopedia of Marine Mammals Edited by Perrin WF, Wursig B, Thewissen JGM. San Diego: Academic Press; 2002:1039-1242.

72. Best RC, da Silva VMF: Amazon River Dolphin, Boto Inia geoffrensis (de Blainville 1817). In: Handbook of Marine Mammals Edited by Ridgway SH, Harrison Sir H. New York: Academic Press; 1989: 1-23. [vol.4].

73. Aliaga-Rossel E: Distribution and abundance of the river dolphin (Inia geoffrensis) in the Tijamuchi River, Beni, Bolivia. Aquatic Mammals 2002, 28: 312323.

74. Da Silva VMF: Amazon River Dolphin (Inia geoffrensis). In: Encyclopedia of Marine Mammals Edited by Perrin WF, Wursig B, Thewissen JGM. San Diego: Academic Press; 2002: 18-20.

75. Martin AR, da Silva VMF: Sexual dimorphism and body scarring in the boto (Amazon River dolphin) Inia geoffrensis. Marine Mammals Science 2006, 22: 25-33.

76. McGuire TL, Winemiller KO: Ocurrence patterns, habitat associations, and potential prey of the river dolphin, Inia geoffrensis, in the Cinaruco River, Venezuela. Biotropica 1998, 30: 625-638.

77. Muizon de C: River dolphins evolutionary history. In: Encyclopedia of Marine Mammals Edited by Perrin WF, Wursig B, Thewissen JGM. San Diego: Academic Press; 2002: 1043-1050.

78. Trujillo FG: Habitat use and social behaviour of the freshwater dolphin Inia geoffrensis (de Blainville 1817) in the Amazon and Orinoco basins. Ph.D. Thesis, University of Aberdeen, Scotland 2000: 138 pp.

79. Brownell RLJr: Franciscana, Pontoporia blainvillei (Gervais and d'Orbigny 1844). In: Handbook of Marine Mammals Edited by Ridgway SH, Harrison Sir H. New York: Academic Press; 1989: 45-67. [vol.4].

80. Cremer MJ, Simoes-Lopes PC: The occurrence of Pontoporia blainvillei (Gervais \& d'Orbigny)(Cetacea, Pontoporidae) in an estuarine area in southern Brazil. Revista Brasileira de Zoologia 2005, 22: 717-723.

81. Crespo EA: Franciscana (Pontoporia blainvillei). In: Encyclopedia of Marine Mammals Edited by Perrin WF, Wursig B, Thewissen JGM. San Diego: Academic Press; 2002: $482-484$. 
82. Danilewicz D, Claver JA, Perez Carrera AL, Secchi ER, Fontoura NF: Reproductive biology of male Franciscanas from Rio Grande do Sul, southern Brazil. Fishery Bulletin 2004, 102: 581-592.

83. Kaiya Z: Baiji (Lipotes vexillifer). In: Encyclopedia of Marine Mammals Edited by Perrin WF, Wursig B, Thewissen JGM. San Diego: Academic Press; 2002: 58-61.

84. Pexiun C: Baiji, Lipotes vexillifer (Miller 1918). In: Handbook of Marine Mammals Edited by Ridgway SH, Harrison Sir H. New York: Academic Press; 1989: 25-43. [vol.4].

85. Zhang X, Wang D, Liu R, Wei Z, Hua Y, Wang Y, Chen Z, Wang L: The Yangtze River dolphin or baiji (Lipotes vexillifer): population status and conservation issues in the Yangtze River, China. Aquatic Conservation: Marine and Freshwater Ecosystems 2003, 13: 51-64.

86. Brownell JRJr, Herald ES: Lipotes vexillifer. Mammalian Species 1972, 10: 1-4.

87. Brownell RLJr, Clapham RJ: Spectacle Porpoise Phocoena dioptrica (Lahille, 1912). In: Handbook of Marine Mammals Edited by Ridgway SH, Harrison Sir H. New York: Academic Press; 1999: 379-393. [vol.6].

88. Goodall RNP: Spectacled Porpoise (Phocoena dioptrica). In: Encyclopedia of Marine Mammals Edited by Perrin WF, Wursig B, Thewissen JGM. San Diego: Academic Press; 2002: 1158-1161.

89. Goodall RNP, Schiavini ACM: On the biology of the spectacled porpoise, Australophocoena dioptrica. International Whaling Commision 1995, Special Issue 16: 411-453.

90. Culik BM, Koschinski S, Tregenza N, Ellis GM: Reactions of harbor porpoises Phocoena phocoena and herring Clupea harengus to acoustic alarms. Marine Ecology Progress Series 2001, 211:255-260.

91. Bjфrge A, Tolley KA: Harbor porpoise (Phocoena phocoena). In: Encyclopedia of Marine Mammals Edited by Perrin WF, Wursig B, Thewissen JGM. San Diego: Academic Press; 2002: 549-551.

92. Hoek W: An unusual aggregation of Harbor porpoises (Phocoena phocoena). Marine Mammal Science 1992, 8:152-155.

93. Read, A. J. Harbor porpoise Phocoena phocoena (Linnaeus, 1758). In: Handbook of Marine Mammals Edited by Ridgway SH, Harrison Sir H. New York: Academic Press; 1999: 323-355. [vol.6]. 
94. Read AJ, Westgate AJ: Monitoring the movements of harbour porpoises (Phocoena phocoena) with satellite telemetry. Marine Biology 1997, 130: 315-322.

95. Jaramillo-Legorreta AM, Rojas-Bracho L, Gerrodette T: A new abundance estimate for vaquitas. First step for recovery. Marine Mammal Science 1999, 15: 957-973.

96. Rojas-Brucho L, Jaramillo-Lagorreta A: Vaquita (Phocoena sinus). In:

Encyclopedia of Marine Mammals Edited by Perrin WF, Wursig B, Thewissen JGM. San Diego: Academic Press; 2002: 1277-1280.

97. Vidal O, Brownell RL, Findley LT: Vaquita - Phocoena sinus (Norris and McFarland, 1958). In: Handbook of Marine Mammals Edited by Ridgway SH, Harrison Sir H. New York: Academic Press; 1999: 357-378. [vol.4].

98. Jefferson TA: Phocoenoides dalli. Mammalian Species 1988, 319: 1-7.

99. Jefferson TA: Dall's Porpoise (Phocoenoides dalli). In: Encyclopedia of Marine Mammals Edited by Perrin WF, Wursig B, Thewissen JGM. San Diego: Academic Press; 2002: 308-310.

100. Jefferson TA, Hung SK: Neophocaena phocaenoides. Mammalian Species 2004, 746: $1-12$.

101. Amano M. 2002. Finless Porpoise (Neophocaena phocaenoides). In: Encyclopedia of Marine Mammals Edited by Perrin WF, Wursig B, Thewissen JGM. San Diego: Academic Press; 2002: 432-435.

102. Kasuya T: Neophocaena phocaenoides (Cuvier, 1829). In: Handbook of Marine Mammals Edited by Ridgway SH, Harrison Sir H. New York: Academic Press; 1999: 411-442. [vol.6].

103. Wang K, Wang D, Akamatsu T, Li S, Xiao J: A passive acoustic monitoring method applied to observation and group size estimation of finless porpoises. Journal of the Acoustical Society of America 2005, 118: 1180-1185.

104. Wei ZD, Wang D, Kuang X, Wang K, Wang X, Xiao J, Zhao Q, Zhang X: Observations on behavior and ecology of the Yangtze finless porpoise (Neophocaena phocaenoides asiaeorientalis) group at Tian-e-Zhou Oxbow of the Yangtze River. Raffles Bulletin of Zoology 2002, Suppl 10:97-103.

105. Hay KA, Mansfield AW: Narwhal, Monodon monocerus (Linnaeus 1758). In: Handbook of Marine Mammals Edited by Ridgway SH, Harrison Sir H. New York: Academic Press; 1989: 145-176. [vol.4]. 
106. Heide-Jørgensen MP: Narwhal (Monodon monocerus). In: Encyclopedia of Marine Mammals Edited by Perrin WF, Wursig B, Thewissen JGM. San Diego: Academic Press; 2002: 783-787.

107. Cosens SE, Dueck LP: Group size and activity patterns of belugas (Delphinapterus leucas) and narwhals (Monodon moncerus) during spring migration in Lancaster Sound. Canadian Journal of Zoology 1991, 6:1630-1635.

108. Pallsboll PJ, Heide-Jorgensen MP, Dietz R: Population structure and seasonal movements of narwhals, Monodos monocerus, determined from mtDNA analysis. Heredity 1997, 78: 284-292.

109. Silverman HB: Social organization and behaviour of the narwhal, Monodon monoceros L. in Lancaster Sound, Pond Inlet and Tremblay Sound, Northwest Territories. M.Sc. Thesis, McGill University, Montreal, Quebec; 1979.

110. Reeves RR, Tracey S: Monodon monocerus. Mammalian Species 1980, 127:1-7.

111. Brodie PF: White whale, Delphinapterus leucas (Pallas 1776). In: Handbook of Marine Mammals Edited by Ridgway SH, Harrison Sir H. New York: Academic Press; 1989: 119-141. [vol.4].

112. Bel'kovitch VM, Kirillova OI: Investigation of white whales (Delphinapterus leucas) biology in reproductive period. Zoologichesky Zhurnal 2000, 79:89-96.

113. Litovka DI: Distribution of the Beluga Delphinapterus leucas in the Anadyr Estuary in 2000. Russian Journal of Marine Biology 2002, 28: 263-266.

114. Lønø O, Øynes P: White whale fishery at Spitzbergen. Norway Whaling Gaz 1961, 50: 267-286.

115. Lukin LR, Vasil'ev LY: Distribution of white whales (Delphinapterus leucas) in the White Sea and the Southeastern region of the Barents Sea during the ice season. Russian Journal of Marine Biology 2004, 35:236-241.

116. Lydersen C, Martin AR, Kovacs KM, Gjertz I: Summer and autumn movements of white whales Delphinapterus leucas in Svalbard, Norway. Marine Ecology Progress Series 2001, 219: 265-274.

117. Michaud R: Social organization of the St. Lawrence beluga whale. 13th Biennial Conference Biology Marine Mammals: 28 Nov-3 Dec 1999; Wailea, Maui, Hawaiii.

118. Richard PR, Martin AR, Orr JR: Summer and autumn movements of belugas of the Eastern Beaufort Sea stock. Arctic 2001, 54: 223-236. 
119. Smith TG, Hammill MO, Martin AR: Herd composition and behaviour of white whales (Delphinapterus leucas) in two Canadian arctic estuaries. Meddelelser om Grønland, Bioscience 1994, 39: 175-184.

120. Dawson SM: Cephalorhynchus dolphins. In: Encyclopedia of Marine Mammals Edited by Perrin WF, Wursig B, Thewissen JGM. San Diego: Academic Press; 2002: 200-204.

121. Lescrauwaet AC, Gibbons J, Guzman L, Schiavini A: Abundance estimation of Commerson's dolphin in the eastern area of the Strait of Magalla-Chile. Revista Chilena de Historia Natural 2000, 73: 473-478.

122. Goodall RNP: Chilean dolphins Cephalorhynchus eutropia (Gray, 1846). In: Handbook of Marine Mammals Edited by Ridgway SH, Harrison Sir H. New York: Academic Press; 1994: 269-287. [vol.5].

123. Bräger S: Association patterns in three populations of Hector's dolphin, Cephalorhynchus hectori. Canadian Journal of Zoology 1999, 77:13-18

124. Slooten E, Dawson SM: Hector's dolphin Cephalorhynchus hectori (van Beneden, 1881). In: Handbook of Marine Mammals Edited by Ridgway SH, Harrison Sir H. New York: Academic Press; 1994: 311-333. [vol.5].

125. Slooten E: Behaviour of Hectorís dolphins: classifying behaviour by sequence analysis. Journal of Mammalogy 1994, 75: 956-964.

126. Slooten E: Population Biology, Social Organisation and Behaviour of Hectorís Dolphin. PhD thesis. University of Canterbury, Christchurch; 1990.

127. Slooten E, Dawson SM, Whitehead H: Associations among photographically identified Hectorís dolphins. Canadian Journal of Zoology 1993, 71: 2311-2318.

128. Best PB, Abernethy RB: Heavyside's dolphin Cephalorhynchus heavisisdii (Gray, 1828). In: Handbook of Marine Mammals Edited by Ridgway SH, Harrison Sir H. New York: Academic Press; 1994:289-310. [vol.5].

129. Brownell RLJr, Crespo EA, Donahue MA: Lagenorhynchus australis (Peale, 1848). In: Handbook of Marine Mammals Edited by Ridgway $\mathrm{SH}$, Harrison Sir H. New York: Academic Press; 1999: 105-120. [vol.6].

130. Viddi FA, Lescrauwaet AK: Insights on habitat selection and behavioural patterns of Peale's dolphins (Lagenorhynchus australis) in the Strait of Magellan, Southern Chile. Aquatic Mammals 2005, 31:176-183. 
131. Goodall RN: Peale's Dolphin (Lagenorhynchus australis). In: Encyclopedia of Marine Mammals Edited by Perrin WF, Wursig B, Thewissen JGM. San Diego:

Academic Press; 2002: 890-894.

132. Goodall RN: Hourglass Dolphin (Lagenorhynchus cruciger). In: Encyclopedia of Marine Mammals Edited by Perrin WF, Wursig B, Thewissen JGM. San Diego:

Academic Press; 2002: 583-585.

133. Brownell RLJr, Donahue MA: Lagenorhynchus cruciger (Quoy and Gaimard, 1824). In: Handbook of Marine Mammals Edited by Ridgway $\mathrm{SH}$, Harrison Sir H. New York: Academic Press; 1999: 121-137. [vol.4].

134. Van Waerebeek K, Würsig B: Pacific white-sided dolphin and dusky dolphins. In: Encyclopedia of Marine Mammals Edited by Perrin WF, Wursig B, Thewissen JGM. San Diego: Academic Press; 2002: 859-861.

135. Morton A: Occurrence, photo-identification and prey of Pacific White-sided dolphins (Lagenorhynchus obliquidens) in the Broughton Archipelago, Canada 1984-1998. Marine Mammal Science 2000, 16:80-93.

136. Ferguson MC, Barlow J, Fiedler P, Reilly SB, Gerrodette T: Spatial models of delphinids (family Delphinidae) encouter rate and group size in the eastern tropical Pacific Ocean. Ecological Modelling 2006, 193:645-662.

137. Markowitz TM: Social organization of the New Zealand dusky dolphin. Ph.D. Thesis. Texas A\&M University, 2004. 255 pp.

138. Brownell, RLJr, Cipriano F: Lagenorhynchus obscurus (Gray, 1828). In:

Handbook of Marine Mammals Edited by Ridgway SH, Harrison Sir H. New York:

Academic Press; 1999: 85-104. [vol.6].

139. Würsig B, Würsig M: Behavior and ecology of the dusky dolphins,

Lagenorhynchus obscurus, in the South Atlantic. Fishery Bulletin 1980, 77: 871-890.

140. Würsig B, Bastida R: Long-range movement and individual association of two dusky dolphins (Lagenorhynchus obscurus) off Argentina. Journal of Mammalogy 1986, 67: 773-774.

141.Würsig B, Pearson H, Markowitz T: Delphinid Behavioral Flexibilities: Social Strategies in Dusky Dolphins. Kyoto Conference. Delphinid and Primate Social Ecology: A Comparative Discussion: July 29 - 30 2005; Kyoto, Japan. 
142. Cipriano F: Atlantic White-Sided Dolphin (Lagenorhynchus acutus). In:

Encyclopedia of Marine Mammals Edited by Perrin WF, Wursig B, Thewissen JGM. San Diego: Academic Press; 2002: 49-50.

143. Reeves RR, Smeenk C, Brownell RLJr, Kinze CC: Atlantic white-sided dolphinLagenorhynchus acutus (Gray 1828). In: Handbook of Marine Mammals Edited by Ridgway SH, Harrison Sir H. New York: Academic Press; 1999: 31-57. [vol. 6].

144. Sergeant DE, Aubin DJ, Gerarci JR: Life history and northwest Atlantic status of the Atlantic white-sided dolphin, Lagenorhynchus acutus. Cetology 1980, 37: 1-12

145. Leatherwood S, Walker WA: The northern right whale dolphin Lissodelphis borealis Peale in the eastern North Pacific. In Behavior of marine mammals Edited by Winn HE, Olla BL. New York: Plenum Press; 1979: 85-141. [vol.3].

146. Jefferson TA, Newcomer MW, Leatherwood S, Van Waebebeek K: Righ whale dolphins-Lissodelphis borealis (Peale 1848) and Lissodelphis peronni (Lacepede, 1804). In: Handbook of Marine Mammals Edited by Ridgway SH, Harrison Sir H. New York: Academic Press; 1994:335-363. [vol.5].

147. Jefferson TA, Newcomer MW: Lissodelphis borealis. Mammalian Species 1993, 425: $1-6$.

148. Cruickshank RA, Brown SG: Recent observations and some historical records of southern right-whale dolphins Lissodelphis peronii. Fisheries Bulletin (South Africa) 1981, 15: 109-121.

149. Newcomer MW, Jefferson TA, Brownell RLJr: Lissodelphis peronii. Mammalian Species 1996, 531: 1-5.

150. Van Waerebeek KJ, Canto J, Gonzalez J, Oporto J, Brito JL: Southern right whale dolphins, Lissodelphis peronii off the Pacific coast of South America. Zeitschrif für Säugetierkunde 1991, 56:284-295.

151. Perrin WF: Common Dolphins (Delphinus delphis). In: Encyclopedia of Marine Mammals Edited by Perrin WF, Wursig B, Thewissen JGM. San Diego: Academic Press; 2002: 245-248.

152. Bearzi G, Reeves RR, Notarbartolo-Di-Sciara G, Politi E, Cañadas A, Frantzis A, Mussi B: Ecology, status and conservation of short-beaked common dolphins Delphinus delphis in the Mediterranean Sea. Mammal Review 2003, 33:224-252.

153. Evans WE: Common dolphin, white-bellied porpoise Delphinus delphis Linnaeus, 1758. In: Handbook of Marine Mammals Edited by Ridgway SH, Harrison Sir H, vol. 5. New York: Academic Press; 1994:191-224. 
154. Bruno S: Il metodo della fotoidentificazione applicato allo studio della socioecologia di delfini comuni (Delphinus delphis) nel Mar Ionio orientale, Degree in Biological Sciences Thesis. University of Padova, Italy 2001.

155. Bruno S, Politi E, Bearzi G: Social organisation of a common dolphin community in the eastern Ionian Sea: evidence of a fluid fission-fusion society. European Research on Cetaceans 2004, 15.

156. Perrin WF, Hohn AA: Pantropical spotted dolphin Stenella attenuata (Gray 1846). In: Handbook of Marine Mammals Edited by Ridgway SH, Harrison Sir H. New York: Academic Press; 1994: 71-98. [vol. 5.]

157. Perrin WF: Stenella attenuata. Mammalian Species 2001, 683: 1-8.

158. Perrin WF: Pantropical spotted dolphins (Stenella attenuata). In: Encyclopedia of Marine Mammals Edited by Perrin WF, Wursig B, Thewissen JGM. San Diego:

Academic Press; 2002: 865-867.

159. Pryor K, Shallenberger IK: Social structure in spotted dolphins (Stenella attenuata) in the tuna purse seine fishery in the eastern tropical Pacific. In: $O n$ behavior: essays and research Edity by K. Pryor. Washington DC: Sunshine Books; 1995: 135-172.

160. May-Collado LJ, Morales-Ramirez A: Presencia y patrones de comportamiento del delfín manchado costero, Stenella attenuata graffmani (Cetacea: Delphinidae) en el Golfo de Papagayo, Costa Rica. Revista de Biología Tropical 2005, 53: 265-276

161. Kasuya $\mathrm{T}$ : Reconsidertion of life history parameters of the spotted and striped dolphins based on cemental layers. The Scientific Report of Whales Research Institute 1976, 28: 73-106.

162. Kasuya T: Overview of cetacean life histories: an essay in their evolution. In: Whales, seals, fish and man Edited by AS Blix, Walløe L, Ulltang Ø. Netherlands: Elsevier Science; 1995: 481-497.

163. Perrin WF, Mead JG: Clymene Dolphin, Stenella clymene (Gray, 1828). In: Handbook of Marine Mammals Edited by Ridgway SH, Harrison Sir H. New York: Academic Press; 1994: 161-172. [vol.5].

164. Mullin KD, Higgins LV, Jefferson TA, Hansen LJ: Sightings of the Clymene dolphin (Stenella clymene) in the Gulf of Mexico. Marine Mammal Science 1994, 10: 464-470.

165. Jefferson TA, Curry BE: Stenella clymene. Mammalian Species 2003, 726: 1-5. 
166. Jefferson TA: Clymene Dolphin (Stenella clymene). In: Encyclopedia of Marine Mammals Edited by Perrin WF, Wursig B, Thewissen JGM. San Diego: Academic Press; 2002: 234-236.

167. Jefferson TA, Odell DK, Prunier KT: Notes on the biology of the Clymene dolphin (Stenella clymene) in the northern Gulf of Mexico. Marine Mammal Science 1995, 11:564- 573.

168. Herzing DL: Vocalizations and associated underwater behavior of free-ranging Atlantic spotted dolphins, Stenella frontalis, and bottlenose dolphins, Tursiops truncatus. Aquatic Mammals 1996, 22: 61-79.

169. Herzing DL: The natural history of free-ranging Atlantic spotted dolphins (Stenella frontalis): age classes, color phases, and female reproduction. Marine Mammals Science 1997, 13: 40-59.

170. Herzing DL: Acoustics and social behavior of wild dolphins: implications for a sound society. In Hearing by whales and dolphins Edited by Au WWL, Popper AN, Fay RE. New York: Springer Press; 2000: 225-272.

171. Perrin WF: Atlantic spotted dolphin (Stenella frontalis). In: Encyclopedia of Marine Mammals Edited by Perrin WF, Wursig B, Thewissen JGM. San Diego: Academic Press; 2002: 47-49.

172. Perrin WF, Caldwell DK, Caldwell MC: Atlantic spotted dolphin Stenella frontalis (G. Cuvier, 1829). In: Handbook of Marine Mammals Edited by Ridgway SH, Harrison Sir H. New York: Academic Press; 1994: 173-190. [vol.5].

173. Perrin WF: Stenella frontalis. Mammalian Species 2002, 702: 1-6.

174. Archer FI, Perrin WF: Stenella coeruleoalba. Mammalian Species 1999, 603:1-9.

175. Perrin WF, Wilson CE, Archer FI: Striped dolphin. Stenella coeruleoalba (Meyen, 1833). In: Handbook of Marine Mammals Edited by Ridgway $\mathrm{SH}$, Harrison Sir H. New York: Academic Press; 1994:129-159.[vol.5].

176. Perrin WF: Spinner dolphin (Stenella longirostris). In: Encyclopedia of Marine Mammals Edited by Perrin WF, Wursig B, Thewissen JGM. San Diego: Academic Press; 2002: 1174-1178.

177. Perrin WF, Gilpatrick JW: Spinner dolphin, Stenella longirostris (Gray, 1828). In: Handbook of Marine Mammals Edited by Ridgway SH, Harrison Sir H. New York: Academic Press; 1994:99-128. [vol.5].

178. Perrin WF: Stenella longirostris. Mammalian Species 1998, 599: 1-7. 
179. Würsig B, Wells RS, Würsig M, Norris KS: Population structure. In: The Hawaiian spinner dolphin First Edition Edited by Norris KS, Würsig B, Wells RS, Würsig M. Berkeley: University of California Press; 1994: 122-140.

180. Norris KS, Wursig B, Wells RS, Würsig M: The Hawaiian Spinner dolphin. Berkeley: University of California Press; 1994.

181. Karczmarski L, Würsig B, Gailey G, Larson KW, Vanderlip C: Spinner dolphins in remote Hawaiian atoll: social grouping and population structure. Behavioral Ecology and Sociobiology 2005, 16:675-685.

182. Wells RS: The role of long-term study in understanding the social structure of a bottlenose dolphin community. In: Dolphin societies: discoveries and puzzles, First Edition Edited by Pryor K, Norris KS. Berkeley: University of California Press; 1991: 199-225.

183. Wells RS, Scott MD, Irvine AB: The social structure of free-ranging bottlenose dolphins. Current Mammalogy 1987, 1: 247-305

184. Wells RS: Dolphin social complexity: lessons from long-term study and life history. In: Animal social complexity: intelligence, culture, and individualized societies, First Edition Edited by Waal FBM, Tyack PL. Cambridge: Harvard University Press; 2003: 32-56.

185. Würsig B: Occurrence and group organization of Atlantic bottlenose porpoises (Tursiops truncatus) in an Argentine bay. Biological Bulletin 1978, 154: 348-359

186. Defran RH, Weller DW: The occurrence, distribution, and site fidelity of bottlenose dolphins (Tursiops truncatus) in San Diego, California. Marine Mammal Science 1999, 15: 366-380.

187. Defran RH, Weller DW, Kelly DL, Espinoza MA: Range characteristics of Pacific bottlenose dolphins within the Southern California Bight. Marine Mammal Science 1999, 15: 381-393.

188. Connor RC, Smolker RA, Richards AF: Dolphin alliances and coalitions. In: Coalitions and Alliances in Humans and Other Animals Edited by Harcourt AH, de Waal FBM. Oxford: Oxford University Press; 1992: 415-443.

189. Smolker RA, Richards AF, Connor RC, Pepper JW: Sex differences in patterns of association among Indian Ocean bottlenose dolphins. Behaviour 1992, 123: 38-69

190. Smolker R, Pepper JW: Whistle convergence among allied male bottlenose dolphins (Delphinidae, Tursiops spp.). Ethology 1999, 105: 595-617. 
191. Smolker R, Mann J, Smuts B: Use of signature whistles during separations and reunions between bottlenose dolphin mothers and infants. Behavioral Ecological Sociobiology 1993, 33: 393-402.

192. Samuels A, Gifford T: A quantitative assessment of dominance relations among bottlenose dolphins. Marine Mammal Science 1997, 13: 70-99.

193. Parsons KM, Durban JW, Claridge DE, Balcomb KC, Noble LS, Thompson PM: Kinship as a basis for alliance formation between male bottlenose dolphins, Tursiops truncatus, in the Bahamas. Animal Behaviour 2003, 66: 185-194.

194. Möller LM, Beheregaray LB, Harcourt RG, Krutzen M: Alliance membership and kinship in wild male bottlenose dolphins (Tursiops aduncus) of southeastern Australia. Proceedings of the Royal Society of London, Series B 2001, 268: 1941-1947.

195. Lusseau D, Schneider K, Boisseau OJ, Haase P, Slooten E, Dawson SM: The bottlenose dolphin community of Doubtful Sound features a large proportion of long-lasting associations. Can geographic isolation explain this unique trait? Behavioral Ecology Sociobiology 2003, 54: 396-405.

196. Connor RC, Whitehead H: Alliances II. Rates of encounter during resource utilization: a general model of intrasexual alliance formation in fission-fusion societies. Animal Behaviour 2005, 69: 127-132.

197. Gero S, Bejder H, Whitehead H, Mann J, Connor RC: Behaviourally specific preferred associations in bottlenose dolphins Tursiops spp. Canadian Journal of Zoology 2005, 83:1566-1573.

198. Rogers C: The social structure of bottlenose dolphins, Tursiops truncatus in the Bahamas. Marine Mammal Science 2006, 20:688-708.

199. Krützen M, Barré LM, Connor RC, Mann J, Sherwin WB: 'O father:where art thou?'- Paternity assessment in an open fission-fusion society of wild bottlenose dolphins (Tursiops sp.) in Shark Bay, Western Australia. Molecular Ecology 2004, 13:1975-1990.

200. Amano M, Miyazaki N, Yanagisawa F: Life history of Fraser's dolphin, Lagenodelphis hosei, based on a school captured off the Pacific coast of Japan. Marine Mammal Science 1996,12:199-214.

201. Dolar MLL: Fraser's Dolphin (Lagenodelphis hosei). In: Encyclopedia of Marine Mammals Edited by Perrin WF, Wursig B, Thewissen JGM. San Diego: Academic Press; 2002:485-486. 
202. Jefferson TA, Leatherwood S: Lagenodelphis hosei. Mammalian Species 1994, 470: $1-5$.

203. Leatherwood S, Jefferson TA, Norris JC, Stevens WE, Hansen LJ, Mullin KD: Occurrence and sounds of Fraser's dolphins (Lagenodelphis hosei) in the gulf of Mexico. The Texas Journal of Science 1993, 45: 349-354.

204. Perrin WF, Leatherwood S, Collet A: Fraser's dolphin-Lagenodelphis hosei (Fraser 1956). In: Handbook of Marine Mammals Edited by Ridgway SH, Harrison Sir H. New York: Academic Press; 1994: 225-241. [vol.5].

205. Saayman GS, Tayler CK: The socioecology of humpback dolphins (Sousa sp.). In: The behaviour of marine animals Edited by Winn HE, Olla BL. New York: Plenum Press; 1979:165-226. [vol.3].

206. Karczmarski L: Group dynamics of humpback dolphins (Sousa chinensis) in the Algoa Bay region, South Africa. Journal of Zoology London 1999, 249: 283-293.

207. Karczmarski L, Cockcroft VG, McLachlan A:Group size and seasonal pattern of occurrence of humpback dolphins Sousa chinensis in Algoa Bay, South Africa. South African Journal of Marine Science 1999, 21: 89-97.

208. Karczmarski L, Winter P, Cockcroft VG, McLachlan A: Population analyses of Indo-Pacific humpback dolphins Sousa chinensis in Algoa Bay, Eastern Cape, South Africa. Marine Mammal Science 1999, 15: 1115-1123.

209. Guissamulo AT, Cockcroft VG: Ecology and population estimates of the IndoPacific humpback dolphins (Sousa chinensis) in Maputo Bay, Mozambique. Aquatic Mammals 2004, 30: 94-102.

210. Jefferson TA, Karczmarski L: Sousa chinensis. Mammalian Species 2001, 655:1-9.

211. Da Silva VMF, Best RC: Tucuxi-Sotalia fluviatilis (Gervais 1853). In: Handbook of Marine Mammals Edited by Ridgway SH, Harrison Sir H. New York: Academic Press; 1994: 43-70. [vol.5].

212. Da Silva VMF, Best RC: Sotalia fluviatilis. Mammalian Species 1996, 527: 1-7.

213. Montero-Filho ELA: Group organization of the dolphin Sotalia fluviatilis guianensis in an estuary of southeastern Brazil. Ciencia e Cultura Journal of the Brazilian Association for the Advancement of Science 2000, 52: 97-101.

214. Flores PAC: Tucuxi Sotalia fluviatilis. In: Encyclopedia of Marine Mammals Edited by Perrin WF, Wursig B, Thewissen JGM. San Diego: Academic Press; 2002: 1267-1269. 
215. Azevedo AF, Viana SC, Oliveira AM, Van Sluys M: Group characteristics of marine tucuxis (Sotalia fluviatilis) (Cetacea: Delphinidae) in Guanabara Bay, southeastern Brazil. Journal of the Marine Biology Association of the United Kingdom 2005, 85:209-212.

216. Gamboa-Poveda M, May-Collado LJ: Insights on the occurrence, residency, and behavior of two coastal dolphins from Gandoca-Manzanillo, Costa Rica: Sotalia guianensis and Tursiops truncatus (Family Delphinidae). International Whaling Commission Scientific Committee Meeting Document 2006, SC/58/SM4: 1-9.

217. Acevedo-Gutiérrez A, DiBerardinis A, Larkin S., Larkin K., Forestell P: Social interactions between tucuxis and bottlenose dolphins in Gandoca-Manzanillo, Costa Rica. Latin American Journal of Aquatic Mammals 2005, 4:49-54.

218. Geise L, Gomes N, Cerqueira R: Behavior, habitat use and population size of Sotalia fluviatilis (Gervais, 1853) (Cetacea, Delphinidae) in the Cananeia Estuary Region, São Paulo, Brazil. Revista Brasileira de Biologia 1999, 59:183-194.

219. Mobley JR, Spitz SS, Forney KA, Grotefendt R, Forestell PH: Distribution and abundance of odontocete species in Hawaiian waters: preliminary results of 199398 aerial surveys. Southwest Fisheries Science Center Administrative Report 2000, LJ-00$14 \mathrm{C}$.

220. Miyasaki N, Perrin WF: Rough-toothed dolphin- Steno bredanensis (Lesson 1828). In: Handbook of Marine Mammals Edited by Ridgway $\mathrm{SH}$, Harrison Sir H. New York: Academic Press; 1994: 1-22. [vol.5].

221. Jefferson TA: Rough-Toothed Dolphin (Steno bredanensis). In: Encyclopedia of Marine Mammals Edited by Perrin WF, Wursig B, Thewissen JGM. San Diego:

Academic Press; 2002: 1055-1059.

222. Perkins JS, Miller W: Mass stranding of Steno bredanensis in Belize. Biotropica 1983, 15:235-236.

223. Ferrero RC, Hodder J, Cesarone J: Recent strandings of rough-toothed dolphins, Steno bredanensis, on the Oregon and Washington coasts. Marine Mammal Science 1994, 10:114-6.

224. Ross GJB, Leatherwood S: Pygmy killer whale Feresa attenuata Gray 1874. In: Handbook of Marine Mammals Edited by Ridgway SH, Harrison Sir H. New York: Academic Press; 1994: 487-404. [vol.5].

225. Donahue MA, Perryman WL: Pygmy Killer Whale (Feresa attenuata). In: Encyclopedia of Marine Mammals Edited by Perrin WF, Wursig B, Thewissen JGM. San Diego: Academic Press; 2002:1009-1010. 
226. Mignucci-Giannoni AA, Toyos-González GM, Pérez-Padilla J, Rodríguez-López MA, Overing J: Mass stranding of pygmy killer whales (Feresa attenuata) in the British Virgin Islands. Journal of the Marine Biology Association of the United Kingdom 2000, 80:759-760.

227. Williams AD, Williams $\mathrm{R}$, Brereton $\mathrm{T}$ : The sighting of pygmy killer whales (Feresa attenuata) in the southern Bay of Biscay and their association with cetacean calves. Journal of the Marine Biology Association of the United Kingdom 2002, 82:509511.

228. Amos B, Schlötterer C, Tautz D: Social structure of pilot whales revealed by analytical DNA profiling. Science 1993, 260: 670-672.

229. Bernard HJ, Reilly SB: Globicephala (Lesson 1828). In: Handbook of Marine Mammals Edited by Ridgway SH, Harrison Sir H. New York: Academic Press; 1999: 245-280. [vol.6.].

230. Norris KS. Prescott JH: Observations on Pacific cetaceans of California and Mexican waters. University of California Publications in Zoology 1961, 63: 291-402.

231. Kasuya, T. Japanese fisheries exploiting southern form short-finned pilot whales. International Whaling Commission Scientific Committee Meeting Document 1986. SC/38/SM19: 1-12.

232. Heimlich-Boran JR: Social organisation of the short-finned pilot whale, Globicephala macrorhynchus, with special reference to the comparative social ecology of Delphinids. PhD Thesis. Cambridge University; 1993.

233. Whitehead H, Rendell L, Osborne RW, Würsig B: Culture and conservation of non-humans with reference to whales and dolphins: review and new directions. Biological Conservation 2004, 120:427-437.

234 Connor RC: Group living in whales and dolphins. In: Cetacean Societies: Field studies of dolphins and whales. Edited by Mann J, Connor RC, Tyack PL, Whitehead H. Chicago: The University of Chicago Press; 2000: 199-218.

235. Ottensmeyer A, Whitehead H: Behavioural evidence for social units in long finned pilot whales. Canadian Journal of Zoology 2003, 81: 1327-1338.

236. Weilgart, LS, Whitehead H: Vocalizations of the North Atlantic pilot whale (Globicephala melas) as related to behavioural contexts. Behavioral Ecology and Sociobiology 1990, 26: 399-402. 
237. Fullard KJ, Early G, Heide-Jorgensen MP, Bloch D, Rosing-Asvid A, Amos W:

Population structure of long-finned pilot whales in the North Atlantic: a correlation with sea surface temperature? Molecular Ecology 2002, 9: 949-958.

238. Andersen LW, Siegismund HR: Genetic evidence for migration of males between schools of the long-finned pilot whale Globicephala melas. Marine Ecology Progress series 1994, 105:1-7.

239. Amano M, Miyazaki N: Composition of a school of Risso's dolphins, Grampus griseus. Marine Mammal Science 2004, 20:152-160.

240. Zucca P, Di Guardo G, Francese M, Scaravelli D, Genov T, Mazzatenta A: Causes of stranding in four Risso's dolphins (Grampus griseus) found beached along the North Adriatic Sea coast. Veterinary Research Communications 2005, 29: 261-264. 241. Baird RW: (2002a). Risso's Dolphin (Grampus griseus). In: Encyclopedia of Marine Mammals Edited by Perrin WF, Wursig B, Thewissen JGM. San Diego: Academic Press; 2002:1037-1039.

242. Kruse S: Aspects of the biology, ecology, and behavior of Risso's dolphis (Grampus griseus) off the California coast. M.Sc. Thesis. University of California at Santa Cruz; 1989.

243. Leatherwood S, Hubbs CL, Fisher M: First records of Risso's dolphin (Grampus griseus) from the Gulf of California with detailed notes on mass stranding. Transactions of the San Diego Society of Natural History 1979, 19: 45-52.

244. Jefferson TA, Barros NE: Peponocephala electra. Mammalian Species 1997, 553: $1-6$.

245. Miyasaki N, Yoshihiro F, Iwata K: Biological analysis of a mass stranding of melon-headed whales (Peponochela electra) at Aoshima, Japan. Bulletin of the National Science Museum Series A Tokyo 1998, 24: 31-60.

246. Perryman WL: Melon-headed whale (Peponocephala electra). In: Encyclopedia of Marine Mammals Edited by Perrin WF, Wursig B, Thewissen JGM. San Diego: Academic Press; 2002:733-734.

247. Stayce PJ, Leatherwood S, Baird RW: Pseudorca crassidens. Mammalian Species 1994, 456: 1-6.

248. Odell DK, McClune KM: False Killer Whale-Pseudorca crassidens. In: Handbook of Marine Mammals Edited by Ridgway SH, Harrison Sir H. New York: Academic Press; 1999: 213-244. [vol.6]. 
249. Baird RW: False Killer Whale (Pseudorca crassidens). In: Encyclopedia of Marine Mammals Edited by Perrin WF, Wursig B, Thewissen JGM. San Diego: Academic Press; 2002:411-412.

250. Amano M: Odontoceti social structure and life history parameters: interspecific comparisons. Kyoto Conference, Delphinid and Primate Social Ecology: a comparative discussion. Kyoto, Japan, July 2005: 29-30.

251. Arnold PW: Irrawaddy dolphin (Orcaella brevirostris). In: Encyclopedia of Marine Mammals Edited by Perrin WF, Wursig B, Thewissen JGM. San Diego: Academic Press; 2002: 652-655.

252. Marsh H, Lloze R, Heinsohn GE, Kasuya T. Irrawady Dolphin, Orcaella brevirostris (Gray 1866). In: Handbook of Marine Mammals Edited by Ridgway SH, Harrison Sir H. New York: Academic Press; 1989:101-118. [vol.4].

253. Parra GJ, Corkeron PJ, Marsh H: Population sizes, site fidelity and residence patterns of Australian snubfin and Indo-Pacific humpback dolphins: Implications for conservation. Biological Conservation 2006, 129: 167-180

254. Stacey PJ, Arnold PW: Orcaella brevirostris. Mammalian Species 1999, 616:1-8.

255. Bigg MA, Olesiuk PF, Ellis GM, Ford JKB, Balcomb KC: Social organization and genealogy of resident killer whales (Orcinus orca) in the coastal waters of British Columbia and Washington State. Report of the International Whaling Commission, Special Issue 1990, 12: 383-405.

256. Baird RW, Abrams PL, Dill LM: Possible indirect interactions between transient and resident killer whales: implications for the evolution of foraging specializations in the genus Orcinus. Oecologia 1992, 89: 125-132.

257. Baird RW, Dill LM: Ecological and social determinants of groups size in transient killer whales. Behavioral Ecology 1996, 7: 408-16.

258. Baird RW, Whitehead H: Social organization of mammal-eating killer whales: group stability and dispersal patterns. Canadian Journal of Zoology 2000, 78: 20962105.

259. Baird RW: The killer whale: foraging specializations and group hunting. In: Cetacean Societies: Field Studies of Dolphins and Whales Edited by Mann J, Connor RC, Tyack PL, Whitehead H. Chicago: University of Chicago Press; 2000:127-154.

260. Yurk H, Barrett-Lennard LG, Ford JKB, Matkin CO: Cultural transmission within maternal lineages: Vocal clans in resident killer whales in Southern Alaska. Animal Behaviour 2002, 63: 1103-1119. 
261. Ford JKB: Vocal traditions among resident killer whales (Orcinus orca) in coastal waters of British Columbia. Canadian Journal of Zoology 1991, 69: 14541483.

262. Heyning JE, Dahlheim ME: Orcinus orca. Mammalian Species 1988, 304:1-9.

263. Brault S, Caswell H: Pod-specific demography of killer whales (Orcinus orca). Ecology 1993, 74:1444-1454.

264. Hoezel AR, Dahlheim M, Stern SJ: Low genetic variation among whales (Orcinus orca) in the Eastern North Pacific and genetic differentiation between foraging specialists. Journal of Heredity 1998, 89:121-128.

265. Hoezel AR, Natoli A, Dahlheim ME, Olavarria C, Baird RW, Black NA: Low worldwide genetic diversity in the killer whale (Orcinus orca): implications for demographic history. Proceeding of the Royal Society of London, Series B 2002, 269:1467-1473.

266. Guinet C: Intentional stranding apprenticeship and social play in killer whales (Orcinus orca). Canadian Journal of Zoology 1991, 69:2712-2716. 
267. Parks SE, Tyack PL: Sound production by North Atlantic right whales (Eubalaena glacialis) in surface active groups. Journal of the Acoustical Society of America 2005,117: 3297-3306.

268. Ljungblad DK, Thompson PO, Moore SE: Underwater sounds recorded from migrating bowhead whales, Baleana mysticetus, in 1979. Journal of the Acoustical Society of America 1982, 71:477.

269. Clark CW, Johnson JH: The sounds of the bowhead whale, Balaena mysticetus, during the spring migrations of 1979 and 1980. Canadian Journal of Zoology 1984, 62:1436-1441.

270. Knowlton AR, Clark CW, Kraus SD: Sounds recorded in the presence of sei whales (B. borealis). $9^{\text {th }}$ Biennial Conference on the Biology of Marine Mammals: $5^{\text {th }}-9^{\text {th }}$ December 1991; Chicago. 1991:40.

271. McDonald MA, Hildebrand JA, Wiggins SM, Thiele D, Glasgow D, Moore SE: Sei whale sounds recorded in the Antarctic. Journal of the Acoustical Society of America 2005, 118:3941-3945.

272. Cummings WC, Thompson PO, Ha SJ: Sounds from Bryde's, Balaenoptera edeni, finback, B. physalus, whales in the Gulf of California. Fish Bulletin U. S. 1986, 84:359-370.

273. Edds P: Vocalisations of a captive juvenile and free-raning adult-calf pairs of Bryde's whales, Balaenoptera edeni. Marine Mammal Science 1993, 9: 269-284.

274. Heimlich SL, Mellinger DK, Nieukirk SL, Fox CG: Types, distribution, and seasonal occurrence of sounds attributed to Bryde's whales (Balaenoptera edeni) recorded in the eastern tropical Pacific, 1999-2001. Journal of the Acoustical Society of America 2005, 118:1830-1837.

275. Mellinger DK, Clark CW: Blue whale (Balaenoptera musculus) sounds from the North Atlanctic. Journal of the Acoustic Society of America 2003, 114:1108-1119.

276. Stafford KM, Nicukirk, SL, Fox CG: Geographic and seasonal variation of blue whale calls in the North Pacific. Journal of Cetacean Research and Management 2001, 3:65-76.

277. Alling A. Personal Communication to Mellinger DK and Clark CW. In Mellinger DK, Clark CW: Blue whale (Balaenoptera musculus) sounds from the North Atlanctic. Journal of the Acoustic Society of America 2003, 114:1108-1119. 
278. Ljungblad DK, Stafford KM, Shimada H: Sound attributed to blue whales recorded off the southwest coast of Australia in December 1995. Reports of the International Whaling Commission 1997, 47: 435-439.

279. Watkins WA, Daher MA, George JE, Rodriguez D: Twelve years of tracking 52Hz whale calls from a unique source in the North Pacific. Deep-Sea Research I 2004, 51:1889-1901.

280. Sirovic A, Hildebrand JA, Wiggins SM, McDonald MA, Moore SE, Thiele D: Seasonality of blue and fin whales calls and the influence of sea ice in the Western Antarctic Peninsula. Deep-Sea Research II 2004, 51:2327-2344.

281. Stafford KM: Acoustic detection and location of blue whales (Balaenoptera musculus) from SOSUS by matched filtering. Journal of the Acoustical Society of America 1994, 96:3250.

282. Berchok CL, Bradley DL, Gabrielson TB: St. Lawrence blue whale vocalizations revisited: Characterization of calls detected from 1998-2001. Journal of the Acoustical Society of America 2006, 120:2340-2354.

283. Edds P: Characteristics of finback, Balaenoptera physalus, vocalizations in the St. Lawrence Estuary. Bioacoustics 1988, 1:131-149.

284. Watkins WA: The 20 Hz signals of finback whales (Balaenoptera physalus). Journal of the Acoustical Society of America 1987, 82:1901-1912.

285. Thompson PO, Friedl WA: A long term study of low frequency sounds from several species of whales off Oahu, Hawaii. Cetology 1982, 45: 1-19.

286. Hafner GW, Hamilton CL, Steiner WW, Thompson TJ, Winn HE: Signature information in the song of the humpback whale. Journal of the Acoustical Society of America 1979, 66:1-6.

287. Cummings WC, Thompson PO, Cook R: Underwater sounds of migrating gray whales, Eschrichtius glaucus. Journal of the Acoustical Society of America 1968, 44:1278-1281.

288. Fish JF, Sumich JL, Lingle GL: Sounds produced by the gray whale, Eschrichtius robustus. Marine Fisheries Review 1974, 36:38-45.

289. Dahlheim ME, Fisher HD, Schempp JD: Sound production by the gray whale and ambient noise levels in Laguna San Ignacion, Baja California Sur, Mexico. In: The Gray Whale Edited by Jones ML, Swartz L, Leatherwood S. Orlando: Academic Press; 1984: 511-541. 
290. Dawbin WH, Cato DH: Sounds of a pygmy right whale (Caperea marginata). Marine Mammal Science 1992, 8:213:219.

291. May-Collado LJ, Wartzok D: The freshwater dolphin Inia geoffrensis geoffrensis produces high frequency whistles. Journal of Acoustical Society of America 2007, 121: in press

292. Wang D, Wursig B, Evans WE: Comparisons of whistles among seven odontocete species. In: Sensory Systems of Aquatic Mammals Edited by Kastelein RA, Thomas JA, and Nachtigal PE. The Netherlands: DeSpil; 1995: 299-323

293. Wang D, Wursig B, Leatherwwod S: Whistles of boto, Inia geoffrensis, and tucuxi, Sotalia fluviatilis. Journal of the Acoustical Society of America 2001, 109: 407414.

294. Diazgranados MC, Trujillo F: Vocal repertoire of the freshwater dolphins Inia geoffrensis and Sotalia fluviatilis in Colombia, South America. Journal of the Acoustical Society of America 2002, 112: 2400.

295. Wang D, Wang K, Akamatsu T, Fujita F: Study on whistles of the Chinese River Dolphin or baiji Lipotes vexillifer. Oceanologia et Limonologia Sinica 1999, 30:349354.

296. Wang X, Wang D, Akamatsu T, Fujita K, Shiraki R: Estimated detection distance of a baiji's (Chinese river dolphin, Lipotes vexillifer) whistles using a passive acoustic survey method. Journal of the Acoustical Society of America 2006, 120: 13611365.

297. Ford JKB, Fisher HD: Underwater acoustic signals of the narwhal (Monodon monocerus). Canadian Journal of Zoology 1978, 56: 552-560.

298. Watkins WA, Schevill WE, Ray C: Underwater sounds of Monodon (Narwhal). Journal of the Acoustical Society of America 1970, 49: 595-599.

299. Shapiro A: Preliminary evidence for signature vocalizations among free-ranging narwhals (Monodon monocerus). Journal of the Acoustical Society of America 2006, 120: $1695-1705$.

300. Karlsen JD, Bisther A, Lydersen C, Haug T, Kovacs KM: Summer vocalisations of adult male white whales (Delphinapterus leucas) in Svalbard, Norway. Polar Biology 2002, 25:808-817.

301. Sjare BL, Smith TG: The vocal repertoire of white whales, Delphinapterus leucas, summering in Cunningham Inlet, Northwest Territories. Canadian Journal of Zoology 1986, 64: 407-415. 
302. Belikov RA, Bel'kovich VM: Characteristics of white sea beluga whale (Delphinapterus leucas Pall) whistle-like signals. XI Session of the Russian Acoustical Society, November 2001; Moscow, 2001: 19-23.

303. Caldwell MC, Caldwell DK: Statistical evidence for individual signature whistles in the Pacific whitesided dolphin, Lagenorhynchus obliquidens. Cetology 1970, 16:121.

304. Yin SE: Movement patterns, behaviors, and whistle sounds of dolphin groups off Kaikoura, New Zealand. M.Sc. Thesis. Texas A\&M University; 1999: 107 pp.

305. Steiner WW: Species-specific differences in pure tonal whistle vocalizations of five western North Atlantic dolphin species. Behavioral Ecology Sociobiology 1981, 9:241-246.

306. Moore SE, Ridgway SH: Whistles produced by common dolphins from Southern California Bight. Aquatic Mammals 1995, 21:55-63.

307. Oswald JN, Barlow J, Norris TF: Acoustic identification of nine delphinids species in the eastern tropical Pacific Ocean. Marine Mammal Science 2003, 19: 2037.

308. Mullin KD, Higgins LV, Jefferson TA, Hansen LJ: Sightings of the Clymene dolphin (Stenella clymene) in the Gulf of Mexico. Marine Mammal Science 1994, 10 :464-470.

309. Watkins WA, Wartzok D: Sensory biophysics of marine mammals. Marine Mammal Science 1985, 1: 219-260.

310. Matthews JN, Rendell LE, Gordon JCD, MacDonald DW: A review of frequency and time parameters of cetacean tonal calls. Bioacoustics 1999, 10: 47-71. 21

311. Barzúa-Durán MC, Au WWL: Whistles of Hawaiian spinner dolphins. Journal of the Acoustical Society of America 2002, 112: 3064-3072.

312. Barzúa-Durán MC, Au WWL: Geographic variations in the whistles of spinner dolphins (Stenella longirostris) of the Main Hawaiian Islands. Journal of the Acoustical Society of America 2004, 116: 3757-3769.

313. Driscoll AD: The whistles of Hawaiian spinner dolphins, Stenella longirostris, M.Sc. Thesis, University of California at Santa Cruz; 1995.

314. Boisseau O: Quantifying the acoustic repertoire of a population: the vocalizations of free-ranging bottlenose dolphins in Fiordland, New Zealand. Journal of the Acoustical Society of America 2005, 117: 2318-2329. 
315. Wang D, Wursig B, Evans WE: Whistles of bottlenose dolphins: comparisons among populations. Aquatic Mammals 1995, 21: 65-77.

316. WatkinsWA, Daher MA, Fristrup KM, Notarbartolo di Sciara G: Fishing and acoustic behavior of Fraser's dolphin (Lagenodelphis hosei) near Dominica, southeast Caribbean. Caribbean Journal of Science 1994, 30:76-82.

317. Oswald JN, Rankin S, Barlow J: First description of whistles of Pacific Fraser's dolphins, Lagenodelphis hosei. Bioacoustics, submitted.

318. Leatherwood S, Jefferson TA, Norris JC, Stevens WE, Hansen LJ, Mullin KD: Occurrence and sounds of Fraser's dolphins (Lagenodelphis hosei) in the gulf of Mexico. The Texas Journal of Science 1993, 45:349-354.

319. Van Parijs SM, Corkeron PJ: Vocalizations and Behavior of Pacific Humpback dolphins Sousa chinensis. Ethology 2001, 107:701-716.

320. Zbinden K, Pilleri G, Kraus C, Bernath O: Observations on the behaviour and underwater sounds of the plumbeous dolphin (Sousa chinensis G. Cuvier 1829) in the Indus Delta region. In: Investigations on Cetacea Edited by Pilleri G. Germany: University of Bern; 1977: 259-286

321. Schultz KW, Corkeron PJ: Interspecific differences in whistles produced by inshore dolphins in Moreton Bay, Queensland, Australia. Canadian Journal of Zoology 1994, 72: 1061-1068

322. Azevedo AF, Van Sluys M: Whistles of tucuxi dolphins (Sotalia fluviatilis) in Brazil: comparisons among populations. Journal of the Acoustical Society of America 2005, 117: 1456-1464.

323. Azevedo AF, Simão SM: Whistles produced by marine tucuxi dolphins Sotalia fluviatilis in Guanabara Bay, southeastern Brazil. Aquatic Mammals 2002, 28: 261266.

324. Erber C, Simão SM: Analysis of whistles produced by the tucuxi dolphin Sotalia fluviatilis from Sepetiba Bay, Brazil. Annals of the Brazilian Academy of Sciences 2004, 76:381-385.

325. Podos J, da Silva VMF, Rossi-Santos MR: Vocalizations of Amazon river dolphins, Inia geoffrensis: insights into the Evolutionary origins of delphinid whistles. Ethology 2002, 108: 601-612.

326. Busnell RG, Dziedzic A: Caracteristiques physiques de certains signaux acoustiques du delphidide Steno bredanensis, Lesson. Comptes reduns del'Académie des Sciences Paris Series D 1968, 262:143-146. 
327. Rendell LE, Matthews JN, Gill A, Gordon JCD, MacDonald DW: Quantitative analysis of tonal calls from five odontocete species, examining interspecific and intraspecific variation. Journal of Zoology 1999, 249: 403-410.

328. Corkeron PJ, Van Parijs SM: Vocalizations of eastern Australian Risso's dolphins, Grampus griseus. Canadian Journal of Zoology 2001, 79: 160-164.

329. Watkins WA, Daher MA, Samuels A, Gannon DP: Observations of Peponocephala electra, the Melon-headed whale, in the southeastern Caribbean. Caribbean Journal of Science 1997, 33:34-40.

330. Van Parijs SM, Parra GJ, Corkeron PJ: Sounds produced by Australian Irrawaddy dolphins, Orcaella brevirostris. Journal of the Acoustical Society of America 2000, 108:1938-1940

331. Ford JKB: Acoustic behavior of resident killer whales (Orcinus orca) off Vancouver Island, Bristish Columbia (Canada). Canadian Journal of Zoology 1989, 67:727-745.

332. Dahlheim ME, Awbrey F: A classification and comparison of vocalizations of captive killer whales (Orcinus orca). Journal of the Acoustical Society of America 1982, 72:661-670.

333. Steiner WW, Hain JH, Winn HE, Perkins PJ: Vocalizations and feeding behavior of the killer whale (Orcinus orca). Journal of Mammalogy 1979, 60:823-827.

334. Thomsen F, Franck D, Ford JKB: Characteristics of whistles from the acoustic repertoire of resident killer whales (Orcinus orca) off Vancouver Island, British Columbia. Journal of the Acoustical Society of America 2001, 109:1240-1246.

335. Riesch R, Ford JKB, Thomsen F: Stability and group specificity of stereotyped whistles in resident killer whales, Orcinus orca, off British Columbia. Animal Behaviour 2006, 71:79-91 
VITA

LAURA JOHANNA MAY-COLLADO

May $6^{\text {th }} 1971$

1991-1995

1992-1996

1997-1998

2001-2002

$1998-2001$

1998-2003

2003-2007

2003-2005
Born, Puntarenas, Costa Rica

B.S. Biology

University of Costa Rica

San Jose, Costa Rica

Teaching Assistant/Museum of Zoology

Curator Assistant

(Mammalogy/Ornithology) University of Costa Rica

San Jose, Costa Rica

Visitor Scholar

Portland State University

Portland, USA

Teaching Assistant

Organization for Tropical Studies

San Jose, Costa Rica

M. Sc. Biology

University of Costa Rica

San Jose, Costa Rica

Research Assistant

Centro de Investigaciones del Mar y

Limnologia, University of Costa Rica,

San Jose, Costa Rica

Doctoral Candidate in Biology, Dept. of

Biological Sciences, Florida International

University

Miami, Florida, USA

Head Teaching Assistant

Teaching Assistant, Dept. of Biological

Sciences, Florida International University Miami, Florida, USA 


\section{PUBLICATIONS}

Agnarsson, I., Coddington, J.A., and L.J. May-Collado. In press. Elongated pedicillate setae- a putative sensory system and synapomorphy of spiders. Journal of Arachnology. May-Collado, L. J., Agnarsson, I., and D. Wartzok. 2007. Phylogenetic review of tonal sound production in whales in relation to sociality. Evolutionary Biology. 7(136).

May-Collado, L. J., Agnarsson, I., and D. Wartzok. 2007. Reexamining the relationship between body size and tonal signals frequency in whales: a phylogenetic comparative approach. Marine Mammal Science. Marine Mammal Science. 23 (3): 524-552.

May-Collado, L. J. and D. Wartzok. 2007. Inia geoffrensis high frequency whistles. Journal of the Acoustical Society of America. 121 (2): 1203-1212.

May-Collado, L. J. and I. Agnarsson. 2006. Cytochrome b and Bayesian inference of whale phylogeny. Molecular Phylogenetics and Evolution. 38: 344-354.

Agnarsson, I., Barrantes, G. and L. J. May-Collado. 2006. Notes on the biology of Anelosimus pacificus Levi, 1963 (Theridiidae, Araneae)-evidence for an evolutionary reversal to a less social state. Journal of Natural History. 40(47-48): 2681-2687.

Gamboa-Poveda M. and L. J. May-Collado. 2006 Insights on the occurrence, residency, and behavior of two coastal dolphins from Gandoca-Manzanillo, Costa Rica: Sotalia guianensis and Tursiops truncatus (Family Delphinidae). International Whaling Commission. SC/58/SM4.

May-Collado, L. J.; Gerrodete, T.; Calambokidis, J.; Rassumen K. and I. Sereg. 2005. Distribution of Cetaceans sightings in the EEZ of Costa Rica. Revista de Biología Tropical. Vol. 53 (1-2): 249-263.

May-Collado, L. J. and Morales Ramírez, A. 2005. Presencia y Patrones de comportamiento del delfín manchado costero, Stenella attenuata graffmani (Cetacea: Delphinidae) en el Golfo de Papagayo, Costa Rica. Revista de Biología Tropical. Vol. 53 (1-2): 265-276.

Rodríguez-Hernández, B. Chinchilla, F. A. and L. J. May-Collado. 2002. Lista de especies, endemismo, y conservación de los mamíferos de Costa Rica. Revista Mexicana de Mastozoología. 6: 19-41.

\section{PRESENTATIONS}

May-Collado, L. J. and M. Gamboa-Poveda (2006). Insights on the biology of Sotalia guianensis at Gandoca-Manzanillo, Costa Rica: residency, habitat use, acoustics, and reactions to anthropogenic noise. Abstract presented to the Sotalia Workshop, Rio de Janeiro, Brazil June 2006.

May-Collado, L.J. and D. Wartzok. (2005). The evolution of cetacean whistles: a phylogenetic comparative approach. Oral presentation at the16th Biennial Conference on the Biology of Marine Mammals, Dec 12-16. San Diego. 